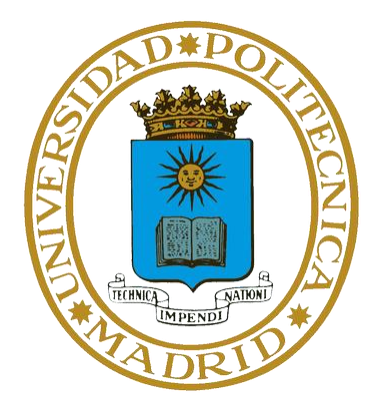

DEPARTAMENTO DE BIOTECNOLOGÍA-BIOLOGÍA VEGETAL ESCUELA TÉCNICA SUPERIOR DE INGENIERÍA AGRONÓMICA, ALIMENTARIA Y DE BIOSISTEMAS

UNIVERSIDAD POLITÉCNICA DE MADRID

\title{
ECOLOGICAL DETERMINANTS OF POTYVIRUS EMERGENCE IN WILD ECOSYSTEMS
}

\section{DOCTORAL THESIS}

\section{CRISTINA RODRÍGUEZ NEVADO}

Master's Degree in Genetics and Cell Biology 

DEPARTAMENTO DE BIOTECNOLOGÍA-BIOLOGÍA VEGETAL ESCUELA TÉCNICA SUPERIOR DE INGENIERÍA AGRONÓMICA, ALIMENTARIA Y DE BIOSISTEMAS

UNIVERSIDAD POLITÉCNICA DE MADRID

\section{ECOLOGICAL DETERMINANTS OF POTYVIRUS EMERGENCE IN WILD ECOSYSTEMS}

\section{DOCTORAL THESIS}

\section{CRISTINA RODRÍGUEZ NEVADO}

Master's Degree in Genetics and Cell Biology

Supervisor:

\section{JESÚS ISRAEL PAGÁN MUÑOZ}

Universidad Politécnica de Madrid PhD

Madrid, 2018 



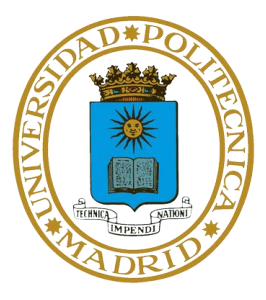

UNIVERESIDAD POLITÉCNICA DE MADRID

Tribunal nombrado por el Magfco. y Excmo. Sr. Rector de la Universidad Politécnica de Madrid, el día de de 2018

Presidente: $\mathrm{D} / \mathrm{D}^{\mathrm{a}}$

Secretario: D/Da

Vocal: $\mathrm{D} / \mathrm{D}^{\mathrm{a}}$

Vocal: $\mathrm{D} / \mathrm{D}^{\mathrm{a}}$

Vocal: D/D

Suplente: D/Da

Suplente: $\mathrm{D} / \mathrm{D}^{\mathrm{a}}$

Realizado el acto de defensa y lectura de Tesis el día de de 2018 En el Centro de Biotecnología y Genómica de Plantas, UPM-INIA.

EL PRESIDENTE LOS VOCALES

EL SECRETARIO 



\section{AGRADECIMIENTOS}

Esta tesis ha sido realizada en el grupo Determinantes de Transmisión y Especiación de los Virus de Plantas dirigido por el Dr. Jesús Israel Pagán Muñoz perteneciente al Centro de Biotecnología y Genómica de Plantas UPMINIA, (CBGP-UPM-INIA). Me gustaría expresar mi agradecimiento a las siguientes personas por su contribución en la elaboración del presente trabajo:

Dr. Jesús Israel Pagán Muñoz por la dirección de este trabajo, por la búsqueda de las localizaciones en los ecosistemas estudiados y por su ayuda en los muestreos de campo. Además, quiero destacar su importante labor en mi formación científica y su valiosa dedicación a la planificación y supervisión de esta tesis.

Dr. Fernando García-Arenal Rodríguez, por ampliar mi formación en virología y por haber facilitado los medios necesarios para la elaboración de este trabajo dentro de su grupo Interacción Planta-Virus y Co-evolución.

Dra. Rosario G. Gavilán García (Universidad Complutense de Madrid, UCM) por la identificación de las plantas muestreadas en los ecosistemas de este estudio y por sus recomendaciones para realizar la caracterización ecológica de los mismos.

Dr. Edward Holmes (The University of Sydney, Australia) por sus enseñanzas sobre los métodos filodinámicos y filogenéticos empleados en este trabajo, así como por concederme la oportunidad y los medios necesarios para desarrollar parte del mismo durante mi estancia en la Universidad de Sídney.

Dras. Beatriz García Jiménez (Centro Nacional de Biotecnología, CNB) por su colaboración en el desarrollo de los análisis de Aprendizaje Automático y por sus explicaciones sobre la elaboración de los mismos y Nuria Montes Casado (Instituto de Investigación Sanitaria Hospital Universitario de La Princesa, Madrid) por su ayuda y colaboración en el desarrollo de los análisis de estadística multivariante.

A Viji Vijayan por su asistencia en la purificación del virus MeRV, y a Marina García de Lomana, Alberto Cobos y Maria Luisa López Herranz por su excelente ayuda técnica sin la cual este trabajo no habría sido posible. 
Por último, quiero reconocer a la UPM por el apoyo económico recibido gracias a las "Ayudas para estancias breves en España y en el extranjero para los beneficiarios de los programas predoctorales oficiales de formación de investigadores", según la Resolución del 26 de mayo de 2015 (Convocatoria 2015).

Este trabajo ha sido financiado por una beca Marie Curie de Integración Profesional ("Marie Curie Career Integration Grant", PCIG11-GA-2012-322100) (Años 2013-2016). 
Quisiera agradecer a Israel Pagán la oportunidad de formar parte de su grupo. Gracias por su inestimable ayuda, paciencia y la confianza depositada en mí para la realización de este trabajo. Gracias también por su valiosa orientación científica y personal durante estos años.

Quiero agradecer también su ayuda y apoyo a todas las personas que forman 0 formaron parte del laboratorio. En especial a Nuria Montes y Livia Donaire por su acogida y atención desde que llegué, siempre dispuestas a ayudarme y transmitirme sus conocimientos. Os he echado de menos. A mi compañera en el grupo, Viji Vijayan por todo lo que hemos compartido juntas durante esta experiencia. A Miguel Ángel Mora y Antolín López por sus ocurrencias, que nos hacen pasar ratos tan divertidos. También quiero agradecer a las Dras. Aurora Fraile, Soledad Sacristán y María Ángeles Ayllón por sus útiles consejos y contribuciones en las dudas científicas durante este trabajo. Quisiera dar las gracias también a Marisa López por su siempre eficiente ayuda técnica en los últimos meses. A todos, mi más sincero agradecimiento.

Gracias a todos los estudiantes que me han ayudado en los muestreos de campo y en el laboratorio por su dedicación, entusiasmo y simpatía, en especial a Alberto y Marina, por los buenos ratos en el campo y las interesantes conversaciones entre extracto y extracto que me animaban el día.

También quisiera agradecer su ayuda a todos los compañeros de las antípodas. Todos ellos hicieron que, a pesar de la gran distancia, me sintiera como en casa y que mi estancia fuera una experiencia magnífica.

Gracias a mis amigos por su comprensión y buenos consejos. En especial, gracias a David, por todo su apoyo y ánimos en los momentos de incertidumbre. Gracias por las sonrisas, la energía positiva y la fuerza que me contagias cada día.

Y, sobre todo, gracias a mi familia. A mi abuela, que me pudo acompañar hasta el principio de esta etapa, por su infinito cariño, y a mis padres, por su afecto y la ayuda que siempre he recibido de ellos antes y después de volar del nido. Por vuestro apoyo incondicional, muchísimas gracias. 



\section{INDEX}

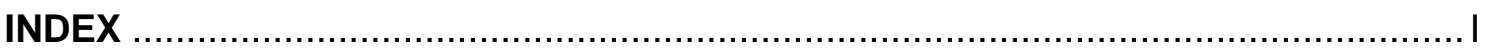

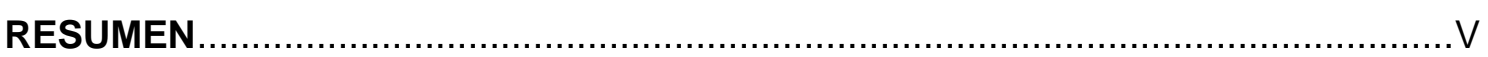

ABSTRACT

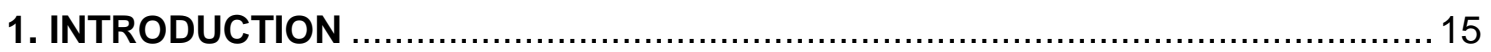

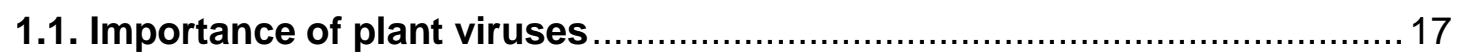

1.2. Ecological factors driving emergence of plant viruses ............................ 19

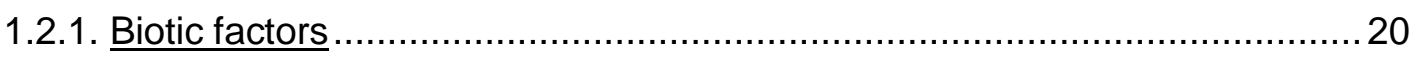

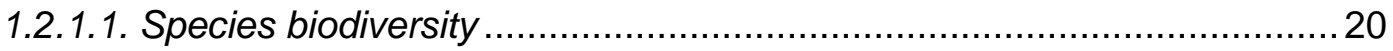

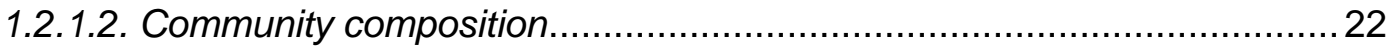

1.2.1.3. Host density and biomass .......................................................... 23

1.2.1.4. Pathogen host range............................................................... 24

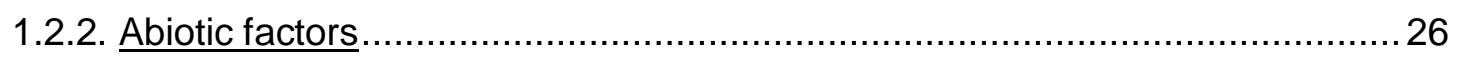

1.2.2.1. Effect of abiotic factors on host physiology .......................................... 26

1.2.2.2. Effect of abiotic factors on the vector population ..................................2 27

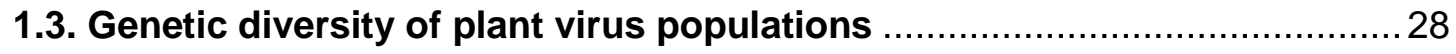

1.3.1. Generation of genetic diversity in plant virus populations...........................28

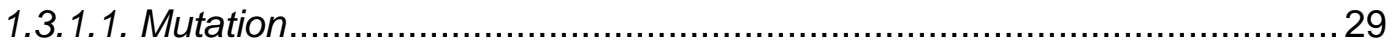

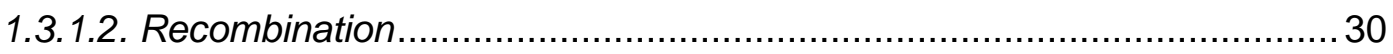

1.3.1.3. Processes that Shape the Genetic Diversity of Plant Virus Populations 30

1.3.2. Ecological factors affecting virus population genetic diversity ..................... 32

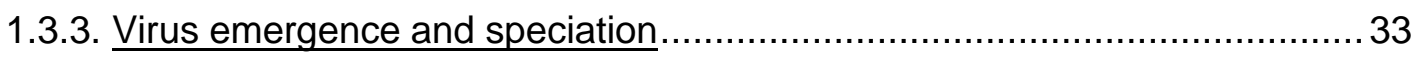

1.4. Wild ecosystems in the Iberian Peninsula ................................................. 34

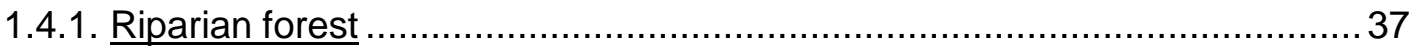

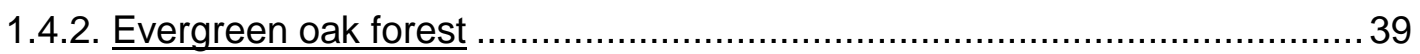

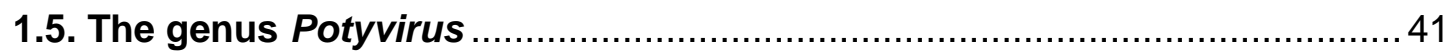

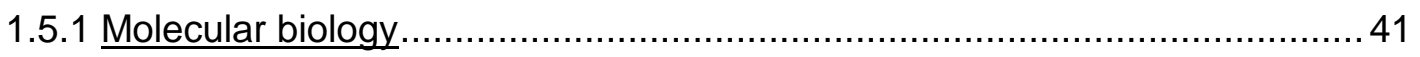

1.5.2. Ecology, epidemiology and disease control .......................................... 44

1.5.3. Genetic diversity and evolution .................................................... 47

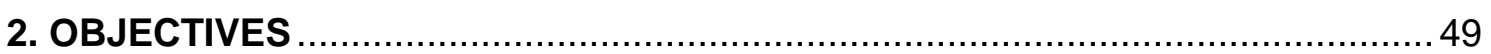

3. MATERIALS AND METHODS

3.1. Plant sampling and data collection in wild ecosystems ...........................55

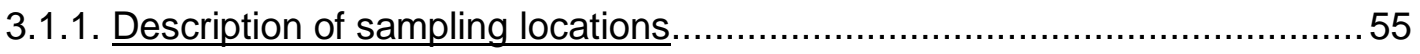

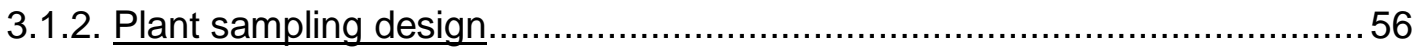


3.1.3. Monitoring and estimation of ecological factors......................................5 57

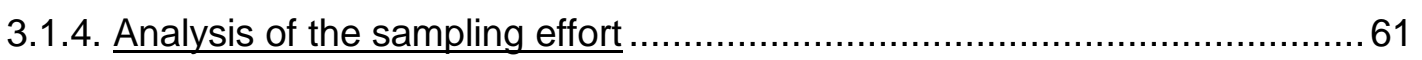

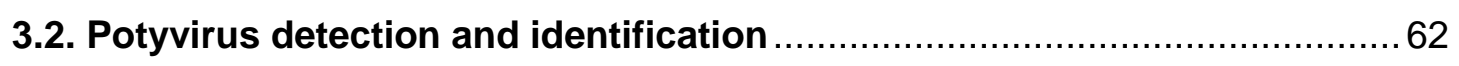

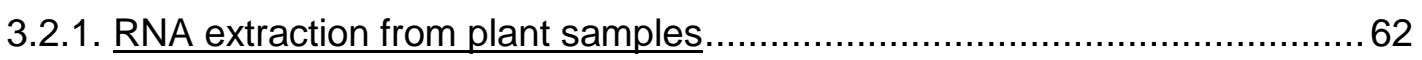

3.2.2. Detection and identification of virus species of the genus Potyvirus............. 63

3.2.3. Phylogenetic characterization of potyvirus species .................................6. 64

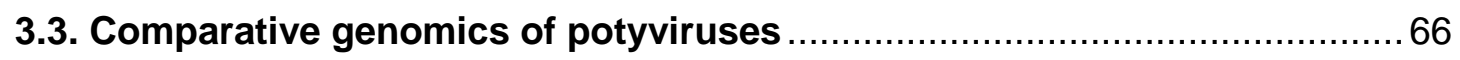

3.3.1. Detection of recombination in potyvirus populations ...............................66

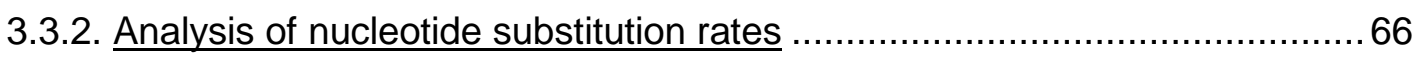

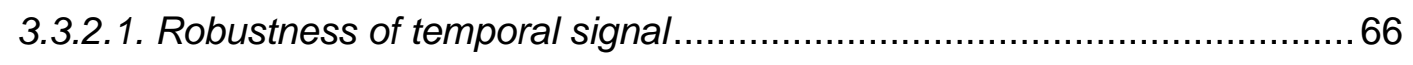

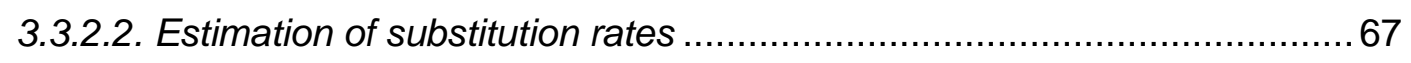

3.3.3. Analysis of potyvirus population genetic diversity .................................67

3.3.4. Analysis of the genetic structure of potyvirus populations ...........................68

3.4. Biological and molecular characterization of a novel potyvirus in a wild

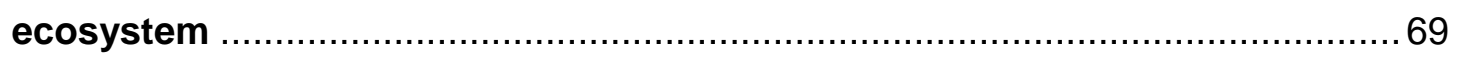

3.4.1. Biological characterization of Mediterranean ruda virus (MeRV) .................69

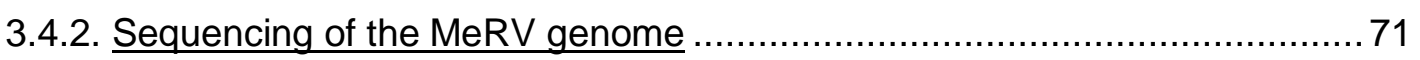

3.4.3. Phylogenetic analyses of ParP17-MeRV ............................................ 73

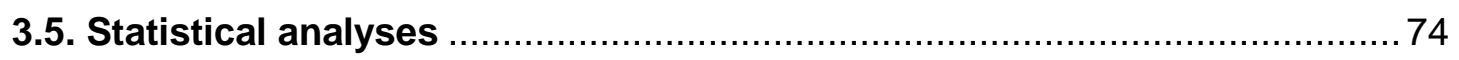

3.5.1. Comparison of floristic composition and potyvirus prevalence between

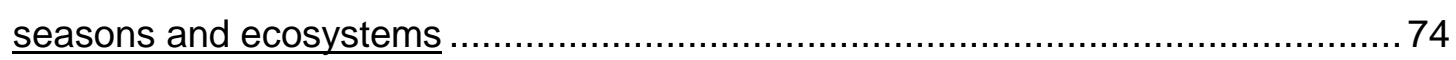

3.5.2. Analysis of ecological factors affecting infection risk and genetic diversity of

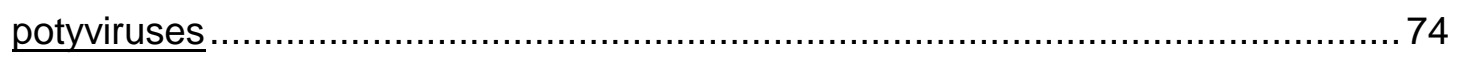

3.5.2.1. Analysis at potyvirus genus-wide level............................................ 74

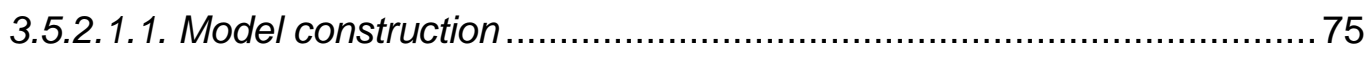

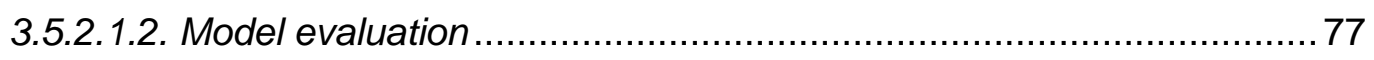

3.5.2.2. Analysis at potyvirus species-specific level ...................................... 79

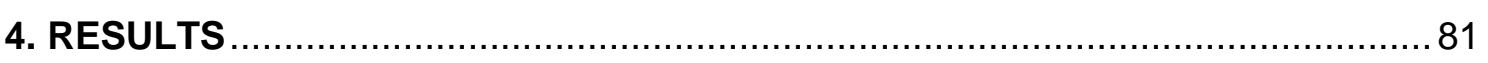

4.1. Characterization of evergreen oak forest and riparian forests in the lberian

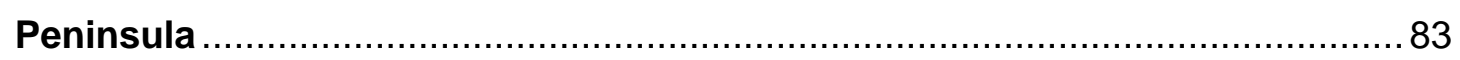

4.1.1. Analysis of plant biodiversity and floristic composition ............................... 84

4.1.2. Analysis of variation in abiotic factors between riparian and evergreen oak

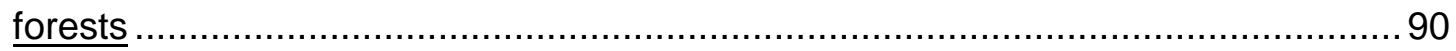

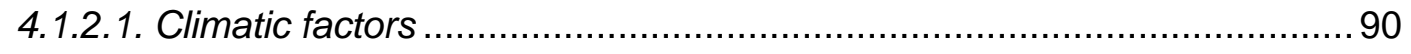

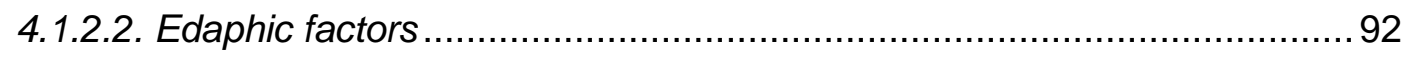

4.2. Analysis of genus-wide potyvirus prevalence in wild ecosystems of the Iberian Peninsula...................................................................................... 94 
4.2.1. Potyvirus prevalence and host abundance in evergreen oak and riparian

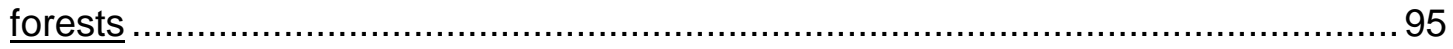

4.2.2. Ecological factors affecting Potyvirus genus-wide prevalence .................... 98

4.2.2.1. Complete ML models of genus-wide potyvirus prevalence.................. 101

4.2.2.2. ML models of genus-wide potyvirus prevalence excluding plant identity....

4.2.2.3. Role of $M L$ predictors in determining genus-wide potyvirus prevalence 111

4.2.2.3.1. ML models of genus-wide potyvirus presence/absence. 111

4.2.2.3.2. ML models of genus-wide potyvirus prevalence level.

4.3. Analysis of species-specific potyvirus prevalence in wild ecosystems in

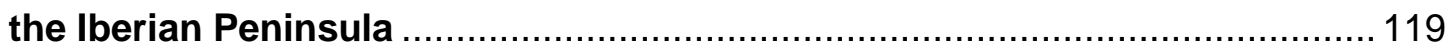

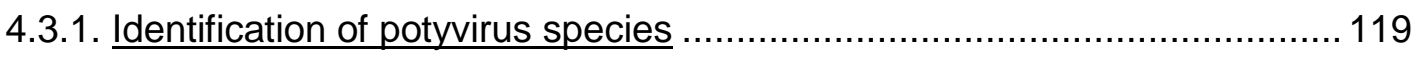

4.3.2. Potyvirus species-specific host range and prevalence ............................. 123

4.3.3. Ecological factors affecting MeRV prevalence ...................................... 125

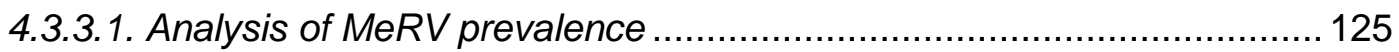

4.3.3.2. Analysis of ecological factors affecting MeRV prevalence ................... 127

4.3.4. Ecological factors affecting WHMV prevalence ...................................... 128

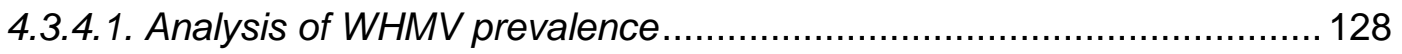

4.3.4.2. Analysis of ecological factors affecting WHMV prevalence .................. 131

4.4. Analysis of potyvirus genus-wide genetic diversity in wild ecosystems in the Iberian Peninsula .......................................................................... 133

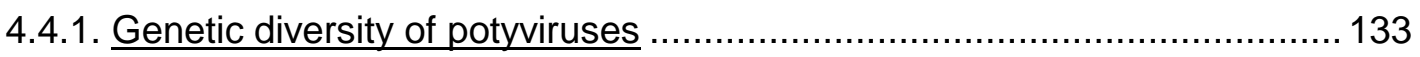

4.4.2. Analysis of recombination and nucleotide substitution rates of potyvirus populations.

4.4.3. Analysis of ecological factors affecting the genus-wide potyvirus population

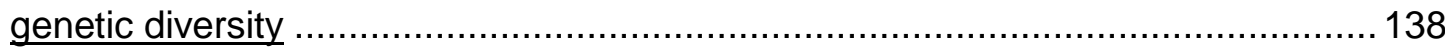

4.4.4. Analysis of potyvirus speciation modes............................................... 141

4.5. Analysis of potyvirus species-specific genetic diversity in wild ecosystems in the Iberian Peninsula.....

4.5.1. Analysis of the population genetic diversity of a specialist potyvirus in wild

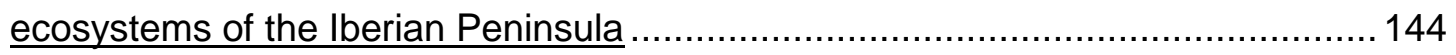

4.5.1.1. Analysis of the complete nucleotide sequence of MeRV ..................... 144

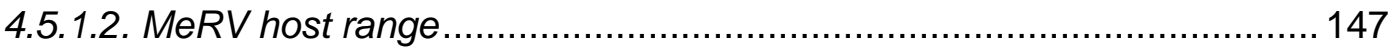

4.5.1.3. Analysis of MeRV population genetic diversity ................................. 148

4.5.1.4. Analysis of ecological factors affecting MeRV population genetic diversity. 
4.5.2. Analysis of the population genetic diversity of a generalist potyvirus in wild

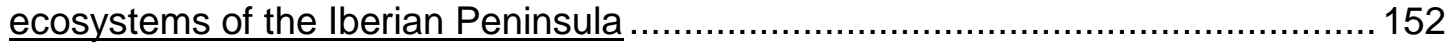

4.5.2.1. Analysis of WHMV population genetic diversity............................... 152

4.5.2.2. Analysis of ecological factors affecting WHMV population genetic diversity

4.5.2.3. Analysis of WHMV speciation mode ................................................. 156

5. DISCUSSION 159

5.1. Characterization of evergreen oak forest and riparian forests in the lberian Peninsula 161

5.2. Ecological determinants of genus-wide potyvirus infection risk in wild ecosystems

5.3. Ecological determinants of species-specific potyvirus infection risk in wild ecosystems.. 168

5.4. Potyvirus genus-wide population genetic diversity in wild ecosystems 173 5.5. Potyvirus species-specific population genetic diversity in wild ecosystems. .175

5.6. Limitations of the study 177

5.7. Concluding remarks 178

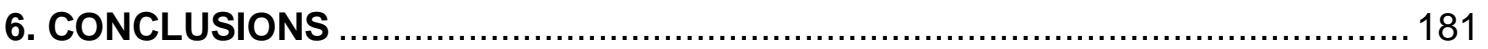

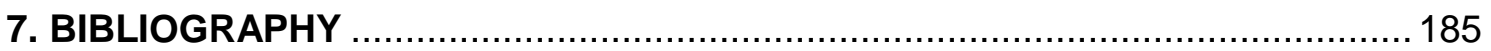

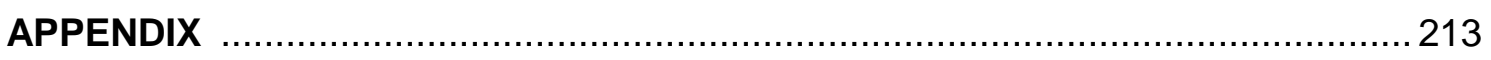

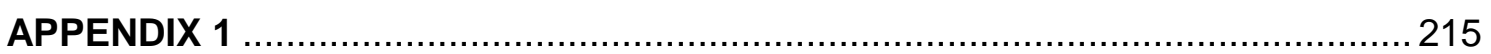

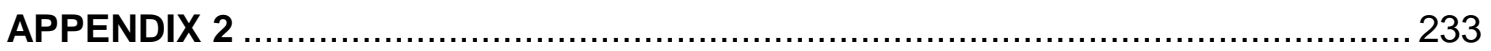

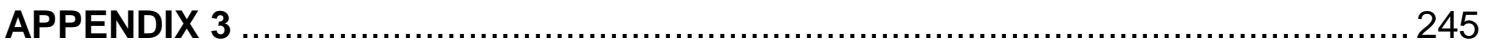

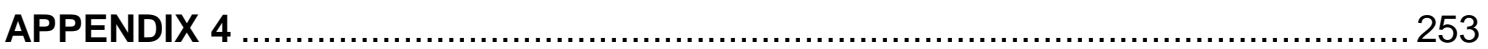

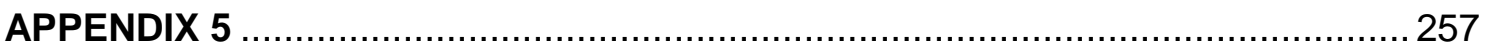

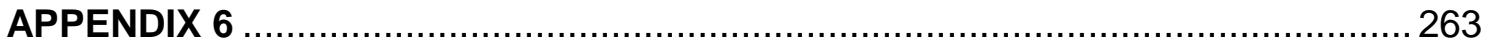


RESUMEN 
Cada vez son más numerosas las evidencias que indican que existe una amplia diversidad de virus de plantas en los ecosistemas silvestres. Sin embargo, la mayoría de las especies de virus presentes en estos ecosistemas permanecen sin caracterizar. Es más, apenas existen datos sobre los factores que afectan al riesgo de infección y a la diversidad genética de las poblaciones de virus que infectan huéspedes silvestres, ambos relacionados con los procesos de emergencia de virus. Esta tesis analiza los factores ecológicos que determinan el riesgo de infección y la diversidad genética de las poblaciones de virus de plantas en los ecosistemas silvestres. En concreto, la tesis se centra en las especies de virus del género Potyvirus presentes en los dos ecosistemas silvestres más representativos del centro de la Península lbérica: los bosques de ribera y los encinares. Estos análisis se basan en una caracterización detallada de los dos ecosistemas silvestres estudiados y de los potyvirus que se encuentran en ellos a lo largo de tres años: en localizaciones de ambos ecosistemas silvestres se ha analizado la prevalencia de potyvirus (como medida del riesgo de infección) y se han registrado las variaciones estacionales de diversos factores ecológicos y climáticos que caracterizan cada ecosistema. Los factores ecológicos que afectan a la prevalencia y a la diversidad genética poblacional de los potyvirus se identificaron a diferentes escalas taxonómicas (género vs. especie) y se consideró el efecto de la estrategia de vida del virus (especialista vs. generalista).

La comparación de los datos obtenidos en los dos ecosistemas estudiados indicó que la prevalencia de potyvirus fue generalmente más alta en los bosques de ribera, lo que se asoció con una mayor riqueza de especies de plantas y una mayor abundancia de plantas huéspedes, la mayoría de las cuales eran especies perennes. Este resultado está de acuerdo con las teorías que predicen que la identidad de las especies presentes en un ecosistema, y no solo su número, es un factor determinante del riesgo de infección por patógenos. Otros análisis presentados en esta tesis apoyan este resultado. Por ejemplo, cuando se estudió la asociación entre los factores ecológicos/climáticos y la prevalencia de potyvirus dentro de cada ecosistema se identificaron la composición de la comunidad vegetal, y la abundancia relativa y la densidad del/los huésped(es) como los factores más importantes que afectan a la prevalencia de potyvirus en los bosques de ribera y en los 
encinares. Un análisis más preciso del papel de estos factores ecológicos en la prevalencia/riesgo de infección de potyvirus reveló que cada uno está involucrado en una etapa diferente de la emergencia viral: la composición de la comunidad vegetal determinaría el riesgo de que los potyvirus entren en el ecosistema. Una vez dentro del ecosistema, la abundancia relativa/densidad del huésped determinaría la dispersión de los potyvirus, probablemente a través de su efecto en la eficacia de transmisión de los virus. Por lo tanto, determinaría el grado de infección (prevalencia) en las poblaciones de huéspedes. Curiosamente, la gama de huéspedes de cada especie de potyvirus afecta a la importancia relativa de cada uno de estos factores ecológicos: la densidad del huésped es el principal predictor de la prevalencia de un virus especialista, mientras que la abundancia relativa del huésped lo es de la prevalencia de un virus generalista.

Los cambios en la epidemiología de los patógenos pueden dar como resultado modificaciones en la estructura genética en sus poblaciones. Por lo tanto, cambios en la prevalencia de los potyvirus pueden ir acompañados de variaciones en la diversidad genética de sus poblaciones. De hecho, el análisis de los factores ecológicos que afectan a la diversidad genética de las poblaciones de potyvirus en bosques de ribera y encinares indicaron que la abundancia relativa y la densidad del/los huésped(es) fueron factores determinantes de dicha diversidad genética. Además, junto con estos factores ecológicos, la prevalencia de los potyvirus también fue un predictor importante de la diversidad genética de las poblaciones virales. Los resultados de esta tesis revelan además que la importancia relativa de estos predictores depende de la gama de huéspedes de cada especie de virus: la abundancia relativa de los huéspedes fue el factor más determinante de la diversidad genética de las poblaciones de un virus generalista, mientras que la prevalencia fue el principal predictor de la diversidad genética en las poblaciones de un virus especialista. Los resultados de esta tesis también indican que, en los dos ecosistemas silvestres, otros factores ecológicos y climáticos, tal vez vinculados a la dinámica de las poblaciones de los vectores de los potyvirus, juegan un papel importante en la evolución de estos virus. Finalmente, esta tesis ha investigado por primera vez los patrones y procesos de especiación dentro del género Potyvirus en ecosistemas silvestres. Los resultados apoyan que el proceso de 
diversificación genética de los potyvirus es compatible con eventos de especiación simpátrica en los ecosistemas estudiados.

En resumen, los resultados de esta tesis ofrecen información relevante sobre los factores determinantes de la emergencia de virus de plantas en ecosistemas silvestres y destacan la importancia de considerar la interacción entre la ecología, la epidemiología y la diversidad genética de las poblaciones de huéspedes y virus de plantas con el fin de comprender los procesos que conducen a la emergencia de virus de plantas en ecosistemas silvestres. 
ABSTRACT 
Increasing evidence indicates that viruses are widespread and highly diverse in wild ecosystems. Most of the virus species present in wild ecosystems however, remain uncharacterized. Moreover, in these ecosystems the factors affecting plant virus infection risk and population genetic diversity, two traits linked to virus emergence, are largely unknown. This thesis analyses the ecological determinants of plant virus infection risk and population genetic diversity in wild ecosystems. The thesis focuses on virus species of the genus Potyvirus present in the two most representative wild ecosystems in the centre of the Iberian Peninsula: riparian and evergreen oak forests. These analyses are based on a detailed characterization of these wild ecosystems and of the potyviruses that populate them: along three years, potyvirus prevalence (as a measure if infection risk), and seasonal data about ecological and climatic factors, were recorded in locations of both wild ecosystems. The ecological determinants affecting the prevalence and population genetic diversity of potyviruses were identified at different taxonomical scales (genus vs. species) and considering different virus life-history strategies (specialist $v s$. generalist).

Comparative analyses of potyvirus prevalence between ecosystems indicated that virus prevalence was generally higher in riparian forests, which was associated with higher plant species richness and abundance of host plants, most of which were perennial species. This result is in agreement with theories predicting that the identity of the species present in an ecosystem, and not only the number of species, is a key factor in determining pathogen infection risk. Further analysis reported in this thesis supports this result. For instance, analyses of the association between the ecological/climatic factors and the potyvirus prevalence within each ecosystem identified the plant community composition, the host relative abundance and the host density as the most important determinants of potyvirus prevalence in the two studied ecosystems. A fine study of the role of these ecological factors in potyvirus prevalence/infection risk revealed that they are involved in different stages of virus emergence: the plant community composition would determine the risk of potyvirus jump into the ecosystem. Once introduced in the ecosystem, host relative abundance/density would affect potyvirus invasion, likely affecting plantto-plant transmission, and thus determining the extent of the infection (prevalence) in the host populations. Interestingly, the host range of each 
potyvirus species appears to determine the relative importance of each of these ecological factors: host density was the chief predictor of the prevalence of a specialist virus, and host relative abundance was the major determinant of the prevalence of a generalist virus.

Epidemiological changes may result in genetic modifications in the pathogen population. Therefore, changes in potyvirus prevalence can be accompanied by variations in the virus population genetic diversity. Indeed, analyses of ecological factors affecting the genetic diversity of potyvirus populations in evergreen oak and riparian forests indicated that host relative abundance and density were also important determinants of such viral genetic diversification. Together with these ecological traits, prevalence was also a major predictor of virus population genetic diversity. As for potyvirus epidemiology, the results of this thesis reveal that the relative importance of these predictors depend on the virus species-specific host range: the population genetic diversity of a generalist virus was mainly determined by host relative abundance, whereas prevalence was the chief predictor of the population genetic diversity of a specialist virus. The results of this thesis also indicate that other ecological and climatic factors, perhaps linked to the dynamics of the virus vector populations, play an important role in the evolution of potyvirus populations in wild ecosystems. Finally, this thesis has investigated for the first time, the patterns and processes of speciation within the genus Potyvirus in wild ecosystems, demonstrating that the process of genetic diversification of potyviruses is compatible with sympatric speciation events in the studied ecosystems.

In summary, the results of this thesis provide relevant information on the determinants of plant virus emergence in wild ecosystems and highlight the importance of considering the interplay between the ecology, epidemiology and genetic diversity of plant host and virus populations in order to fully understand the processes that lead to plant virus emergence in wild ecosystems. 
1. INTRODUCTION 


\subsection{Importance of plant viruses}

Plant diseases are one of the major constraints on agricultural production worldwide, with a socioeconomic impact that can be comparable to those caused by human and animal diseases. Around $16 \%$ of global crop yields are reduced each year due to plant pathogens, which pose a serious risk to food security (Oerke, 2006; Savary et al., 2006). Although losses caused by plant viruses alone are difficult to estimate, plant viruses are considered the second most damaging crop pathogens only after fungi/oomycetes (Hsu, 2002). Indeed, according to some estimates plant viruses cause $7 \%$ of yield reductions in crops yearly (Oerke and Dehne, 2004). However, the economic impact of individual viral epidemics can be enormous. For instance, strains of Cassava mosaic begomoviruses cause 25 million tons of cassava losses every year in Africa, India and Sri Lanka; Citrus tristeza virus (CTV) is responsible for losses in citrus tree cultures estimated to over 100 million trees worldwide; and viruses affecting rice cultures result in yield losses estimated in more than US $\$ 1.5$ billion in South-East Asia alone (reviewed by Nicaise, 2014). The threat posed by plant virus diseases increases under the current conditions of climate change and global trade as these conditions favour the emergence of new diseases. Emerging infectious diseases, including those caused by plant viruses, can be defined as infections that have appeared in a new host population or have previously existed in a host population but are rapidly increasing in incidence or geographic range due to changes in its epidemiology (Woolhouse, 2002; 2005). The negative impact of diseases is greatest in the case of emerging ones, since they affect host populations that have not been previously challenged by the causal agent (Vurro et al., 2010; Woolhouse et al., 2005). Viruses account for half of the reported emerging infectious diseases from plants reported in the last four decades (Anderson et al., 2004). For instance, in the past 15 years Pepino mosaic virus (PepMV) (Hanssen and Thomma, 2010; Gómez et al., 2012) and Tomato yellow leaf curl virus (TYLCV) (Moriones and Navas-Castillo, 2000; Hanssen et al., 2010) have emerged in tomato crops causing devastating epidemics worldwide. 
Besides being important crop pathogens, viruses are present in wild ecosystems (Cooper and Jones, 2006; Roossinck, 2010; Prendeville et al., 2012), in which they are highly diverse and widespread (Roossinck, 2012; Stobbe and Roossinck, 2014), and they may be important ecological agents. For instance, quantitative resistance of wild plants to viruses have been described (Gilbert, 2002; Pagán et al., 2010; Moreno-Pérez et al., 2014), suggesting that these may affect the host population composition. Also, viral infection can drastically reduce the number of individuals in the host populations by decreasing the competitive and/or the reproductive abilities of infected plants, shaping host population size (Anderson et al., 2004; Malmstrom et al., 2005b; Vijayan et al., 2017) and, in some extreme cases, leading to local extinction of wild host populations (Futayama-Noguchi, 2001; Futayama et al., 2001). Viruses of wild ecosystems may also be important for agroecosystems (Figure 1.1). The intensification of agriculture has caused that an increasing proportion of wild plant communities have been reduced to patches that lie now surrounded by agricultural lands (Stukenbrock and McDonald, 2008; Alexander et al., 2014). The border between wild ecosystems and agricultural ecosystems represents an agro-ecological interface where crop species are in closer proximity to wild species. Plant viruses may move across this interface and induce the emergence of new infectious diseases (Alexander et al., 2014; Roossinck and García-Arenal, 2015; Malmstrom and Alexander, 2016). In this context, plant viruses infecting wild hosts may be important causal agents of emerging diseases in crops due to their ubiquity, dispersal capacity by vectors and ability to colonize both crop and wild plant species (Pagán et al., 2012; Alexander et al., 2014). Indeed, virus incidence in wild plants has been traditionally studied because of the role of these plants as the reservoirs of crop pathogens (Pinel-Galzi et al., 2015).

Most of the current knowledge on plant-virus interactions comes from the study of viruses that cause diseases in crops. Despite the importance of plant viruses for the conservation of wild ecosystems and for the emergence of infectious diseases in crops, little is known on their epidemiology and evolution and on the associated ecological determinants (Pagán et al., 2016). The study of the ecological factors and the evolutionary processes that determine the epidemiology and genetic structure of virus populations in wild ecosystems may 
contribute to develop more efficient strategies for controlling disease emergence, and to better understand the ecological role of viral infections in wild plant populations.

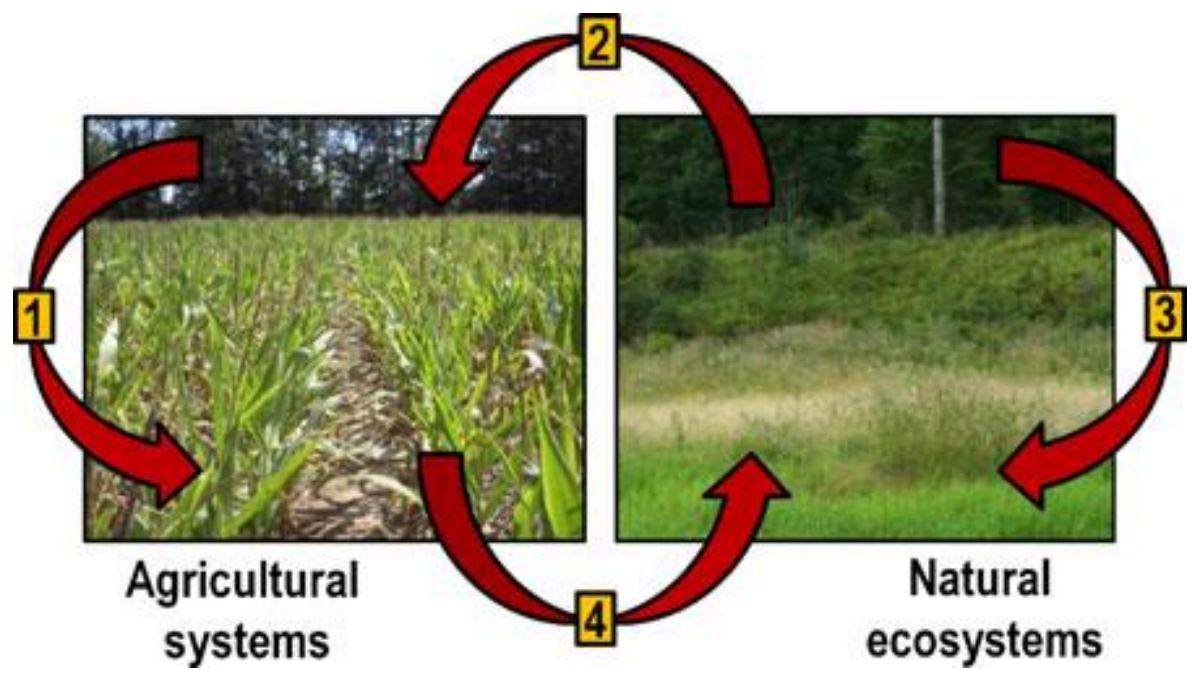

Figure 1.1. Virus interactions and virus movement at the agro-ecological interface. Numbers represent the relative amount known about each movement pathway $(1=$ most, $4=$ least). Figure taken from Alexander et al., 2014.

\subsection{Ecological factors driving emergence of plant viruses}

As for pathogens at large, virus emergence may be achieved through different processes, including spatial-spread to new environments, cross-species transmission, and increasing prevalence in a population where the pathogen is already present (Hudson et al., 2008; Holmes, 2009). The process of pathogen (including viruses) emergence through spatial-spread and cross-species transmission is complex and comprises multiple phases: In a first phase, preexisting genetic variants from host populations in which the pathogen is well established (reservoir hosts) are transmitted into a new habitat or new host species (spillover). In a second phase, the pathogen must adapt to the new host or habitat establishing productive infections through a process of adaptation. Finally, the pathogen must develop effective between-host transmission mechanisms in the new host/habitat to establish sustainable transmission cycles (Hudson et al., 2008; Holmes, 2009). Emergence can also occur in a host population in which the pathogen previously existed when the prevalence 
of the pathogen rapidly increases due to changes in the underlying epidemiology.

Emergence generally occurs in the context of complex ecological systems, and involves interactions between pathogens, hosts and vectors. Thus, ecological factors determining the population dynamics of these three interactive partners may affect the process of emergence (Jones, 2009; Elena et al., 2014). Changes in the ecology of hosts, pathogens and vectors may favor increasing contact between host populations, and hence pathogen host jumps or spillovers, and/or increase pathogen between-host transmission and incidence. Indeed, such ecological changes have been associated with the emergence of plant pathogens in both crop and wild plant species (Anderson et al., 2004; Power and Mitchell, 2004; Woolhouse et al., 2005). Also, ecological factors can enhance pathogen prevalence eventually leading to emergence. Some examples of factors that cause increased pathogen prevalence in plants are the introduction of new, more efficient vector species (Anderson et al., 2004; Fargette et al., 2006; Morales, 2006) and changes in the plant host community that enhance the abundance of hosts that maximize pathogen replication and/or vector reproduction (Borer et al., 2009; Pagán et al., 2012; Rodelo-Urrego et al., 2013). In the last three decades, the increasing number of emerging pathogens has boosted the interest of researchers for understanding the ecological factors affecting infection/disease risk (i.e. the ability of pathogens to infect their host/cause disease) that may finally lead to emergence of infectious diseases (Anderson et al., 2004; Keesing et al., 2006). This has resulted in the development of a significant body of theoretical work aiming at predicting the conditions that increase/decrease infection/disease risk (Ostfeld and Keesing, 2012; Johnson et al., 2015). According to these works, ecological factors that are potential determinants of infection/disease risk can be largely grouped into biotic and abiotic factors.

\subsubsection{Biotic factors}

\subsubsection{Species biodiversity}

Changes in the host ecology have been identified among the most frequent causes of infection/disease risk (Morse and Schluederberg, 1990; Jones, 2009). 
Because infectious diseases involve interactions between at least two species (host and pathogen), it has been proposed for a long time that ecosystem biodiversity will play a key role in infection risk (Burdon and Chilvers, 1982). Biodiversity is defined as the number and relative abundance of species and genotypes present in a given ecosystem (Wilson, 1992). Because different host species/genotypes are expected to vary in their susceptibility and ability to replicate or transmit specific pathogens, host diversity could play a central role in infection risk (Keesing et al., 2010; Ostfeld and Keesing, 2012). Two contrasting hypotheses relate ecosystem species diversity (i.e. the number of species or species richness) and host abundance to disease risk. The "Amplification Effect" hypothesis predicts that species diversity will be positively correlated with infection risk, as increasing species diversity will result in increased abundance of host species, and therefore of inoculum sources for a focal host. Conversely, the "Dilution Effect" hypothesis posits that reduced species diversity increases the abundance of the focal host species, facilitating pathogen transmission and increasing infection risk. Thus, higher species diversity decreases the abundance of the focal host, resulting in a reduction in the number of contacts between susceptible and infected individuals and in lower infection risk (Keesing et al., 2006; Ostfeld and Keesing, 2012).

Most experimental analyses on the effects of plant diversity and relative abundance on infection risk have focused on the effects of plant species richness on the prevalence of foliar fungal diseases (e.g. Mitchell et al., 2002; 2003; Roscher et al., 2007). The majority of these studies indicated that higher species richness reduced infection risk due to a lower host density or to the differential ability of host species to transmit the pathogen ("Dilution Effect"). Investigations about the relationship between host species diversity and infection risk in plant-virus systems are also scarce and restricted to a handful of interactions (Malmstrom et al., 2005a, 2005b; Pagán et al., 2012; RodeloUrrego et al., 2013). Interestingly, these works yielded contradictory results: data on infection of Cereal- and Barley yellow dwarf viruses (C/BYDV) in Poaceae species supported the "Amplification Effect" (Malmstrom et al., 2005a, 2005b). However, the effect of plant species diversity on begomovirus infection risk in the focal host Capsicum annuum var. glabriusculum (or wild pepper) 
accorded to the "Dilution Effect" (Pagán et al., 2012; Rodelo-Urrego et al., 2013).

\subsubsection{Community composition}

Further developments of the dilution and amplification theories proposed that which of these effects occurs in a given ecosystem would depend on factors other than to host species diversity or abundance (Randolph and Dobson, 2012; Johnson et al., 2015). For instance, the potential to increase infection risk and/or pathogen transmission might also depend on the identity of the species present in a given ecosystem (community composition) (Seabloom et al., 2009a, 2009b). If the most competent host species (i.e., those that better transmit the pathogen) are the most resilient to extinction, then infection risk will increase as biodiversity declines. On the other hand, if competent host species tend to disappear as diversity declines, then the loss of species diversity will tend to reduce infection risk. Also, if increasing species diversity leads to the introduction of new host species, the resulting scenario would be compatible with the "Amplification Effect", whereas if new species were mostly non-hosts a "Dilution Effect" would be more likely (Randolph and Dobson, 2012; Johnson et al., 2015). On this regard, the life cycle of the species present in an ecosystem could be a relevant trait to analyze (Johnson et al., 2012). Theoretical work has proposed that short-lived (annuals) hosts are better pathogen reservoirs than long-lived ones (perennials). Long-lived species are more likely to be challenged against pathogen infections during their life span, and therefore invest in developing defense mechanisms. Conversely, short-lived hosts will invest less in costly defenses against pathogens, so that they will be more susceptible to infection and/or will sustain larger vector populations, thus being effective reservoirs for infectious diseases (Boots and Bowers, 2004; Miller et al., 2007; Hily et al., 2014; Bruns et al., 2015). The local abundance of longlived hosts has been also associated with increased infection risk: long-lived hosts can increase pathogen spread rates and prevalence by serving as longterm sources of infection for susceptible individuals (Herms and Mattson, 1992; Borer et al., 2010). This effect of the plant lifespan on infection risk may differ 
between wild and agroecosystems as, at odds with most agroecosystems, wild ecosystems are dominated by perennials (Malmstrom et al., 2011).

Few studies have directly or indirectly investigated the importance of host plant life cycle on infection risk, and the majority was done under laboratory conditions. Salvaudon et al., 2013 showed that long-lived genotypes of Arabidopsis thaliana were more tolerant to Hyaloperonospora arabidopsidis, indirectly showing that hosts with longer life spans were more likely to be better sources of pathogen inoculum. More directly, Hily et al., 2014 demonstrated that long-lived $A$. thaliana genotypes were better reservoirs of Cucumber mosaic virus (CMV) than short-lived ones. There is also evidence that, in wild ecosystems, short-lived (annual) species may have a high reservoir potential of a generalist plant virus than long-lived (perennial) hosts (Malmstrom et al., 2005b; Alexander et al., 2017). Therefore, the relationship between lifespan of plant species and pathogen infection risk remain only partially understood.

\subsubsection{Host density and biomass}

Because host density affects directly host-pathogen encounter rates, the density of the host plant populations can have a marked effect on the size and rate of increase of those of their pathogens. Indeed, an accepted axiom in plant pathology is that a greater host density would reduce the distance between susceptible hosts, facilitating the dispersion of pathogens and increasing infection/disease risk (Burdon and Chilvers, 1982). Agroecosystems are homogenous environments with higher host density that facilitates pathogen transmission between plants, driving the emergence of highly virulent, hostspecialized plant pathogens compared to pathogens in wild ecosystems. Therefore, in agroecosystems host density is expected to have a central role in infection risk, and most evidence supporting this positive association between host density and infection risk comes from studies in agroecosystems (Thrall and Burdon, 1999; Burdon and Thrall, 2008; Stukenbrock and McDonald, 2008). However, it should be noted that evidences for a negative correlation between host density and disease risk also exist (reviewed by Burdon and Chilvers, 1982). 
Few studies have addressed the association between plant density and infection risk in wild ecosystems. Many of these have recorded a positive association between the two traits (Alexander, 1984; Burdon et al., 1992; Knops et al., 1999; Mitchell et al., 2002; Cobb et al., 2010; Pagán et al., 2012). However, the influence of many interacting factors in the ecosystem makes it difficult to interpret the effects of host plant density on disease risk (Power, 1987). For example, the effects of species biodiversity and host density on disease risk are often difficult to differentiate (Keesing et al., 2010). On this regard, it has been shown that infection risk increased with biodiversity loss associated with increased host plant density (Knops et al., 1999; Cobb et al., 2010). The interpretation of most works in wild ecosystems is limited by the difficulty of disentangling these interactions, and only a few utilized methodologies to separate such effects (Pagán et al., 2012).

As for plant biomass, some authors have proposed that higher biomass may increase the prevalence of fungal pathogens because it favors conditions for pathogen development (Harvell et al., 2002). For viruses, larger plant biomass may favor contact between plants and promote the growth of vector populations increasing virus prevalence (Sacristán et al., 2004). However, very few works have empirically tested these ideas, and with contradictory results. While plant biomass was positively associated with fungal growth (McCartney, 1997) and with virus prevalence (Sacristán et al., 2004), other works failed in finding any effect of biomass on infection risk (Seabloom et al., 2010).

\subsubsection{Pathogen host range}

Emergence is not only influenced by host ecology, but also by pathogen ecology. One the major pathogen traits that could influence emergence is its host range, i.e., the number of species that a pathogen can infect. Pathogens can be divided into two groups according to their host range: generalists and specialists. Generalist pathogens infect many host species, often from taxonomically distant plant families (Whitlock, 1996), an ability that provides such pathogens with more chances of survival and transmission. On the other hand, specialists are able to infect one or few closely related host species, where these pathogens are highly transmissible and efficiently exploit host 
resources (Futuyama and Moreno, 1988). Because biological fitness varies across environments, virus fitness shall vary across host species (Elena et al., 2014). As a consequence, it is often assumed that generalism comes at the cost of suboptimal virus transmission and host resource exploitation, as generalist viruses cannot maximize their fitness in all hosts. On the other hand, specialist pathogens will overcompete generalists in the host that the former can infect, but at the cost of a high dependency on the availability of such host (Pagán et al., 2016). Which of this two strategies is "selected" by a pathogen may deeply impact how host ecology affects infection risk. It has been proposed that the "Amplification Effect" would require a generalist pathogen that infects a wide host range. Otherwise, increasing of the number of species in an ecosystem would rarely increase the number of host species. Following this rationale, the more restricted the host range of the pathogen, the higher the "Dilution Effect".

Remarkably, few studies have compared how host ecology affects the infection risk of generalist and specialist pathogens. For plant viruses, the relationship between biodiversity and disease risk has been analysed using the generalist C/BYDV, with results that are compatible with the "Amplification Effect" (Malmstrom et al., 2005a, 2005b; Borer et al., 2010). On the other hand, a decrease in biodiversity was associated with an increased risk of infection in wild pepper populations by two specialist viruses, the begomoviruses Pepper golden mosaic virus (PepGMV), and Pepper huasteco yellowvein virus (PHYVV) supporting "Dilution Effect" hypothesis (Pagán et al., 2012). However, in these wild pepper populations the relationship between the risk of infection by CMV, a generalist virus, and plant species diversity also accorded to the "Dilution Effect". It should be noted that these works defined host range according to literature, and did not attempted to characterize the virus host range in the analysed ecosystems. It has been recently shown that virus host range may differ according to habitat, such that theoretical generalists may act as specialists in certain conditions (McLeish et al., 2017). Thus, in order to understand how pathogen host range affects infection risk, the host range of the pathogen under study in the analysed ecosystems should be known. 


\subsubsection{Abiotic factors}

The process of pathogen emergence does not depend only on biotic factors. In an ecosystem, plant-virus-vector interactions occur also within an abiotic environmental context. Thus, it is expected that climatic conditions and other abiotic factors that define an ecosystem also have a fundamental role in infection dynamics (Patz et al., 2000). Moreover, the current situation of climate change likely alters the interaction between hosts, viruses and their vectors. Therefore, it is of particular importance to investigate how changes in climatic conditions affect pathogen infection risk (Anderson et al., 2004). However, environmental mediation of infection/disease risk is only partially understood. Temperature, relative humidity, rainfalls and edaphic factors have been proposed as relevant factors affecting pathogen emergence. Variation in the abiotic environment can mediate infection risk through: (i) their effect on host physiology, altering host defense responses and predisposing plants to infectious diseases, and (ii) their effect on population dynamics of vectors, promoting pathogen transmission (Garret et al., 2006; Kennelly et al., 2012).

\subsubsection{Effect of abiotic factors on host physiology}

Host plants are susceptible to changes in abiotic environmental conditions and, if these changes result in weakened individuals, plants may be less able to fence against pathogen infections or vector attacks (Kennelly et al., 2012). It has long been recognized that temperature is one such abiotic factor (Kassanis, 1957). Research that focused on the molecular mechanisms underlying the effect of temperature on disease dynamics found that temperature determines the efficacy of plant defense mechanisms such as RNA silencing and genetic resistance. For example, antiviral resistance mechanisms based in proteinprotein recognition of the pathogen by the plant are also temperaturedependent (Canto and Palukaitis, 2002; Pfitzner, 2006). It is well known that temperature above $28^{\circ} \mathrm{C}$ results in the inactivation of the genetic resistance conferred by the $\mathrm{N}$ gene against Tobacco mosaic virus (TMV) (Canto and Palukaitis, 2002). Also, Zhang et al., 2012 showed that higher temperatures resulted in increased replication of Turnip crinkle virus (TCV) in Arabidopsis plants due to the accumulation of loss-of-function mutations in proteins involved 
in the RNA silencing-based defence. Other studies have shown that plants are more susceptible to viruses at low temperature (more severe symptoms) due to a less efficient activity of the RNA silencing-mediated plant defense (Tuttle et al., 2008). Other climatic conditions have been also shown to affect plant infection/disease dynamics. An example of rainfalls mediation of pathogen prevalence has been reported using C/BYDV-infected natural grasslands. This study showed an indirect effect of the rainfalls regime on both host fecundity and virus prevalence: in sites with lower precipitations, virus prevalence increased, and authors speculate that precipitations may have indirectly increased virus prevalence by increasing host stress (i.e., drought stress) beyond the stress level caused only by virus infection (Seabloom et al., 2009).

The impact of soil edaphic factors can also influence infection/disease risk by modulating traits of hosts and pathogens involved in pathogen spread. It has been shown that, after seeding, soil moist conditions and possibly soil type favor survival of CMV seed-infected plants. Higher seed survival would result in a greater source of primary virus inoculum available for acquisition and spread to susceptible plants by aphids (Bwye et al., 1994; Bueso et al., 2017). Also, variability in soil moisture and/or soil type can limit nutrient availability and determine the diversity and composition of communities of plants in the ecosystems (Borer et al., 2014a, 2014b), therefore defining the number and identity of host species present in a given ecosystem.

\subsubsection{Effect of abiotic factors on the vector population}

Seasonal variation of temperature, relative humidity and rainfalls have been repeatedly reported to have a great effect in the abundance of aphid population and other virus vectors because of their impact on vector reproduction and ability to transmit viruses (Morales and Jones, 2004; Fajinmi et al., 2011). For example, it has been observed that seasonal changes in temperature and humidity affected the population size of thrips (Frankliniella fusca and Frankliniella occidentalis), vectors transmitting Tomato spotted wilt virus (TSWV): high humidity and low temperatures negatively affected thrips populations by killing larvae. This study also reported an increased flight activity of adult vectors at higher temperatures and lower relative humidity, which was 
associated with higher TWSV prevalence (Culbreath and Srinivasan, 2011). Similar effects of temperature and relative humidity on the reproductive ability, mortality and flight activity of aphid populations have been reported (e.g. Díaz and Fereres, 2005).

Several works aimed at developing mathematical predictive models of pathogen emergence have recognized the importance of climatic factors in determining pathogen infection/disease risk. Many of these works showed that simulating vector population dynamics as a function of seasonal changes in temperature, humidity and rainfalls significantly improves prediction of disease emergence (Mohasin and Chona, 2002; Thackray et al., 2004; Reynaud et al., 2009; Chappell et al., 2013). These models have been mostly developed for crop pathogens and very few works have analysed how co-occurring stress factors, (i.e., plant pathogen infection together with adverse climatic conditions), influence infection risk in wild ecosystems (Sacristán et al., 2004; Cooper and Jones, 2006).

\subsection{Genetic diversity of plant virus populations}

The process of pathogen emergence, including viruses, does not only involves changes in pathogen epidemiology, but also in the genetic composition of the pathogen population. These changes are thought to be dependent of the ability of the virus to generate genetic diversity, as well as on ecological factors that may shape the genetic diversity of the virus population (Elena et al., 2014).

\subsubsection{Generation of genetic diversity in plant virus populations}

One of the defining characteristics of viruses is their high capacity to generate genetic diversity (Holmes, 2009). Genetic diversity is the essential component in a population that allows a species to evolve in an everchanging environment with shifting selection pressures. A fundamental step to reduce the number of emergent virus diseases, and a long-standing goal for evolutionary biologists, is to understand which factors modulate the genetic diversity of virus populations. As for most organisms, the two main mechanisms for generating genetic diversity in plant virus populations are mutation and recombination. 


\subsubsection{Mutation}

The high capacity to generate genetic diversity derives from a combination of factors. The first major factor is the high mutation rates of plant viruses. Most plant viruses have RNA genomes that encode RNA-dependent RNA polymerases, which lack proofreading activity resulting in high rates of nucleotide misincorporation (Drake and Holland, 1999). Mutation rates in RNA plant viruses, first estimated for Tobacco mosaic virus (TMV) (Malpica et al., 2002), are in the range of $10^{-3}$ to $10^{-6}$ nucleotide substitutions per site per round of replication, similar to those reported for RNA viruses infecting bacteria or animals (Malpica et al., 2002; Sanjuán et al., 2009, 2010; Tromas and Elena, 2010). Thus, viral mutation rates are several orders of magnitude higher than those of their host plants, estimated to be around $10^{-9}$ (Kay et al., 2006). High mutation rates allow viruses to explore large portions of the mutational space. The higher the mutation rate the larger the probability to generate virus genotypes fitter in new environments. However, it has been well established that most mutations in RNA plant viruses are highly deleterious, with an important fraction being lethal, whereas neutral mutations are considerably less frequent (Carrasco et al., 2007; Hillung et al., 2015). In this scenario, high mutation rates may lead to a high burden of deleterious mutations (i.e., mutational load) in the virus populations and ultimately to extinction (Chao, 1990). Here, the second major factor associated with the high genetic diversity of virus populations becomes important: plant viruses generally have large population sizes (García-Arenal et al., 2001). For instance, the number of infectious units of TMV has been estimated as about $10^{7}$ per infected mesophyll cell of experimentally infected tobacco plants (Harrison, 1956; Malpica et al., 2002), and the number of Tobacco mild green mosaic virus (TMGMV) particles in field-infected Nicotiana glauca leaves has been estimated as $10^{11}$ (Moya et al., 1993). Also, the effective population size of TMGMV in $N$. glauca was estimated to be of about $10^{5}$ (Moya et al., 1993), and that of 12 potyviruses have been estimated to be in the order of $10^{4}$ (Hughes, 2009). As a consequence, deleterious mutations have small chances to persist in the virus population, as these will be quickly purged by negative selection (Elena and Sanjuán, 2005). However, it should be noted that factors, such as variation in 
replication potential among genotypes, differences in generation time among infected cells, and severe reductions in population size at various steps during the virus life cycle, might lead to effective population sizes (roughly, the number of individuals in the population that pass their genes to the next generation) much smaller than the census size of the population. Finally, the third major factor is the much shorter generation time of RNA viruses (minutes to hours) (Wu et al., 1994) than that of plants. Thus, virus evolution occurs in different time scales from that of their host plants.

\subsubsection{Recombination}

Besides mutation, genetic diversity in virus populations can also be generated by recombination, that is, the exchange of genomic fragments between genotypes (Simon-Loriere and Holmes, 2011). Recombination can occur between genotypes of the same or of different virus species. Recombination rates have been estimated for plant viruses to be between $10^{-5}$ and $10^{-8}$ (Froissart et al., 2005; Simon-Loriere and Holmes, 2011; Tromas et al., 2014), so that the contribution of recombination to the generation of genetic diversity would be in the same order as that of mutation. Recombination may represent an evolutionary advantage for viruses because (1) it can create fitter genotypes more rapidly than mutation and (2) it might purge deleterious mutations from virus populations, thereby preventing the decrease in overall fitness in clonal populations due to the accumulation of deleterious mutations (Muller's ratchet) (Pressing and Reanney, 1984; Chao, 1990; García-Arenal et al., 2001; Hull, 2014; Moya et al., 2004). Thus, genetic exchange by recombination may have important epidemiological implications of practical relevance, and it has been associated with host jumps, host range expansion, changes in virulence, breaking of host resistance and finally, the emergence of new viral plant diseases (Pita et al., 2001; Díaz-Pendon et al., 2010).

\subsubsection{Processes that shape the genetic diversity of plant virus populations}

Mutation and recombination are a consequence of the mechanisms of virus replication, and the resulting new genotypes are therefore in principle randomly generated (but see Bujarski, 2013 for exceptions). Central to understanding the 
population genetic diversity of plant viruses is which mutations become fixed in the population and how fast they do so (Duffy et al., 2008) or, more generally, what determines the frequency in the populations of the genotypes generated through mutation and recombination. In the absence of migration, the number and frequency of these genotypes in the population (i.e., the genetic structure of the population) is the result of two different evolutionary processes: genetic drift and selection (García-Arenal et al., 2001; Hartl and Clark, 2007; Acosta-Leal et al., 2011).

Genetic drift occurs when populations of organisms are not large enough to ensure that each genotype will have progeny in the next generation. As a consequence, the genotypes passed into the next generation are randomly sampled from the mother population, regardless of their relative fitness. Genetic drift may be particularly relevant in plant virus populations during the severe reductions in their population size (population bottlenecks) that may occur along the virus life cycle, for instance, at the infection of a new host population, a new host plant, or new organs within a host plant (Sacristán et al., 2003; Gutiérrez et al., 2012; Fabre et al., 2014). Genetic drift reduces the genetic diversity of populations and increases the diversity among populations. Also, because the genotypes that start a new population are not selected according to their fitness, genetic drift counters the effects of selection (García-Arenal et al., 2001; Acosta-Leal et al., 2011).

On the other hand, selection is a directional process by which genotypes that are fittest in a given environment will increase in frequency in the population (positive or diversifying selection), whereas less fit genotypes will decrease in frequency (negative or purifying selection). As is the case for genetic drift, selection results in a decrease of the population diversity and may also cause an increased diversity between populations, if under different selection pressures, so that the effects of selection and genetic drift are often difficult to distinguish. When selection has been differentiated from genetic drift, selection has been associated with every life history trait of plant viruses, such as survival in the environment due to higher structural stability of the virus particles (Fraile et al., 2014), adaptation to the host plant resulting in more effective within-host multiplication (Hillung et al., 2015), and adaptation to the transmission mode resulting in more efficient between-host transmission 
(Hajimorad et al., 2011; Pagán et al., 2014). These processes are associated with the colonization of new geographical areas and have been proposed as a possible explanation to the emergence of viral diseases in crops (Marco and Aranda, 2005; Moriones and Navas-Castillo, 2008). Sequence analyses of plant virus genomes indicated that selection on the encoded proteins is mostly negative (García-Arenal et al., 2001). This implies that selection plays an important role to restrict diversity (Schneider and Roossinck, 2001).

Although there is a large number of studies that analyze the genetic diversity and structure of plant virus populations, very little is known about what environmental factors shape such genetic diversity and how they affect the process of emergence (Fraile and García-Arenal, 2016).

\subsubsection{Ecological factors affecting virus population genetic diversity}

Ecological changes that affect infection risk may also result in genetic changes in the virus population (Grenfell et al., 2004; Archie et al., 2009; Pybus and Rambaut, 2009). Indeed, theoretical elaborations hypothesize that biodiversity may determine the genetic diversity of pathogen populations, including viruses, through changes in the pathogen's epidemiology. According to this hypothesis, the loss of biodiversity would increase host abundance and density, which in turn would lead to higher transmission rates and a higher prevalence of pathogens (Keesing et al., 2006; Burdon and Thrall, 2008; Keesing et al., 2010; Ostfeld and Keesing, 2012). As a consequence of these epidemiological changes, pathogen populations would increase in size, accelerating their evolutionary rates (Scholle et al., 2013; Lanfear et al., 2014), which could ultimately lead to higher genetic diversity.

As shown in the previous sections, there is increasing evidence linking the loss of ecosystem biodiversity to higher transmission rates and prevalence of viruses and other pathogens (reviewed in Keesing et al., 2010; Ostfeld and Keesing, 2012; Alexander et al., 2014; Pagán et al., 2016). However, the understanding on how the loss of biodiversity modulates the genetic diversity of virus populations is scarce and relies mostly on indirect evidence (Holmes, 2009; Scholle et al., 2013; Lanfear et al., 2014). This is especially so in the case of plant viruses (Alexander et al., 2014), for which most of the existing evidence 
comes from analyses of plant-virus interactions under different levels of ecosystem anthropization, which is also the major cause of biodiversity loss (Jones, 2009; Keesing et al., 2010). At odds with the hypothesis above, comparative genomics analyses of geminivirus species showed a reduction of virus genetic diversity and of recombination rates with increased ecosystem anthropization (Silva et al., 2011, 2012; Lima et al., 2013). On the other hand, inferences of divergence times for several groups of plant viruses have traced their radiation to the origin or the intensification of agriculture (Fargette et al., 2008; Duffy and Holmes, 2008; Gibbs et al., 2008; Pagán and Holmes, 2010, but see Lefeuvre et al., 2010; Yasaka et al., 2014). These works proposed that such bursts of genetic diversity could have co-occurred with the intrinsic loss of biodiversity characteristic of agroecosystems. Altogether, these results suggest that the effect of ecosystem anthropization, and the concurrent biodiversity loss, on the genetic diversity of plant viruses may depend on the relative importance of mutation and recombination. In agreement with this idea, Rodelo-Urrego et al., 2015 showed that, in wild pepper-infecting begomoviruses, recombination was more important as a mechanism of generating genetic diversity in habitats with higher biodiversity than in those with lower biodiversity. These apparently contradictory results mostly derive from analyses that combine viral sequences obtained from different hosts, and from different geographic locations. Hence, it is difficult to disentangle whether the reported changes in plant virus genetic diversity are indeed due to ecosystem biodiversity or the result of host and/or local adaptation processes. As a consequence, it is still unclear how biodiversity loss affects the genetic diversity of plant virus populations.

\subsubsection{Virus emergence and speciation}

The process of genetic diversification of the virus population may result in the emergence of new diseases caused by the appearance of new virus species (i.e. "speciation" events) (Huyse et al., 2005; Elena et al., 2011). Viral speciation can occur either sympatrically or allopatrically. Sympatric speciation would occur when viruses diversify and adapt to different niches within the same host species (maybe using different cell types, establishing different seasonalities, etc). In contrast, allopatric speciation represents the genetic diversification that 
occurs in different host species, either by cross-species transmission (i.e., host jumping) or codivergence with hosts over extended time periods (Kitchen et al., 2011).

Evolutionary processes at two levels have been proposed to equally determine the mode of virus speciation: "microevolutionary" processes, that is, those that shape intra-species and intra-host genetic diversity, and "macroevolutionary" processes, i.e. those affecting inter-host or inter-species genetic diversification (Huyse et al., 2005). Both genetic and ecological factors are thought to shape these processes (Huyse et al., 2005; Pagán et al., 2016). However, to date only a reduced number of studies have analysed speciation at both scales. Most of these works have focused in fungi and bacteria (Buckling and Rainey, 2002; Giraud et al., 2010), and few in host-virus systems (Pagán and Holmes, 2010; Kitchen et al., 2011). Thus, analysis of plant virus speciation and the role of ecological factors involved at both scales remains under investigated, particularly in wild ecosystems.

\subsection{Wild ecosystems in the Iberian Peninsula}

The Iberian Peninsula $\left(582,000 \mathrm{~km}^{2}\right)$ is located in the southwest corner of Europe. From a biogeographical perspective, the Iberian Peninsula can be divided into two regions with differentiated ecological features, Eurosiberian and Mediterranean (Rivas-Martínez, 2004) (Figure 1.2).

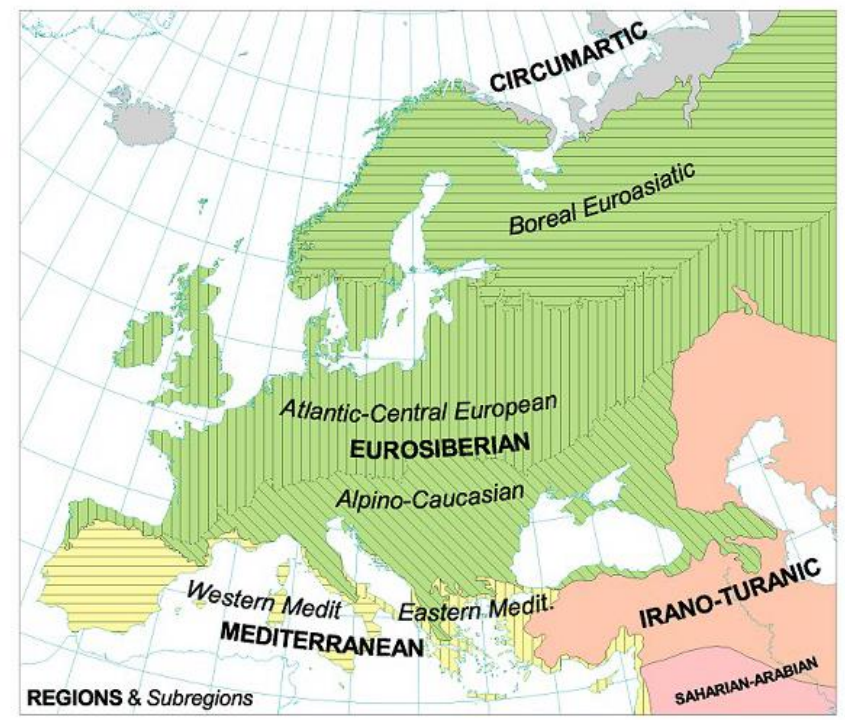

Figure 1.2. Biogeographic map of Europe (Rivas-Martínez et al., 2004). 
The Eurosiberian region covers the northern third of the peninsula, which is under the influence of a mild and humid Atlantic climate. The Mediterranean region spans the remaining territory and is characterized by a Mediterranean climate with significantly drier and warmer conditions than the Eurosiberian region (Rivas-Martínez, 2004).

As a consequence of its biogeographical location, in the transition between the Eurosiberian and Mediterranean regions, the Iberian Peninsula has remarkable forest diversity. There is a wide knowledge of the Iberian ecosystems in terms of its vegetation structure and climate-based floristic composition (e.g. Gavilán and Fernández-González, 1997; Gavilán et al., 1998; Gavilán, 2005; Rivas-Martínez, 2007). Particular biotic strategies predominate in the forests of each of these two biogeographical regions (Figure 1.3).

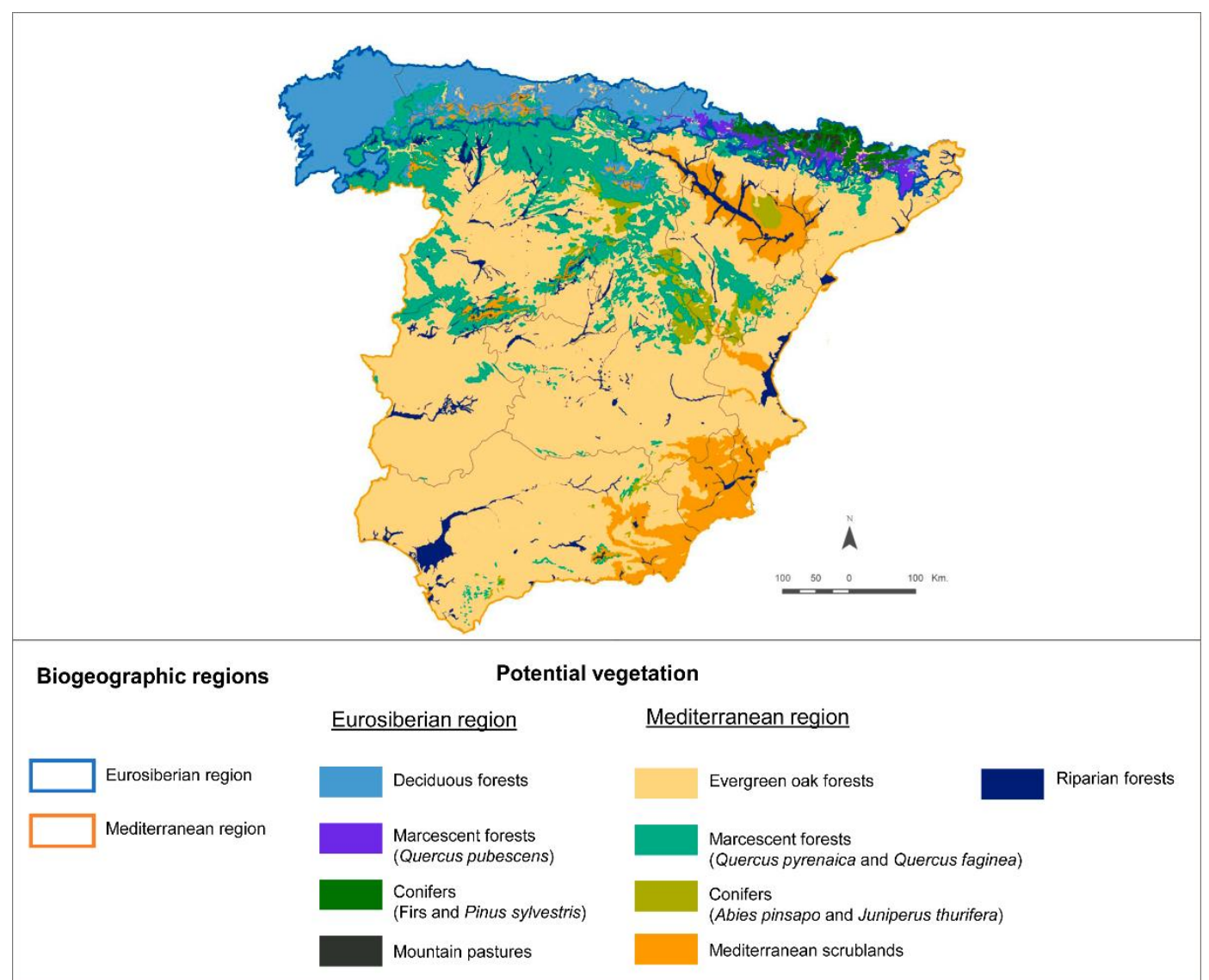

Figure 1.3. Map of potential vegetation in Spain. Modified from "Mapa de Series de Vegetación de España” (Rivas-Martínez, 1987). Departamento de Geografía y Ordenación del Territorio. Universidad de Zaragoza.

The forests of the Eurosiberian region are determined by the need of overcoming the winter cold that constitutes the most unfavorable factor. Thus, 
Eurosiberian forests are dominated by deciduous species. On the other hand, the conditions of the Mediterranean region favor complex mosaics of plant communities of evergreen, deciduous and/or mixed forests, scrublands or natural grasslands. In this region, rainfalls are irregularly distributed over time. Thus, xerophytic perennial plant species adapted to survive long periods of drought have achieved greater success (Blanco et al., 2005). Within the Mediterranean region, there are patches of Atlantic climate occupying particularly humid areas for microclimatic or edaphic reasons, specifically in mountain areas or river basins. These Atlantic patches have the capacity to allow the introgression of plant species typical of humid climatic regions into more arid ones (Stella et al., 2013) (Figure 1.3).

According to the Coordination of Information on the Environment (CORINE) Agency Land Cover Map of 2012 (Figure 1.4), more than a third $(34 \%)$ of Spain territory is covered by croplands, while almost another third $(26 \%)$ is covered by forested lands. Semi-natural vegetation, that includes shrub and/or herbaceous vegetation associations, represents $21 \%$ of the total, followed by pastures (13\%), while the least common forms of cover are water areas $(1 \%)$ and wetlands $(0.2 \%)$.

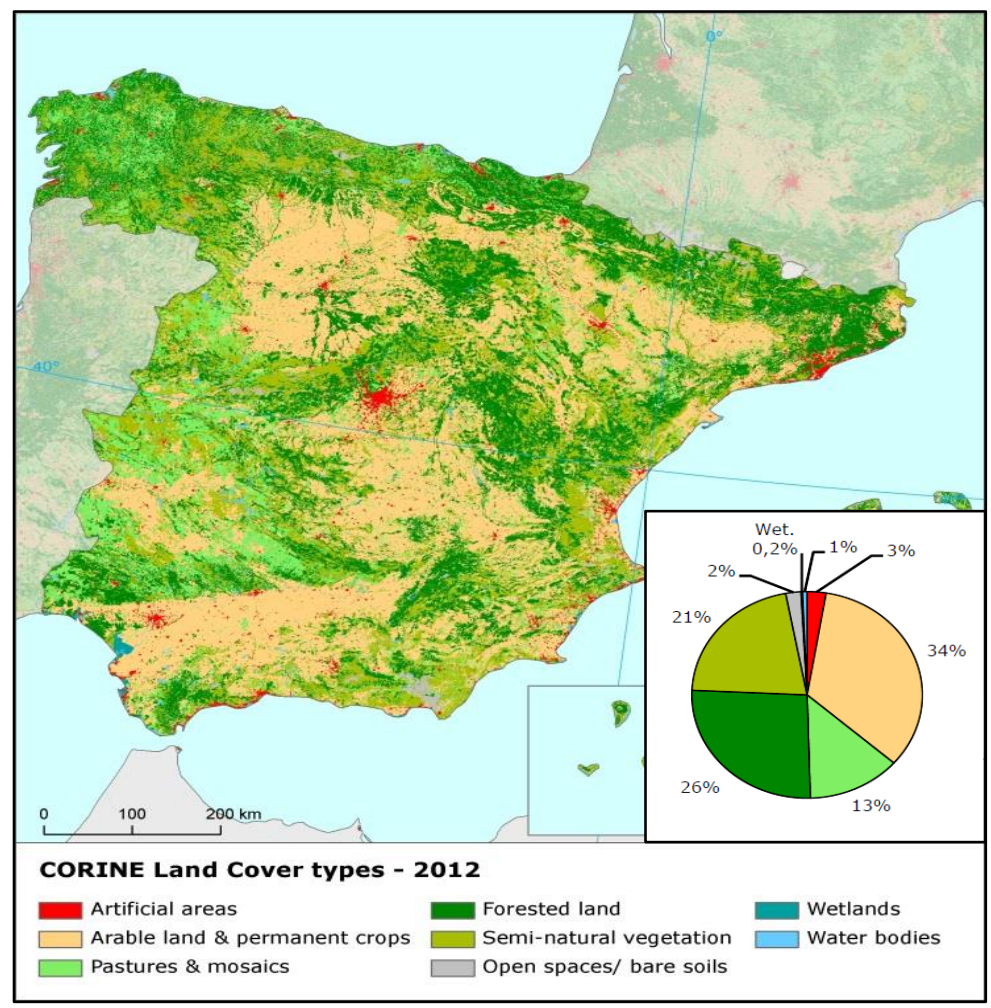

Figure 1.4. Map and percentage of land cover types in Spain. According to CORINE (Coordination of Information on the Environment), 2012 (https://www.eea.europa.eu). 
This thesis focused on the Mediterranean biogegraphical region, which is the larger in the Iberian Peninsula (Figure 1.3). Specifically, the area studied is located in central Spain (Meseta). The Meseta is a large plateau situated at 600 $\mathrm{m}$ of elevation, and is traversed by the Spanish Sistema Central, a mountain range that divides the Duero and Tajo watersheds. As mentioned above, this region is under a Mediterranean-Continental climate, which is characterized by extreme temperatures and strong diurnal and seasonal thermal oscillations. Precipitations in this climate are irregular, these being more frequent in spring and autumn, and very scarce in summer with high rates of evaporation that leave the land arid (Loidi, 2017). The thesis focuses on two forest types in this region: evergreen oak forests and riparian forests. The former is the most representative and abundant forest in the Mediterranean region of the Iberian Peninsula; the later forest is representative of the Atlantic climate patches embedded in the Mediterranean region. Their distribution and their different environmental conditions influence the adaptive responses, structure and floristic composition of plant communities in both types of forests. Hence, they are characterized by diverse ecological conditions in terms of biodiversity, floristic composition and climatic conditions (Blanco et al., 2005) that, as mentioned previously, can potentially affect plant virus infection risk and virus population genetic diversity. Thus, these forests represent a suitable system to analyse how ecological conditions affect pathogen emergence. Moreover, both types of forests are frequently bordering crop fields (Figure 1.4). Thus, these two ecosystems are potential sources of inoculum for the emergence of diseases in the crops. Therefore, understanding the ecological factors that favor the emergence of viral diseases in evergreen oak and riparian forests may provide relevant information for the development of conservation policies, and also for the prevention of emerging diseases in crops.

\subsubsection{Riparian forest}

Riparian forests typically appear adjacent to riverbeds (Figure 1.3). The presence of the river causes important changes in the ecological conditions of the adjacent lands. The most remarkable one consists of a greater water availability, which leads to an increased relative humidity as a consequence of 
higher evapotranspiration, higher soil moisture, and particular soil textures $/ \mathrm{pH}$. There are also changes in the thermal regime: the maximum temperatures are attenuated due to the higher energy consumption in the evapotranspiration process. These conditions allow riparian forests to avoid the consequences of the summer drought characteristic of the Mediterranean climate. As a consequence, in this forest lives vegetation typical of the Atlantic environments, along with the riparian vegetation of Mediterranean distribution (Blanco et al., 2005). This partly explains the high floristic diversity and the structural complexity of Mediterranean riparian forests (Stella et al., 2013).

The diversity of plant species found in riparian forests is generally organized according to the distance to the riverbed and therefore to the amount of water available. So that, the plant communities most specialized will happen on the water and near the riverside; families such as the Ranunculaceae, Juncaceae, Cyperaceae or grasses such as the reed (Phragmites australis) among others, constitute the dominant herbaceous stratum in this sector. Also, communities of clovers (e.g. Trifolium pratense, Trifolium campestre) develop together with other species such as Rumex crispus, Rumex conglomeratus and mints (e.g. Mentha longifolia). Among the vegetation of the shrub layer, members of the family Rosaceae such as Crataegus, Rosa or Rubus species are also important. Also near to the river course, arboreal and/or shrub vegetation (e.g., Salix eleagnos, Salix cinerea) resistant to flooding can be found contributing to the fixation of fluvial deposits. Finally, forests linked to conditions of greater climate stability on the riverbed, but less dependent on water, are composed mainly of deciduous trees as poplars (Populus nigra), willows (Salix atrocinerea), alders (Alnus glutinosa), ash (Fraxinus angustifolia), elm (Ulmus minor) and sometimes by Pyrenean oak (Quercus pyrenaica). Climbing plants play also an important role in the structure of riparian vegetation and reach great diversity and development due to the more humid environmental conditions. Some examples among this diversity are Hedera helix, Humulus lupulus, Lonicera periclymenum subsp. hispanica, Rosa sempervirens, Rubus spp., Solanum dulcamara, Tamus communis or Vitis vinifera subsp. Sylvestris (Blanco et al., 2005).

The riparian vegetation communities delay the speed of water in floods, protect the stability of adjacent lands, and enable the deposit of nutrients 
transported by water, thus contributing to increase the soil fertility and the development of higher plant density and biomass. As a consequence, large parts of the remaining riparian forests of the Tajo and Duero rivers in central Spain are protected. Still, the lands of riparian influence are often used for the installation of orchards, pastures and arboreal/herbaceous crops of high yield, such that cultivated lands can be found near protected areas (Virgós, 2001).

\subsubsection{Evergreen oak forest}

Holm oak or evergreen oak (Quercus ilex L.) forests are widely distributed in the Mediterranean basin (Figure 1.5). Approximately $60 \%$ of this type of forest is located in the Iberian Peninsula where they represent the most characteristic forests (Ramírez and Díaz, 2008; Patón et al., 2010). In Spain, evergreen oaks dominate in about 3 million ha, i.e. $25 \%$ of the area where trees cover at least $10 \%$ of the ground (Rodà, 1999) (Figure 1.5). In the geographical areas dominated by evergreen oaks, plants have to cope with a selective pressure resulting from multiple climatic stresses: winter cold, irregularity in the rainfall distribution, and specially the coincidence of the period of maximum drought with the higher temperatures in summer. From the edaphic point of view, the lack of nutrients can also be a contributing stress factor. All these conditions have determined the morphological and physiological evolutive responses of the plant communities. These forests are formed mainly by broad-leaved sclerophyll vegetation, characterized by hard, evergreen foliage that is specially adapted to prevent moisture loss and to favor the storage of nutrients (Rodà, 1999).

The extreme conditions of the Mediterranean climate determine the structural and floristic composition of the evergreen oak forests in Central Spain. In this region, they are mainly monospecific forests with the dominance of holm oak trees in the arboreal stratum, which usually do not exceed $10 \mathrm{~m}$ in average height. Beneath the evergreen oak forest canopy, the influence of climate also causes a marked reduction of the species diversity, resulting in lower plant biomass than in riparian forests (Blanco et al., 2005; Stella et al., 2013). Among the most typical and abundant species under the canopy are: Daphne gnidium, the wild asparagus (Asparagus acutifolius), the gum rockrose 
(Cistus ladanifer), and Thapsia villosa (Blanco et al., 2005). The clearings of the evergreen oak forest have sandy soils and are very poor in organic matter. These clearings are occupied by different shrub formations adapted to sun exposure (heliophilous). Rockroses such as Cistus ladanifer in acid soils or Cistus laurifolius in mountain areas, and Retama sphaerocarpa in the most clayey soils becomes the predominant shrub element, being the most characteristic substitution stages of the evergreen oaks. When the soil degradation progresses, other shrubs such as the lavender (Lavandula stoechas subsp pedunculata) and thyme (Thymus mastichina) can be found along with the rockroses (Blanco et al., 2005). Also, clearings typically sustain Mediterranean annual plants. The most frequent species of these communities are Tuberaria gutatta and annual species of grasses, legumes and asteraceans that constitute the herbaceous stratum.

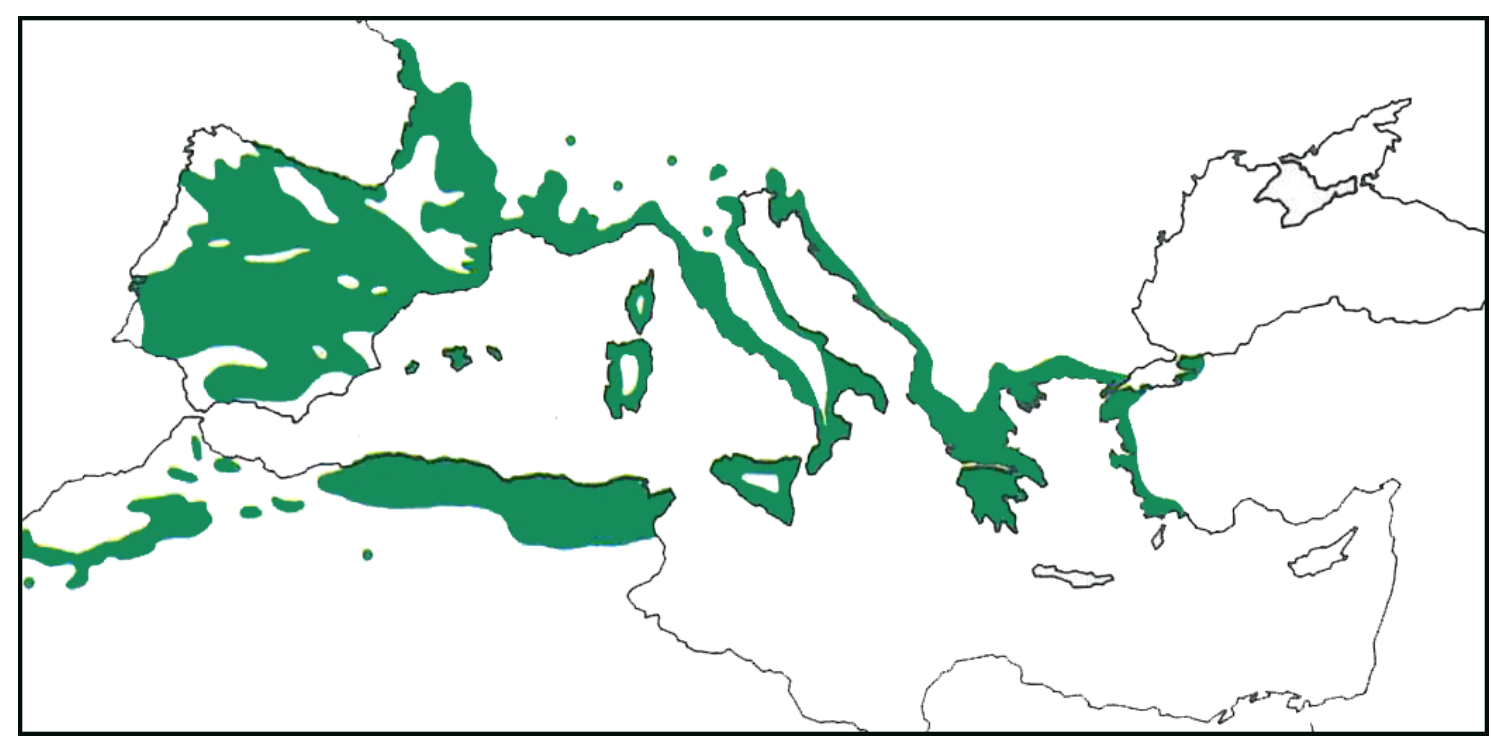

Figure 1.5. Distribution range of Quercus ilex in the Mediterranean basin (Blanco et al., 2005).

Evergreen oak forests have also great economic value, almost one million ha of the area occupied by these forests is used for firewood, and another 1.3 million ha are "dehesas", a savannah-type man-managed formation. In these formations, $Q$. ilex is considered as a fruit tree and has been selected for sweet acorn production, which is used to supplement feed for pigs (Pulido et al., 2001). In the remaining areas, evergreen oak forests can be regarded as woodlands that have undergone very low or no silvicultural management (Costa et al., 2011; Loidi, 2017), and that lay frequently adjacent 
to cultivated fields. This thesis focuses in this second type of evergreen oak forests as they can be considered as largely wild ecosystems.

\subsection{The genus Potyvirus}

The genus Potyvirus, named after its type member, Potato virus $Y$ (PVY), is the largest genus in the family Potyviridae, containing $90 \%$ of its species. Potyviruses are one of the two largest genera of plant viruses (represents $\sim 30 \%$ of all known plant viruses), the other being the begomoviruses (King et al., 2012). Viruses of the genus Potyvirus are characterized by common biological and genomic features: they form cytoplasmic inclusion $(\mathrm{Cl})$ bodies, are transmitted by vectors, share a genome structure and have similar nucleotide and amino acid sequence in the coat protein. Species and strains of this genus are differentiated on the basis of natural host range, pathogenicity and cytopathology, mode(s) of transmission, antigenic properties and genome sequence relatedness (Barnett, 1992). Replication of these viruses appears to be typical for (+) ssRNA viruses, where the plus strand is transcribed to produce a (-) RNA strand, which then serves as template for the production of progeny (+) strand RNA molecules. This process probably occurs in the cytoplasm (Mäkinen and Hafrén, 2014).

\subsubsection{Molecular biology}

Potyvirus virions are flexuous filaments, non-enveloped, rod-shaped particles of 680-900 nm long and 11-15 nm wide, with helical symmetry. Virions contain a single molecule of linear, positive sense single-stranded RNA (ssRNA), about 10,000 nucleotides, surrounded by about 2,000 units of coat protein (CP). The RNA genome carries a VPg (viral protein genome-linked) protein covalently bound to its 5'-end, which is encoded in the virus genome. The 3'-end of the genome is polyadenylated with a variable number of adenosines. The genome is characterized by a single major open reading frame (ORF) that encodes a polyprotein of about 340-370 kilodalton (KDa), which is subsequently cleaved by three virus-coded proteinases into ten functional proteins (Figure 1.6): the first protein (P1), the helper component protease (HC-Pro), the third protein 
(P3), the $6 \mathrm{~K} 1$ protein, the cylindrical inclusion protein $(\mathrm{Cl})$, the $6 \mathrm{~K} 2$ protein, the viral protein genome-linked ( $\mathrm{VPg}$ ), the small nuclear inclusion protein (Nla), the large nuclear inclusion protein ( $\mathrm{Nlb}$ ) and coat protein (CP). Gene order and protein sequences are conserved throughout the family Potyviridae (King et al., 2012) (Figure 1.6). Two additional proteins, P3N-PIPO and P3N-ALT, are originated through frameshifts in the P3 cistron (Chung et al., 2008; HagiwaraKomoda et al., 2016), although they are not present in all members of the genus.

A.

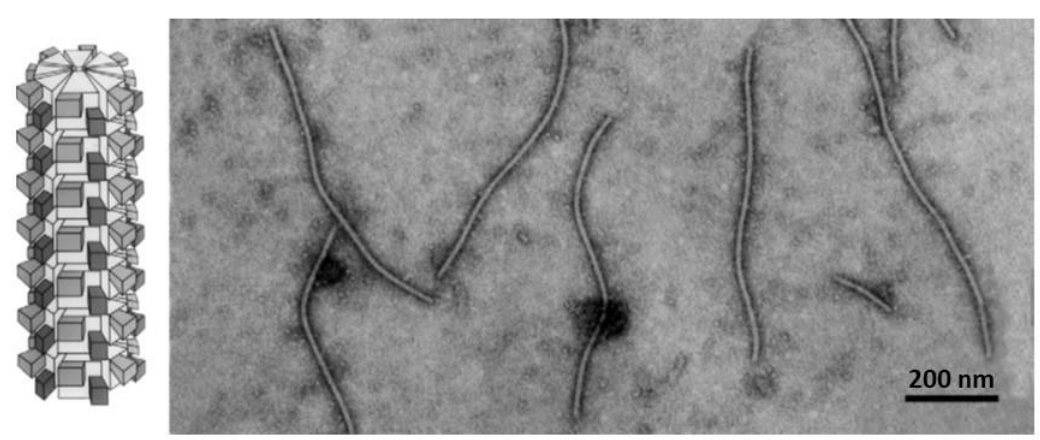

B.

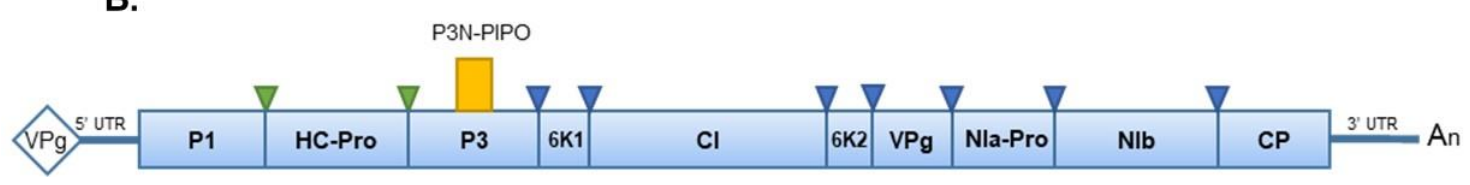

Figure 1.6. Potyvirus genome organization: A, Schematic diagram of a potyvirus particle (Left) and negative contrast electron micrograph of particles of an isolate of Plum pox virus (Right) (From King et al., 2012). B, genomic map of members of the genus Potyvirus (modified from Rodríguez-Nevado et al., 2017): The blue box represents the polyprotein ORF. The alternate P3N-PIPO ORF, depicted by the small yellow box above the main reading frame, is putatively translated by +2 frameshift of the polyprotein ORF, and its product is expressed as a fusion with the $\mathrm{N}$-terminal part of $\mathrm{P} 3$. Functions associated with the mature proteins proteolytically processed from the polyprotein are shown. Autocatalytic cleave sites of the HC-Pro and P1 gene products are indicated by green triangles, and Nla-Pro cleavage sites are marked by blue triangles. VPg, genome-linked viral protein covalently attached to the 5'-terminal nt.

Most of the mature virus proteins are multifunctional (Figure 1.6). The P1 corresponds to the $\mathrm{N}$-terminal gene of the potyvirus genome and encodes the first of three viral proteinases, cleaving the polyprotein at the P1/HC-Pro junction (Verchot et al., 1992). Together with P3, P1 is the least conserved 
protein among potyviruses, except for conserved amino acids found at the Cterminus of all potyvirus P1 proteins and which correspond to the proteinase catalytic domain. Recently, it has been demonstrated that the $\mathrm{N}$-terminal region of P1 controls early replication of potyviruses (Pasin et al., 2014). It has been also shown that the P1/HC-Pro fusion carries a potential pathogenicity enhancer, which is involved in suppression of host defense (Kasschau and Carrington, 1998; Rajamäki et al., 2005; Valli et al., 2007; Pasin et al., 2014). A new small ORF, called PISPO, has been identified inside the coding sequence of the P1-coding sequence of some potyviruses that infect sweet potato. This ORF could be translated by a frameshift similar to that involved in the expression of the PIPO ORF (Clark et al., 2012; Li et al., 2012). HC-Pro is the second viral proteinase, cleaving the polyprotein at the HC-Pro/P3 junction (Carrington et al., 1989). HC-Pro protein is known to suppress RNA silencing and stimulate viral genome amplification and long-distance movement (Kasschau et al., 2003; Soitamo et al., 2011). Recent results have revealed that HC-Pro is required to stabilize the CP for the proper yield and infectivity of potyviral progeny (Valli et al., 2014). Like P1, P3 has also been proposed to be involved in virus replication (Cui et al., 2010) and, together with 6K2, in virus movement and symptom development (Spetz and Valkonen, 2004). Based on the fact that the proteolytic splitting between P3 and $6 \mathrm{~K} 1$ is not essential for virus infectivity, it has been hypothesized that P3-6K1, rather than P3 and 6K1, might be the main functional product, and its proteolytic processing would have a regulatory role (Riechmann et al., 1995). However, processing at the P3-6K1 junction still affected symptom expression, suggesting that $6 \mathrm{~K} 1$ alone may has a relevant role in potyviral infection (Waltermann and Maiss, 2006). The $\mathrm{Cl}$ protein is a multifunctional protein (Sorel et al., 2014). This protein has ATPase and RNA helicase activities, which are required for virus RNA replication (Fernández et al., 1997). The Cl protein acts in collaboration with P3N-PIPO in aiding virus movement (Wei et al., 2010). Furthermore, the $\mathrm{Cl}$ protein acts as a virulence factor for different resistance genes (Sorel et al., 2014). The 6K2 protein is an integral membrane protein associated to $\mathrm{VPg}$ in endoplasmic reticulum-derived membranes (Schaad et al., 1997; Léonard et al., 2004) forming cytoplasmic vesicles which are viral replication sites (Cotton et al., 2009). Also, $6 \mathrm{~K} 2$ protein is found as a polyprotein with the VPg and the Nla 
protein (6K2-VPg-NlaPro product) in infected plants (Restrepo-Hartwig and Carrington, 1994). Nla is the largest protein and is partially processed to produce VPg and NlaPro (Dougherty and Parks, 1991). The free VPg is the major form of protein linked to the 5'-end of the genomic RNA although the complete $\mathrm{Nla}$ (VPg-NlaPro) has also been detected at the end of the RNA (Mathur and Savithri, 2012). When VPg is part of the Nla, it is localized both in the cytoplasm and in the nucleus of the infected cells (Beauchemin et al., 2007; Rajamäki and Valkonen, 2009) whereas when it is part of the 6K2-VPg-NlaPro product, VPg is targeted to membranous factories induced by the virus where it plays a key role in viral RNA replication (Beauchemin et al., 2007; Wei and Wang, 2008). VPg contains a nucleotide-binding motif and, when it is bound to the NlaPro domain, preferably in cis, has NTPase activity (Mathur and Savithri, 2012). The NlaPro is the protease domain responsible for the proteolytic processing of the central and $\mathrm{C}$-terminal regions of the potyviral polyprotein (Adams et al., 2005). Nlb is the RNA-dependent RNA polymerase, or RNA replicase, responsible for potyviral genome replication (Hong and Hunt, 1996). $\mathrm{Nlb}$ could play be important for potyviral infection through the interaction with proteins that regulate diverse cellular processes in the nucleus of the infected cells (Xiong and Wang, 2013). Finally, the CP is the only structural protein. It is involved in the encapsidation of the viral RNA, but has also been implicated in genome amplification, movement, aphid transmission (Wylie et al., 2002) and in the regulation of viral RNA amplification (Bhat et al., 2013; Zhao et al., 2013)

\subsubsection{Ecology, epidemiology and disease control}

Potyviruses have a worldwide distribution and infect species of most angiosperm families. Most potyviruses infect dicotyledonous plants, with only a few of them infecting monocotyledonous hosts. Most virus species of the genus may cause a large range of macroscopic symptoms, which in many cases result in serious diseases of crops with high socio-economical relevance (e.g. Walsh and Jenner, 2002; Quenouille et al., 2013; Rybicki, 2015). In the Iberian Peninsula, potyviruses are important crop pathogens, causing severe losses in commercially important plant species of various botanical families (Melgarejo et al., 2004) such as: Asteraceae and Brassicaceae (Moreno et al., 2004a), 
Boraginaceae and Fabaceae (Luis-Arteaga et al., 1996), Cucurbitaceae (LuisArteaga et al., 1998; Moreno et al., 2004b), Poaceaceae (Achon and Sobrepere, 2001) or Solanaceae (Fereres et al., 1993; Navas-Castillo et al., 1999). In these hosts, potyviruses induce visible symptoms, often causing stunting, chlorotic vein banding, mosaic mottling, necrosis, and/or distortion of leaves. In monocotiledoneous hosts, potyviruses induce longitudinal chlorotic or necrotic streaks in the leaves. Flowers, seeds, and fruits are also affected by numerous potyviruses (Shukla et al., 1994). The nature and extent of symptoms for a specific host genotype depend on the particular virus strain, as well as on environmental conditions, probably through their influence on host physiology and development (Revers et al., 1999). There are examples of potyviruses involved in cases of synergism with other unrelated viruses, resulting in symptoms that are more severe than those induced by either virus alone (Pruss et al., 1997; Sikora et al., 1998).

Potyviruses are horizontally transmitted by aphids in a non-persistent manner and are transmissible experimentally by mechanical inoculation. Some viruses are also transmitted vertically through the seeds (King et al., 2012). Non-persistent virus transmission is characterized by very short acquisition and inoculation times of seconds to minutes. Thus, once an aphid has left a virusinfected source plant and begun feeding on an uninfected plant, its ability to transmit virus is short-lived ( $\mathrm{Ng}$ and Perry, 2004). Usually, a number of aphid species are capable of transmitting a particular potyvirus, and thus the level of vector specificity is relatively low. Hence, in a given host population potyvirus epidemiology is determined by the predominant aphid species and ecological and climatic conditions (Fereres et al., 1993; Sikora et al., 1998). The presence or absence of aphids may affect initial levels and distribution of potyviruses in a plant community, and subsequent vector activity will influence further distribution of the viruses within and between plant communities (Pleydell et al., 2018). Hence, in general, higher potyvirus prevalence will coincide with periods where vectors are abundant (Fereres et al., 1993; Katis et al., 2006; Chatzivassiliou et al., 2016). Other factors that may favor potyvirus epidemics are the emergence of more aggressive virus strains (Larsen et al., 2008; Desbiez et al., 2009), the traffic of infected plant material in a global market (García et al., 2014), or the introduction of new hosts in a geographical area 
that act as virus sources when in the proximities of susceptible crops (Hobbs et al., 2000).

Control of potyviral diseases is difficult. Insecticide sprays for aphids are used by farmers to control potyvirus spread. However, these are generally ineffective because of the rapid acquisition and inoculation periods required by aphid vectors, such that vectors transmit the virus before the insecticide acts. In some cases, insecticides can even speed up virus spread by increasing vector activity (Dedryver et al., 2010). Cross-protection using mild virus isolates that can protect plants against a severe isolate of the same virus, appears to be a more durable strategy for controlling potyvirus diseases (Yarden et al., 2000; Zhou, 2012). However, there are a number of limitations in cross-protection use, which include practical and safety concerns (Lecoq and Raccah, 2001). The most effective and simplest method of control of potyvirus diseases is through the use of host plant resistance. This has been a useful approach and has provided stable resistance to several potyviruses (Lecoq et al., 2004; Gómez et al., 2009). A less commonly used approach has been to breed crop cultivars with resistance to vectors, which has the potential added benefit of reducing losses associated with vector feeding damage (Dogimont et al., 2010). Methods of genetic engineering have been used to introduce genes for virus resistance obtained from novel sources. Some examples of this strategy include cultivated vegetables with coat protein-mediated resistance to several potyvirus species (Lindbo and Falk, 2017).

Besides the damage that potyviruses can cause in crops, some of them have been reported to infect weeds (e.g. dos Santos Martins et al., 2016; McLeish et al., 2017) and wild plant species (Malpica et al., 2006; Pagán et al., 2010), and symptoms induced by potyviruses in wild plant communities are not always obvious (Rodríguez-Nevado et al., 2017). Few studies have investigated potyvirus presence in wild ecosystems of the Iberian Peninsula, and such studies often focused in potyviruses known to be crop pathogens (McLeish et al., 2017, but see Rodríguez-Nevado et al., 2017). These studies indicate that, in wild ecosystems, potyviruses may be important ecological agents influencing the dynamics of the populations of their hosts. In addition, given that agricultural lands occupy more than a third of Spain territory, evergreen oak forests and other natural ecosystems are often adjacent to agroecosystems (see section 
1.4), which favours plant virus dispersal between both types of ecosystems (Alexander et al., 2014). Thus, potyviruses present in wild ecosystems may have the potential to cause epidemics in adjacent crops (McLeish et al., 2017; Rodríguez-Nevado et al., 2017). Despite this ecological and economic relevance, the factors affecting potyvirus infection risk and population genetic structure in wild ecosystems remain largely unexplored.

\subsubsection{Genetic diversity and evolution}

As previously stated, potyviruses comprise the largest and economically most important genus of plant RNA viruses. The genus Potyvirus characterized by a very large species and genetic diversity. Potyviruses are emerging in new geographic regions as potential epidemic pathogens (some recent examples in Rodríguez-Nevado et al., 2017 and Desbiez et al., 2017). For this reason, it is important to gain a better understanding of the origin, biogeographical diversification and evolution of potyviruses. Phylogenetic analyses based on the potyvirus CP gene for approximately half of all known potyvirus species indicated that the genus Potyvirus probably emerged from a virus of monocotyledonous plants about 6,600 years ago in Southwest Eurasia or North Africa, coinciding with the dawn of agriculture (Gibbs et al., 2008). Derived from the dating of the origin of potyviruses, it has been suggested that conditions generated by agriculture and a propitious climate enabled potyviruses and their vectors to spread, speciate and attain their current dominance (Gibbs et al., 2008).

Sequenced isolates has been obtained from experimental and natural populations in order to analyse how potyvirus populations generate genetic diversity. The evolutionary rate estimated for potyviruses is of $1.15 \times 10^{-4}$ nucleotide substitutions/site/year (Gibbs et al., 2008), a value within the range of RNA viruses of both plants and animals (Duffy et al., 2008). This estimate derives from a data set containing sequences from potyvirus species in which recombination has not been detected. It is unclear the relative importance of recombination for the generation of genetic variation in potyvirus populations. Although in general, mutation plays a central role (Tromas and Elena, 2010), several studies have shown that recombination events are especially common 
in some potyviruses (Chare and Holmes, 2006). Indeed, both intraspecies (Bousalem et al., 2003; Ohshima et al., 2002; Moreno et al., 2004b) and interspecies (Desbiez and Lecoq, 2004; Larsen et al., 2005; Gell et al., 2015) recombination events are involved in potyviral evolution. Experimental studies indicated that potyvirus populations are genetically stable and seem to evolve under strong purifying selection (Schneider and Roossinck, 2001). However, genetic drift may be important also in the evolution of potyvirus populations (French and Stenger, 2003; Hughes, 2009). Despite all this effort in characterizing the genetic diversity of potyviruses, very little is known about what ecological factors determine changes in genetic diversity of potyvirus populations and on the relationship of these changes with virus emergence (Rodríguez-Nevado et al., 2017). 
2. OBJECTIVES 
The main objective of this thesis is to analyse the ecological determinants of plant virus infection risk and population genetic diversity in wild ecosystems, using as a model the virus species of the genus Potyvirus present in two wild ecosystems of the Iberian Peninsula: riparian and evergreen oak forests. To achieve this general objective, the following specific objectives will be addressed:

1. Ecological characterization of evergreen oak and riparian forest locations in the central plateau of the Iberian Peninsula; and identification of Potyvirus species, and analysis of their host range and prevalence in these ecosystems.

2. Analysis of the ecological factors affecting genus-wide potyvirus prevalence in evergreen oak and riparian forests.

3. Analysis of the ecological factors affecting potyvirus species-specific prevalence according to their host range: specialist (narrow host range) vs. generalist (wide host range).

4. Study of the ecological and epidemiological factors affecting the population genetic diversity, and the speciation mode, of the potyviruses present in riparian and evergreen oak forests at the genus-wide (macroevolution) level.

5. Study of the ecological and epidemiological factors affecting the population genetic diversity of the potyviruses present in riparian and evergreen oak forests at the species-specific (microevolution) level, and according to their host range. 
3. MATERIALS AND METHODS 


\subsection{Plant sampling and data collection in wild ecosystems}

\subsubsection{Description of sampling locations}

Field samplings were done in two different wild ecosystems: evergreen oak forests and riparian forests. Five locations of evergreen oak forests and five locations of riparian forests were visited during 2013, 2014 and 2015. The locations were distributed in a transect of $300 \mathrm{~km}$ (north-south) in the center of the Iberian Peninsula encompassing the Duero and Tajo watersheds and the provinces of Valladolid, Segovia, Madrid, and Toledo. The sampled locations are listed in Table $\mathbf{3 . 1}$ and their location in the Iberian Peninsula is represented in Figure 3.1.

Table 3.1. Locations of lberian evergreen oak forests and riparian forests visited during 2013 , 2014 and 2015 in this thesis.

\begin{tabular}{|c|c|c|c|c|c|}
\hline Ecosystem & Location $^{1}$ & Province & $\begin{array}{l}\text { Latitude } \\
\text { (№) }\end{array}$ & $\begin{array}{l}\text { Longitude } \\
\text { (Wo) }\end{array}$ & $\begin{array}{l}\text { Elevation } \\
(\mathrm{m})\end{array}$ \\
\hline $\begin{array}{l}\text { Evergreen oak } \\
\text { forest }\end{array}$ & $\begin{array}{c}\text { CARBONERO } \\
\text { EL MAYOR (CEM) }\end{array}$ & Segovia & 41.148 & -4.318 & 928 \\
\hline $\begin{array}{l}\text { Evergreen oak } \\
\text { forest }\end{array}$ & EL PARDO (PAR) & Madrid & 40.501 & -3.740 & 616 \\
\hline $\begin{array}{l}\text { Evergreen oak } \\
\text { forest }\end{array}$ & MONTEGANCEDO (MON) & Madrid & 40.403 & -3.836 & 713 \\
\hline $\begin{array}{l}\text { Evergreen oak } \\
\text { forest }\end{array}$ & CENICIENTOS (CEN) & Madrid & 40.259 & -4.449 & 773 \\
\hline $\begin{array}{l}\text { Evergreen oak } \\
\text { forest }\end{array}$ & MARJALIZA (MAR) & Toledo & 39.578 & -3.922 & 853 \\
\hline Riparian forest & POLLOS (POL) & Valladolid & 41.444 & -5.148 & 679 \\
\hline Riparian forest & PALAZUELOS (PAL) & Segovia & 40.921 & -4.052 & 1083 \\
\hline Riparian forest & RASCAFRÍA (RAS) & Madrid & 40.890 & -3.884 & 1162 \\
\hline Riparian forest & EL ESCORIAL (ESC) & Madrid & 40.574 & -4.153 & 913 \\
\hline Riparian forest & $\begin{array}{c}\text { SANTA CRUZ } \\
\text { DE RETAMAR (SCR) }\end{array}$ & Toledo & 40.279 & -4.231 & 589 \\
\hline
\end{tabular}

${ }^{1}$ Name of the nearest town and three-letter code (in parenthesis) indicating the name of the sampled locations.

The evergreen oak forest locations were chosen to be representative of this ecosystem. That is, to include natural woodland areas dominated by trees and shrubs and with clearings where herbaceous species are abundant. The riparian forest locations were chosen to be closer than three meters from the nearest riverside. The predominant tree species on the riverside was not considered a criterion for selecting these locations. Locations in this study were selected such that there was no evidence of human management. 


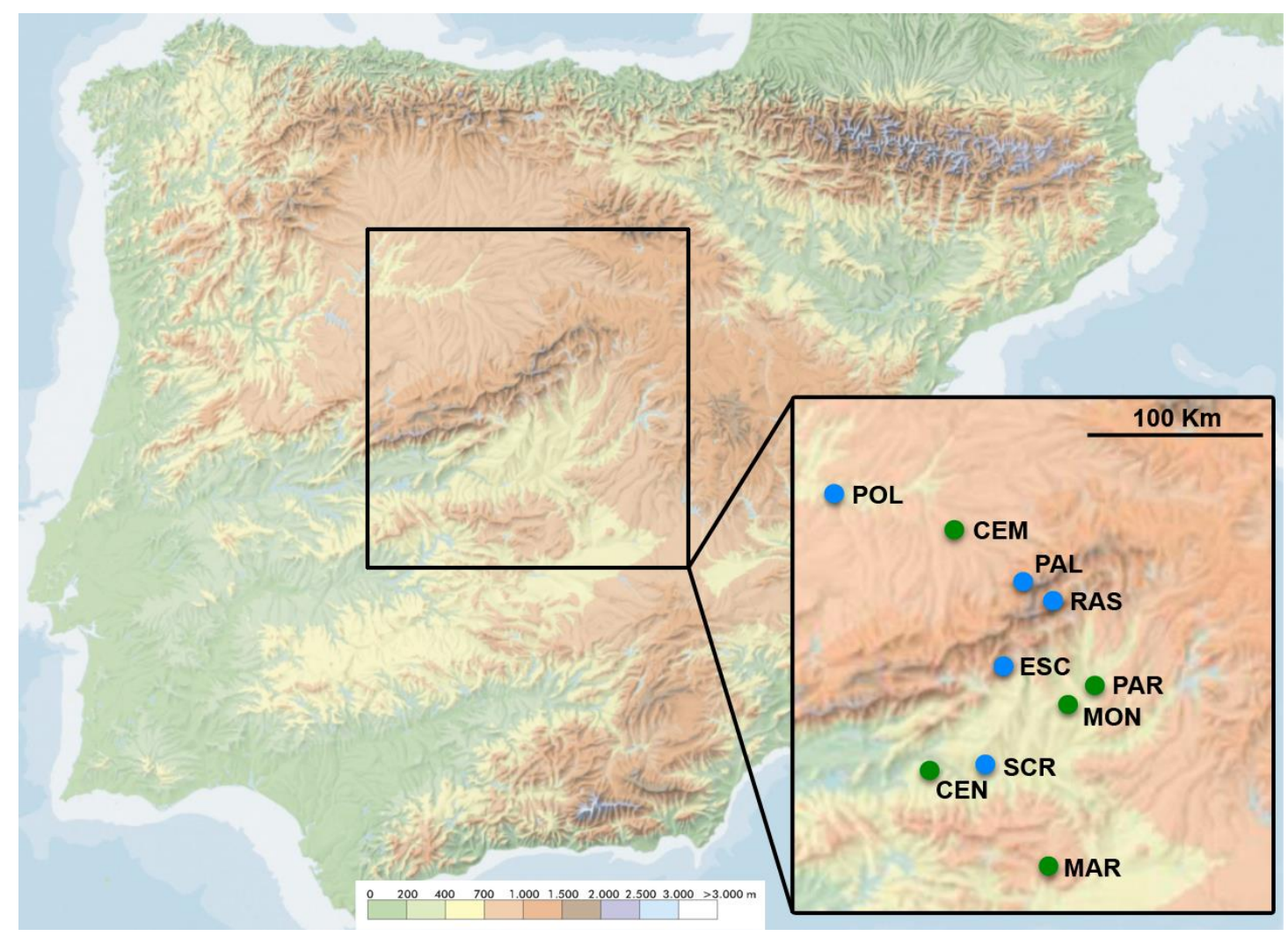

Figure 3.1. Geographic distribution of the evergreen oak (green dots) and the riparian forest (blue dots) locations visited in this study. Color scale indicates the elevation gradient. Abbreviations for each location are as in Table 3.1.

\subsubsection{Plant sampling design}

At each location, a plot of $25 \times 25$ meters was defined, and within this plot a random-systematic sampling was performed. This sampling design consisted on selecting a random starting point in the plot and then collecting samples in a fixed, periodic interval called sampling interval (Barbour et al., 1999). In this work, the $25 \times 25$ plot was divided in a grid of $1 \times 3$ meters (Figure 3.2). At each of these rectangles or sampling intervals, plant material from the most abundant species was harvested. If the most abundant species was a tree or a shrub, at least three leaves from different branches of one individual of such species was collected. If the most abundant species was herbaceous, the stem and leaves of an individual were harvested. A total of 200 samples were collected at each location. Samplings were performed during the months of spring (April and May), summer (June and July) and autumn (October and November) to account 
for seasonal differences in plant species composition and phenological stage, and in aphid activity. A total of 12,000 samples were collected in these surveys.

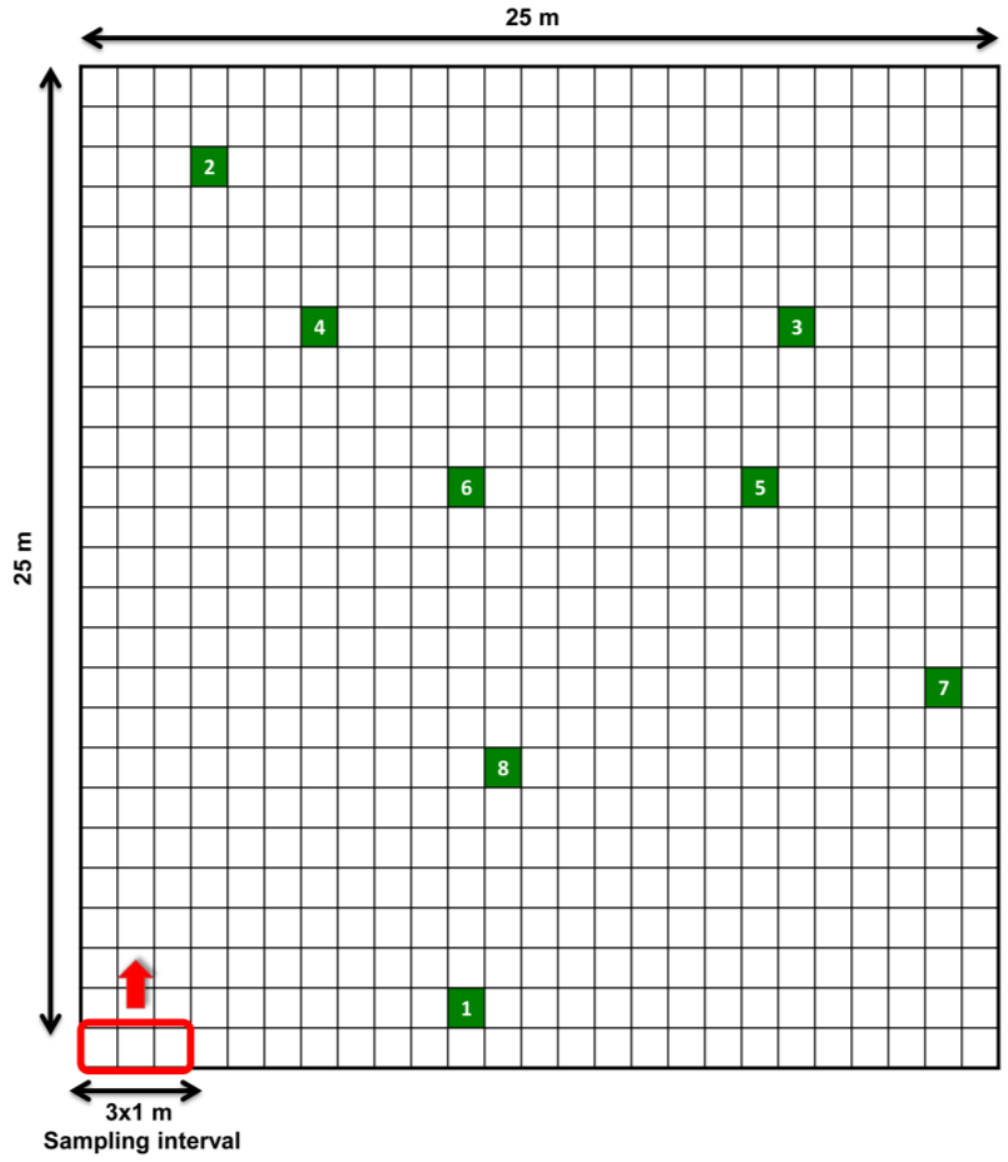

Figure 3.2. Sampling design performed in the monitored locations. A plot of $25 \times 25$ meters divided in a grid of $1 \mathrm{~m}^{2}$. Red rectangle indicates the spatial unit of sampling ( $3 \times 1 \mathrm{~m}$ interval), and the arrow indicates sampling direction. Green squares represent the eight quadrants of $1 \mathrm{~m}^{2}$ randomly selected for quantification of plant biomass (see section 3.1.3).

\subsubsection{Monitoring and estimation of ecological factors}

At each visit, the following information was collected in the five evergreen oak and the five riparian forests locations:

1) Plant biodiversity: In parallel with plant sampling, additional individuals representative of each collected plant species were harvested and inventoried in herbariums. These herbariums were used to identify the botanical family and species of each collected individual. Plant identification was performed by Professor Rosario G. Gavilán (Plant Biology Department, Universidad Complutense de Madrid, Madrid, Spain). This allowed determining the number 
of species, and the number of individuals per species collected at each location and visit. Using this data, plant species richness and various diversity indexes that consider both species richness and evenness were calculated as measurements of plant species diversity, which was used as a proxy of plant biodiversity:

i) Species richness (S): Is the simplest way of measuring plant biodiversity, and it is defined as the number of plant species present in an ecosystem/location.

ii) Shannon's diversity index $\left(H^{\prime}\right)$ : This diversity index provides more information about plant community composition than $S$, as it reflects the heterogeneity of a community based on two factors: the number of species present and their relative abundance (Shannon, 1948). In order to obtain the Shannon's index, the relative abundance of each species $\left(p_{i}\right)$ is calculated, and then multiplied by its natural logarithm $\left(\ln p_{i}\right)$. The resulting product is summed across species (S), and multiplied by -1 . The higher the value of $\mathrm{H}^{\prime}$, the greater the diversity:

$$
\mathrm{H}^{\prime}=-\sum_{i=1}^{n} p_{i} \ln p_{i}
$$

In this thesis, the relative abundance of a given plant species $\left(p_{i}\right)$ was estimated as the number of individuals of the species $i\left(n_{i}\right)$ relative to the total number of individuals collected at each location and visit $(n=200)$ :

$$
p_{i}=n_{i} / n
$$

iii) Shannon's equitability index $\left(E_{H}\right)$ : This is a modification of $\mathrm{H}^{\prime}$ that allows exploring whether all the species have similar relative abundances or some of them exhibit pronounced dominance in the ecosystem/location. It is calculated by dividing $\mathrm{H}^{\prime}$ by $\mathrm{H}^{\prime} \max$ (here, $\mathrm{H}^{\prime} \max =\mathrm{lnS}$ ). Equitability acquires a value between 0 (one species is dominant) and 1 (abundance evenness between species). 
2) Life Cycle: Plants were classified according to their life cycle in two categories: perennial and annual plants. This information was obtained from Real Jardín Botánico (Consejo Superior de Investigaciones Científicas, CSIC) (http://www.floraiberica.es/).

3) Plant biomass: At each location and visit, eight quadrants of $1 \mathrm{~m}^{2}$ were randomly selected and photographed (Figures 3.2 and 3.3), and these photographs were used to calculate the plant coverage using Image $\mathrm{J}$ v1.5 (Schneider et al., 2012). Briefly, the green fraction of each photograph was selected using the "Threshold Color" option, and considered to be the area occupied by living plants. This provided an estimate of the vegetation coverage of each quadrant. Also, in the eight quadrats the maximum height of living plants was measured at three points across the diagonal of the quadrant (Figure 3.3). Values of plant coverage and height were used to calculate the apparent biovolume $(\mathrm{V})$, which is the most common measure for aerial biomass (Castro et al., 1996). The plant biovolume at each quadrat was calculated as the mean of the three plant height measures multiplied by the vegetation coverage. Plant biovolume in the $25 \times 25 \mathrm{~m}$ plot $\left(\mathrm{m}^{3}\right)$ was estimated by averaging values in the eight quadrants at each location and visit.
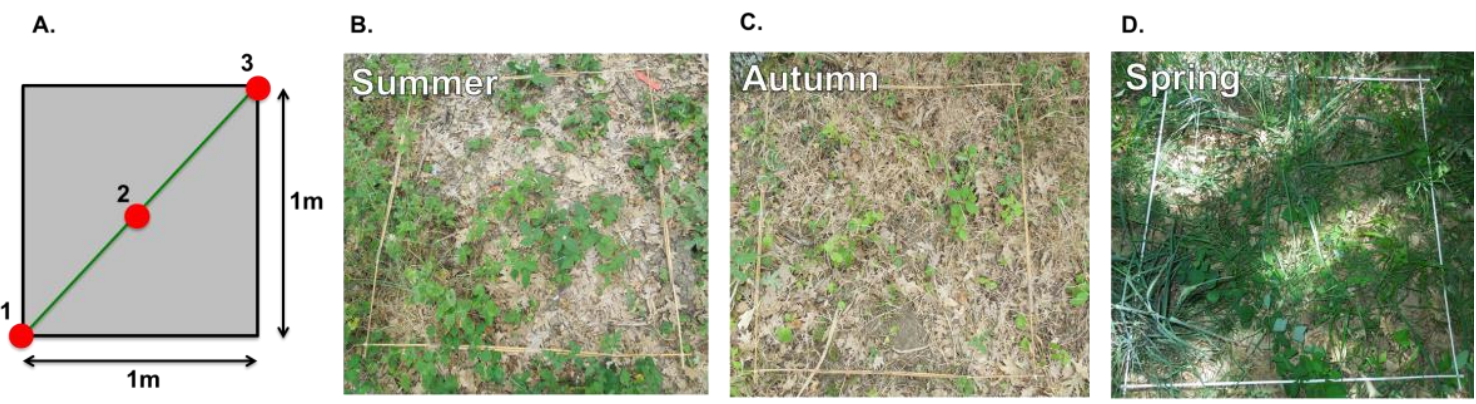

Figure 3.3. Schematic representation and examples of $1 \mathrm{~m}^{2}$ quadrants used to quantify plant biomass. (A) Red dots across the diagonal of the grey square indicate the three points in which plant height was measured. ( $B$ to $D$ ) Photographs of $1 \mathrm{~m}^{2}$ quadrants taken in the same location at different seasons.

4) Plant Density: This trait was estimated for each species using distance methods. These methods can be used to determine whether plants of the same 
species are distributed randomly, regularly, or are clumped (Barbour et al., 1999). In this work, the Mean Euclidean nearest neighbor distance (ENN_MN) was used. ENN_MN was estimated using the spatial pattern analysis program Fragstats v4.2.1 (McGarigal et al., 2012). The ENN_MN is defined using simple Euclidean geometry as the mean of the shortest straight-line distance between an individual of a plant species and its nearest neighbor of the same species. The larger this distance, the lower the plant density. This index could be estimated because the sampling scheme described above allowed identifying the position of each sampled plant within the $25 \times 25 \mathrm{~m}$ plot.

5) Climatic variables: Data related with temperature $\left({ }^{\circ} \mathrm{C}\right)$, relative humidity $(\%)$ and rainfalls $(\mathrm{mm})$ at the months in which locations were visited were retrieved from the closest meteorological stations to each location, which were at a similar altitude (Table 3.2). This data was provided by the Agencia Estatal de Meteorología (AEMET, Spain, http://www.aemet.es).

Table 3.2. Meteorological stations from which climatic variables of evergreen oak and riparian forests locations were obtained.

\begin{tabular}{|c|c|c|c|c|c|c|}
\hline Ecosystem & Location & $\begin{array}{l}\text { Meteorological } \\
\text { station }\end{array}$ & $\begin{array}{c}\text { Indicative } \\
\text { code }^{1}\end{array}$ & $\begin{array}{l}\text { Latitude } \\
\text { (№) }\end{array}$ & $\begin{array}{l}\text { Longitude } \\
\text { (Wo) }\end{array}$ & $\begin{array}{l}\text { Elevation } \\
(\mathrm{m})\end{array}$ \\
\hline $\begin{array}{l}\text { Evergreen oak } \\
\text { forest }\end{array}$ & $\begin{array}{l}\text { CARBONERO } \\
\text { EL MAYOR }\end{array}$ & Miguelañez & 2482B & 41.073 & -4.223 & 870 \\
\hline $\begin{array}{l}\text { Evergreen oak } \\
\text { forest }\end{array}$ & EL PARDO & $\begin{array}{c}\text { Ciudad } \\
\text { Universitaria }\end{array}$ & $3194 U$ & 40.27 & -3.432 & 664 \\
\hline $\begin{array}{l}\text { Evergreen oak } \\
\text { forest }\end{array}$ & MONTEGANCEDO & $\begin{array}{l}\text { Pozuelo } \\
\text { de Alarcón }\end{array}$ & $3194 Y$ & 40.265 & -3.484 & 665 \\
\hline $\begin{array}{l}\text { Evergreen oak } \\
\text { forest }\end{array}$ & CENICIENTOS & $\begin{array}{l}\text { Rozas de } \\
\text { Puerto Real }\end{array}$ & $3330 Y$ & 40.184 & -4.291 & 890 \\
\hline $\begin{array}{l}\text { Evergreen oak } \\
\text { forest }\end{array}$ & MARJALIZA & $\begin{array}{l}\text { Mora de } \\
\text { Toledo }\end{array}$ & $3254 \mathrm{Y}$ & 39.411 & -3.465 & 717 \\
\hline Riparian forest & POLLOS & Rueda & $2507 Y$ & 41.252 & -4.575 & 715 \\
\hline Riparian forest & PALAZUELOS & Segovia & 2465 & 40.564 & -4.073 & 1005 \\
\hline Riparian forest & RASCAFRÍA & El Paular & $3104 Y$ & 40.532 & -3.531 & 1159 \\
\hline Riparian forest & EL ESCORIAL & Alpedrete & $3268 \mathrm{C}$ & 40.393 & -4.010 & 924 \\
\hline Riparian forest & $\begin{array}{l}\text { SANTA CRUZ } \\
\text { DEL RETAMAR }\end{array}$ & $\begin{array}{l}\text { Castillo } \\
\text { Bayuela }\end{array}$ & $3362 Y$ & 40.062 & -4.405 & 560 \\
\hline
\end{tabular}

${ }^{1}$ Indicative code of the meteorological station according to AEMET.

\section{6) Edaphic factors:}

(i) Soil $\mathrm{pH}$ : at each location, soil $\mathrm{pH}$ was obtained from the "European Soil Data Center" (http://eusoils.jrc.ec.europa.eu/library/data/pH/). These values 
were confirmed in soil samples collected at each location by determining the $\mathrm{pH}$ according to the protocol described by Rojas (1989).

(ii) Soil moisture: a soil moisture probe (TDR 100; Spectrum Tech Inc., Plainfield, IL, USA) was used to determine volumetric water content (VWC) of the soil to $12 \mathrm{~cm}$ deep. Measures were taken at the eight quadrants of $1 \mathrm{~m}^{2}$ randomly selected in each location. Four measurements were taken around each of the quadrants (one at each quadrat side) and data was averaged.

A summary of the value of each of the parameters recorded at each location and visit is provided in Appendix 1, Table A1.1 and A1.2.

\subsubsection{Analysis of the sampling effort}

The species richness captured by a given sampling scheme (sampling effort) can be visualized as the asymptote of an accumulation curve that represents the observed species richness relative to the number of sampled individuals. Once this asymptote is reached, additional sampling will not increase species richness (Gotelli and Colwell, 2010). The effectiveness of the sampling effort to represent the total species richness in the monitored locations of each ecosystem was analysed using these accumulation curves.

The number of individuals of each sampled species identified at each location and season during the monitored period was used to calculate the mean number of species and its standard deviation per sampling effort unit with the EstimateS 9.1 software (Colwell, 2013). The potential effect of the order in which the samples were collected was removed by randomizing 100 times their order in each dataset. Accumulation curves were plotted for each location of evergreen oak and riparian forests in each monitored season, and the asymptote values of the accumulation curves were compared with values of nonparametric estimators of species richness, which use information on the rare species in a sample to estimate the number of undetected species (Gotelli and Chao, 2013). The estimators used were the Chao 1 estimator, which accounts for the number of species that are represented only by one or two individuals, and the Abundance-based Coverage Estimator (ACE) that is based on the observed number of common and infrequent species (considering as infrequent 
those species with 10 or fewer individuals). Because both estimators led to the same values, only results derived from the Chao 1 estimator are shown. ACE and Chao 1 richness estimators were computed along with log-linear 95\% confidence intervals (Cls) (Chao, 1987). The Cls were used to estimate the precision of the richness estimates and to infer the statistical significance between mean observed and estimated species richness: evidence of statistical difference was considered when the observed mean species richness lied outside the $95 \% \mathrm{Cls}$ of the estimated mean species richness (Schenker and Gentleman, 2001; Ramsey and Schafer, 2002).

\subsection{Potyvirus detection and identification}

\subsubsection{RNA extraction from plant samples}

Infection by potyvirus species was detected in total RNA preparations from plant material. The Cetyltrimethylammonium (CTAB) - Polyvinyl pyrrolidone (PVP) method (Chang et al., 1993) was selected for purification of total plant RNA. Since salt precipitation prevented efficient RNA purification of some plant species, the method was modified adjusting the amount of $\mathrm{NaCl}(5 \mathrm{M})$ to $50 \mu \mathrm{l}$ per $500 \mu \mathrm{l}$ of supernatant, instead of $100 \mu \mathrm{l}$, during the final precipitation step with 2.5 volumes of cold absolute ethanol $\left(-20^{\circ} \mathrm{C}\right)$. This method allowed efficient and consistent RNA purification from the majority of the plant species present in the studied ecosystems, including problematic tissues harboring large amounts of polysaccharides, polyphenols and other secondary metabolites.

The RNA integrity was verified after running an aliquot of the purified RNA in a denaturing $1.2 \%$ agarose gel, stained with ethidium bromide (EtBr), in Trisacetate-EDTA (TAE) buffer. The RNA extraction yield and the presence of contaminants was determined by measuring absorbance $(A)$ of the intact total RNA extracts at $230 \mathrm{~nm}, 260 \mathrm{~nm}$ and $280 \mathrm{~nm}$ in a NanoDrop ${ }^{\circledR}$ ND-1000 spectrophotometer (Thermo Scientific, Waltham, MA, USA). Samples with yield greater than 10ng/ $\mu$ (in a total volume of $30 \mu \mathrm{l}$ ), and A260/A280 and A260/A230 ratios between 1.7-2.0 and $\sim 2.0$, respectively, were considered to have good quantity and quality for further analysis. 


\subsubsection{Detection and identification of virus species of the genus Potyvirus}

The presence of virus species within the genus Potyvirus was analysed by Onestep SYBR Green-based real-time RT-PCR in the LightCycler® 480 Real-Time PCR System (Roche Diagnostics, Mannheim, Germany). For each run, $10 \mathrm{ng}$ of total plant RNA were added to the Brilliant III SYBR® Green Ultra-Fast QRTPCR Master Mix (Agilent Technologies, Santa Clara, CA, USA) following the manufacturer's recommendations. Universal primers NIb2F and NIb3R (Zheng et al., 2010) for species of the genus Potyvirus were used to amplify a region of $\sim 350$ nucleotides of the gene that encodes the NIb protein. The thermal profile consisted of a 5 -min pre-incubation step at $65^{\circ} \mathrm{C}, 10$-min RT step at $50^{\circ} \mathrm{C}$ and 5 $s$ of Taq polymerase activation at $95^{\circ} \mathrm{C}$, followed by 50 cycles of PCR at $95^{\circ} \mathrm{C}$ for $10 \mathrm{~s}$ (denaturation), $50^{\circ} \mathrm{C}$ for $20 \mathrm{~s}$ (annealing), and $72^{\circ} \mathrm{C}$ for $30 \mathrm{~s}$ (extension). Following amplification, a melting curve analysis, which determines the specific melting temperature $\left(T_{m}\right)$ of an amplicon, was performed to verify that the obtained amplicons were of the expected size. This analysis consisted in a denaturation step at $95^{\circ} \mathrm{C}$ for $30 \mathrm{~s}$, lowered to $60^{\circ} \mathrm{C}$ for $1 \mathrm{~min}$, and followed by an incubation step in which the temperature was increased to $95^{\circ} \mathrm{C}$ at a rate of $0.11^{\circ} \mathrm{C} / \mathrm{s}$ with continuous reading of fluorescence. $T_{m}$ values were analyzed using the $T_{m}$ Calling analysis module in LightCycler ${ }^{\circledR} 480$ Software Version 1.5.

In all real-time RT-PCR analyses, RNA purified from Turnip mosaic virus isolate UK 1 (UK 1-TuMV), a well-characterized potyvirus species (Sánchez et al., 2003), was included as positive control. For that, UK 1-TuMV, kindly provided by Prof. Fernando Ponz (CBGP UPM-INIA, Spain), was propagated in Indian mustard [Brassica juncea (L.) Czern.] and viral particles were purified according to the protocol described in Sánchez et al., 1998. Viral genomic RNA was extracted from purified virions by $1 \%$ SDS treatment and phenol-chloroform extraction with ethanol precipitation. Viral RNA was re-suspended in DEPCwater and kept at $-80^{\circ} \mathrm{C}$. Real-time RT-PCR negative controls included the reagent mix without RNA template. Real-time RT-PCR amplification was confirmed in $1.2 \%$ agarose gel. PCR products of the expected size were considered as positive detections of potyviruses. To confirm potyvirus presence, these products were purified using the StrataPrep DNA Gel Extraction Kit 
(Agilent Technologies) and sequenced. Sequences were assembled using MEGA 6 (Tamura et al., 2013), and their percentage of nucleotide identity with the potyviral sequences available in GenBank was analysed using BLASTN (http://blast.ncbi.nlm.nih.gov/Blast.cgi). Following the ICTV criteria (King et al., 2012), 350-nt Nlb sequences with a nucleotide identity over 55\% with any known species of the genus Potyvirus were considered as belonging to this genus, and sequences with nucleotide identity between $55 \%$ and $76 \%$ were considered as belonging to a non-previously described species of this genus (Adams et al., 2005).

It should be noted that, according to King et al., 2012, the demarcation criteria for potyvirus species is based upon the coat protein gene. However, in this thesis a fragment of the Nlb was used as potyvirus species demarcation criterion. Thus, whether the Nlb region of $\sim 350$ nucleotides contained sufficient phylogenetic information to identify different potyvirus species with the same accuracy than the CP gene was analysed. To do so, sequences of potyvirus isolates for which the 350-nt Nlb fragment and the full-length CP genes were available ( $n=271$ ) were retrieved from GenBank. These NIb and CP sequences were aligned and phylogenetic trees were constructed for each genomic region as described in section 3.2.3. Congruence between the phylogenies obtained with the NIb and the CP sequences was tested with CopyCat v.2.03 (MeierKolthoff et al., 2007). A total of 5 (2\%) non-significant and 266 (98\%) significant associations between phylogenies of the 350-nt Nlb fragment and the CP sequences of potyvirus isolates were retrieved in the cophylogenetic analysis using a significance threshold of $P=0.050$. Thus, results indicated that the topology of the tree obtained with the 350nt region of the Nlb gene was congruent with that obtained with the CP sequence (Appendix 1, Table A1.3). Hence, both genomic regions allowed identifying Potyvirus species with comparable accuracy.

\subsubsection{Phylogenetic characterization of potyvirus species}

Phylogenetic relationships between the potyvirus species detected in evergreen oak and riparian forests and the other members of the genus Potyvirus were inferred using Maximum Likelihood methods. To perform this analysis, Nlb 
sequences of the potyviruses detected in this thesis were aligned with those of representative isolates of all known potyviruses. Sequences were aligned using the MAFFT algorithm (Katoh et al., 2002) and the alignment was refined using MUSCLE (Edgar, 2004), both integrated in GENEIOUS v.11 software (Kearse et al., 2012). Using this alignment, a phylogenetic tree was constructed using PhyML package v3.0 (Guindon et al., 2010) under the Tamura-Nei model of nucleotide substitution, with a Gamma distribution of substitution rates, as this was selected as the best-fitted nucleotide substitution model in jModelTest v. 2.1.10 (Darriba et al., 2012). The Nearest-Neighbor Interchanges (NNI) and Subtree Pruning and Regrafting (SPR) heuristic methods were used to estimate tree topology, and the best topology obtained was selected. The support for each internal branch of the phylogeny was estimated by the $\mathrm{SH}$-like approach. This is a faster but equally accurate method than the bootstrap methods, and is recommended when a large number of sequences is analysed (Anisimova et al., 2011). The tree was visualized and edited in FigTree software v1.4 (http://tree.bio.ed.ac.uk/software/figtree/).

Similar phylogenetic analyses were done using the CP gene. To do so, the $\mathrm{CP}$ gene sequence of each isolate was also obtained and used in phylogenetic analyses. For that, universal potyvirus primer NlbFor1 ( $\mathrm{Ha}$ et al., 2008) and an Oligo(dT) ${ }_{18}$ Primer (Life Technologies) were used to obtain the 3 'end (900nt) of these isolates, and these fragments were sequenced as described above. Because NlbFor1 is a universal primer for Potyvirus species, specific primers for the CP of the three most prevalent virus species detected in this work were designed for some of the analyses presented in this thesis (see Results section). For that, the 900nt sequences were aligned as described above and scanned for conserved regions in order to design CP speciesspecific primers. The suitability of the designed primers for species-specific CP amplification was analyzed using OligoAnalyzer® Tool (http://eu.idtdna.com/calc/analyzer). Amplifications were done by Reverse Transcription Polymerase Chain Reaction (RT-PCR). AffinityScript Multiple Temperature Reverse Transcriptase (Agilent Technologies) was used to produce the cDNA, which was used as a template for DNA amplification using the Biotools DNA Polymerase (Biotools, B\&M Labs,S.A., Madrid, Spain) (Table 3.3). Unfortunately, not all the CP sequences of isolates for which the 350-nt 
$\mathrm{Nlb}$ fragment was available could be obtained. Thus, in the Results section analyses with both the $\mathrm{Nlb}$ and the $\mathrm{CP}$ gene sequences are presented.

Table 3.3. Sequence of species-specific primers used to amplify the CP gene of the isolates of the most prevalent potyviruses obtained in this thesis.

\begin{tabular}{lll}
\hline Primer & Primer Sequences 5'-3' & Potyvirus species \\
\hline WHMV-CPFor & AGAGAAACTGGTGACAGTCCTGAGTT & Wild hop mosaic virus \\
WHMVV-CPRev & CTGGCAAGACTCATGTCAGTCAAATTTCTC & \\
\hline MeRV-CPFor & CCAAAGCTTGAACAAGAGAGAATTGTTTCG & Mediterranean ruda virus \\
MeRV-CPRev & ACACCAAGCATGKTRTGCATAT & \\
\hline CIYVV-CPFor & GATGACAGCCARATGAAATT & Clover yellow vein virus \\
CIYVV-CPRev & GGAGAATTTAAAGACGGATACTCTA & \\
\hline
\end{tabular}

\subsection{Comparative genomics of potyviruses}

\subsubsection{Detection of recombination in potyvirus populations}

Inter and intra-species recombination events in the CP gene and in the partial $\mathrm{Nlb}$ gene sequences were analyzed. These analyses were done using the sequences from potyvirus isolates obtained in this thesis (Appendix 1, Table A1.4), and also including reference sequences of these genes from all known potyviruses (Appendix 1, Table A1.5). Recombination was detected utilizing four different methods based on different assumptions (Posada, 2002) as implemented in the RDP4 package (http://darwin.uvigo.es/rdp/rdp.html): RDP, GENECONV, Bootscan, and Chimaera, and employing the default parameters and a Bonferroni correction P-value cut-off of 0.05 (Martin et al., 2015). Only recombination signals detected by all methods were considered as positive to minimize false positives. With this criterion, no recombinants were detected. Analyses using the more relaxed criterion of considering as recombinants those detected by two or more methods to minimize false negatives yielded the same result.

\subsubsection{Analysis of nucleotide substitution rates}

\subsubsection{Robustness of temporal signal}

To test the strength of the temporal signal in these data, essential to the accurate estimation of substitution rates, the BEAST analyses described in 
section 3.3.2.2 were repeated on data sets in which sampling times were randomized such that they lack any temporal structure. Runs for randomized data were repeated 10 times. The mean and 95\% HPDs of the substitution rate estimates for the randomized data were then compared with those obtained from the real data; major differences in these estimates indicate the presence of temporal structure.

\subsubsection{Estimation of substitution rates}

For the partial NIb and the CP data set, rates of nucleotide substitution per site were estimated using the Bayesian Markov Cain Monte Carlo (MCMC) method available in the BEAST v1.8.3 package (Drummond and Rambaut, 2007). The best-fit model of nucleotide substitution in each case was determined using jModelTest v. 2.1.10 (Darriba et al., 2012), and all data sets were subsequently run using the general time-reversible substitution model with invariant sites and a gamma distribution of among-site rate variation $\left(\mathrm{GTR}+\mathrm{I}+\Gamma_{4}\right)$. These sequence data were analyzed using a relaxed (uncorrelated, lognormal) molecular clock (see Drummond et al., 2006) and a Bayesian skyline model as a coalescent prior, as estimating demographic parameters was not the aim of this study. In all cases, the BEAST analyses were run until all relevant parameters converged, with $10 \%$ of the MCMC chains discarded as burn-in. Statistical confidence is represented by values for the $95 \%$ highest probability density (HPD).

\subsubsection{Analysis of potyvirus population genetic diversity}

The different parameters of potyvirus population genetic diversity were estimated based on the CP and partial NIb gene sequences. Virus population genetic diversity $(\pi)$, as average pairwise nucleotide difference between sequences, and the average pairwise mean number of non-synonymous $\left(d_{N}\right)$ and synonymous $\left(d_{S}\right)$ nucleotide substitutions per site were estimated using MEGA 6 (Tamura et al., 2013) and the best-fitted nucleotide substitution model as determined by jModelTest v. 2.1.10 (section 3.2.3) (Darriba et al., 2012). Standard errors of each measure were based on 1,000 bootstrap replicates. $d_{N}$ and $d_{s}$ were also estimated by averaging values at individual codons using different methods implemented in the HYPHY program v.2.2 (SLAC, Single 
Likelihood Ancestor Counting; FEL, Fixed Effects Likelihood; IFEL, Internal Fixed Effects Likelihood; REL, Random Effects Likelihood; FUBAR, Fast Unbiased Bayesian Approximation) (Kosakovsky et al., 2005; Murrell et al., 2013). Selection pressures were estimated as the $d_{N} / d_{S}$ ratio. As comparable results were obtained with all these methods, only results of analyses using average pairwise values are shown. Finally, in each potyvirus population the number of haplotypes $(H)$ and the haplotype diversity $\left[H_{d}=\left(1-\Sigma x_{i}{ }^{2}\right) n /(n-1)\right.$, where $x_{i}$ is the frequency of an haplotype and $n$ is the population sample size (Nei and Tajima, 1981)] were calculated using DnaSP v.5 (Librado and Rozas, 2009).

\subsubsection{Analysis of the genetic structure of potyvirus populations}

Nlb- and CP-based bayesian trees were constructed using the Tamura-Nei substitution model with a Gamma distribution of nucleotide substitution rates. All analyses were run until relevant parameters converged, with $25 \%$ of the MCMC chains discarded as burn-in. These trees were constructed using MrBayes v3.2.6 (Ronquist et al., 2012). Maximum clade credibility (MCC) trees, with Bayesian posterior probability values providing a measure of the robustness of each node, were also summarized from the MrBayes tree samples using TreeAnnotator v1.8.3 available as part of the BEAST package (Drummond and Rambaut, 2007).

Association between potyvirus population geographic origin and host species, and the position of each virus isolate in the Nlb and CP-based phylogenies was assessed using the Association Index (Al), the Parsimony Score (PS) and the Monophyletic Clade Size (MC) statistics. PS and AI measure the degree to which sequences from virus isolates that share the same geographic origin/host species cluster together across the entire tree topology. MC assesses the same association but for each specific geographic origin/host species. Statistical significance of these indexes was obtaining by calculating empirical distributions of the three association statistics from the credible sample (posterior distribution) of Nlb-based phylogenetic trees constructed utilizing Bayesian Markov Chain Monte Carlo (MCMC) methods. Empirical distribution for each index, constructed with at least 2,000 trees, was compared with that resulting from randomizing 1,000 times the position of each 
sequence in the Bayesian tree. The null hypothesis of random phylogeny-trait associations was rejected at a significance level of $P=0.05$. This analysis was performed using the BaTS software (Parker et al., 2008).

\subsection{Biological and molecular characterization of a novel potyvirus in a wild ecosystem}

\subsubsection{Biological characterization of Mediterranean ruda virus (MeRV)}

For the characterization of MeRV described below, tissue of one mountain rue plant sampled in El Pardo and infected with MeRV was selected. This plant tested negative for other species of the genus Potyvirus by RT-PCR. More importantly, analyses of the NGS sequence data obtained from total RNA extracts of this field-infected mountain rue plant did not detect any other viral sequence. Tissue of the infected field plant was grinded in $0.1 \mathrm{M}$ phosphate buffer $\mathrm{pH} 7,0.02 \%$ sodium diethyldi-thiocarbamate (DIECA), and used to inoculate Nicotiana benthamiana plants. After 20 days, N. benthamiana plants developed a systemic mosaic. MeRV virions were purified from these plants according to the protocol described by Sánchez et al., 2013. Agarose gel electrophoresis of the virion preparation showed a single band, indicating that all virions in the preparation had the same size. Indeed, agarose gel electrophoresis of nucleic acid extracts from these virions showed a single band of ssRNA of about 9,000-10,000 nt. The same Nlb nucleotide sequence amplified from the field-infected mountain rue plant was obtained from virion preparations using universal potyvirus primers. Together, these results indicated that a single virus species was transferred from mountain rue to $N$. benthamiana plants, and that this species belonged to the genus Potyvirus, discarding the possibility of mixed viral infection. This isolate was named MeRVParP17, and its virions were used for further characterization.

To determine the host range and the symptoms induced by MeRV infection, different plant species of the botanical families Amaranthaceae, Asteraceae, Boraginaceae, Chenopodiaceae, Cistaceae, Cucurbitaceae, Fabaceae, Fagaceae, Poaceae, Rutaceae and Solanaceae were used (Table 
3.4). These species were chosen to include the most abundant species in the sampled evergreen oak forests (the ecosystem from which the virus was isolated), and the most common crops in the surrounding areas. For each plant species, four to seven plants (10-15 days old) were mechanically inoculated by applying purified virion RNA (100 ng/ul) in $0.1 \mathrm{M} \mathrm{Na}_{2} \mathrm{HPO}_{4}$ onto the first two completely expanded leaves dusted with carborundum. The inoculated plants were maintained in a greenhouse $\left(20-25^{\circ} \mathrm{C}\right.$, and $16 \mathrm{~h}$ of light), and symptoms were recorded over an 8-week period. Tissue from systemically infected leaves of symptomatic and asymptomatic plants was collected 20 days post-inoculation and tested for potyvirus infection by real-time RT-PCR using specific primers qCPFor (5'-GACTGACTATAGTTTAGCGCGC-3') and qCPRev (5'GCCTCTGATAGCTGCTGCTTTC-3') that amplify a 111-bp region of the MeRV CP gene.

Table 3.4. Plant families and species used to analyse MeRV host range.

\begin{tabular}{ll}
\hline Experimental host range & Natural host range $^{1}$ \\
\hline Amaranthaceae & Asteraceae \\
Gomphrena globosa & Santolina rosmarinifolia \\
Asteraceae & Taraxacum officinalis \\
Lactuca sativa & Boraginaceae \\
Chenopodiaceae & Anchusa azurea \\
Chenopodium amaranticolor & Cistaceae \\
Chenopodium quinoa & Cistus ladanifer \\
Cucurbitaceae & Fabaceae \\
Cucumis melo* & Anthyllis vulneraria \\
Cucumis sativus & Lupinus angustifolius \\
Fabaceae & Vicia villosa \\
Phaseolus vulgaris & Fagaceae \\
Pisum sativum & Quercus ilex \\
Solanaceae & Poaceae \\
Capsicum annuum & Bromus rubens \\
Nicotiana benthamiana & Rutaceae \\
Nicotiana clevelandii & Ruta montana \\
Nicotiana tabacum (Samsun) & \\
Solanum lycopersicum* & \\
\hline 1 Most abundant plant species present in evergreen oak forests and coexisting with mountain rue. \\
Asterisks indicate most common crops in the surrounding areas to monitored evergreen oak forests.
\end{tabular}




\subsubsection{Sequencing of the MeRV genome}

Total plant RNA of a single field-collected mountain rue (Ruta montana L.) from El Pardo, which tested positive for MeRV infection (MeRV-ParP17), was treated with TURBO DNA-free ${ }^{\text {TM }}$ Dnase (Life Technologies, Carlsbad, CA, USA). After treatment, ribosomal RNA (rRNA) was removed using the Ribo-Zero ${ }^{\mathrm{TM}}$ Plant Leaf kit (Epicenter-Illumina, Madison, WI, USA). For genome sequencing, $3 \mu \mathrm{g}$ of total RNA was used for library preparation and subjected to high-throughput Next Generation Sequencing (NGS) using the Illumina platform (HiSeq2000, 2 x $125 \mathrm{bp}$ length, at the Centre for Genomic Regulation, Barcelona, Spain), generating 20 million paired-end reads. Adapters and low-quality sequences from NGS data were removed using Seqtk (https://github.com/lh3/seqtk/). The resulting reads were assessed for sequence quality using FastQC (https://www.bioinformatics.babraham.ac.uk/projects/fastqc/). MeRV genome was assembled using a reference-guided read mapping to the phylogenetically nearest potyvirus species genome as determined in the phylogenetic characterization of potyvirus species (see section 3.2.3) utilizing Bowtie 2.0. Given that the reference genome was from a virus species different from MeRV, loose mapping criteria with the default parameters for "very-sensitive-local" alignment mode was used. This settings allowed constructing contigs using reads that do not perfectly match the reference genome (Langmead and Salzberg, 2012). Only reads with mapping quality (MAPQ) score $\geq 30$ were considered correctly aligned (Li et al., 2008; Ruffalo et al., 2011). Integrative Genome Viewer (IGV) (Thorvaldsdóttir et al., 2013) was used to visualize the mapping reads and assess genome assembly. BLAST was used to identify and remove possible chimeric contigs contained in the alignment during the assembly. Mapping quality and sequencing coverage across the reference was evaluated in Qualimap 2 (Okonechnikov et al., 2016). The consensus sequence of the MeRV genome was extracted using the Fasta Alternate Reference Maker tool in the Genome Analysis ToolKit (GATK) 3.8 (McKenna et al., 2010).

The NGS-derived MeRV genomic sequence was confirmed using the same mountain rue plant RNA preparation as template for RT-PCR, utilizing different sets of specific primers. Primer pairs were designed to produce 12 fragments in such a way that adjacent fragments overlapped by at least $100 \mathrm{nt}$, 
covering the potyvirus polyprotein (see list of primers in Table 3.5). Expand ${ }^{\mathrm{TM}}$ Reverse Transcriptase (Sigma-Aldrich, St. Louis, MO, USA) was used for retrotranscription and PCR Phusion ${ }^{A}$ High-Fidelity DNA Polymerase (New England BioLabs, Beverly, MA, USA) for PCR amplification. The 5' and 3' ends of the virus were obtained by rapid amplification of cDNA ends (RACE) using Ambion $^{\mathrm{TM}}$ FirstChoice ${ }^{\circledR}$ RLM RACE kit (Life Technologies). The fragments and ends were sequenced, and assembled with MEGA 6, revealing 100\% nucleotide identity between overlapping fragments and with the NGS-derived nucleotide sequence. The same nucleotide sequence was also obtained using RNA from purified virions as template.

Table 3.5. Species-specific primers designed to obtain the full-length genomic sequence of ParP17-MeRV.

\begin{tabular}{|c|c|c|}
\hline Primer & Sequence $\left(5^{\prime}-3^{\prime}\right)$ & Position ${ }^{1}$ \\
\hline MERV-P1 5UTR For & CGTATCATCAGAGACCCGTG & $100-119$ \\
\hline MERV-P1 5UTR Rev & CACGTACGTGTTGGAGAGCC & $314-333$ \\
\hline MERV-P1For & CATGTGAAACTGATCTTAA & $223-241$ \\
\hline MERV-P1Rev & CTATTGCAAGGGAATAGTGC & 1185-1204 \\
\hline MERV-For1 & GTAGCTAGCTGTGGTTCGG & $997-1015$ \\
\hline MERV-Rev1 & CCTTTGAGCGTTCCTCCGAC & 1968-1987 \\
\hline MERV-For2 & GCAACTTCGAAGCAGCAAAG & 1853-1872 \\
\hline MERV-Rev2 & CTGCACACTCCATGACCG & $2812-2829$ \\
\hline MERV-For3 & GCGAGTTAGATCAGTGTTG & $2753-2771$ \\
\hline MERV-Rev3 & CACCGCGGACTAGTATGTC & $3709-3727$ \\
\hline MERV-For4 & CTAAGAGGGAGTCAGACAAC & 3553-3572 \\
\hline MERV-Rev4 & CTACACGTCCAAGTCTCTG & $4522-4540$ \\
\hline MERV-For5 & GAAGGTGTCGTTGATTTCG & $4420-4438$ \\
\hline MERV-Rev5 & GCATCGTGAGCTAACAATG & $4529-5447$ \\
\hline MERV-For6 & GTGCGAGCTCAGTTGATGG & $5278-5296$ \\
\hline MERV-Rev6 & GGACACTTGAAGATACCAT & $6287-6305$ \\
\hline MERV-For7 & CATGCGCGATTACAATCC & $6126-6143$ \\
\hline MERV-Rev7 & GTCCAACGATAATCGGGTTTG & 7067-7087 \\
\hline MERV-For8 & GCTAAATTCTTCAGGCCTC & $6982-7000$ \\
\hline MERV-Rev8 & CTTGAGACCTAGTTCAGC & 7954-7971 \\
\hline MERV-For9 & GTACTGAAGAGATTGACG & $7844-7861$ \\
\hline MERV-Rev9 & CCGTCCATCATTGTCCAG & $8826-8843$ \\
\hline MERV-For10 & GTATGATGGCGTGAAAGCG & $8712-8730$ \\
\hline MERV-Rev10 & GTAGCACCTCACTAACAAG & $9288-9306$ \\
\hline
\end{tabular}

${ }^{1}$ Positions according to the NGS-derived ParP17-MeRV nucleotide genomic sequence (Acc. N. MF953305).

The open reading frame of the MeRV polyprotein was identified with ORF Finder (https://www.ncbi.nlm.nih.gov/orffinder/). PredictProtein (Rost et al., 2004) and the NCBI's conserved domain database (CDD)-Search service 
(Marchler-Bauer et al., 2007) were used to identify the putative cleavage sites and the conserved sequence domains of the polyprotein. Molecular weight of the putative viral proteins was predicted from protein sequence by using the Molecular Weight tool (https://www.bioinformatics.org/sms/prot mw.html). The genomic sequence of MeRV-ParP17 was deposited in GenBank under accession number MF953305.

\subsubsection{Phylogenetic analyses of ParP17-MeRV}

Phylogenetic relationships between ParP17-MeRV and the other members of the genus Potyvirus were analysed using the nucleotide and amino acid sequences of the viral polyprotein. To study these relationships a collection of the reference sequences of the potyvirues compiled from GenBank were obtained (Appendix 1, Table A1.5). Nucleotide and amino acid sequences of these reference potyviruses were aligned with the corresponding sequences of ParP17-MeRV using MUSCLE (Edgar, 2004). Phylogenetic trees were constructed utilizing Bayesian MCMC methods. Analysis were run as explained in section 3.3.3, but using the general time-reversible substitution model with invariant sites and a gamma distribution of among-site rate variation $\left(G T R+1+\Gamma_{4}\right)$ for nucleotide alignments, and the Whelan and Goldman (WAG) substitution model for amino acid alignment. These substitution models were the best fitted to the Potyvirus polyprotein alignment as detected by jModelTest v. 2.1.10. Maximum clade credibility (MCC) trees were summarized as in section 3.3.3.

Nucleotide identities (\%) of the complete MeRV genome with those of the two phylogenetically closest virus species were compared using SimPlot v3.5.1 (Lole et al., 1999). Plots of nucleotide identity were obtained using the MeRV genome as the query sequence, and a sliding window of $200 \mathrm{nt}$ that was moved across the alignment in steps of $20 \mathrm{nt}$. 


\subsection{Statistical analyses}

All the statistical analyses described in this section were performed using the statistical software R v.3.4.1 (R Core Team, 2017), available in http://www.rproject.org/.

\subsubsection{Comparison of floristic composition and potyvirus prevalence between seasons and ecosystems}

The differences in the plant biodiversity, as species richness, between ecosystems and in potyvirus prevalence between seasons of the same sampling cycle (summer, autumn and spring) and between sampling cycles at each season, were compared using Chi-square tests (R-library: MASS) or Fisher Exact test as needed. When necessary, the Yates correction was applied. Differences in mean values of climatic/edaphic factors (temperature, relative humidity, rainfalls, soil $\mathrm{pH}$ and soil moisture) where compared using two-sided Z-tests (R-library: BSDA).

To determine the differences in the floristic composition between ecosystems, the squared Euclidean distance was used as a measure of dissimilarity between the identity, number and relative abundance of the plant species in each location. Ward's clustering method (Ward, 1963) was utilized to obtain clustering dendograms based on these Euclidean distances (R-library: vegan).

\subsubsection{Analysis of ecological factors affecting infection risk and genetic diversity of potyviruses}

\subsubsection{Analysis at potyvirus genus-wide level}

Potyvirus prevalence was used as a measure of virus infection risk. Thus, in order to develop a predictive model that explained potyvirus infection risk, genus-wide potyvirus prevalence in evergreen oak and riparian forests was used as the response variable. Models were constructed utilizing supervised inductive Machine learning methods. Machine Learning $(\mathrm{ML})$ is an area within the Artificial Intelligence field, which is focused on improving the performance of 
a system solving a task through acquiring knowledge from the experience (Mitchell, 1997). In this thesis, datasets of classified (supervised) observations were used as the input in the ML algorithms, which retrieved mathematical patterns. These patterns allowed assigning a given class of the response trait to each observation in the dataset (inductive) and to predict the class of new data (classification). Building a ML model to predict potyvirus infection risk required to cover two steps, which are described in the following subsections.

\subsection{Model construction}

Genus-wide Potyvirus prevalence ML models were built using data of the first sampling cycle. For this, plant species at each location and visit were classified into two groups: Non-host plant species (in which potyvirus infection was never detected during the monitored period), and host species (in which potyvirus infection was detected at least once). Potyvirus prevalence in host species was categorized into four classes: uninfected host (i.e., host species for which no infected individuals were detected at a given visit/location), and infected hosts with low, medium and high potyvirus prevalence. In order to minimize biases due to imbalance in the response variable, discretization of prevalence values into the later three classes, was done such that it resulted in similar number of observations in each class. Multi-class classification models were built in which categorized prevalence was considered as the response variable. The following ecological factors were considered as predictors: location, season, plant family, plant species, plant life cycle, plant density and relative abundance, plant biomass, species richness, Shannon's index and Shannon's equitability index, soil $\mathrm{pH}$, temperature, relative humidity and rainfalls in the sampled locations (minimal, maximal and average values) and number of host plants in each location. Categorical predictors with two or more $(n)$ categories, were transformed in $n$ - 1 dichotomous variables such that one of the variables is taken as reference category and each of the variables created would enter in the model individually. Three ML algorithms adapted to support multi-class classification problems were run (R-library: RWeka): Multinomial Logistic Regression (Logistic), J48 Decision Tree (J48) and Random Forest.

Logistic regression is one of the most popular statistical methods for classification problems (Mertler and Reinhart, 2016). Unlike other statistical 
methods (e.g. ordinary linear regression), logistic regression allows for response variables that have arbitrary distributions (as it is the case of this study), rather than normal distributions, and can be transformed to conform to different assumptions about the relationships between the predictors and the response variable. The $\mathrm{J} 48$ is an algorithm that allows both numeric and nominal variables to be evaluated. This kind of model generally works better in classification problems with categorical predictors that has large number of categories, and potentially establishes complex interactions with other predictors (Quinlan, 1996), as it is the case of this thesis. The J48 algorithm creates a simple decision tree from training dataset cases, which is easily interpretable as the structure of decisions in the classifying process can be graphically represented in such tree. The decision tree tries to recursively partition the dataset into subsets by evaluating the normalized Information Gain (difference in entropy) resulting from choosing a predictor to split the data from a node in the tree. The predictor with the highest Information Gain is used on every step to define each node. The process stops when no predictor can be found that increases the Information Gain. Random Forest (Breiman, 2001) is an ensemble of techniques because combines many independent decision trees, where each tree is trained with a different subset of predictors and tested on random samples from the whole original dataset. The final output is a combination of the predictions of the individual trees. Finally, ZeroR algorithm was used as the baseline for classification accuracy. ZeroR is the simplest classification method, which depends on the response variable and ignores all predictors. This algorithm simply predicts the majority class in the data. Classification models were considered valuable if they had better performance than this baseline algorithm (better classification than expected by chance) (Witten et al., 2016).

Each of these algorithms was applied to two different categorizations of the response variable: (i) one in which non-host species plus the four categories of hosts were considered, and (ii) other in which only the four host species categories were considered. Because in both categorization the data set was imbalanced (either the number of non-host species was much larger than the number of host species, or the number of uninfected host species was larger than the number of infected species), Synthetic Minority Over-sampling 
Technique (SMOTE) (Chawla et al., 2002) was applied to increases the number of observations in minority classes of potyvirus prevalence.

\subsection{Model evaluation}

The performance (predictive power) of the ML models was evaluated by two approaches: (i) by means of five replicates of two-fold cross validation $(5 \times 2 \mathrm{CV})$ (Dietterich, 1998). In this method, the dataset of the first sampling cycle (used to build the models) was randomly split into two datasets of equal size: training and testing datasets. Then, the former dataset was used to train the model, and the later dataset was used as an unseen test set to assess the model performance. This procedure was repeated five times and the performance measures were averaged. (ii) The model constructed with data from the first sampling cycle was challenged with the data of the second sampling cycle and performance was measured.

For evaluating the performance of the ML models, measures based on a confusion matrix were used: True Positives (TP), True Negatives (TN), False Positives (FP) and False Negatives (FN) were calculated. Performance was quantified as overall model accuracy, defined as $(T P+T N) /(T P+T N+F P+F N)$, which represents the rate of correct predictions of the ML models. In imbalanced data sets, measures derived from a confusion matrix are highly dependent on the predictions for the majority class. That is, high accuracy of the model may be just due to correct classification of the majority class, but minority classes may be poorly classified. Thus, calculations of the Area Under the Receiver Operating Characteristic curve (AUROC) were also used to assess model accuracy. Importantly, AUROC values are not affected by data imbalance. This measure allowed analyzing whether, despite the use of SMOTE techniques, the ML predictive power was still affected by data imbalance. ROC curves were obtained by plotting the True Positive rate (TPR), defined as $(\mathrm{TP}) /(\mathrm{TP}+\mathrm{FN})$, against the False Positive rate (FPR), defined as $(\mathrm{FP}) /(\mathrm{FP}+\mathrm{TN})$. After plotting the ROC curve, the area under the curve was calculated. Models with no better accuracy that obtained by chance have an $A \cup R O C=0.5$, whereas highly accurate models are characterized by large areas under the ROC curve, reaching perfect accuracy when AUROC=1 (Fawcett, 2004). For minority classes of the response variable (classes of infected host 
with low, medium and high prevalence), the True Positive rate (TPR) was also calculated in order to determine the accuracy in the prediction of the less represented prevalence classes.

The relative importance of the predictors considered by the $\mathrm{ML}$ models were also analysed: in Logistic Regression models, "Stepwise AIC" method was used for selection of the most relevant factors affecting potyvirus prevalence ( $R$ library: nnet). Both stepwise-forward and stepwise-backward procedures were applied (R-library: stats): starting from null and full models, predictor variables were tested to be included or excluded at each step based on Akaike's Information Criterion (AIC). The AIC penalizes for the complexity, dropping from the model those variables do not result in a lower AIC (indicative of a better fit to the data) (Burnham and Anderson, 2004). Sequential addition and/or deletion of predictor variables continues until no further variables can be dropped without a significant loss of fit, reaching the model with the best global fit but with the minimum number of predictors. Importance of every predictor variable in the models was calculated as the increase in Nagelkerke $R^{2}$ values $\left(R^{2} N\right)$ that each variable produces when it is added to the model (Nagelkerke, 1991) (R-library: Imtest). Logistic Regression models do not assume normality, linearity or homoscedasticity. However, these models assume independence of observations, and therefore collinearity is assumed to be minimal (Hosmer et al., 2013). Predictors were tested for collinearity by calculating the variance inflation factor (VIF). For all predictors VIF values were smaller than 5, indicating the absence of collinearity. The predictive power of the resulting Logistic Regression models were evaluated using R-library: caret, and Area under the ROC curve was estimated using R-library: HandTill2001. It should be noted that $\mathrm{J} 48$ models do not provide numerical information on the relative importance of each predictor variable. However, the relative importance of the predictor variables can be derived from the position, and the frequency of appearance (number of nodes), of each predictor in the tree. Also, a single optimal tree is generated, when it is possible that other combination of predictors yield a model with similarly predictive power. At odds, Random Forest combine multiple independent decision trees to analyse the relative importance of each predictor and are therefore more robust. In Random Forest models the importance of each predictor is calculated from randomized tree 
ensembles and ranked based on Mean Decrease Impurity (MDI) measures (Louppe et al., 2013), which works similarly to Information Gain. These metrics measure the contribution of each predictor variable in decreasing the overall entropy of the ML model: the higher the increase in ML model entropy when a given predictor is eliminated from the model, the higher the relative contribution of such predictor to the accuracy of the model. These measures are normalized in a range from 0 to 1 : the higher the MDI of a given predictor variable, the more relevant such variable is.

\subsubsection{Analysis at potyvirus species-specific level}

Mixed effect multiple regression models were used to analyse the association between ecological factors, and species-specific potyvirus prevalence and population genetic diversity parameters (Burnham and Anderson, 2004). This approach, rather than ML methods, was chosen because the reduced size of the species-specific dataset resulted in ML models with low accuracy. On the other hand, species-specific datasets were large enough for the mixed approach to construct accurate models. In these analyses continuous values of the dependent variable was used and normal distribution of data was analysed by Shapiro-Wilk normality test (R-library: stats). We considered the following factors as predictors of potyvirus prevalence: host plant density and relative abundance, species richness, plant biomass, temperature, relative humidity and precipitations in the sampled locations (minimal, maximal and average values), and season. The same ecological factors, with the addition of species-specific potyvirus prevalence, were used as predictors of virus population diversity parameters. A set of models that included a global model containing all ecological factors as fixed predictors (except season that was considered as covariate), and nested models that contained all possible combinations of these predictors, were fitted for each response variable using general linear mixed models (R-library: asreml3). Models were constructed using a simultaneous autoregressive variance-covariance matrix to account for time-dependency and covariation between predictor variables. Global and nested models were ranked according to AIC, and the model with the lowest AIC score was selected as the best-ranked model. The relative importance of the predictors included in each model was calculated by residual maximum likelihood (R-library: asreml3). 
4. RESULTS 


\subsection{Characterization of evergreen oak forest and riparian forests in the Iberian Peninsula}

This thesis focuses in two wild ecosystems in the central plateau of the Iberian Peninsula: evergreen oak forests and riparian forests. These ecosystems were chosen because they are the most representative, and have the higher ecological value, among those present in this geographical area. In addition, both ecosystems are of particular agronomical interest as they are often adjacent to agroecosystems, which favors plant virus dispersal between wild and agroecosystems. Therefore, evergreen oak and riparian forests may be sources of virus epidemics in crops. Finally, a priori these ecosystems represent two extremes of a continuum among the ecosystems located in the Iberian Peninsula in terms of ecological and climatic conditions. For instance, plant species of evergreen oak forests are adapted to the extreme conditions of the Mediterranean-Continental climate, characterized by warm and dry summer seasons, and lower but mild temperatures and more abundant rainfalls in spring and autumn. The water shortage during summer season may compromise nutrient availability on plant communities in evergreen oak forests limiting plant biodiversity and biomass (Rodà, 1999). On the other hand, plant communities in riparian forest are under the influence of the nearby rivers, which results in more stable temperatures and higher relative humidity throughout the year. Under these conditions, riparian forests are generally characterized by moist and nutrient-rich soils associated with higher plant biodiversity and biomass, and a different floral composition than evergreen oak forests (Figure 4.1). These ecological and climatic factors may be determinants of pathogen infection risk and population genetic diversity (Anderson et al., 2004; Archie et al., 2009; Johnson et al., 2015). If these differences in ecological and climatic conditions between evergreen oak and riparian forests hold true in the locations monitored in this thesis, the selected ecosystems would represent a suitable system to analyse how the above-mentioned factors affect plant virus infection risk and population genetic diversity. Thus, in this chapter, differences in floral composition, ecological factors and climatic variables among locations monitored in this work are analysed. 
Evergreen oak forest
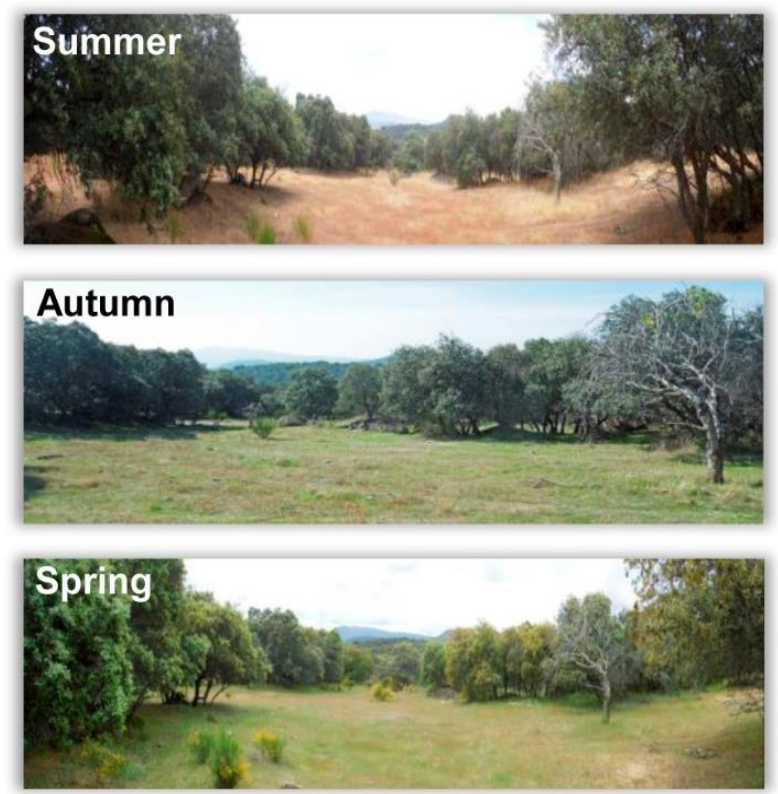

Riparian forest
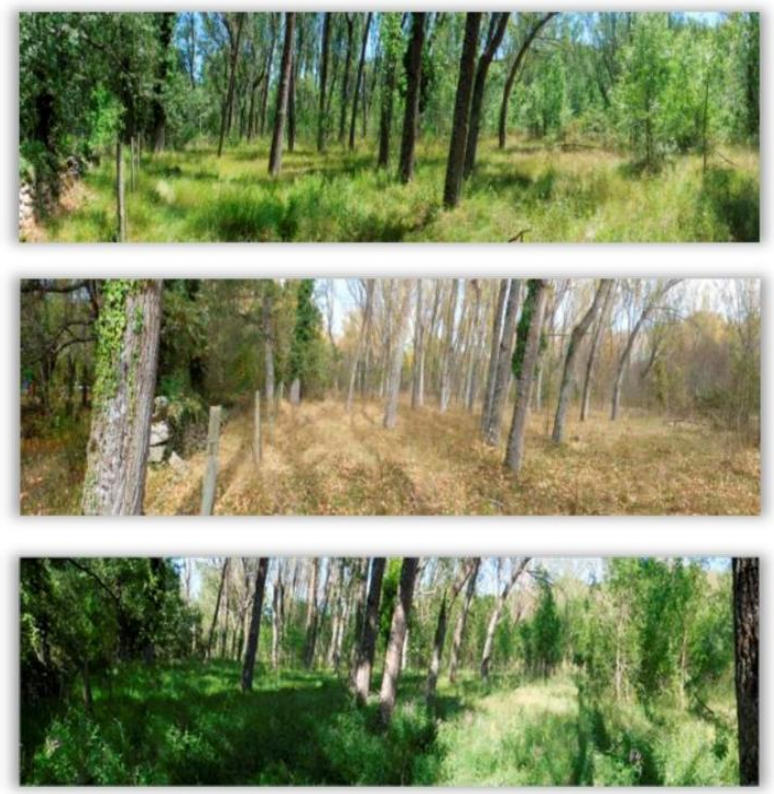

Figure 4.1. Examples of the landscape in summer, autumn and spring of evergreen oak forests (Cenicientos) and riparian forests (Rascafría) locations visited in this thesis.

\subsubsection{Analysis of plant biodiversity and floristic composition}

Plant biodiversity was quantified as species richness. Thus, for a meaningful analysis of plant biodiversity, plant sampling needs to be representative of the number of plant species (species richness) present in the analysed locations of each ecosystem. To test if this was so, an estimation of plant species richness as a function of the number of samples collected at each location and season was obtained using the non-parametric Chao 1 method (Sest) (see section 3.1.4). Estimates were compared with the observed plant species richness derived from the data collected during the field samplings ( $\mathrm{S}_{\mathrm{obs}}$ ). Using this data, accumulation curves of the number of samples to the number of plant species collected were constructed for each location and season during the first sampling cycle (2013-2014) in both ecosystems. The asymptotic values of Sest and $S_{o b s}$ curves were calculated as a measure of expected and observed plant species richness, respectively. In most locations and seasons, the 95\% confidence intervals (Cls) for asymptotic Sest contained the mean of the asyomptotic $S_{o b s}$ values, indicating that there was no significant difference between both observed and expected values in locations of evergreen oak (Figure 4.2 and Table 4.1) and riparian forests (Figure 4.3 and Table 4.2). 

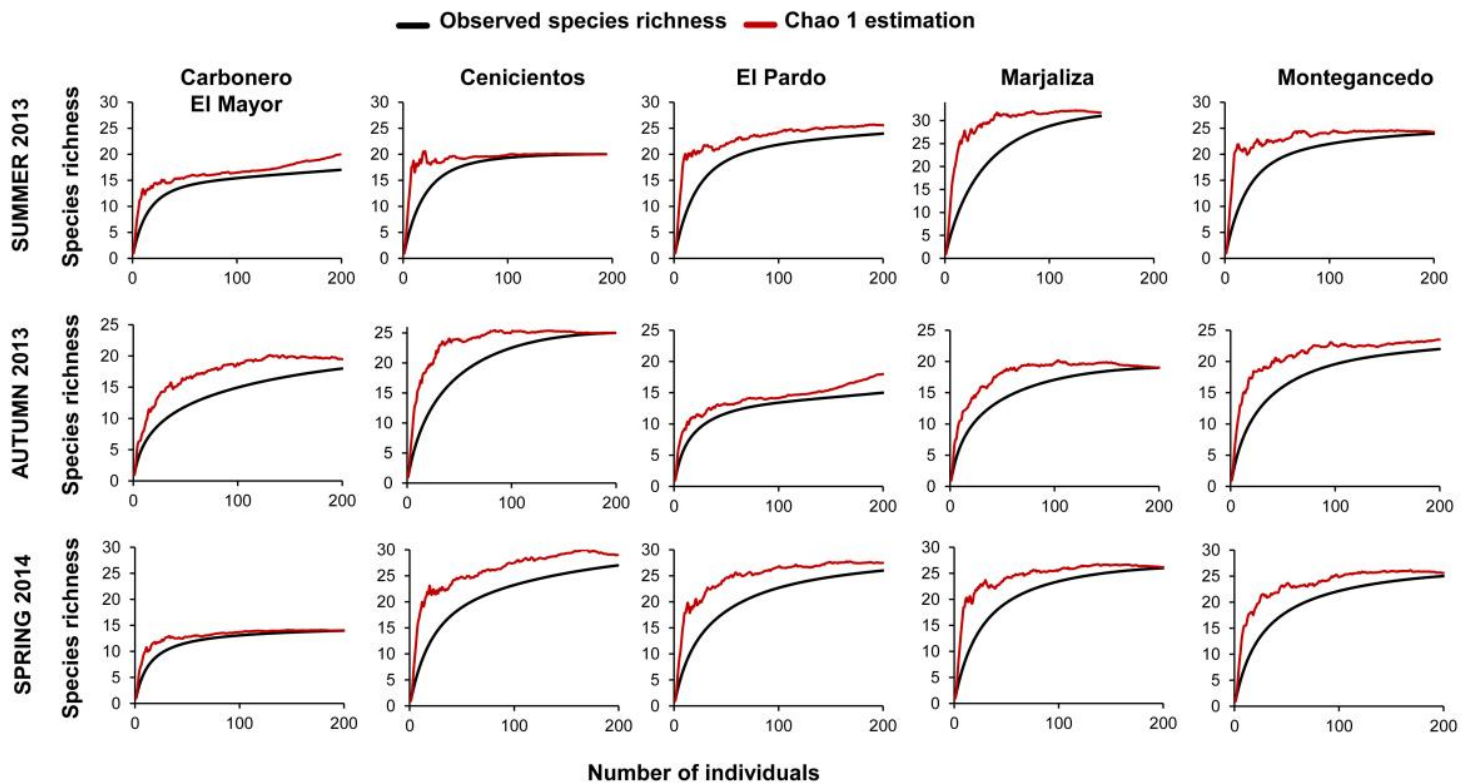

Figure 4.2. Observed ( $S_{\text {obs}}$, black) and expected ( $S_{\text {est, }}$ Chao 1 estimator, red) accumulation curves of plant species richness in the studied locations of evergreen oak forest for the monitored seasons during the 2013-2014 field samplings. The x-axis is the number of individuals sampled at each location (in this thesis, $\mathrm{N}=200$ ) and the $\mathrm{y}$-axis is the number of plant species collected according to sample size. Plotted values are means of 100 randomizations.

Table 4.1. Plant biodiversity in the studied evergreen oak forest locations during 2013-2014 field samplings. Observed and expected plant species richness, and Shannon's diversity and equitability indexes are shown.

\begin{tabular}{cccccc}
\hline Location & Season & $\mathbf{S}_{\text {obs }}(\mathbf{9 5} \% \mathbf{C l})^{\star}$ & $\mathbf{S}_{\text {est }}(95 \% \mathbf{C l})^{\star}$ & $\mathbf{H}^{\prime}$ & $\mathbf{E}_{\mathbf{H}}$ \\
\hline Carbonero \\
el Mayor & Summer 2013 & $17(13.88-20.12)$ & $19.98(17.35-42.45)$ & $2.55 \pm 0.00$ & 0.88 \\
& Autumn 2013 & $18(15.02-20.98)$ & $19.49(18.18-30.42)$ & $2.19 \pm 0.00$ & 0.76 \\
& Spring 2014 & $14(12.64-15.36)$ & $14.00(14.08-15.09)$ & $2.31 \pm 0.00$ & 0.88 \\
Cenicientos & Summer 2013 & $20(19.06-20.94)$ & $20.00(20.00-21.45)$ & $2.82 \pm 0.00$ & 0.94 \\
& Autumn 2013 & $25(24.38-25.62)$ & $25.00(26.23-26.58)$ & $2.78 \pm 0.00$ & 0.86 \\
El Pardo & Spring 2014 & $27(23.73-30.27)$ & $28.99(27.28-40.91)$ & $2.94 \pm 0.00$ & 0.89 \\
& Summer 2013 & $24(20.19-27.81)$ & $25.49(24.15-39.01)$ & $2.95 \pm 0.00$ & 0.93 \\
& Autumn 2013 & $15(11.91-18.09)$ & $17.99(15.35-40.37)$ & $2.26 \pm 0.00$ & 0.84 \\
Marjaliza & Spring 2014 & $26(22.96-29.04)$ & $27.49(28.18-38.42)$ & $2.86 \pm 0.00$ & 0.88 \\
& Summer 2013 & $31(28.95-33.05)$ & $31.74(31.08-38.19)$ & $3.14 \pm 0.00$ & 0.91 \\
& Autumn 2013 & $19(18.31-19.69)$ & $19.00(19.93-20.34)$ & $2.45 \pm 0.00$ & 0.83 \\
& Spring 2014 & $26(24.77-27.23)$ & $26.17(26.01-29.58)$ & $2.96 \pm 0.00$ & 0.91 \\
Montegancedo & Summer 2013 & $24(22.43-25.57)$ & $24.25(24.01-28.78)$ & $2.96 \pm 0.00$ & 0.93 \\
& Autumn 2013 & $22(18.22-25.78)$ & $23.49(22.15-37.01)$ & $2.62 \pm 0.00$ & 0.85 \\
& Spring 2014 & $25(22.97-27.03)$ & $25.60(25.05-32.08)$ & $2.86 \pm 0.00$ & 0.92 \\
\hline
\end{tabular}

Sobs: observed plant species richness. Sest: expected plant species richness based on the Chao 1 estimator. H': Shannon's diversity index. $E_{H}$ : Shannon's equitability index.

* values are mean and 95\% confidence intervals based in 100 randomizations from pooled locationspecific individuals $(\mathrm{N}=200)$. 


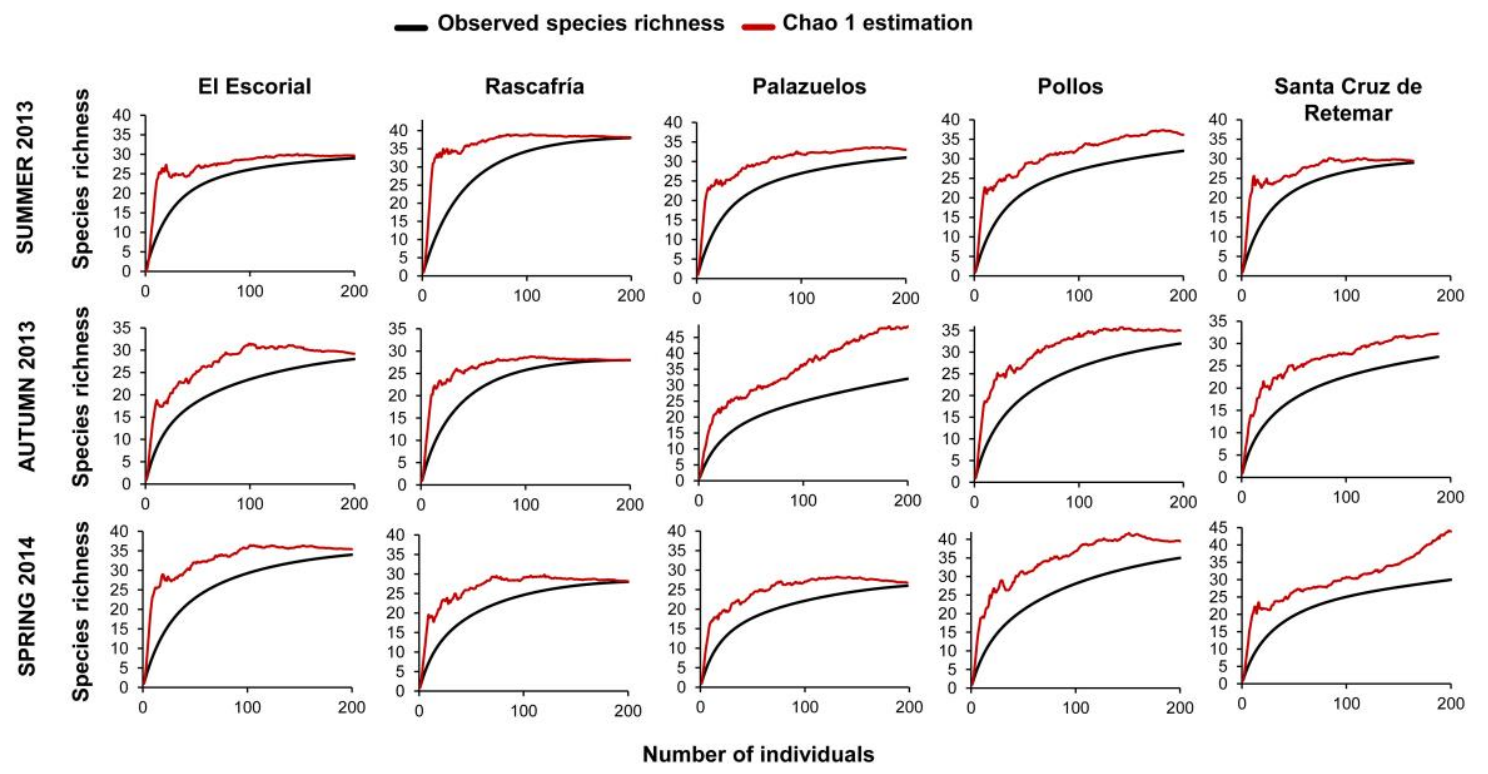

Figure 4.3. Observed (Sobs, black) and expected (Sest, Chao 1 estimator, red) accumulation curves of plant species richness in the studied locations of riparian forest for the monitored seasons during the 2013-2014 field samplings. The $x$-axis is the number of individuals sampled at each location (in this thesis, $\mathrm{N}=200$ ) and the $\mathrm{y}$-axis is the number of plant species collected according to sample size. Plotted values are means of 100 randomizations.

Table 4.2. Plant biodiversity in the studied riparian forest locations during 2013-2014 field samplings. Observed and expected plant species richness, and Shannon's diversity and equitability indexes are shown.

\begin{tabular}{cccccc}
\hline Location & Season & Sobs $_{(95 \% ~ C l)^{*}}$ & $\mathbf{S}_{\text {est }}(\mathbf{9 5 \%}$ Cl) & H' $^{*}$ & $\mathbf{E}_{\mathbf{H}}$ \\
\hline El Escorial & Summer 2013 & $29(26.97-31.03)$ & $29.60(29.05-36.08)$ & $3.15 \pm 0.00$ & 0.94 \\
& Autumn 2013 & $28(25.47-30.53)$ & $29.24(28.26-37.48)$ & $2.90 \pm 0.00$ & 0.87 \\
& Spring 2014 & $34(31.26-36.74)$ & $35.42(34.19-44.52)$ & $3.23 \pm 0.00$ & 0.92 \\
Rascafría & Summer 2013 & $38(36.88-39.12)$ & $38.14(38.01-41.23)$ & $3.47 \pm 0.00$ & 0.95 \\
& Autumn 2013 & $28(26.58-29.42)$ & $28.00(28.00-31.09)$ & $3.00 \pm 0.00$ & 0.90 \\
& Spring 2014 & $28(26.96-29.04)$ & $28.12(28.01-30.96)$ & $2.95 \pm 0.00$ & 0.89 \\
Palazuelos & Summer 2013 & $31(27.71-34.29)$ & $32.99(31.28-44.91)$ & $3.17 \pm 0.00$ & 0.92 \\
& Autumn 2013 & $32(24.75-39.25)$ & $48.42(36.23-95.65)$ & $2.92 \pm 0.00$ & 0.84 \\
& Spring 2014 & $26(23.95-28.05)$ & $26.75(26.08-33.20)$ & $2.84 \pm 0.00$ & 0.87 \\
Pollos & Summer 2013 & $32(27.57-36.43)$ & $36.18(32.79-54.24)$ & $3.15 \pm 0.00$ & 0.91 \\
& Autumn 2013 & $32(28.28-35.72)$ & $34.98(32.54-48.39)$ & $3.00 \pm 0.00$ & 0.86 \\
de Retamar & Spring 2014 & $35(30.64-39.36)$ & $39.48(35.97-55.61)$ & $3.09 \pm 0.00$ & 0.87 \\
& Summer 2013 & $29(27.33-30.67)$ & $29.43(29.03-34.55)$ & $3.14 \pm 0.00$ & 0.93 \\
& Autumn 2013 & $27(22.10-31.90)$ & $32.22(27.99-54.48)$ & $2.75 \pm 0.00$ & 0.80 \\
& Spring 2014 & $30(22.30-37.70)$ & $43.93(32.91-96.79)$ & $2.96 \pm 0.00$ & 0.87 \\
\hline
\end{tabular}

Sobs: observed plant species richness. Sest: expected plant species richness based on the Chao 1 estimator. H': Shannon's diversity index. $\mathrm{E}_{\mathrm{H}}$ : Shannon's equitability index.

* values are mean and $95 \%$ confidence intervals based in 100 randomizations from pooled locationspecific individuals $(\mathrm{N}=200)$. 
The same conclusion was obtained when observed and expected plant species richness was compared using the data of the second sampling cycle (2014-2015) for locations of evergreen oak (Appendix 2, Figure A2.1 and Table A2.1) and riparian forests (Appendix 2, Figure A2.2 and Table A2.2). Hence, this analysis indicated that the sampling effort performed in this thesis was sufficient to obtain a representative estimate of the plant species richness in the monitored ecosystems and seasons.

Next, we studied the differences in plant species richness between evergreen oak and riparian forests. Because when the two sampling cycles were analysed separately equivalent results were obtained, data on the analyses of the two cycles together are shown. Contingency tests using plant species richness as variable indicated that, within each ecosystem, the number of plant species did not vary between seasons $\left(X^{2}<3.29\right.$; $\left.P>0.069\right)$. However, in every season the number of plant species present in riparian forests was significantly higher than in evergreen oak forests $\left(X^{2}>30.24 ; P<1 \times 10^{-5}\right)$ (Tables 4.1 and 4.2 and Figure 4.4). The Shannon's diversity and equitability indexes were also higher in riparian than in evergreen oak forests, indicating that the number of plant species was higher and more evenly distributed (Tables 4.1 and 4.2).

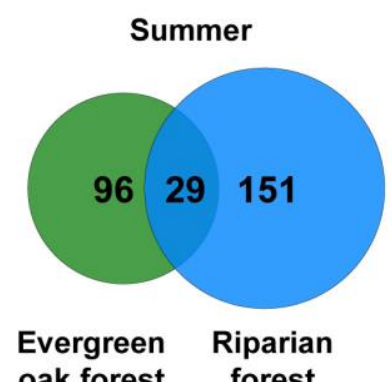

oak forest forest

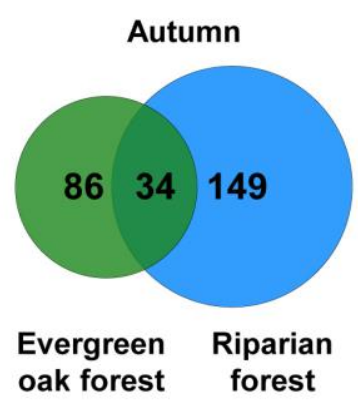

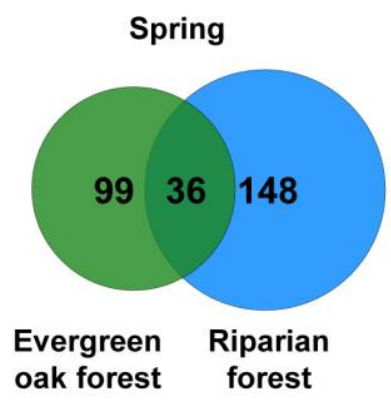

Figure 4.4. Seasonal variation in the number of plant species identified at riparian and evergreen oak forests, and number of common species to both ecosystem (2013-2015).

To characterize plant biodiversity in a given ecosystem, it is important to consider both the species richness and the species identity. A percentage of $96 \%(5731 / 6000)$ of the total plant individuals sampled in the monitored locations were identified at the species level in riparian forests and $98 \%$ 
(5877/6000) in evergreen oak forests (Appendix 2, Tables A2.3 and A2.4). Common plant species to both ecosystems represented approximately one third (30-40\%) of total plant species collected in evergreen oak forests, and one fifth (20-24\%) of those collected in riparian forests (Figure 4.4). This suggested that the identity of the species present in each ecosystem, and therefore their floristic composition, was different. To analyse this possibility, Ward's dendrograms were constructed using data on species richness, identity and relative frequency at each sampled location of evergreen oak and riparian forests (Figure 4.5). These dendrograms indicated that floristic composition at each location varied across seasons. In spite of this variation, dendrograms indicated that in every season locations always grouped according to ecosystem. Thus, evergreen oak and riparian forests had different floristic compositions.
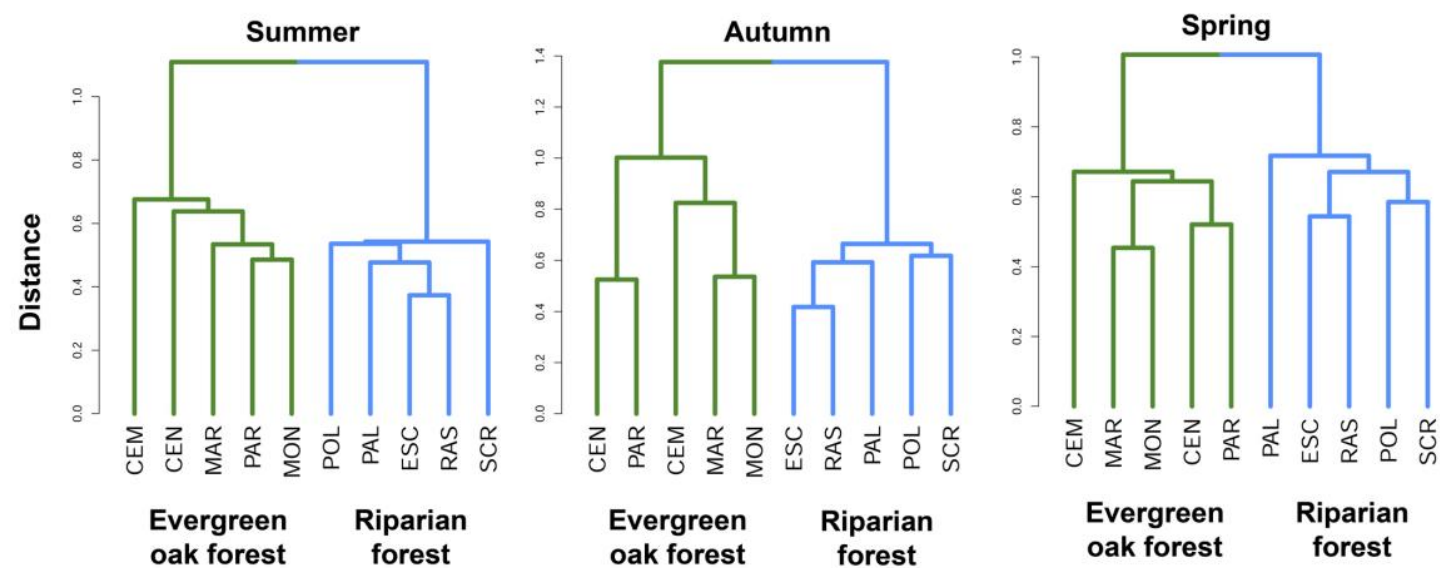

Figure 4.5. Cluster analysis of the monitored locations according to plant species identity as depicted by the Ward's dendrogram method. The vertical axis represents Euclidean distance between locations, and colours indicate the ecosystem to which each location is assigned (Evergreen oak forests: green; Riparian forests: blue). CEM: Carbonero el Mayor, CEN: Cenicientos, ESC: El Escorial, MAR: Marjaliza, MON: Montegancedo, PAL: Palazuelos, PAR: EI Pardo, POL: Pollos, RAS: Rascafría, SCR: Santa Cruz de Retamar. 
As part of the information collected on plant identity, the type of life cycle (annual or perennial) of each plant species was determined (Appendix 2, Tables A2.3 and A2.4). Using this information, the proportion of plant species in each ecosystem with different life cycles was analysed. Contingency tests indicated that, in evergreen oak forests, there were similar proportions of perennial and annual plants in summer and spring (45-49\% vs. $51-55 \%$, respectively, $\left.X^{2}<1.62 ; P>0.203\right)$, but in autumn there were more perennial than annual plants $\left(59 \%\right.$ vs. $\left.41 \%, X^{2}=5.78 ; P=0.016\right)$. In riparian forests the majority of plant species were perennials in every season $(67-74 \%$ vs. $26-33 \%$, $\left.X^{2}>21.78 ; P<1 \times 10^{-5}\right)$ (Figure 4.6).
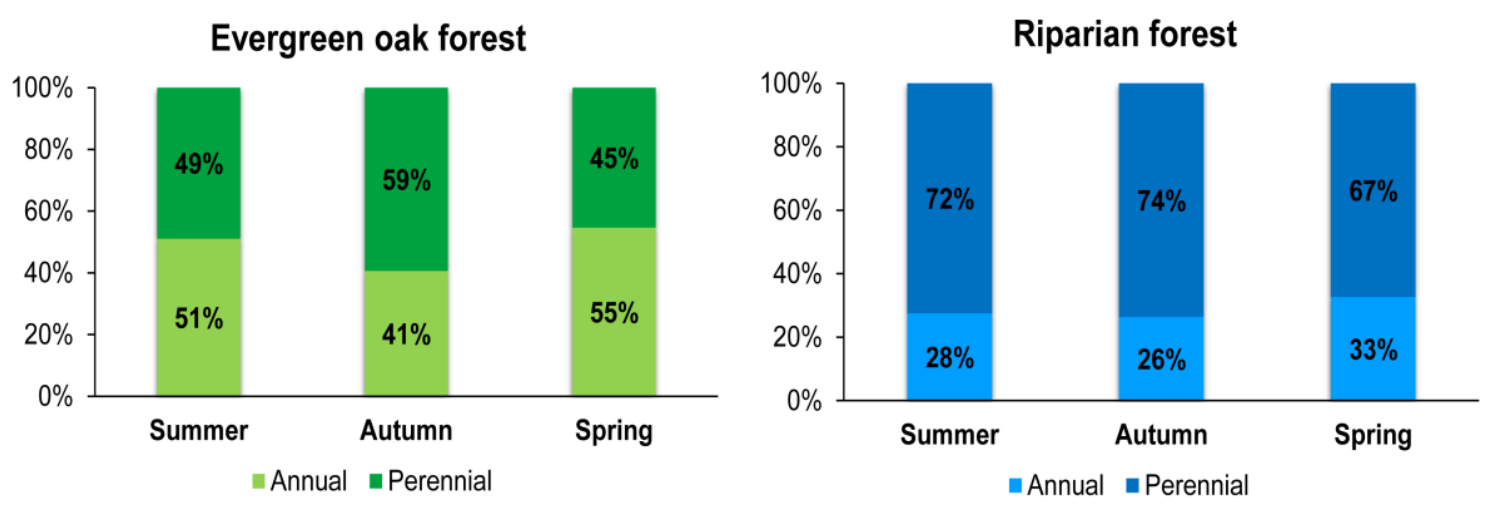

Figure 4.6. Seasonal proportion of annual and perennial plants in the studied ecosystems.

Differences in plant species richness and floristic composition may result in differences in the amount of plant biomass at each ecosystem (Blanco et al., 2005). Thus, the overall and seasonal plant biomasses at each ecosystem were calculated (Table 4.3). When Z-tests were used to compare the mean values of plant biomass between ecosystems, overall, plant biomass was larger in riparian than in evergreen oak forests $\left(V=0.119 \pm 0.10\right.$ vs. $0.056 \pm 0.05 \mathrm{~m}^{3}$; $Z=2.17, P=0.030$ ), and similar trends were observed when each season was analysed separately $(Z>1.99 ; P<0.046)$ (Table 4.3). Thus, differences in plant composition and biodiversity between ecosystems were accompanied by differences in plant biomass. 
Table 4.3. Mean values of plant biomass $\left(\mathrm{m}^{3}\right)$ in evergreen oak and riparian forests locations.

\begin{tabular}{lccc}
\hline Ecosystem/Location & \multicolumn{3}{c}{ Season } \\
\hline & Summer & Autumn & Spring \\
\cline { 2 - 4 } Evergreen oak forest & & & \\
Carbonero el Mayor & $0.054 \pm 0.06$ & $0.025 \pm 0.00$ & $0.019 \pm 0.00$ \\
Cenicientos & $0.007 \pm 0.01$ & $0.009 \pm 0.00$ & $0.081 \pm 0.00$ \\
El Pardo & $0.011 \pm 0.00$ & $0.046 \pm 0.00$ & $0.065 \pm 0.03$ \\
Marjaliza & $0.153 \pm 0.17$ & $0.047 \pm 0.04$ & $0.065 \pm 0.06$ \\
Montegancedo & $0.026 \pm 0.02$ & $0.061 \pm 0.02$ & $0.177 \pm 0.15$ \\
Average* & $\mathbf{0 . 0 5 0} \pm \mathbf{0 . 0 6}$ & $\mathbf{0 . 0 3 8} \pm \mathbf{0 . 0 2}$ & $\mathbf{0 . 0 8 1} \pm \mathbf{0 . 0 6}$ \\
& & & \\
Riparian forest & & & \\
El Escorial & $0.270 \pm 0.15$ & $0.197 \pm 0.00$ & $0.141 \pm 0.02$ \\
Palazuelos de Eresma & $0.063 \pm 0.02$ & $0.018 \pm 0.02$ & $0.075 \pm 0.00$ \\
Pollos & $0.121 \pm 0.02$ & $0.081 \pm 0.02$ & $0.137 \pm 0.01$ \\
Rascafría & $0.341 \pm 0.21$ & $0.037 \pm 0.00$ & $0.213 \pm 0.04$ \\
Santa Cruz de Retamar & $0.010 \pm 0.01$ & $0.038 \pm 0.04$ & $0.043 \pm 0.04$ \\
Average* $^{*}$ & $\mathbf{0 . 1 6 1 \pm \mathbf { 0 . 1 4 }}$ & $\mathbf{0 . 0 7 4} \pm \mathbf{0 . 0 7}$ & $\mathbf{0 . 1 2 1} \pm \mathbf{0 . 0 7}$ \\
\hline
\end{tabular}

${ }^{*}$ Biomass values based in grouping data from all locations of the ecosystem $\left(\mathrm{m}^{3}\right)$.

Values are mean \pm standard deviations based in data registered between 2013 and 2015 .

4.1.2. Analysis of variation in abiotic factors between riparian and evergreen oak forests

\subsubsection{Climatic factors}

The following climatic factors were recorded in the monitored locations: monthly maximum, minimum and mean temperature $\left({ }^{\circ} \mathrm{C}\right)$, maximum, minimum and mean relative humidity (\%), and rainfalls $(\mathrm{mm})$ in the summer, autumn and spring months. In evergreen oak forests, analysis of variation in these climatic factors among seasons showed that summer was the driest season, registering the least rainfalls $\left(Z>5.17 ; P<1 \times 10^{-5}\right)$ and the lowest values of relative humidity ( $Z>2.46 ; P<0.014$ ) (Table 4.4 and Figure 4.7). Autumn was the wettest season with the highest values of rainfalls $(Z>2.55 ; P<0.011)$ and relative humidity ( $Z>3.08 ; P<0.002$ ). Summer had the highest maximum, mean and minimum temperatures ( $\left.Z>4.69 ; P<1 \times 10^{-5}\right)$, with no significant differences between spring 
and autumn $(Z<0.86 ; P>0.220)$ (Table 4.4 and Figure 4.7). In riparian forests, summer was also the driest season, with the lowest values of rainfalls $(Z>4.29$; $\left.P<1 \times 10^{-5}\right)$ and relative humidity $(Z>2.17 ; \quad P<0.030)$ and the highest temperatures $(Z>2.05 ; P<0.041)$. Autumn had higher temperatures and relative humidity than spring $(Z>2.05 ; P<0.041)$. No differences were observed in the rainfalls between spring and autumn $(Z=1.00, P=0.315)$ (Table 4.4 and Figure 4.7).

Table 4.4. Averaged seasonal values for the climatic factors recorded in the monitored locations.

\begin{tabular}{|c|c|c|c|c|c|c|}
\hline \multirow[b]{2}{*}{$\begin{array}{l}\text { Climatic } \\
\text { factor }\end{array}$} & \multicolumn{2}{|c|}{ Summer } & \multicolumn{2}{|c|}{ Autumn } & \multicolumn{2}{|c|}{ Spring } \\
\hline & $\begin{array}{l}\text { Evergreen } \\
\text { oak forest }\end{array}$ & $\begin{array}{l}\text { Riparian } \\
\text { forest }\end{array}$ & $\begin{array}{l}\text { Evergreen } \\
\text { oak forest }\end{array}$ & $\begin{array}{l}\text { Riparian } \\
\text { forest }\end{array}$ & $\begin{array}{l}\text { Evergreen } \\
\text { oak forest }\end{array}$ & $\begin{array}{l}\text { Riparian } \\
\text { forest }\end{array}$ \\
\hline $\mathrm{T}_{\min }$ & $9 \pm 2.48$ & $7 \pm 3.25$ & $3 \pm 2.75$ & $3 \pm 2.91$ & $2 \pm 1.52$ & $1 \pm 2.48$ \\
\hline$T_{\text {mean }}$ & $21 \pm 2.14$ & $20 \pm 2.77$ & $15 \pm 1.59$ & $15 \pm 1.44$ & $13 \pm 1.26$ & $13 \pm 2.07$ \\
\hline $\mathbf{T}_{\max }$ & $34 \pm 1.77$ & $33 \pm 2.22$ & $27 \pm 1.85$ & $28 \pm 1.40$ & $26 \pm 1.78$ & $26 \pm 2.15$ \\
\hline $\mathbf{R} \mathbf{H}_{\min }$ & $14 \pm 2.32$ & $14 \pm 1.41$ & $25 \pm 3.29$ & $23 \pm 5.35$ & $17 \pm 3.36$ & $17 \pm 1.72$ \\
\hline $\mathbf{R H}_{\text {mean }}$ & $40 \pm 4.38$ & $43 \pm 5.37$ & $63 \pm 6.55$ & $64 \pm 3.21$ & $54 \pm 6.08$ & $57 \pm 4.19$ \\
\hline $\mathbf{R} \mathbf{H}_{\max }$ & $91 \pm 4.61$ & $95 \pm 2.44$ & $97 \pm 3.92$ & $99 \pm 0.76$ & $96 \pm 4.30$ & $98 \pm 1.07$ \\
\hline Rainfalls & $85 \pm 36.63$ & $176 \pm 90.98$ & $493 \pm 246.80$ & $521 \pm 237.10$ & $283 \pm 81.15$ & $387 \pm 348.03$ \\
\hline
\end{tabular}

Seasonal differences between evergreen oak and riparian forests in climatic factors were also compared. No significant differences were found in the minimum, mean and maximum seasonal temperatures between both ecosystems $(Z<1.23 ; P>0.113)$. Similarly, maximum, minimum and mean relative humidity and rainfalls did not differ between ecosystems $(Z<2.06$; $P>0.039$ and $Z<0.92 ; P>0.358$ respectively). The only exceptions were that summer rainfall was larger in riparian forest $(Z=2.06 ; P=0.039)$, accompanied also by larger maximum relative humidity in this season $(Z=2.06 ; P=0.040)$ (Table 4.4 and Figure 4.7). Thus, at odds with the ecological factors analysed in section 4.1.1, climatic factors did not generally varied between evergreen oak and riparian forests. 
Summer

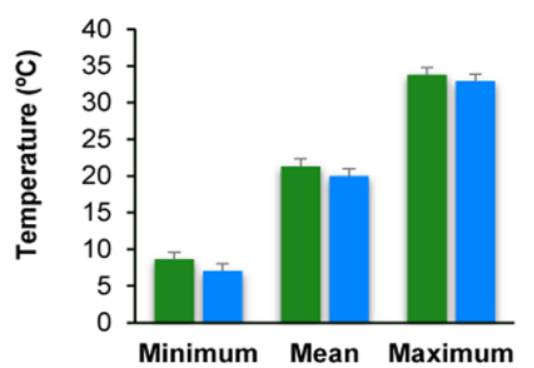

Autumn

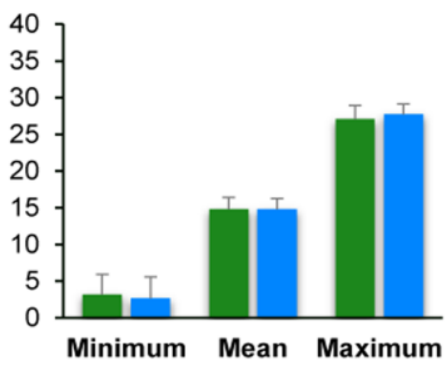

Spring

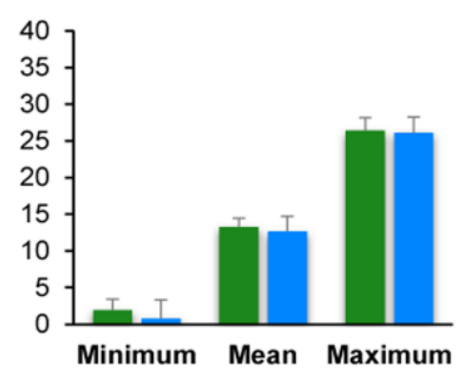

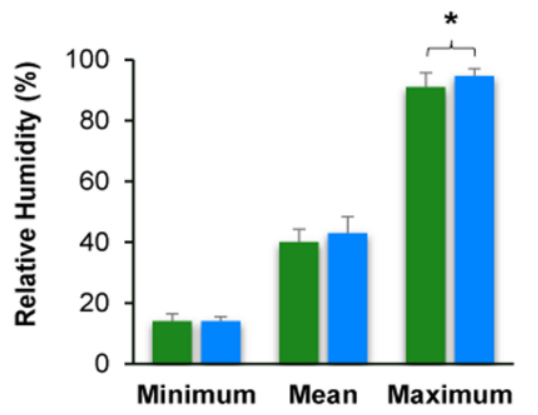
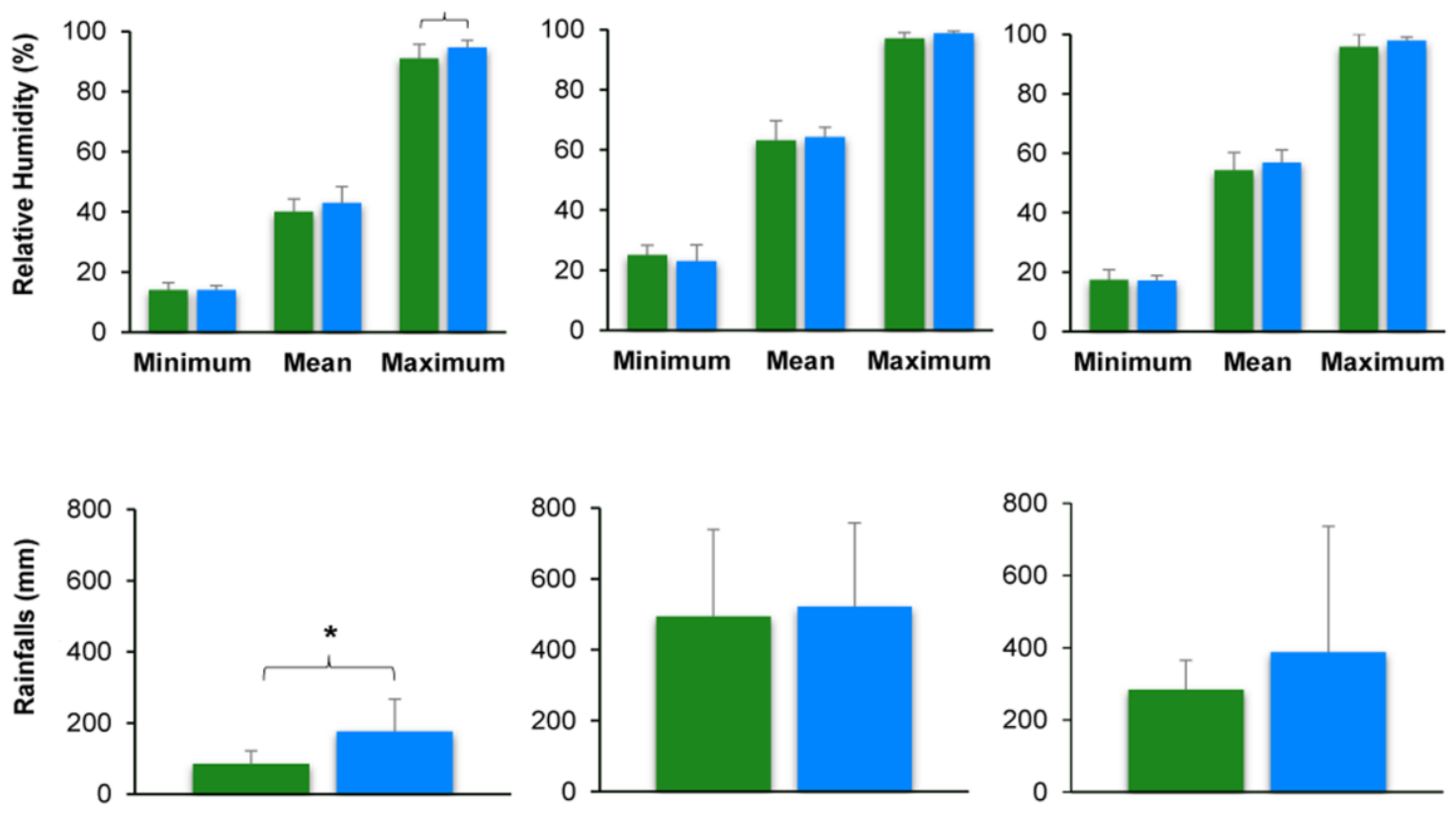

Evergreen oak forest

- Riparian forest

Figure 4.7. Seasonal differences in climatic variables between ecosystems. Asterisks indicate statistically significant differences.

\subsubsection{Edaphic factors}

Besides climatic factors, variation in the edaphic factors soil $\mathrm{pH}$ and moisture between ecosystems were also analysed. For soil $\mathrm{pH}$, no differences between seasons were detected, as soil $\mathrm{pH}$ remained constant throughout the monitored period $(Z<0.49 ; \quad P>0.628)$ (Table 4.5). Z-tests indicated no significant differences in the soil $\mathrm{pH}$ values between both ecosystems $(Z=0.98 ; P=0.327)$ and between locations of the same ecosystem $(Z<0.96 ; P>0.359)$. 
Table 4.5. Averaged values of soil $\mathrm{pH}$ in the monitored locations.

\begin{tabular}{cccc}
\hline $\begin{array}{c}\text { Evergreen oak forest } \\
\text { locations }\end{array}$ & $\mathbf{p H}^{\mathbf{1}}$ & $\begin{array}{c}\text { Riparian forest } \\
\text { locations }\end{array}$ & $\mathbf{p H}^{\mathbf{1}}$ \\
\hline Carbonero el Mayor & $5.5 \pm 0.00$ & El Escorial & $5.5 \pm 0.00$ \\
Cenicientos & $4.5 \pm 0.00$ & Palazuelos & $5.5 \pm 0.00$ \\
El Pardo & $6.0 \pm 0.00$ & Pollos & $5.0 \pm 0.00$ \\
Marjaliza & $6.0 \pm 0.00$ & Rascafría & $5.5 \pm 0.00$ \\
Montegancedo & $6.0 \pm 0.00$ & Santa Cruz de Retamar & $5.5 \pm 0.00$ \\
\hline
\end{tabular}

${ }^{1}$ Values are mean \pm standard deviation based in data registered between 2013 and 2015.

Volumetric water content (VWC) of the soil was used as measure of soil moisture in each location and season. In this case, Z-tests indicated that average soil moisture was significantly higher in riparian forests than in evergreen oak forests in every season $\left(Z>4.91 ; P<1 \times 10^{-5}\right)$. Significant differences were also registered between seasons in each ecosystem: highest soil moisture was registered in autumn, whereas driest soils were found in summer in both ecosystems $(Z>3.23 ; P<0.001)$.

Table 4.6. Averaged values of soil moisture registered in the monitored locations.

\begin{tabular}{lccc}
\hline Ecosystem/Location & \multicolumn{3}{c}{ Season } \\
\hline & Summer & Autumn & Spring \\
\cline { 2 - 4 } Evergreen oak forest & & & \\
Carbonero el Mayor & $2.60 \pm 0.47$ & $6.58 \pm 0.77$ & $3.59 \pm 0.46$ \\
Cenicientos & $1.28 \pm 0.35$ & $8.79 \pm 1.51$ & $1.65 \pm 0.92$ \\
El Pardo & - & $9.13 \pm 1.71$ & $3.58 \pm 0.71$ \\
Marjaliza & - & $3.36 \pm 1.14$ & $2.78 \pm 0.77$ \\
Montegancedo & $1.66 \pm 0.39$ & $10.80 \pm 4.30$ & - \\
Average $^{\star}$ & $\mathbf{1 . 8 1} \pm \mathbf{0 . 6 8}$ & $\mathbf{7 . 7 3} \pm \mathbf{3 . 4 4}$ & $\mathbf{2 . 9 0} \pm \mathbf{0 . 7 9}$ \\
& & & \\
Riparian forest & & & \\
El Escorial & $5.04 \pm 1.77$ & $19.74 \pm 3.21$ & $12.11 \pm 9.17$ \\
Palazuelos & $6.95 \pm 0.47$ & $22.94 \pm 3.33$ & $9.99 \pm 0.62$ \\
Pollos & - & - & $13.13 \pm 12.09$ \\
Rascafría & $3.75 \pm 1.25$ & $20.48 \pm 3.08$ & $8.01 \pm 1.13$ \\
Santa Cruz de Retamar & $2.76 \pm 0.31$ & $9.80 \pm 2.77$ & $1.64 \pm 0.41$ \\
Average $^{\star}$ & $\mathbf{4 . 8 7} \pm \mathbf{2 . 3 4}$ & $\mathbf{1 8 . 2 4} \pm \mathbf{5 . 8 9}$ & $\mathbf{8 . 9 8} \pm \mathbf{7 . 6 6}$ \\
\hline Voluming & & & \\
\end{tabular}

*Volumetric water content values based in data of all locations in the ecosystem (\%). Missing values (-). Values are mean \pm standard deviations based in data registered between 2013 and 2015 . 


\subsection{Analysis of genus-wide potyvirus prevalence in wild ecosystems of the Iberian Peninsula}

Current understanding of plant virus biodiversity and host interactions is largely restricted to viruses isolated from crops. However, recent studies have shown that plant viruses are widespread and highly diverse in wild ecosystems (Roossinck, 2012; Stobbe and Roossinck, 2014). Few studies however have investigated the genetic diversity of plant virus populations in wild ecosystems and their prevalence in native wild hosts (Malpica et al., 2006; Pagán et al., 2010; Rodríguez-Nevado et al., 2017). In this thesis, the presence of species of the genus Potyvirus was investigated in evergreen oak and riparian forests of the lberian Peninsula. This is the largest genus of plant viruses and contains species that are major crop pathogens (e.g. Walsh and Jenner, 2002; Quenouille et al., 2013), together with species that have been reported to infect wild hosts (Malpica et al., 2006; Rodríguez-Nevado et al., 2017). This suggests that potyviruses may be important ecological agents in wild ecosystems (perhaps including evergreen oak and riparian forests), and with potential to cause epidemics in adjacent crops.

In this chapter, the association between the ecological factors that characterize evergreen oak and riparian forests (analysed in section 4.1) and potyvirus prevalence is analyzed, with the ultimate goal of building a predictive model of virus prevalence in both ecosystems. Multivariate models aiming at predicting virus prevalence in cultivated hosts have been shown to represent a powerful tool to predict viral diseases in crops (Thackray et al., 2004). However, examples of similar models in wild ecosystems are scarce, especially for plant viruses (Pagán et al., 2012). In addition, these studies generally used linear or multiple linear regressions and focused on the effect of few ecological variables in the prevalence of a single viral species infecting one or few focal hosts (Pagán et al., 2016). However, relationships between ecological factors and virus prevalence are not necessarily linear, and considering only part of the virus host range may miss relevant interactions for virus prevalence. To broaden the scope of previous works, here the role of a larger variety of factors 
as predictors of potyvirus prevalence in evergreen oak and riparian ecosystems is analysed (see section 3.5.2.1.1), and the complete host range of the identified potyviruses is considered. Also, Machine Learning-based methods $(\mathrm{ML})$ are used to build predictive models of genus-wide potyvirus prevalence. ML methods have the advantage that they model complex nonlinear relationships without having to satisfy the assumptions required by traditional statistical methods (Olden et al., 2008; Thessen, 2016). For the purpose of this thesis, these methods allow studying complex relationships, as they consider both the effect of host-virus interactions and the influence of the environment in virus epidemiology.

\subsubsection{Potyvirus prevalence and host abundance in evergreen oak and riparian forests}

To analyze potyvirus prevalence, $83 \%(9,934 / 12,000)$ of the samples collected in this thesis were used. For the rest of the samples, appropriate RNA extracts could not be obtained because the nature or the preservation state of the sample did not allow it (i.e., samples from plant species that were rich in phenolic compounds or polysaccharides, or samples that were poorly preserved). Infection by potyvirus species was detected in spring, summer and autumn during the monitored period, and prevalence was calculated as the percentage of infected individuals relative to the total number of analysed plants.

Average potyvirus prevalence ranged between $1.06 \%$ and $3.43 \%$ in evergreen oak forests, and between $2.43 \%$ and $7.56 \%$ in riparian forests, depending on the season and the sampling year (Figure 4.8A and B). In the first sampling year (2013-2014), the comparison of potyvirus prevalence in evergreen oak forests between seasons indicated that prevalence was significantly higher in autumn than in summer $\left(3.35 \%\right.$ vs. $1.06 \%, X^{2}=7.04 ; P=$ 0.008 ), with intermediate prevalence values in spring that did not differ from those in the other two seasons $\left(1.87 \%, X^{2}<2.52 ; P>0.112\right)$. In riparian forests, the highest potyvirus prevalence was observed in summer $\left(7.56 \%, X^{2}>8.14\right.$; $P<0.004$ ), whereas no significant differences were observed between autumn and spring (2.62\% and $4.17 \%$, respectively, $\left.X^{2}=2.39 ; P=0.122\right)$ (Figure 4.8A). 
In the second sampling year (2014-2015), the highest potyvirus prevalence in evergreen oak forests was detected in summer $\left(3.43 \%, X^{2}>4.37 ; P<0.036\right)$, whereas no significant differences were observed between autumn and spring $\left(1.72 \%\right.$ vs. $\left.1.27 \%, X^{2}=0.35 ; P=0.551\right)$. No significant differences between seasons were observed in riparian forests during this sampling year $\left(X^{2}<0.79\right.$; $P>0.373$ ) (Figure 4.8B).
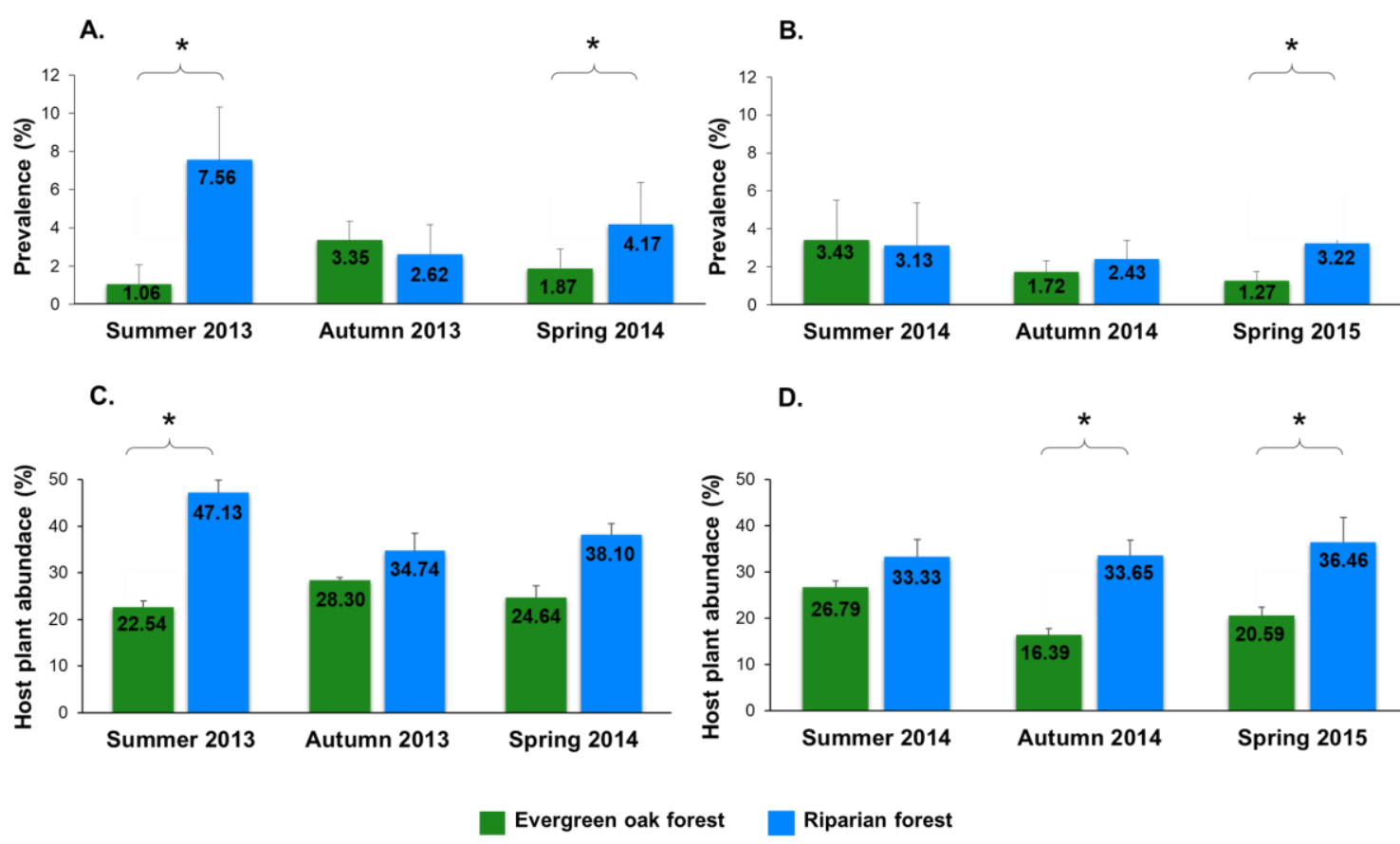

D.

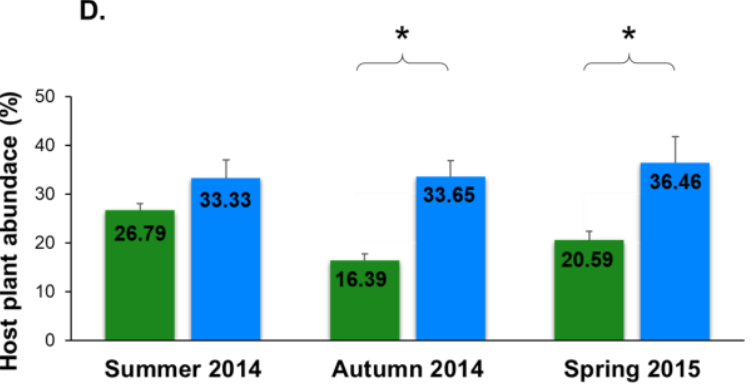

Figure 4.8. Seasonal potyvirus prevalence and host plant abundance in evergreen oak forests (green) and riparian forests (blue) during the 2013-2014 sampling cycle (A and C), and during the 2014-2015 sampling cycle (B and D). Asterisks indicate significantly different potyvirus prevalence between ecosystems.

Differences in potyvirus prevalence at each season between sampling years were also analyzed. Fluctuations in potyvirus prevalence were observed only in summer: $7.56 \%$ vs. $3.13 \%\left(X^{2}=16.36 ; P<1 \times 10^{-5}\right)$ in riparian forests, and $1.06 \%$ vs. $3.43 \%\left(X^{2}=7.97 ; P=0.005\right)$ in evergreen oak forests. There were no significant changes in virus prevalence between years in the other seasons $\left(X^{2}<3.58 ; P>0.058\right)$ (Figure 4.8A and B). The comparison between ecosystems indicated that in the first sampling cycle, potyvirus prevalence was significantly higher in riparian forests than in evergreen oak forests in spring $(4.17 \% \mathrm{vs}$. $1.87 \%$, respectively, $\left.X^{2}=6.33 ; P=0.012\right)$ and summer $(7.56 \%$ vs. $1.06 \%$, $\left.X^{2}=33.36 ; P<1 \times 10^{-5}\right)$, but not in autumn $\left(2.62 \%\right.$ vs. $\left.3.35 \%, X^{2}=0.413 ; P=0.520\right)$ (Figure 4.8A). In the second sampling cycle, the comparison of seasonal 
changes in potyvirus prevalence between ecosystems showed largely similar results than in the first sampling year: In spring, virus prevalence was significantly higher in riparian than in evergreen oak forests $(3.22 \%$ vs. $1.27 \%$, $X^{2}=7.26 ; P=0.007$ ), and no significant differences were observed in autumn (1.72\% vs. $\left.2.43 \% ; X^{2}=0.80 ; P=0.371\right)$. The exception was the potyvirus prevalence in summer that, at odds with the first sampling cycle, did not differ between ecosystems (3.43\% vs. 3.13\%, $X^{2}=0.050 ; P=0.822$ ) (Figure 4.8B).

The analysis of potyvirus prevalence allowed identifying virus host species in evergreen oak and riparian forests. Thus, changes in host plant abundance - as the number of host species relative to total number of plant species - between seasons, sampling cycle and ecosystems were analyzed. Host plant abundance in evergreen oak forests ranged between $16.39 \%$ (10/61) and $28.30 \%(15 / 53)$, and in riparian forests between $33.33 \%(38 / 114)$ and $47.17 \%$ (41/87), depending on the season and the sampling cycle (Figure 4.8C and D). In the first sampling cycle, the comparison between seasons showed that host abundance was similar in summer, autumn and spring both in evergreen oak forests $\left(22.54 \%, 28.30 \%\right.$ and $24.64 \%$, respectively, $x^{2}<0.27$; $P>0.600)$ and in riparian forests $\left(47.13 \%, 34.74 \%\right.$ and $38.10 \%, X^{2}<2.39$; $P>0.121$ ) (Figure 4.8C). Similar results were obtained in the second sampling cycle: $26.79 \%, 16.39 \%$ and $20.59 \%\left(X^{2}<1.31 ; P>0.252\right)$ in evergreen oak forests, and $33.33 \%, 33.65 \%$ and $36.46 \%\left(X^{2}<0.11 P>0.743\right)$ in riparian forests (Figure 4.8D). Also, in each ecosystem host plant abundance was similar in both sampling cycles $\left(X^{2}<3.38, P>0.066\right)$ (Figure $4.8 \mathrm{C}$ and $\mathrm{D}$ ).

The comparison of the abundance of host plants between ecosystems indicated that, in the first sampling cycle, host abundance in summer was higher in riparian than in evergreen oak forests $\left(47.13 \%\right.$ vs. $22.54 \%, X^{2}=9.21$, $P=0.002$ ) (Figure $4.8 \mathrm{C}$ ), co-occurring with the higher potyvirus prevalence in the former ecosystem (Figure 4.8A). In the other two seasons, there were no significant differences in host abundance between ecosystems $\left(x^{2}<2.84\right.$; $P>0.092)$. In the second sampling cycle, host abundance was higher in riparian than in evergreen oak forests both in autumn (33.65\% vs. $16.39 \%, X^{2}=4.94$, $P=0.026)$ and in spring (36.46\% vs. $\left.20.59 \%, X^{2}=4.06, P=0.044\right)$ (Figure 4.8D). Higher host abundance in riparian forest during spring co-occurred with higher 
potyvirus prevalence in this ecosystem (Figure 4.8B). No differences in host abundance between ecosystems were observed in summer $\left(X^{2}=0.48 ; P=0.49\right)$.

Finally, differences in the proportion of host plant species at each ecosystem according to their life cycle (annual or perennial) were also analysed. In evergreen oak forests, there was similar proportion of annual and perennial hosts at every season and sampling cycle $\left(X^{2}<0.20 ; P>0.654\right)$. At odds, in riparian forests the majority of host species were perennials $\left(X^{2}>31.25\right.$; $P<1 \times 10^{-5}$ ) (Figure 4.9).

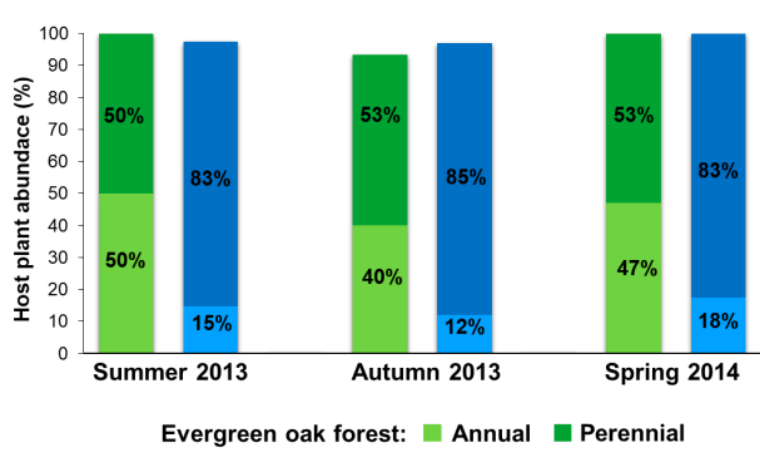

B.

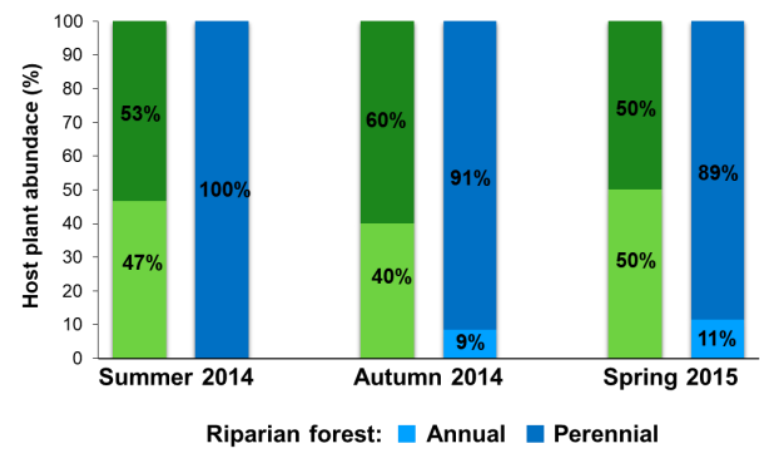

Figure 4.9. Seasonal proportion of annual and perennial host plants in evergreen oak forests (green) and riparian forests (blue) during the 2013-2014 (A) and the 2014-2015 (B) sampling cycles.

\subsubsection{Ecological factors affecting Potyvirus genus-wide prevalence}

ML methods were applied to build a model that best explained genus-wide potyvirus prevalence in evergreen oak and riparian forests. The following ecological factors were used as predictors: location of the sampled area, season, plant family, plant species, plant life cycle, plant density and relative abundance, plant biomass, plant species richness (S), Shannon's index ( $\left.H^{\prime}\right)$ and Shannon's equitability index $\left(\mathrm{E}_{\mathrm{H}}\right)$, soil $\mathrm{pH}$, minimum, maximum and average values of temperature and relative humidity and rainfalls and number of host plants in each location.

Non-normal distribution of the potyvirus prevalence data due to high proportion of non-host and non-infected plants prevented building accurate regression models using potyvirus prevalence as a continuous variable $(r \leq 0.35$; $P \geq 0.350$ ). Therefore, $M L$ models were built considering genus-wide potyvirus 
prevalence as a categorical variable. To do so, plant species at each location and visit were classified into two major groups: Non-host plant species (in which potyvirus infection was never detected during the monitored period), and host species (in which potyvirus infection was detected in at least one individual at one of the visits). Analysis of the frequency distribution of potyvirus prevalence in plant species of both riparian and evergreen oak forests, showed a high percentage of non-host species (56\% and 69\%, respectively) (Figure 4.10). At each location and visit, host species were further divided into two groups: (i) Uninfected hosts (i.e., samples from plant species that are hosts but with no infected individuals at a given visit/location), which constituted the second most abundant class in the distribution (33\% and 25\%) (Figure 4.10). (ii) Infected hosts (i.e., host species with at least one infected individual at a given visit/location). Finally, infected hosts were distributed into three prevalence classes: low, medium and high potyvirus prevalence (Figure 4.10). These three classes were created by calculating the frequency distribution of potyvirus prevalence across infected hosts and estimating the terciles of the distribution such three classes with similar number of observations in each prevalence class were created. Importantly, this categorical classification allowed minimizing biases due to imbalance in the number of instances at each category of prevalence. Potyvirus prevalence thresholds for each of these classes are shown in Table 4.7.
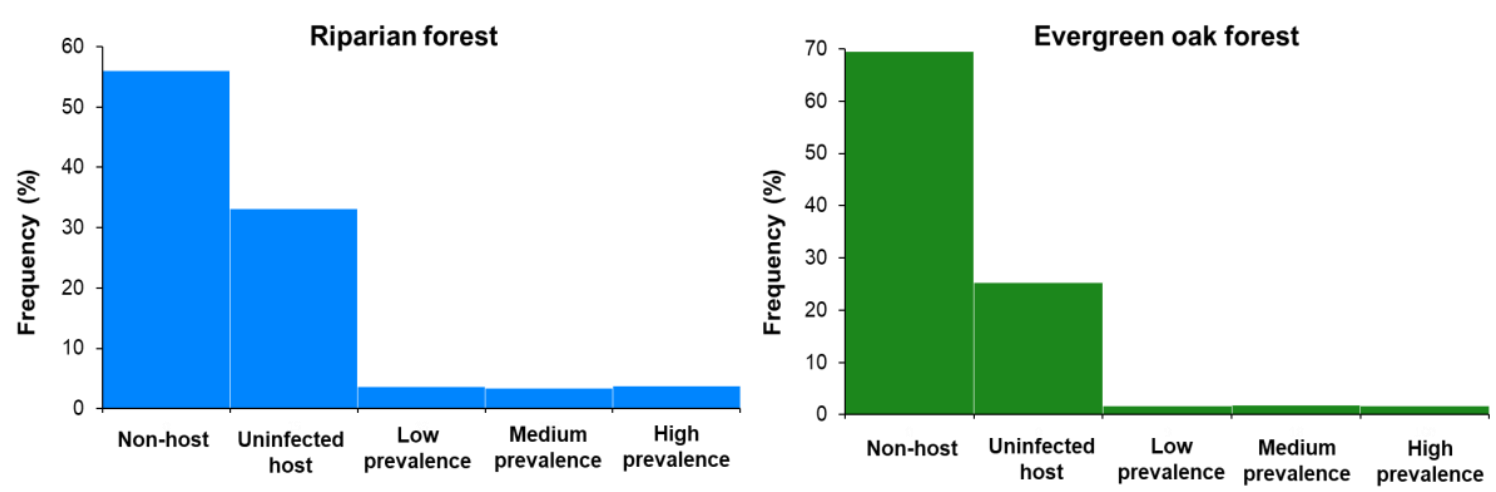

Figure 4.10. Histograms of potyvirus prevalence frequency distribution in riparian forest (blue) and evergreen oak forest (green). 
Table 4.7. Thresholds used to categorize infected hosts in low, medium and high prevalence classes in the studied ecosystems.

\begin{tabular}{cc}
\hline Riparian forest & Evergreen oak forest \\
\hline $0 \%<$ low prevalence $<15.00 \%$ & $0 \%<$ low prevalence $<9.09 \%$ \\
$15.00 \% \leq$ medium prevalence $<33.33 \%$ & $9.09 \% \leq$ medium prevalence $<18.18 \%$ \\
$31.33 \% \leq$ high prevalence $\leq 100 \%$ & $18.18 \% \leq$ high prevalence $\leq 100 \%$ \\
\hline
\end{tabular}

Unfortunately, similar balancing between prevalence classes and nonhost/uninfected classes could not be attained. Non-hosts constituted the majority class representing between $54 \%-72 \%$ of the observations, followed by uninfected hosts that represented between 22-34\%. Minority classes corresponded to infected hosts with low, medium and high potyvirus prevalence (Table 4.8). Thus, for the construction of the models, these imbalances were minimized by applying Synthetic Minority Over-sampling Technique (SMOTE) (Nitesh et al., 2002), which increases the number of observations in the minority classes of potyvirus prevalence in the training dataset (section 3.5.2.1.1).

$\mathrm{ML}$ models were constructed and trained using datasets containing information collected during the first sampling cycle (training set). The performance (predictive power) of the models was evaluated by means of five replicates of two-fold cross validation $(5 \times 2 \mathrm{CV})$ on training sets. Also, the model constructed with data from the first sampling cycle was challenged with the data of the second sampling cycle (validation set), and performance was measured (section 3.5.2.1.2). Table 4.8 shows the distribution of plant species in evergreen oak and riparian forests across the five categories of potyvirus prevalence in the training and validation data sets.

Table 4.8. Number and relative percentage (in parenthesis) of plant species in each class of potyvirus prevalence in the training and validation datasets used to build ML models.

\begin{tabular}{|c|c|c|c|c|c|}
\hline \multicolumn{3}{|c|}{ Riparian forest datasets } & \multicolumn{3}{|c|}{ Evergreen oak forest datasets } \\
\hline Prevalence class & $\begin{array}{c}\text { Training } \\
\text { set }^{1}\end{array}$ & $\begin{array}{c}\text { Validation } \\
\text { set }^{1}\end{array}$ & Prevalence class & $\begin{array}{c}\text { Training } \\
\text { set }^{1}\end{array}$ & $\begin{array}{c}\text { Validation } \\
\text { set }^{1}\end{array}$ \\
\hline Non-host & $249(54 \%)$ & $257(58 \%)$ & Non-host & $222(67 \%)$ & $228(72 \%)$ \\
\hline Uninfected host & $147(31 \%)$ & 153 (34\%) & Uninfected host & 94 (28\%) & 70 (22\%) \\
\hline Low prevalence & $21(5 \%)$ & $12(3 \%)$ & Low prevalence & $5(1 \%)$ & $6(2 \%)$ \\
\hline Medium prevalence & $21(5 \%)$ & $10(2 \%)$ & Medium prevalence & $6(2 \%)$ & $6(2 \%)$ \\
\hline High prevalence & $21(5 \%)$ & $13(3 \%)$ & High prevalence & $6(2 \%)$ & $5(2 \%)$ \\
\hline TOTAL & 459 & 445 & TOTAL & 333 & 315 \\
\hline
\end{tabular}

${ }^{1}$ Datasets containing information collected during the first sampling cycle (Training set) and the second sampling cycle (Validation set). 


\subsubsection{Complete ML models of genus-wide potyvirus prevalence}

ML models were built using the above-defined classes of potyvirus prevalence as the response variable (Table 4.8). The following ecological factors were considered as predictors of potyvirus prevalence in the studied ecosystems: location, season, plant family, plant species, plant life cycle, plant density and relative abundance, plant biomass, species richness $(\mathrm{S})$, Shannon's index ( $\left.\mathrm{H}^{\prime}\right)$ and Shannon's equitability index $\left(\mathrm{E}_{\mathrm{H}}\right)$, soil $\mathrm{pH}$, minimum, maximum and seasonal averaged values of temperature, relative humidity and rainfalls and number of host plant species in each location (Table 4.9). Three different ML algorithms were run: Multinomial Logistic Regression (Logistic), J48 Decision Tree (J48) and Random Forest. In order to compare ML algorithms, the ZeroR algorithm was also run to represent the baseline for classification accuracy. Classification models were considered valuable if they had better performance than the baseline algorithm (better classification than expected by chance).

Table 4.9. Mean values of continuous variables used as predictors in ML models for potyvirus prevalence in the studied ecosystems.

\begin{tabular}{|c|c|c|c|c|}
\hline \multirow[b]{2}{*}{ Predictors } & \multicolumn{2}{|c|}{ Riparian forests } & \multicolumn{2}{|c|}{ Evergreen oak forests } \\
\hline & Training set & Validation set & Training set & Validation set \\
\hline Plant density (m) & $5.48 \pm 5.24$ & $5.69 \pm 8.64$ & $5.49 \pm 4.95$ & $4.13 \pm 3.51$ \\
\hline $\begin{array}{l}\text { Relative abundance } \\
(\%)\end{array}$ & $3.51 \pm 3.23$ & $3.51 \pm 3.47$ & $4.82 \pm 4.56$ & $5.76 \pm 6.51$ \\
\hline Biomass $\left(m^{3}\right)$ & $0.15 \pm 0.14$ & $0.10 \pm 0.07$ & $0.06 \pm 0.06$ & $0.06 \pm 0.07$ \\
\hline S & $31.00 \pm 3.80$ & $29.17 \pm 6.48$ & $21.91 \pm 5.26$ & $21.30 \pm 4.42$ \\
\hline $\mathrm{H}^{\prime}$ & $3.03 \pm 0.20$ & $2.93 \pm 0.29$ & $2.72 \pm 0.30$ & $2.45 \pm 0.30$ \\
\hline $\mathrm{E}_{\mathrm{H}}$ & $0.89 \pm 0.05$ & $0.86 \pm 0.04$ & $0.88 \pm 0.05$ & $0.81 \pm 0.06$ \\
\hline HPS & $13.50 \pm 3.70$ & $12.38 \pm 3.89$ & $8.50 \pm 1.87$ & $5.86 \pm 2.41$ \\
\hline $\mathrm{T}_{\min }\left({ }^{\circ} \mathrm{C}\right)$ & $3.04 \pm 3.38$ & $4.33 \pm 4.31$ & $3.73 \pm 3.30$ & $5.43 \pm 4.01$ \\
\hline $\mathrm{T}_{\text {med }}\left({ }^{\circ} \mathrm{C}\right)$ & $15.32 \pm 3.57$ & $16.31 \pm 4.00$ & $16.12 \pm 3.62$ & $17.65 \pm 4.27$ \\
\hline $\mathrm{T}_{\max }\left({ }^{\circ} \mathrm{C}\right)$ & $28.20 \pm 3.56$ & $29.71 \pm 3.48$ & $28.15 \pm 3.52$ & $29.71 \pm 4.05$ \\
\hline $\mathrm{RH}_{\min }(\%)$ & $18.37 \pm 4.93$ & $18.49 \pm 5.69$ & $18.92 \pm 5.40$ & $19.00 \pm 6.34$ \\
\hline $\mathrm{RH}_{\text {med }}(\%)$ & $54.21 \pm 8.67$ & $56.56 \pm 10.35$ & $50.33 \pm 8.69$ & $52.08 \pm 13.46$ \\
\hline $\mathrm{RH}_{\max }(\%)$ & $96.50 \pm 3.05$ & $97.24 \pm 1.91$ & $93.78 \pm 4.79$ & $94.50 \pm 5.15$ \\
\hline Rainfalls (mm) & $370.72 \pm 288.85$ & $351.98 \pm 280.93$ & $179.08 \pm 130.50$ & $326.29 \pm 276.86$ \\
\hline Soil pH & $5.25 \pm 0.35$ & $5.25 \pm 0.35$ & $5.33 \pm 0.76$ & $5.33 \pm 0.76$ \\
\hline \multicolumn{5}{|c|}{$\begin{array}{l}\text { Mean } \pm \text { standard deviation of continuous predictor variables in the first sampling cycle (Training set) and the } \\
\text { second sampling cycle (Validation set). S: Species richness; } H^{\prime}: \text { Shannon's index; EH: Shannon's } \\
\text { equitability index; HPS: number of host plants at a given location; } T_{\max }, T_{\min }, T_{\operatorname{mean}} \text { : Average of monthly } \\
\text { maximum, minimum, and mean temperatures at a given season/location; } R H_{\max }, \mathrm{RH}_{\min }, \mathrm{RH} \mathrm{H}_{\operatorname{mean}} \text { : Average } \\
\text { of monthly maximum, minimum, and mean relative humidity at a given season/location; Rainfalls: Average } \\
\text { of total rainfalls in a season. }\end{array}$} \\
\hline
\end{tabular}


Overall performance of the $\mathrm{ML}$ algorithms was evaluated using three measures: the Accuracy, the True Positive rate (TPR) and the area under the ROC curve (AUROC) (see section 3.5.2.1.2). In riparian forests, comparison between ML model performances showed that, in general, all three performed better than the baseline, regardless of cross-validation methods or a validation data set were used (Table 4.10). According to cross-validation methods, Logistic had the worst accuracy and AUROC values (Accuracy $=47 \%$, $A U R O C=72 \%$, and the highest TP rate (TPR=25\%) (Table 4.10). Also, this model had a drop in accuracy when it was challenged against the validation dataset (Accuracy=30\%, AUROC=67\% and TPR=17\%). On the other hand, and according to cross-validation methods, J48 and Random Forest models had better performance than Logistic, except for the TPR (Accuracy $=75 \%$ and $72 \%$, AUROC $=84 \%$ and $74 \%$, and TPR=13\% and 15\%, respectively) (Table 4.10). These two ML models presented more robust values than Logistic when compared with the performance on the validation dataset (Accuracy $=86 \%$ and $81 \%, A U R O C=91 \%$ and $92 \%$, and TPR=11\% and $22 \%$ ). Indeed, J48 and Random forest generally showed better performances on the validation dataset than in cross-validation analyses (Table 4.10). Thus, the built models have good predictive power not only in subsets of data from the same sampling cycle used to construct them (cross validation), but, more importantly, in a different dataset than that used to build the model (validation set).

Table 4.10. Performance measures (\% of successfully classified instances) of ML models to predict potyvirus prevalence in riparian forests.

\begin{tabular}{lcccccccccc}
\hline & \multicolumn{3}{c}{ 5x2CV training set } & & \multicolumn{4}{c}{ Validation set $^{\mathbf{2}}$} \\
\cline { 2 - 5 } \cline { 8 - 10 } & ZeroR & Logistic & J48 & $\begin{array}{c}\text { Random } \\
\text { Forest }\end{array}$ & & ZeroR & Logistic & J48 & $\begin{array}{c}\text { Random } \\
\text { Forest }\end{array}$ \\
\hline Accuracy & 54 & 47 & 75 & 72 & & 58 & 30 & 86 & 81 \\
AUROC & 50 & 72 & 84 & 74 & & 50 & 67 & 91 & 92 \\
TPR & 0 & 25 & 13 & 15 & & 0 & 17 & 11 & 22 \\
\hline
\end{tabular}

${ }^{1}$ Models were evaluated by means of 5 replications of two-fold cross validation $(5 \times 2 \mathrm{CV})$ on the training dataset (first sampling cycle).

${ }^{2}$ Models were challenged with dataset containing information collected during the second sampling cycle. AUROC: Area under the ROC curve (AUROC); TPR: True Positive rate.

Values are shown as percentages.

In evergreen oak forests, the three models showed similar performances than in riparian forests (Table 4.11). The exceptions were: (i) that J48 and 
Random forest did not increase values of the AUROC curve when challenged with the validation dataset. Rather, the AUROC values were similar to those of the ZeroR baseline model (Table 4.11); and (ii) that AUROC values of the Logistic model were equal or better than in the other two models (Table 4.11).

Table 4.11. Performance measures (\% of successfully classified instances) of ML models to predict potyvirus prevalence in evergreen oak forests.

\begin{tabular}{|c|c|c|c|c|c|c|c|c|}
\hline & \multicolumn{4}{|c|}{$5 \times 2 C V$ training set ${ }^{1}$} & \multicolumn{4}{|c|}{ Validation set ${ }^{2}$} \\
\hline & ZeroR & Logistic & J48 & $\begin{array}{c}\text { Random } \\
\text { Forest }\end{array}$ & ZeroR & Logistic & J48 & $\begin{array}{c}\text { Random } \\
\text { Forest }\end{array}$ \\
\hline Accuracy & 67 & 73 & 75 & 85 & 72 & 60 & 94 & 92 \\
\hline AUROC & 50 & 89 & 75 & 92 & 50 & 80 & 51 & 65 \\
\hline TPR & 0 & 19 & 11 & 17 & 0 & 31 & 20 & 20 \\
\hline
\end{tabular}

${ }^{1}$ Models were evaluated by means of 5 replications of two-fold cross validation $(5 \times 2 \mathrm{CV})$ on the training dataset (first sampling cycle).

${ }^{2}$ Models were challenged with dataset containing information collected during the second sampling cycle. AUROC: Area under the ROC curve (AUROC); TPR: True Positive rate.

Values are shown as percentages.

In the analyses above, all predictor variables were kept in the model (complete model). However, it would be possible that not all predictors have the same relative importance in the accuracy of the model, such that simpler models may have similar or even better performance than the complete model. To test this possibility, different approaches were performed depending on the ML model:

(i) For Logistic models, a stepwise model selection approach was used to compare the accuracy of models built using all possible combinations of the predictors. The best-ranked model explaining potyvirus prevalence in riparian forests contained plant species, plant relative abundance and season as predictors (Table 4.12). This model explained $95 \%$ of the variance in the response variable, plant species having much higher relative importance (98\%) than the other two predictors (Table 4.12). The best model in evergreen oak forests contained plant species, averaged rainfalls, averaged relative humidity and plant biomass. This model explained $99 \%$ of the variance, plant species having much higher relative importance than other predictors (96\%) (Table 4.12). Thus, results indicated that plant identity was the most relevant factor in predicting potyvirus prevalence. Indeed, models in which plant species was the only predictor did not perform worse than the best-ranked model (Table 4.12). Also, the analysis of the predictive power of these models indicated that, 
overall, their accuracy, AUROC and TPR values were equally good or even higher than in the complete models (compare Table 4.10, 4.11 and 4.12), although it should be noted that these models performed worse in the validation dataset than in the training dataset (Table 4.12). In both ecosystems, no other combination of factors yielded a model that closely competed with the bestranked one (Appendix 3, Table A3.1).

4.12. Model fitting and performance of ML models for predicting potyvirus prevalence.

\begin{tabular}{|c|c|c|c|c|c|c|c|c|}
\hline \multirow[t]{2}{*}{ Model structure* } & \multicolumn{2}{|c|}{$\begin{array}{l}\text { Model } \\
\text { fitting } 1\end{array}$} & \multicolumn{3}{|c|}{$\begin{array}{l}\text { Model performance } \\
\text { on } 5 \times 2 \mathrm{CV} \text { training set }{ }^{2}\end{array}$} & \multicolumn{3}{|c|}{$\begin{array}{c}\text { Model performance } \\
\text { on validation set }{ }^{2}\end{array}$} \\
\hline & AIC & $\mathbf{R}^{2} \mathrm{~N}$ & Acc & AUROC & TPR & Acc & AUROC & TPR \\
\hline \multicolumn{9}{|l|}{ Riparian forests } \\
\hline PSP (98)+PRA (1)+SEA (1) & 1407.29 & 0.95 & 92 & 97 & 54 & 83 & 72 & 15 \\
\hline PSP (100) & 1446.74 & 0.93 & 87 & 94 & 25 & 86 & 67 & 12 \\
\hline \multicolumn{9}{|l|}{ Evergreen oak forests } \\
\hline$P S P(96)+R A F(2)+R H_{\text {med }}(1)+V(1)$ & 906.96 & 0.99 & 95 & 98 & 26 & 91 & 70 & 20 \\
\hline PSP (100) & 921.93 & 0.95 & 96 & 97 & 27 & 94 & 68 & 20 \\
\hline
\end{tabular}

${ }^{*}$ The relative importance (\%) of each predictor variable is shown in parenthesis.

${ }^{1}$ Model fitting measures: Akaike's Information Criterion (AIC) and Nagelkerke $R^{2}\left(R^{2} N\right)$.

${ }^{2}$ Model performance measures: rate of correct predictions of the model (Acc: Accuracy), Area under the ROC curve (AUROC), True Positive rate (TPR). Values are shown as percentages.

PSP: Plant species; PRA: Plant relative abundance; SEA: Season; RAF: Rainfalls; $\mathrm{RH}_{\text {med: }}$ Average relative humidity; V: Plant biomass.

(ii) Models obtained with the J48 method were also analysed. J48 methods create a single decision tree from the training dataset. The tree represents the classification rules for the prediction of potyvirus prevalence and includes a root node from where the tree starts, and internal nodes connected by two or more branches. To which of these branches a given observation is assigned depends on threshold values of the predictor defining the node (see Figure 4.11). The tip nodes are known as leaf nodes, and the probability of being assigned to a given potyvirus prevalence category according to the predictors and threshold values that define the tree.

Figure 4.11 illustrates the decision tree for potyvirus prevalence classes in riparian forests, which summarizes the best predictors such that the deeper the node in the tree defined by a given predictor, and the higher the number of nodes defined by that predictor, the higher its relative importance. The trained 
model classified correctly $91 \%, 90 \%$ and $92 \%$ of infected hosts with low, medium and high prevalence, respectively (Figure 4.11). Also, the model correctly assigned $100 \%$ of non-hosts and $93 \%$ of uninfected hosts. Of the predictors evaluated, J48 identified plant species as the best discriminator between prevalence classes (root node) (Figure 4.11). Moreover, presence/absence of certain host species was also identified as a relevant determinant of potyvirus incidence. For instance, Conopodium subcarneum (73\%) and Trifolium pratense (25\%) defined infected hosts with low prevalence, Dactylis glomerata (97\%) defined host with medium prevalence, and Lamium album (90\%) defined hosts species with high prevalence. Plant relative abundance was a relevant predictor of high virus prevalence when Humulus lupulus was the infected host, and its relative abundance was above $1.59 \%$

(Figure 4.11).

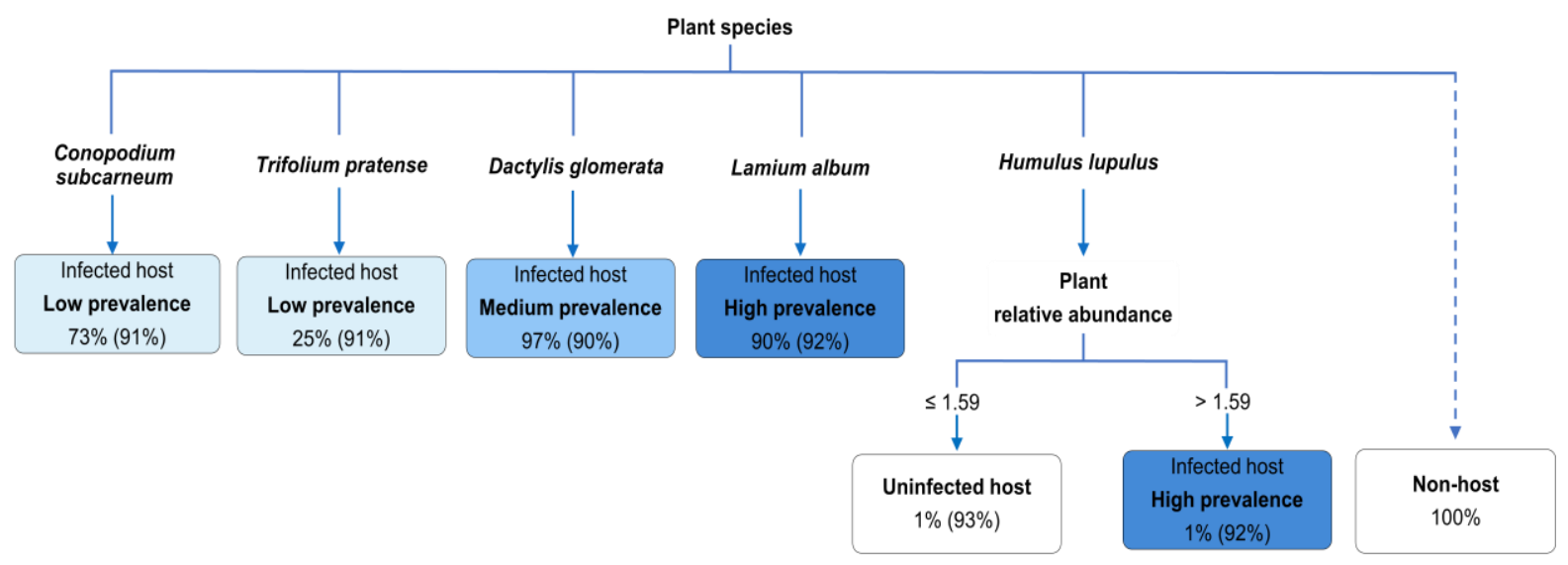

Figure 4.11. Condensed J48 Decision Tree of potyvirus prevalence in riparian forests. Defining predictors and categories (for categorical predictors) or threshold values (for continuous predictors) of each node are represented. Leaf nodes indicate the predicted prevalence class, the percentage of the total number of instances assigned to that prevalence class (in parenthesis) and the percentage of instances assigned to that prevalence class at the specific leaf node (out of the parenthesis). Dashed line group plant species that identified non-hosts that have been collapsed in the tree.

The $\mathrm{J} 48$ decision tree for potyvirus prevalence in evergreen oak forests training correctly classified $99 \%$ of uninfected host and non-hosts, $94 \%$ of infected hosts with low and medium prevalence and $98 \%$ of hosts with high prevalence (Figure 4.12). Again, this model identified plant species as the best predictor of prevalence class. (Leontodon longirostris defined all instances 
classified as infected hosts with low prevalence from Marjaliza. Also, Tolpis umbellata defined infected hosts with medium prevalence when minimum relative humidity was higher than $15 \%$. Finally, Ruta montana defined most cases of infected hosts with high prevalence (Figure 4.12).

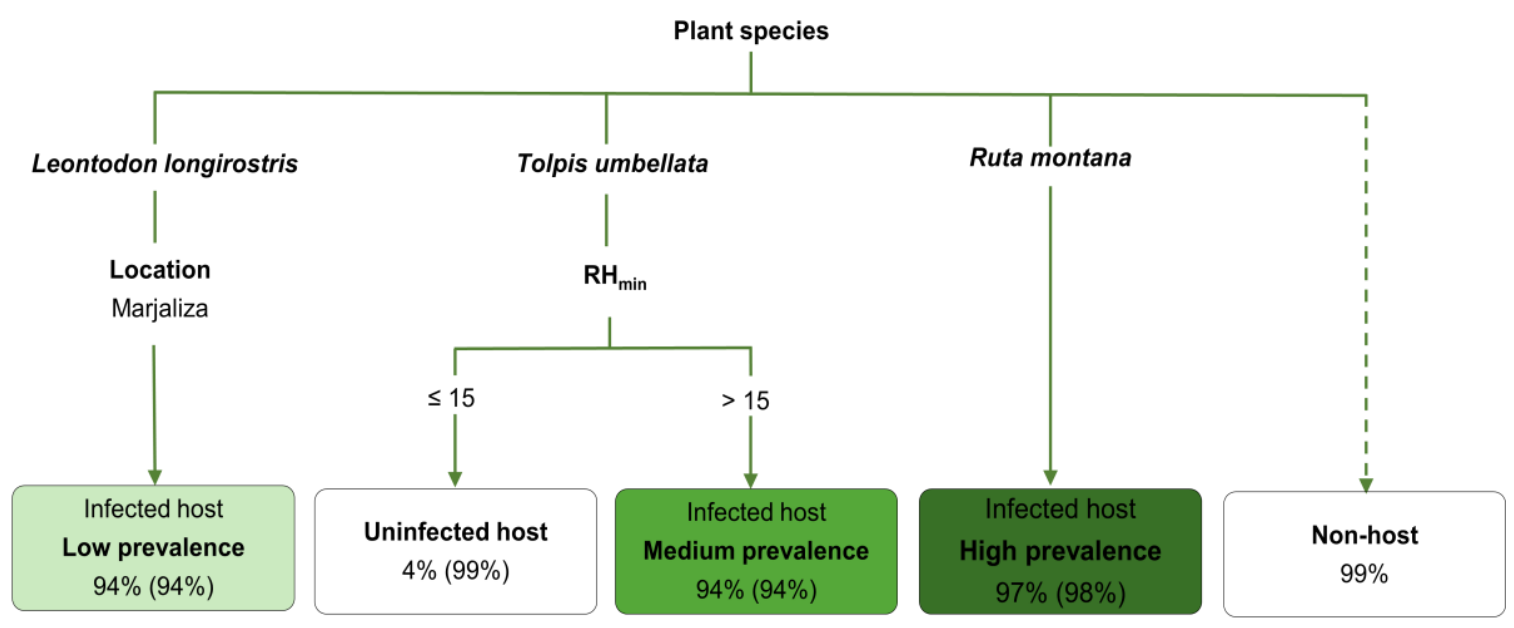

Figure 4.12. Condensed J48 Decision Tree of potyvirus prevalence in evergreen oak forests. Defining predictors and categories (for categorical predictors) or threshold values (for continuous predictors) of each node are represented. Leaf nodes indicate the predicted prevalence class, the percentage of the total number of instances assigned to that prevalence class (in parenthesis) and the percentage of instances assigned to that prevalence class at the specific leaf node (out of the parenthesis). Dash line represents rest of classification rules that were associated to plant species. Dashed line group plant species that identified non-hosts that have been collapsed in the tree.

(iii) As mentioned in section 3.5.2.1.2, J48 Decision Trees provide less robust results that Random Forest models. Thus, to confirm the results obtained with $\mathrm{J48}$ and Logistic models, the relative importance of each predictor in determining potyvirus prevalence was analysed by Random Forest models and ranked based on Mean Decrease Impurity (MDI) measures. In both ecosystems, plant family and species were the most relevant predictors (MDI>0.75) of potyvirus prevalence (Figure 4.13). The remaining predictors had similar relative importance (MDI=0.35-0.5) in both ecosystems (Figure 4.13). 

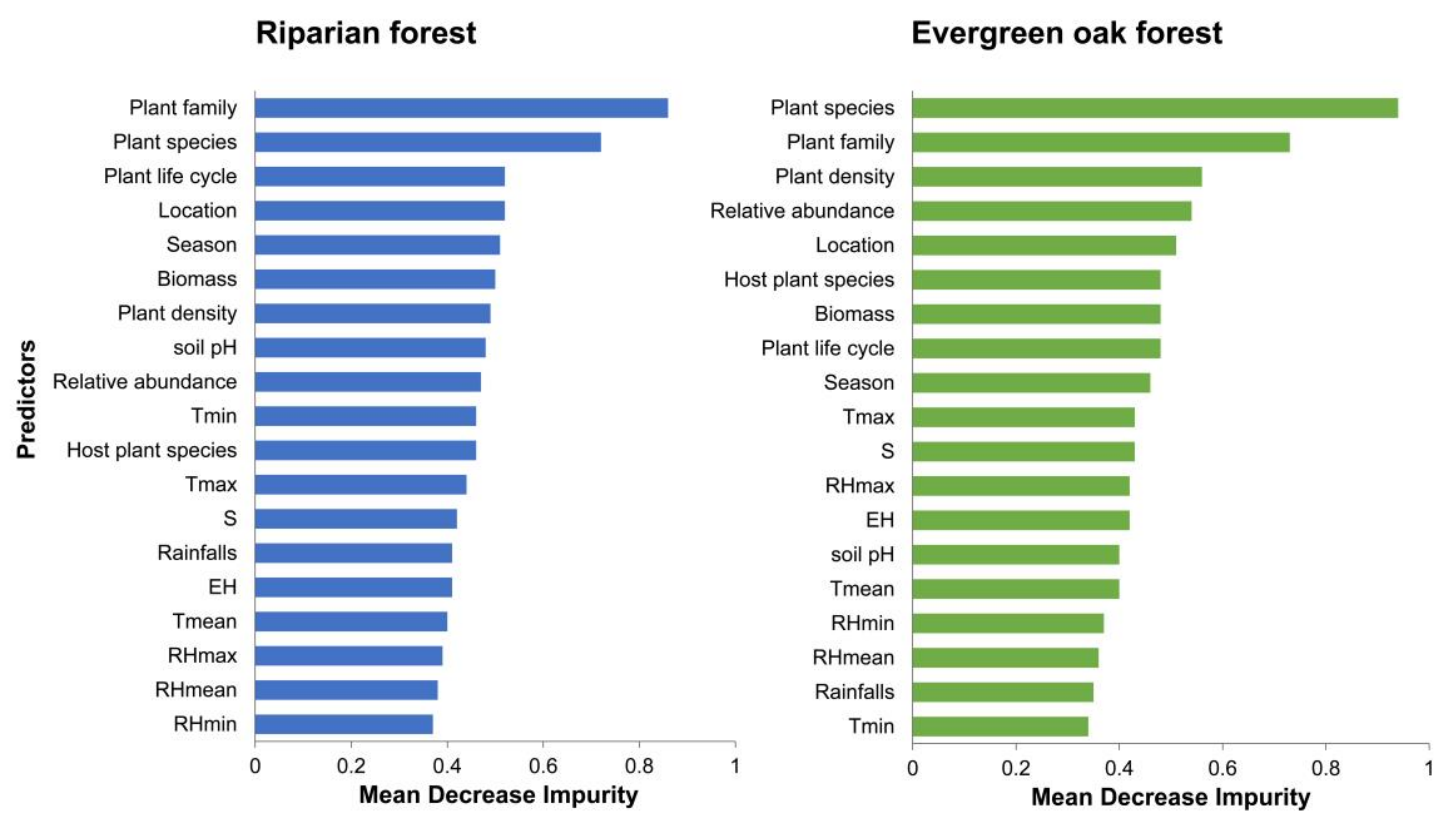

Figure 4.13. Relative importance of the predictors of potyvirus prevalence according to Mean Decrease Impurity (MDI) values from 100 Random Forest trees. Trees were generated for the riparian forests (blue) and evergreen oak forests (green) datasets. Values are normalized in a range from 0 to 1 . The higher the MDI of a predictor, the more relevant the predictor is.

In summary, the utilized ML methods constructed models with high accuracy, in both the training and the validation data sets. These models detected the identity of the plant species present in an ecosystem as the major (and often the unique) predictor of potyvirus prevalence, regardless of the ML method used. The dominance of plant species identity as a predictor of potyvirus prevalence could mask the effect of other factors that may also be important for virus prevalence. Thus, ML models were constructed excluding the predictors associated with plant identity (plant species and plant family).

\subsubsection{ML models of genus-wide potyvirus prevalence excluding plant identity}

The performance of the Logistic, J48 and Random Forest methods was assessed as in the previous section. Comparison between model performances showed that in both ecosystems, the three ML methods had performance scores close or even lower than the baseline (ZeroR) model in both the training and the validation datasets (Table 4.13 and 4.14). 
Table 4.13. Model fitting and performance of ML models for predicting potyvirus prevalence in riparian forests excluding plant identity.

\begin{tabular}{|c|c|c|c|c|c|c|c|c|}
\hline \multicolumn{5}{|c|}{$5 \times 2 C V$ training set ${ }^{1}$} & \multicolumn{4}{|c|}{ Validation set ${ }^{2}$} \\
\hline & ZeroR & Logistic & J48 & $\begin{array}{c}\text { Random } \\
\text { Forest }\end{array}$ & ZeroR & Logistic & J48 & $\begin{array}{c}\text { Random } \\
\text { Forest }\end{array}$ \\
\hline Accuracy & 54 & 47 & 46 & 47 & 58 & 42 & 42 & 41 \\
\hline AUROC & 50 & 61 & 57 & 59 & 50 & 60 & 58 & 59 \\
\hline TPR & 0 & 28 & 26 & 26 & 0 & 40 & 38 & 34 \\
\hline
\end{tabular}

${ }^{1}$ Models were evaluated by means of 5 replications of two-fold cross validation $(5 \times 2 \mathrm{CV})$ on the training dataset (first sampling cycle).

${ }^{2}$ Models were challenged with dataset containing information collected during the second sampling cycle.

AUROC: Area under the ROC curve (AUROC); TPR: True Positive rate.

Values are shown as percentages.

Table 4.14. Model fitting and performance of ML models for predicting potyvirus prevalence in evergreen oak forests excluding plant identity.

\begin{tabular}{|c|c|c|c|c|c|c|c|c|}
\hline \multicolumn{5}{|c|}{$5 \times 2 C V$ training set ${ }^{1}$} & \multicolumn{4}{|c|}{ Validation set ${ }^{2}$} \\
\hline & ZeroR & Logistic & J48 & $\begin{array}{c}\text { Random } \\
\text { Forest }\end{array}$ & ZeroR & Logistic & J48 & $\begin{array}{c}\text { Random } \\
\text { Forest }\end{array}$ \\
\hline Accuracy & 67 & 62 & 60 & 58 & 72 & 17 & 39 & 61 \\
\hline AUROC & 50 & 58 & 54 & 58 & 50 & 47 & 51 & 65 \\
\hline TPR & 0 & 5 & 6 & 0 & 0 & 19 & 34 & 30 \\
\hline
\end{tabular}

${ }^{1}$ Models were evaluated by means of 5 replications of two-fold cross validation $(5 \times 2 \mathrm{CV})$ on the training dataset (first sampling cycle).

${ }^{2}$ Models were challenged with dataset containing information collected during the second sampling cycle. AUROC: Area under the ROC curve (AUROC); TPR: True Positive rate.

Values are shown as percentages.

The relative importance of the predictors considered by these models were also analysed:

(i) Using model selection method for Logistic models, in riparian forests the best-ranked models had Accuracy and TPR values that were not significantly higher than those of the complete models, were validated with the training or the validation datasets (55\% and $5 \%$ vs. $58 \%$ and $3 \%)$. However, AUROC values were significantly higher in the simpler than in the complete model $(72 \%$ vs. $61 \%$ and $72 \%$ vs. $60 \%$, for the training or the validation datasets, respectively), indicating better predictive power (Table 4.15). The best-ranked model explained $24 \%$ of the variance in the potyvirus prevalence in riparian forests (Table 4.15). Similar results were obtained in evergreen oak forests. Also, the most relevant ecological factors identified in Logistic models were largely the same in both ecosystems. Plant relative abundance had the highest relative importance $(46 \%$ and $80 \%$ in riparian and evergreen oak forests, respectively), and plant life cycle was the second most important factors 
(relative importance: $25 \%$ and $20 \%$ in riparian and evergreen oak forests, respectively). In riparian forests, the number of host species (relative importance: $21 \%$ ) and rainfalls (relative importance: $8 \%$ ) were also included in the models (Table 4.15). In riparian forest, a logistic model including the same factors as the best-raked one but excluding rainfalls had similar predictive power (Appendix 3, Table A3.2). In evergreen oak forests, no other model closely competed with the best ranked one (Appendix 3, Table A3.2).

Table 4.15. Model fitting and performance of $M L$ models for predicting potyvirus prevalence excluding plant identity.

\begin{tabular}{|c|c|c|c|c|c|c|c|c|}
\hline \multirow[t]{2}{*}{ Model structure } & \multicolumn{2}{|c|}{$\begin{array}{l}\text { Model } \\
\text { fitting } 1\end{array}$} & \multicolumn{3}{|c|}{$\begin{array}{l}\text { Model performance } \\
\text { on } 5 \times 2 \mathrm{CV} \text { training set }{ }^{2}\end{array}$} & \multicolumn{3}{|c|}{$\begin{array}{c}\text { Model performance } \\
\text { on validation set }\end{array}$} \\
\hline & AIC & $\mathbf{R}^{2}{ }_{\mathrm{N}}$ & Acc & AUROC & TPR & Acc & AUROC & TPR \\
\hline \multicolumn{9}{|l|}{ Riparian forests } \\
\hline PRA (46)+PLC (25)+HPS (21)+RAF (8) & 914.75 & 0.24 & 55 & 72 & 5 & 58 & 72 & 3 \\
\hline \multicolumn{9}{|l|}{ Evergreen oak forests } \\
\hline PRA (80)+PLC (20) & 516.05 & 0.15 & 67 & 71 & 0 & 62 & 60 & 0 \\
\hline
\end{tabular}

(ii) J48 models generated large and complex decision trees. Because of the difficulty in summarizing this tree, only the raw tree is provided in Appendix 3, Figures A3.1 and A3.2. In riparian forests, plant relative abundance was the predictor with the highest relative importance, as it was located in the root node and defined the highest number of nodes in the tree (36 out of 164 nodes). Plant density and number of host plant species were also relevant predictors, as they defined the same number of nodes in the tree than plant relative abundance (36 out of 164 nodes) (Appendix 3, Figure A3.1). Other ecological factors also appeared in the tree (season, plant life cycle, species richness, plant biomass, $\mathrm{E}_{\mathrm{H}}, \mathrm{T}_{\mathrm{mean}}, \mathrm{T}_{\mathrm{max}}, \mathrm{RH}$ min, and rainfalls), but defined a small number of nodes (less than 10 out of 164 each) (Appendix 3, Figure A3.1). In evergreen oak forests, season was the predictor defining the root node (Appendix 3, Figure A3.2). Plant density (defining 16 out of 64 nodes), and plant relative abundance (12 out of 64 nodes) were also important predictors of potyvirus prevalence as they defined the largest number of nodes (Appendix 3, 
Figure A3.2). (Appendix, Figure A3.2). Other factors that appeared in the tree were plant life cycle, number of host plant species, plant biomass, $\mathrm{E}_{\mathrm{H}}, \mathrm{T}_{\min }$ and $\mathrm{RH}_{\max }$, with low relative importance (defined less than 5 out of 64 nodes each).

(iii) Finally, according to Random Forest methods the predictors with the higher relative importance (higher MDI values) were plant density and relative abundance $(\mathrm{MDI}>0.5)$. All other predictors presented MDI values lower than 0.4 (Figure 4.14). Note that plant life cycle, one of the predictors with higher relative importance in Logistic models, was also classified among the top five predictors of potyvirus prevalence by Random Forest methods.

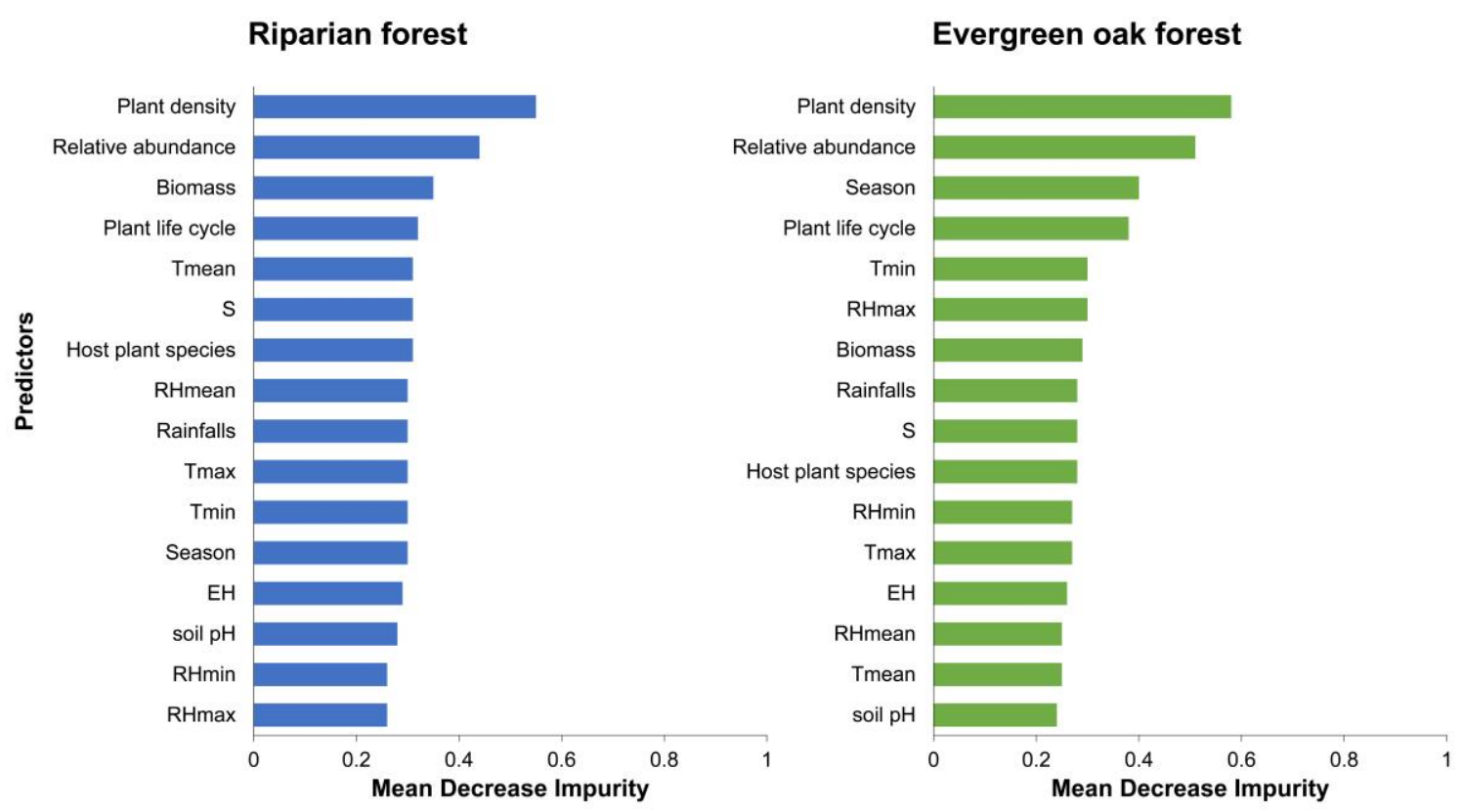

Figure 4.14. Relative importance of the predictors of potyvirus prevalence according to Mean Decrease Impurity (MDI) values from 100 Random Forest trees. Trees were generated for the riparian forests (blue) and evergreen oak forests (green) datasets, excluding plant identity traits as predictors. Values are normalized in a range from 0 to 1 . The higher the MDI of a predictor, the more relevant the predictor is.

In summary, excluding plant identity traits reduced the accuracy of ML models in predicting potyvirus prevalence, but allowed identifying other ecological factors that also affect such prevalence. All three ML models identified plant relative abundance as a predictor, and most models also 
indicated that plant density and plant life cycle (annual or perennial) have a role in determining potyvirus prevalence in riparian and evergreen oak forests.

\subsubsection{Role of $M L$ predictors in determining genus-wide potyvirus prevalence}

Results obtained in sections 4.2.2.1 and 4.2.2.2 showed that ML models with the best predictive power contained plant species as the most important predictor. These models predicted mostly non-host and uninfected hosts categories (high Accuracy and AUROC), but showed lower predictive power for infected hosts with low, medium and high prevalence (low TPR). Interestingly, although ML models excluding plant identity traits had lower Accuracy and AUROC, had higher TPR values. These results suggested that: (i) complete ML models, particularly when plant species identity is considered, could be more powerful to predict presence/absence of potyvirus infection; and (ii) factors other than plant identity may be relevant to predict the intensity of potyvirus infection (i.e., the level of prevalence). In order to investigate these questions, models that contained plant identity as unique predictor or a combination of host relative abundance, plant density were constructed to predict the two major classes: non-host and host plant species.

\subsection{ML models of genus-wide potyvirus presence/absence}

To analyse the ecological factors affecting the presence/absence of potyviruses in the studied ecosystems, the categories of virus prevalence were simplified into two categories: Non-hosts, which constituted again the majority class representing between $54 \%-72 \%$ of the observations, and host plant species (the sum of uninfected and infected hosts), which represented between $28 \%$ $46 \%$ of the observations (Table 4.8 ).

In riparian forests, the three $M L$ models showed robust performance in both cross-validation and validation analyses. Logistic model had lower accuracy when it was challenged against the validation set than in crossvalidation analyses (Accuracy $=76 \%$ and $94 \%, A U R O C=84 \%$ and $98 \%$, respectively). J48 and Random Forest showed similar or better performances on the validation dataset than in cross-validation analyses (Accuracy $=98 \%$ and 
97\%; $\mathrm{AUROC}=99 \%$ and $99 \%$ and Accuracy=92\% and $93 \%, \mathrm{AUROC}=99 \%$ and 98\%, respectively) (Table 4.16).

Table 4.16. Performance measures (\% of successfully classified instances) of ML models to predict host species in riparian forests.

\begin{tabular}{|c|c|c|c|c|c|c|c|c|}
\hline \multicolumn{5}{|c|}{$5 \times 2 \mathrm{CV}$ training set ${ }^{1}$} & \multicolumn{4}{|c|}{ Validation set ${ }^{2}$} \\
\hline & ZeroR & Logistic & J48 & $\begin{array}{c}\text { Random } \\
\text { Forest }\end{array}$ & ZeroR & Logistic & J48 & $\begin{array}{c}\text { Random } \\
\text { Forest }\end{array}$ \\
\hline Accuracy & 54 & 94 & 97 & 93 & 55 & 76 & 98 & 92 \\
\hline AUROC & 50 & 98 & 99 & 98 & 50 & 84 & 99 & 99 \\
\hline TPR & 54 & 94 & 97 & 93 & 55 & 76 & 98 & 92 \\
\hline
\end{tabular}

${ }^{1}$ Models were evaluated by means of 5 replications of two-fold cross validation $(5 \times 2 \mathrm{CV})$ on the training dataset (first sampling cycle).

${ }^{2}$ Models were challenged with dataset containing information collected during the second sampling cycle.

AUROC: Area under the ROC curve (AUROC); TPR: True Positive rate.

Values are shown as percentages.

In evergreen oak forests, the three models showed similar performance scores than in riparian forests: Logistic model had a high predictive power that was worse in validation than in cross-validation analyses (Accuracy $=70 \%$ and 97\%, AUROC=92\% and 99\%, respectively) (Table 4.17). Although J48 performance values were similar to those of the ZeroR baseline model in crossvalidation analyses, predictive power was maximal when this model was challenged against the validation set (Accuracy $=67 \%$ and $100 \%, A U R O C=49 \%$ and $100 \%$, respectively). Random Forest models showed very high predictive power, and similar or better performances on the validation dataset than in cross-validation analyses (Table 4.17).

Table 4.17. Performance measures (\% of successfully classified instances) of ML models to predict host species in evergreen oak forests.

\begin{tabular}{|c|c|c|c|c|c|c|c|c|}
\hline \multicolumn{5}{|c|}{$5 \times 2 C V$ training set ${ }^{1}$} & \multicolumn{4}{|c|}{ Validation set ${ }^{2}$} \\
\hline & ZeroR & Logistic & J48 & $\begin{array}{c}\text { Random } \\
\text { Forest }\end{array}$ & ZeroR & Logistic & J48 & $\begin{array}{c}\text { Random } \\
\text { Forest }\end{array}$ \\
\hline Accuracy & 67 & 97 & 67 & 97 & 72 & 70 & 100 & 99 \\
\hline AUROC & 49 & 99 & 49 & 99 & 50 & 92 & 100 & 100 \\
\hline TPR & 67 & 97 & 67 & 97 & 72 & 70 & 100 & 99 \\
\hline
\end{tabular}

${ }^{1}$ Models were evaluated by means of 5 replications of two-fold cross validation $(5 \times 2 \mathrm{CV})$ on the training dataset (first sampling cycle).

2 Models were challenged with dataset containing information collected during the second sampling cycle. AUROC: Area under the ROC curve (AUROC); TPR: True Positive rate.

Values are shown as percentages. 
Remarkably, the high performance of the ML models predicting presence/absence of potyvirus prevalence was achieved by including plant species identity as the sole predictor, with no other ecological factor being relevant in predicting virus prevalence. Thus, the species present in a given ecosystem determine the likelihood of potviruses to enter in such ecosystem.

\subsection{ML models of genus-wide potyvirus prevalence level}

$\mathrm{ML}$ models considering only observations in the low, medium or high potyvirus prevalence classes were constructed. Note that here TPR and accuracy represent the same measure, and thus have the same values.

In riparian forests, comparison between ML model performances showed that, all three performed better than the baseline, regardless of cross-validation method or a validation data set were used (Table 4.18). According to crossvalidation methods, Random Forest had the best accuracy and AUROC values (Accuracy $=68 \%, A U R O C=82 \%$ ). This model had worse predictive power when it was challenged against the validation dataset (Accuracy $=63 \%$, AUROC $=67 \%$ ). Logistic and $J 48$ models had similar performance measures in cross-validation analyses (Accuracy $=63 \%, A \cup R O C=76 \%$ and Accuracy $=60 \%$, AUROC $=75 \%$, respectively). These models also had a drop in accuracy when they were challenged against the validation dataset (Table 4.18).

Table 4.18. Performance measures (\% of successfully classified instances) of ML models to predict host potyvirus prevalence in riparian forests.

\begin{tabular}{|c|c|c|c|c|c|c|c|c|}
\hline \multicolumn{5}{|c|}{$5 \times 2 \mathrm{CV}$ training set ${ }^{1}$} & \multicolumn{4}{|c|}{ Validation set ${ }^{2}$} \\
\hline & ZeroR & Logistic & J48 & $\begin{array}{c}\text { Random } \\
\text { Forest }\end{array}$ & ZeroR & Logistic & J48 & $\begin{array}{c}\text { Random } \\
\text { Forest }\end{array}$ \\
\hline Accuracy & 32 & 63 & 60 & 68 & 34 & 46 & 63 & 63 \\
\hline AUROC & 46 & 76 & 75 & 82 & 50 & 66 & 66 & 67 \\
\hline TPR & 32 & 64 & 60 & 68 & 34 & 46 & 63 & 63 \\
\hline
\end{tabular}

${ }^{1}$ Models were evaluated by means of 5 replications of two-fold cross validation $(5 \times 2 \mathrm{CV})$ on the training dataset (first sampling cycle).

${ }_{2}$ Models were challenged with dataset containing information collected during the second sampling cycle. AUROC: Area under the ROC curve (AUROC); TPR: True Positive rate.

Values are shown as percentages.

In general, ML models had low predictive power in evergreen oak forests when cross-validation methods were used (Table 4.19). However, all three models performed better than the baseline, Random Forests also having the best performance in cross-validation analysis (Accuracy $=35 \%$, 
AUROC $=43 \%$ ). In this case, the predictive power was significantly better when the model was challenged against the validation dataset (Accuracy $=76 \%$, AUROC=91\%). Logistic and J48 models had low performance scores in crossvalidation analyses but their predictive power was higher when the validation dataset was used (Accuracy $=29 \%$ and $41 \%, \quad A U R O C=50 \%$ and $65 \%$; Accuracy $=24 \%$ and $65 \%, A U R O C=37 \%$ and $79 \%$, respectively).

Table 4.19. Performance measures (\% of successfully classified instances) of ML models to predict host potyvirus prevalence in evergreen oak forests.

\begin{tabular}{|c|c|c|c|c|c|c|c|c|}
\hline \multicolumn{5}{|c|}{$5 \times 2 C V$ training set ${ }^{1}$} & \multicolumn{4}{|c|}{ Validation set ${ }^{2}$} \\
\hline & ZeroR & Logistic & J48 & $\begin{array}{c}\text { Random } \\
\text { Forest }\end{array}$ & ZeroR & Logistic & J48 & $\begin{array}{c}\text { Random } \\
\text { Forest }\end{array}$ \\
\hline Accuracy & 12 & 29 & 24 & 35 & 35 & 41 & 65 & 76 \\
\hline AUROC & 18 & 50 & 37 & 43 & 50 & 65 & 79 & 91 \\
\hline TPR & 12 & 29 & 24 & 35 & 35 & 41 & 65 & 77 \\
\hline
\end{tabular}

${ }^{1}$ Models were evaluated by means of 5 replications of two-fold cross validation $(5 \times 2 \mathrm{CV})$ on the training dataset (first sampling cycle).

2 Models were challenged with dataset containing information collected during the second sampling cycle. AUROC: Area under the ROC curve (AUROC); TPR: True Positive rate.

Values are shown as percentages.

As in previous sections, the relative importance of the predictors considered by each model were studied:

(i) Using model selection method for Logistic models, in riparian forests the best-ranked model explained $85 \%$ of the variance, plant relative abundance having higher relative importance $(67 \%)$ than the other predictors in the model: rainfalls, species richness, season, host plant density and plant biomass, which had relative importances lower than 10\% (Table 4.20). In evergreen oak forests, the best-ranked model explained $99 \%$ of the variance. This model contained four predictors: number of host plant species had higher relative importance (32\%), followed by plant relative abundance $(26 \%)$ and host plant density (25\%), and finally rainfalls (17\%) (Table 4.20). No other model closely competed with the best-ranked ones (Appendix 3, Table A3.3). Regardless of the ecosystem, the Accuracy and AUROC values of these models were higher than those of the complete models that excluded plant identity traits (compare Tables 4.13 and 4.14 with Tables 4.18 and 4.19). 
4.20. Model fitting and performance of ML models for predicting host potyvirus prevalence.

\begin{tabular}{|c|c|c|c|c|c|c|c|c|}
\hline \multirow[t]{2}{*}{ Model structure* } & \multicolumn{2}{|c|}{$\begin{array}{l}\text { Model } \\
\text { fitting } 1\end{array}$} & \multicolumn{3}{|c|}{$\begin{array}{l}\text { Model performance } \\
\text { on } 5 \times 2 \mathrm{CV} \text { training set }{ }^{2}\end{array}$} & \multicolumn{3}{|c|}{$\begin{array}{l}\text { Model performance } \\
\text { on validation set }{ }^{2}\end{array}$} \\
\hline & AIC & $\mathbf{R}^{2} \mathrm{~N}$ & Acc & AUROC & TPR & Acc & AUROC & TPR \\
\hline \multicolumn{9}{|l|}{ Riparian forests } \\
\hline PRA (67)+RAF (9)+S (7)+SEA (7)+HPD (6)+V(4) & 79.71 & 0.85 & 75 & 91 & 75 & 71 & 84 & 70 \\
\hline \multicolumn{9}{|l|}{ Evergreen oak forests } \\
\hline HPS (32)+PRA (26)+HPD (25)+RAF(17) & 20.06 & 0.99 & 94 & 99 & 93 & 70 & 83 & 69 \\
\hline \multicolumn{9}{|c|}{$\begin{array}{l}{ }^{*} \text { The relative importance (\%) of each predictor variable is shown in parenthesis. } \\
1 \text { Model fitting measures: Akaike's Information Criterion }(A I C) \text { and Nagelkerke } R^{2}\left(R^{2} N\right) \text {. } \\
2 \text { Model performance measures: rate of correct predictions of the model (Acc: Accuracy), Area under the ROC } \\
\text { curve (AUROC), True Positive rate (TPR). Values are shown as percentages. } \\
\text { PRA: Plant relative abundance; RAF: Rainfalls; S: Species richness; SEA: Season; HPD: Host plant density; } \\
\text { V: Plant biomass; HPS: number of host plant species. }\end{array}$} \\
\hline
\end{tabular}

(ii) Figure 4.15 illustrates the $\mathrm{J} 48$ decision tree for host potyvirus prevalence classes in riparian forests. The model classified correctly $67 \%, 90 \%$ and $95 \%$ of infected hosts with low, medium and high prevalence, respectively (Figure 4.15). The first major predictor in the decision tree was plant relative abundance (root node). The same predictor, but with different threshold values, also defined $55 \%$ and $63 \%$ of infected hosts with high and medium prevalence, respectively (Figure 4.15). Plant density and $T_{\max }$ were also important predictors in the decision tree: $\mathrm{T}_{\max }$ defined $45 \%$ of infected hosts with high prevalence, and plant density defined $100 \%$ and $37 \%$ of infected hosts with low and medium prevalence, respectively.

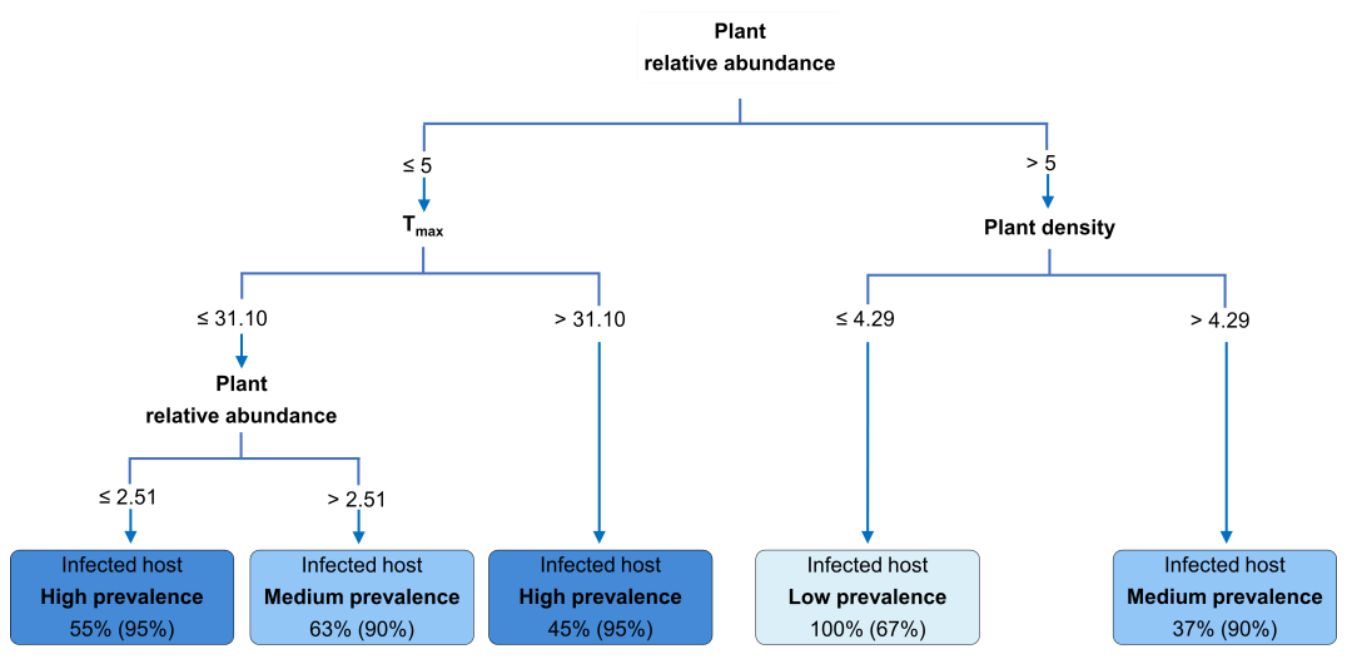

Figure 4.15. Condensed J48 Decision Tree of host potyvirus prevalence in riparian forests. Defining predictors and categories (for categorical predictors) or threshold values (for continuous predictors) of each node are represented. Leaf nodes indicate the predicted prevalence class, the percentage of the total number of instances assigned to that prevalence class (in parenthesis) and the percentage of instances assigned to that prevalence class at the specific leaf node (out of the parenthesis). 
In evergreen oak forests, plant relative abundance was also the most relevant predictor in the $\mathrm{J} 48$ decision tree for host potyvirus prevalence classes (Figure 4.16), followed by plant density, which defined between $40-60 \%$ and $67 \%$ of infected hosts with high and medium prevalence, depending on threshold values (Figure 4.16). Finally, plant life cycle defined 100\% of infected hosts with low prevalence when plants were annuals and $33 \%$ of hosts with medium prevalence when plants were perennials (Figure 4.16).

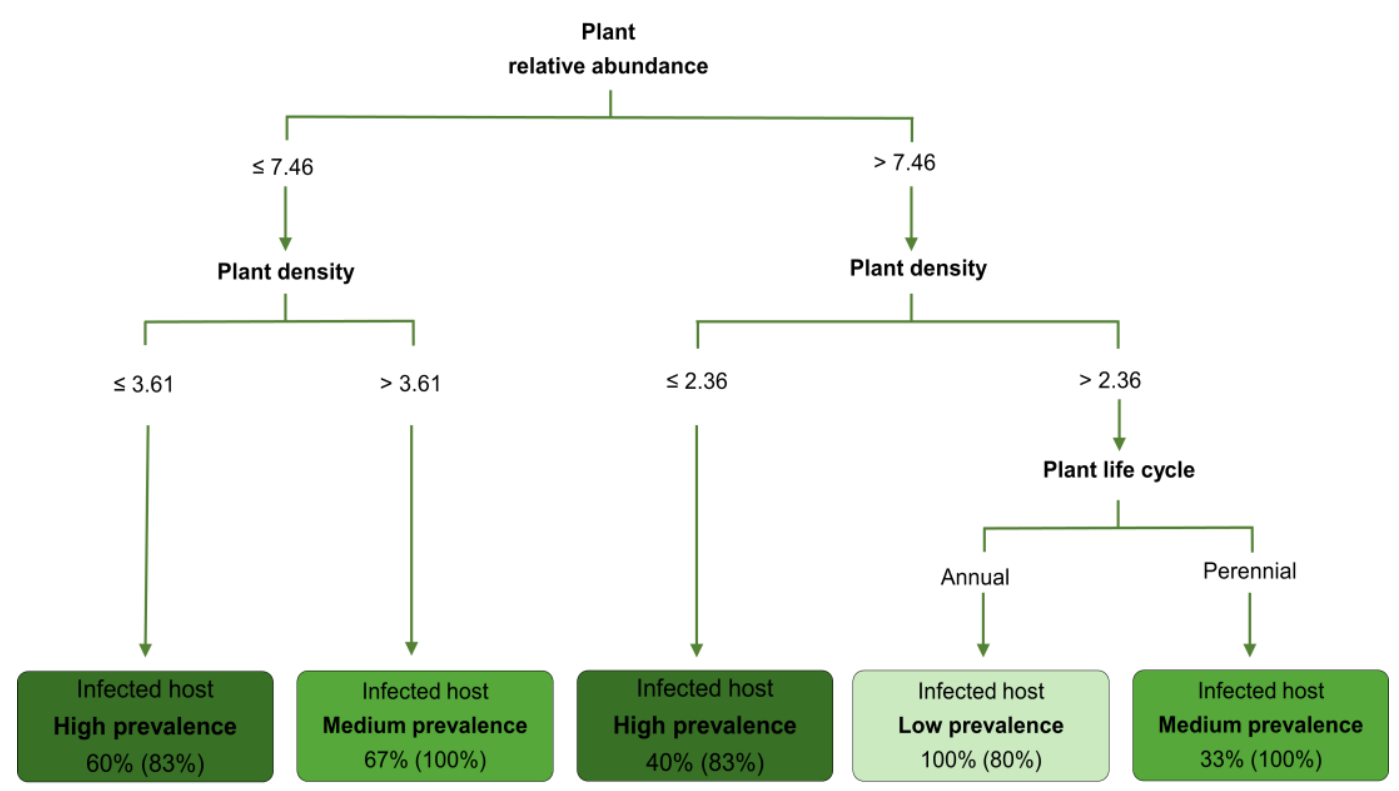

Figure 4.16. Condensed J48 Decision Tree of host potyvirus prevalence in evergreen oak forests. Defining predictors and categories (for categorical predictors) or threshold values (for continuous predictors) of each node are represented. Leaf nodes indicate the predicted prevalence class, the percentage of the total number of instances assigned to that prevalence class (in parenthesis) and the percentage of instances assigned to that prevalence class at the specific leaf node (out of the parenthesis).

(iii) Finally, according to Random Forest methods, in both ecosystems the predictors with the higher relative importance (higher MDI values) were plant density and relative abundance (MDI=0.70-0.85). Plant life cycle was also ranked as the third most important predictor in riparian $(\mathrm{MDI}=0.5)$ and evergreen oak (MDI=0.75) forests. All other predictors presented lower MDI values (Figure 4.17).

These results confirmed that $M L$ models identified plant relative abundance as the most important predictor and showed that plant density and plant life cycle are also important determinants of potyvirus prevalence in the studied ecosystems. Moreover, models that contained these factors improved 
their predictive power when were used to predict level of prevalence on infected hosts plant species. Thus, once potyviruses enter riparian and evergreen oak forests, factors other than plant species identity are the best predictors of the level of virus prevalence.
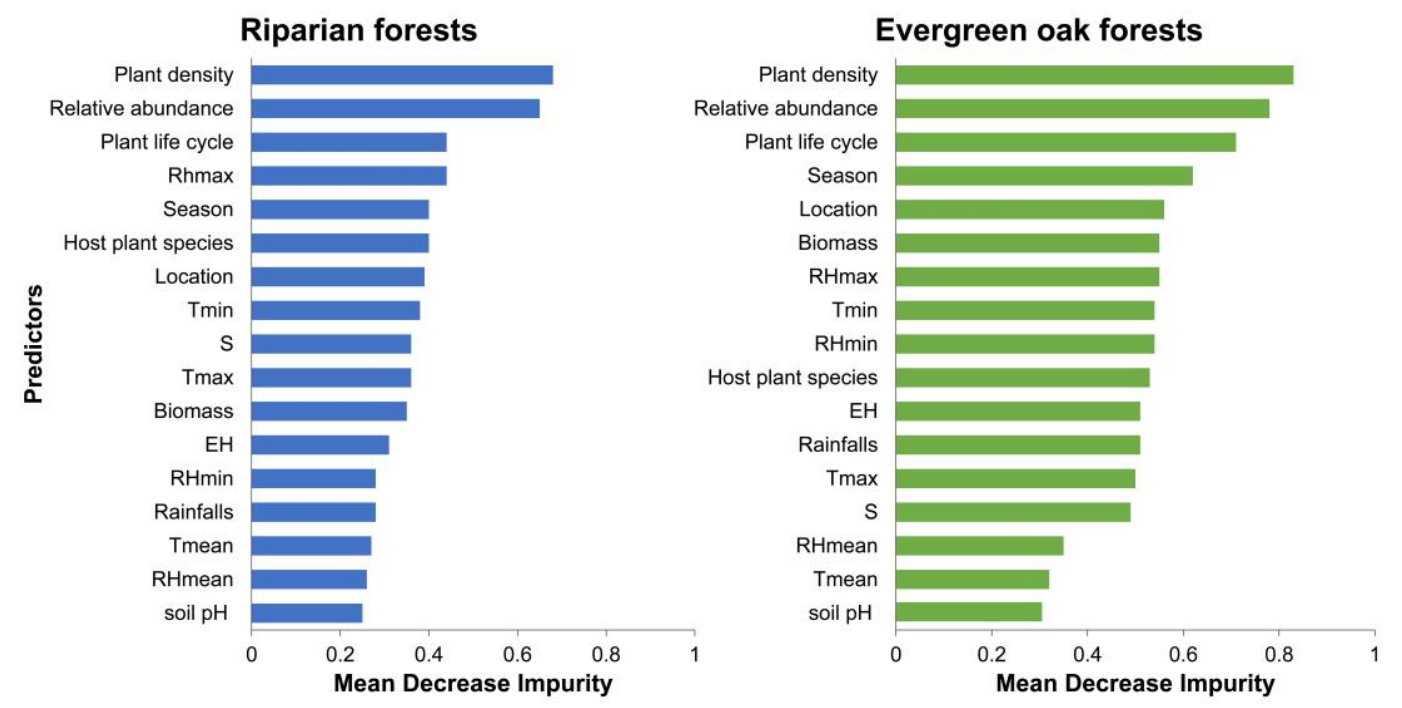

Figure 4.17. Relative importance of the predictors of host potyvirus prevalence according to Mean Decrease Impurity (MDI) values from 100 Random Forest trees. Trees were generated for the riparian forests (blue) and evergreen oak forests (green) datasets, excluding plant identity traits as predictors. Values are normalized in a range from 0 to 1 . The higher the MDI of a predictor, the more relevant the predictor is.

Bivariate analyses indicated that in riparian forests, there was a negative association between the relative abundance of host plant species and potyvirus prevalence $\left(r=-0.25 ; P=1 \times 10^{-5}\right)$ (Figure 4.18A), whereas there was a weak positive correlation between host plant density and potyvirus prevalence $(r=0.10 ; P=0.002)$ (Figure 4.18C). In evergreen oak forests however, the relationship between host plant relative abundance and plant density with potyvirus prevalence followed the same trend than in riparian forests, but the association between these traits was not significant $(r<0.09$; $P>0.103$ ) (Figures 4.18B and D). 


\section{Riparian forests}

A.

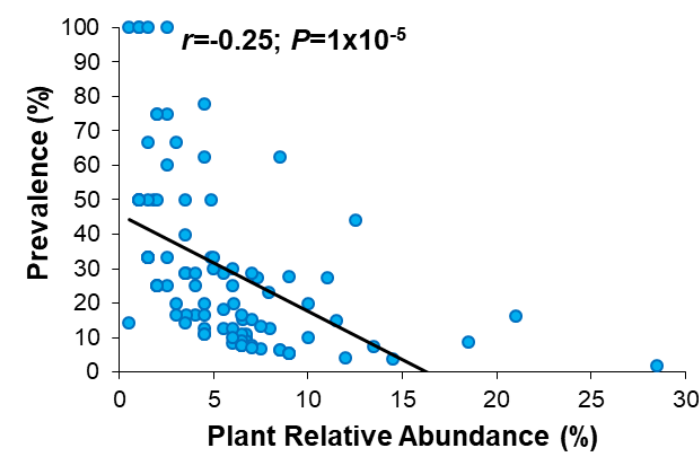

C.

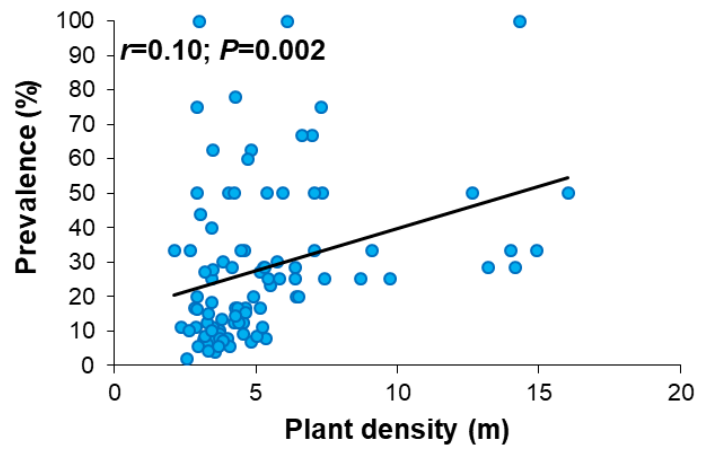

\section{Evergreen oak forests}

B.

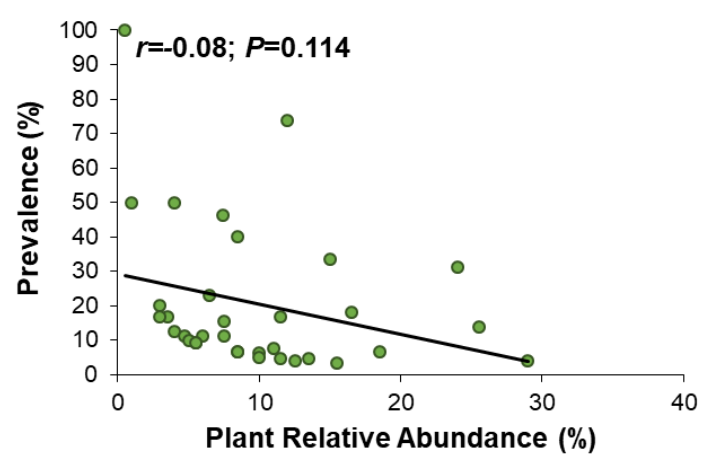

Figure 4.18. Bivariate relationships between host potyvirus prevalence and the best predictor variables identified in $M L$ models. Regressions of plant relative abundance $(A)$ and $(B)$, and plant density (C) and (D) on potyvirus prevalence in riparian forests (blue) and evergreen oak forests (green) are represented. 


\subsection{Analysis of species-specific potyvirus prevalence in wild ecosystems in the Iberian Peninsula}

In section 4.2, ecological factors affecting genus-wide potyvirus prevalence in evergreen oak and riparian forests were analysed. Although these analyses yield relevant information to understand virus epidemiology in the monitored ecosystems, for certain virus species some ecological factor may be more important than others, according to the specific characteristics of such virus species. Thus, analyses at the genus-wide level may mask the differential importance of a given ecological factor at the species-specific level (Jones, 2009). It has been proposed that one of the traits that may influence the relative importance of ecological factors in virus prevalence is the virus host range (Malpica et al., 2006, Pagán et al., 2012; McLeish et al., 2017). For instance, for a generalist virus (i.e., that with a wide host range), host density might be the key ecological factor: as a significant number of plant species would be hosts, the relevant factor for virus prevalence is how close are plants to each other. On the other hand, for a specialist virus (i.e., that with a narrow host range), plant species richness might be the relevant factor: as few species are virus hosts, the number of plant species would determine the chances for the virus to find a susceptible plant (Pagán et al., 2012; García-Arenal and Fraile, 2013; Johnson et al., 2015). Following this rationale, in the present chapter the importance of ecological factors in determining potyvirus species-specific prevalence is analysed. The analyses focus in two of the potyvirus species detected in evergreen oak and riparian forests: one that follows a specialist strategy and another that follows a generalist strategy.

\subsubsection{Identification of potyvirus species}

Potyvirus species present in evergreen oak and riparian forests were identified by sequencing the PCR products obtained with the universal primers described by Zhang et al., 2010, which correspond to a 350nt fragment of the NIb (see section 3.2.2 of Materials and Methods). A total of $87 \mathrm{Nlb}$ fragments from the same number of potyvirus isolates were obtained. Using this sequence data set, analyses of nucleotide sequence identity between the amplified fragments and 
the same region of the genome from potyvirus sequences available in GenBank (Table 4.21), and Maximum Likelihood-based inference of phylogenetic relationships between these sequences, were performed (Figure 4.19). Virus species identification was confirmed by sequencing the CP gene of the same isolates for which the 350nt NIb fragment was obtained, and following the same procedure (Table 4.22 and Figure 4.20).

Table 4.21. Nucleotide sequence identity of the four identified virus species with the phylogenetically closest potyvirus species based on the 350nt Nlb fragment.

\begin{tabular}{lccc}
\hline Identified potyvirus species & $\mathbf{N}$ & Phylogenetically closest potyvirus & $\begin{array}{c}\text { Sequence } \\
\text { identity (\%) }\end{array}$ \\
\hline $\begin{array}{c}\text { Clover yellow vein virus } \\
\text { Endive necrotic mosaic virus }\end{array}$ & 14 & $\begin{array}{c}\text { Clover yellow vein virus } \\
\text { Endive necrotic mosaic virus }\end{array}$ & $95-97$ \\
Mediterranean ruda virus & 40 & Bean yellow mosaic virus & 79 \\
& & Clover yellow vein virus & $74-76$ \\
Wild hop mosaic virus & 31 & Papaya ringspot virus & $54-56$ \\
N: number of sequences. \\
* Range of nucleotide identity between the sequenced isolates and representative sequences of the \\
phylogenetically closest potyvirus species.
\end{tabular}

Both nucleotide Nlb-based nucleotide identity and phylogenetic analyses indicated the presence of four potyvirus species:

(i) 14 sequences had $95-97 \%$ nucleotide identity (Table 4.21), and clustered in the same monophyletic group in the phylogenetic tree (Figure 4.19), with the available sequences of Clover yellow vein virus (CIYVV) and were therefore categorized as belonging to CIYVV. Similar identity $(94-97 \%$, Table 4.22) and phylogenetic position (Figure 4.20) were obtained in the analysis of $\mathrm{CP}$ gene.

(ii) Two sequences shared $79 \%$ nucleotide identity with the unique Nlb sequence of Endive necrotic mosaic virus (ENMV) available in GenBank (Table 4.21) and clustered together with this reference sequence (Figure 4.19), and were identified as belonging to ENMV. This result could not be confirmed, as the CP gene of these two virus isolates could not be obtained. 


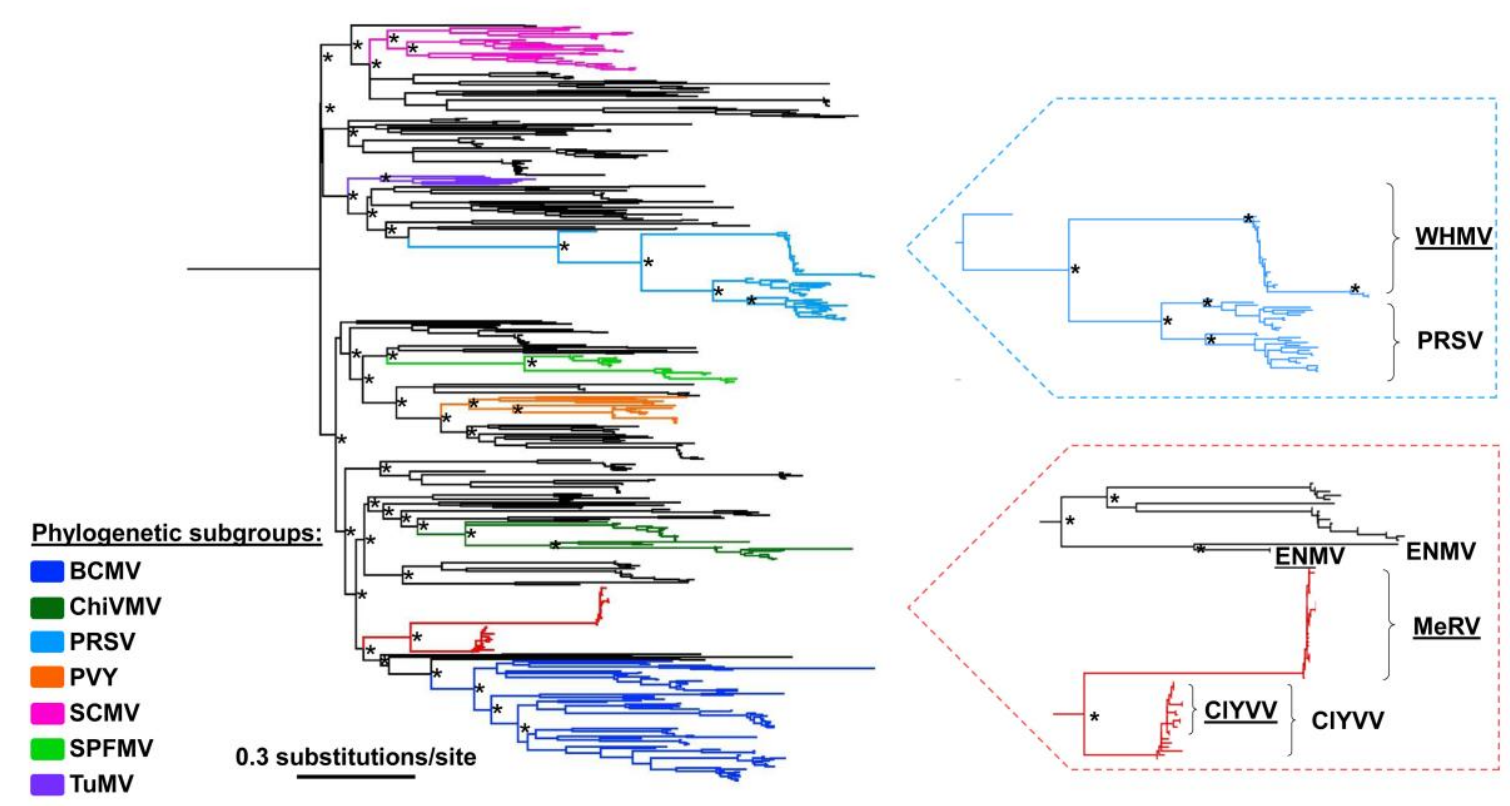

Figure 4.19. Maximum likelihood phylogenetic tree based on the nucleotide sequence of a $350 \mathrm{nt}$ fragment of the $\mathrm{Nlb}$ gene. Asterisks indicate nodes with $\mathrm{SH}$-like support of $\geq 0.80$. The tree is mid-point rooted. Phylogenetic subgroups of potyviruses defined by Shukla et al., 1994 were used as reference and indicated in coloured branches. BCMV: Bean common mosaic virus; ChiVMV: Chilli veinal mottle virus; PRSV: Papaya ringspot virus; PVY: Potato virus Y; SCMV: Sugarcane mosaic virus; SPFMV: Sweet potato feathery mottle virus; TuMV: Turnip mosaic virus. Expansions of the phylogeny contain the group of virus sequences in which potyvirus isolates sequenced in this study (underlined) clustered. CIYVV: Clover yellow vein virus; ENMV: Endive necrotic mosaic virus; MeRV: Mediterranean ruda virus; WHMV: Wild hop mosaic virus.

(iii) 31 partial Nlb sequences had $73-78 \%$ nucleotide identity with Papaya ringspot virus (PRSV) (Table 4.21) and clustered in a monophyletic group closely related, but separated, to the existing PRSV Nlb sequences (Figure 4.19). As most isolates had nucleotide identities below $76 \%$, they were considered as belonging to a non-previously described species of the genus Potyvirus (Adams et al., 2005). Analyses of the CP gene of these isolates indicated a nucleotide identity of $71-73 \%$ with the reference sequence of Zucchini yellow fleck virus (ZYFV) (Table 4.22), for which no Nlb sequence was available. ZYFV was the phylogenetically closest potyvirus (Figure 4.20), PRSV being the second closest. As the species demarcation criteria for potyviruses based on CP sequence identity is lower than $76 \%$ (King et al., 2012), this result confirmed that this group of 31 sequences belonged to a no-described virus 
species provisionally named as Wild hop mosaic virus (WHMV). The name was chosen because this species was detected with the highest prevalence in hop plants (Humululs lupulus) of wild ecosystems (see potyvirus host range in section 4.3.2).

(iv) The remaining 40 partial Nlb sequences showed $74-76 \%$ nucleotide identity with sequences of Bean yellow mosaic virus (BYMV) isolates available in GenBank (Table 4.21). However, in the Nlb-based phylogenetic analysis these 40 sequences formed a cluster closely related to CIYVV sequences (Figure 4.19) with which they shared 54-56\% nucleotide identity (Table 4.21). The position of these 40 partial Nlb sequences in the tree with respect to BYMV could not be determined because nodes containing BYMV sequences were not statistical supported. However, the analysis of the CP sequences was more consistent: it showed a nucleotide identity of $70 \%$ between the 40 sequenced CPs and the BYMV reference CP sequences (Table 4.22). Also, both groups of sequences grouped in different, but closely related, monophyletic groups (Figure 4.20). According to ICTV rules, these results indicated that the 40 sequences were from a novel species of the genus Potyvirus that was provisionally named Mediterranean ruda virus (MeRV) because this species was detected exclusively in mountain rue plants (Ruta montana) (see potyvirus host range in section 4.3.2).

Table 4.22. Nucleotide sequence identity of three identified virus species with the phylogenetically closest potyvirus species based on the CP.

\begin{tabular}{cccc}
\hline Identified potyvirus species & $\mathbf{N}$ & Phylogenetically closest potyvirus & $\begin{array}{c}\text { Sequence } \\
\text { identity }(\%)^{\star}\end{array}$ \\
\hline Clover yellow vein virus & 12 & Clover yellow vein virus & $94-97$ \\
Mediterranean ruda virus & 40 & Bean yellow mosaic virus & 70 \\
& & Clover yellow vein virus & $66-67$ \\
Wild hop mosaic virus & \multirow{2}{*}{4} & Zucchini yellow fleck virus & $71-73$ \\
& & Papaya ringspot virus & 70 \\
\hline
\end{tabular}

$\mathrm{N}$ : number of sequences.

${ }^{*}$ Range of nucleotide identity between the sequenced isolates and representative sequences of the phylogenetically closest potyvirus species. 


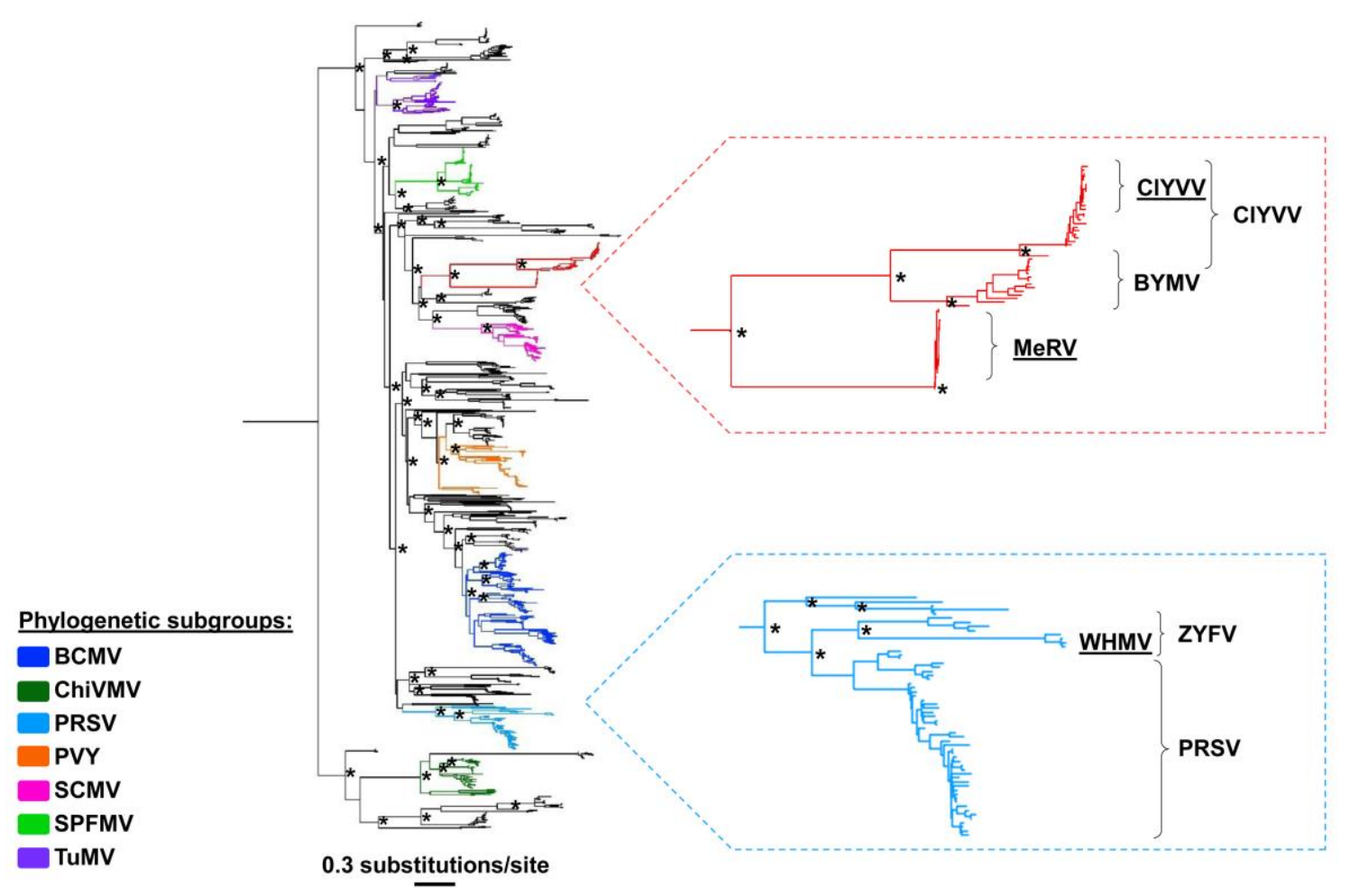

Figure 4.20. Maximum likelihood phylogenetic tree based on the nucleotide sequence of the CP gene. Asterisks indicate nodes with $\mathrm{SH}$-like support of $\geq 0.80$. The tree is mid-point rooted. Phylogenetic subgroups of potyviruses defined by Shukla et al., 1994 were used as reference and indicated in coloured branches. BCMV: Bean common mosaic virus; ChiVMV: Chilli veinal mottle virus; PRSV: Papaya ringspot virus; PVY: Potato virus Y; SCMV: Sugarcane mosaic virus; SPFMV: Sweet potato feathery mottle virus; TuMV: Turnip mosaic virus. Expansion of the phylogeny section contains the group of virus sequences in which potyvirus isolates of this study (underlined) are clustered. CIYVV: Clover yellow vein virus; BYMV: Bean yellow mosaic virus; MeRV: Mediterranean ruda virus; ZYFV: Zucchini yellow fleck virus; WHMV: Wild hop mosaic virus.

\subsubsection{Potyvirus species-specific host range and prevalence}

The host range of the identified potyvirus species was determined. Two virus species (ENMV and MeRV) were found in a single host species, whereas the other two (CIYVV and WHMV) were detected infecting plant species from different botanical families (Table 4.23). The two viruses with broader host ranges had a broader geographical distribution than virus species with a narrower host range (Table 4.23). 
Table 4.23. Host range and geographical distribution of potyvirus species identified in the studied ecosystems.

\begin{tabular}{|c|c|c|c|}
\hline $\begin{array}{l}\text { Potyvirus } \\
\text { species }\end{array}$ & Ecosystem & Location & Host species \\
\hline \multirow[t]{4}{*}{ CIYVV } & \multirow[t]{4}{*}{ Riparian forest } & El Escorial & $\begin{array}{c}\text { Trifolium campestre (Fabaceae) } \\
\text { Trifolium pratense(Fabaceae) }\end{array}$ \\
\hline & & \multirow[t]{3}{*}{ Palazuelos } & Rumex pulcher (Polygonaceae) \\
\hline & & & Trifolium campestre (Fabaceae) \\
\hline & & & Trifolium pratense (Fabaceae) \\
\hline ENMV & Riparian forest & $\begin{array}{l}\text { Santa Cruz de } \\
\text { Retamar }\end{array}$ & Andryala ragusina (Asteraceae) \\
\hline MeRV & Evergreen oak forest & El Pardo & Ruta montana (Rutaceae) \\
\hline \multirow[t]{8}{*}{ WHMV } & Evergreen oak forest & Marjaliza & Cistus albidus (Cistaceae) \\
\hline & \multirow[t]{7}{*}{ Riparian forest } & El Escorial & Trifolium pratense (Fabaceae) \\
\hline & & Palazuelos & Trifolium campestre (Fabaceae) \\
\hline & & & Trifolium pratense (Fabaceae) \\
\hline & & Pollos & Ecballium elaterium (Cucurbitaceae) \\
\hline & & & Humulus lupulus (Cannabaceae) \\
\hline & & \multirow{2}{*}{$\begin{array}{l}\text { Santa Cruz de } \\
\text { Retamar }\end{array}$} & Humulus lupulus (Cannabaceae) \\
\hline & & & Salix atrocinerea (Salicaceae) \\
\hline
\end{tabular}

CIYVV: Clover yellow vein virus; ENMV: Endive necrotic mosaic virus; MeRV: Mediterranean ruda virus; WHMV: Wild hop mosaic virus

CIYVV was detected infecting three plant species in two locations of riparian forest (Table 4.23): In El Escorial, CIYVV infected the perennial plant Trifolium pratense and the annual plant Trifolium campestre, and in Palazuelos the virus infected these two hosts plus the perennial plant Rumex pulcher. The prevalence of CIYVV ranged between $4.17 \%$ and $28.57 \%$ in these two locations (Appendix 4, Table A4.1). ENMV was found occasionally infecting individuals of the perennial plant Andryala ragusina in the riparian forest of Santa Cruz de Retamar, with prevalence between $10.00 \%$ and $11.50 \%$ (Appendix 4, Table A4.2). The remaining two viruses (MeRV and WHMV) accounted for the largest fraction of infected plant species. MeRV was detected in the perennial plant Ruta montana in the evergreen oak forests of El Pardo (Table 4.23), and showed a prevalence of $18.18-73.91 \%$ (see Table 4.24). WHMV infected mostly perennial plant species collected in riparian forests (Table 4.23): Trifolium pratense in El Escorial and Palazuelos; Humulus lupulus in Pollos and Santa Cruz de Retamar; Ecballium elaterium in Pollos; and Salix atrocinerea in Santa Cruz de Retamar. WHMV was also detected in the annual plant Trifolium 
campestre at Palazuelos (Table 4.23). In these hosts, prevalence of WHMV ranged between $5.41 \%$ and $100 \%$ during the monitored period (see Table 4.27). Exceptionally (one single plant), the virus was found in the evergreen oak forest of Marjaliza infecting the perennial plant Cistus albidus. Overall, potyvirus species identified in this thesis mostly infected perennial plants, with host range and prevalence varying according to virus species, geographical location and season.

Thus, the identified virus species could be classified into two groups according to their host range: MeRV and EMNV behaved as specialists, whereas WHMV and CIYVV behaved as generalists. To further study how the ecological factors recorded in evergreen oak and riparian forests could differentially influence virus prevalence according to the virus host range, further analyses focused in the most prevalent representative species of each strategy: MeRV as a specialist virus, and WHMV as a generalist virus.

\subsubsection{Ecological factors affecting MeRV prevalence}

\subsubsection{Analysis of MeRV prevalence}

As mentioned in the previous section, MeRV behaved as a specialist virus, as its host range was limited to the perennial plant mountain rue that was present only in evergreen oak forests. Indeed, the virus was highly prevalent in this host plant. In order to perform a more accurate analysis of the ecological determinants of MeRV prevalence, additional samplings were performed in locations of evergreen oak forests during a third sampling year (summer and autumn 2015 and spring 2016). Infection by MeRV was detected in every season during this third sampling year, as it was in the previous two years (Table 4.24). In total, MeRV prevalence was analysed in $98 \%$ (301/306) of the mountain rue plants collected in evergreen oak forests between summer 2013 and spring 2016. The virus was detected in 123/301 (41\%). 
Table 4.24. Season-specific average values of MeRV prevalence and of ecological/climatic factors measured in evergreen oak forest location.

\begin{tabular}{|c|c|c|c|c|c|c|c|c|c|}
\hline \multirow[b]{2}{*}{ Parameter } & \multicolumn{3}{|c|}{ Sampling cycle 1} & \multicolumn{3}{|c|}{ Sampling cycle 2} & \multicolumn{3}{|c|}{ Sampling cycle 3} \\
\hline & $\begin{array}{c}\text { Summer } \\
2013\end{array}$ & $\begin{array}{c}\text { Autumn } \\
2013\end{array}$ & $\begin{array}{l}\text { Spring } \\
2014\end{array}$ & $\begin{array}{c}\text { Summer } \\
2014\end{array}$ & $\begin{array}{c}\text { Autumn } \\
2014\end{array}$ & $\begin{array}{c}\text { Spring } \\
2015\end{array}$ & $\begin{array}{c}\text { Summer } \\
2015\end{array}$ & $\begin{array}{c}\text { Autumn } \\
2015\end{array}$ & $\begin{array}{l}\text { Spring } \\
2016\end{array}$ \\
\hline $\mathbf{N}$ & 13 & 30 & 15 & 23 & 48 & 33 & 68 & 38 & 33 \\
\hline Prevalence & 46.15 & 33.33 & 40.00 & 73.91 & 31.25 & 18.18 & 32.35 & 71.05 & 45.45 \\
\hline $\begin{array}{c}\text { Relative } \\
\text { abundance }\end{array}$ & 0.07 & 0.15 & 0.08 & 0.12 & 0.24 & 0.16 & 0.34 & 0.19 & 0.16 \\
\hline $\begin{array}{l}\text { Host plant } \\
\text { density }\end{array}$ & 2.94 & 2.36 & 2.36 & 2.54 & 2.43 & 2.32 & 2.39 & 2.41 & 2.46 \\
\hline V & 0.01 & 0.05 & 0.05 & 0.01 & 0.04 & 0.08 & 0.00 & 0.10 & 0.02 \\
\hline$S$ & 24 & 15 & 26 & 25 & 18 & 27 & 9 & 18 & 19 \\
\hline$T_{\max }$ & 34.47 & 24.90 & 28.37 & 36.30 & 29.10 & 25.85 & 40.70 & 22.90 & 31.45 \\
\hline $\mathbf{T}_{\min }$ & 8.80 & -1.45 & 2.87 & 11.33 & 5.93 & 0.70 & 18.20 & -2.20 & 14.51 \\
\hline $\mathbf{T}_{\text {mean }}$ & 22.40 & 12.70 & 14.50 & 23.70 & 16.90 & 12.50 & 29.00 & 11.40 & 23.00 \\
\hline $\mathbf{R H}_{\max }$ & 85.67 & 90.00 & 89.00 & 86.33 & 89.33 & 88.50 & 83.00 & 89.00 & 89.00 \\
\hline $\mathbf{R H}_{\min }$ & 17.67 & 23.00 & 18.67 & 17.67 & 26.33 & 18.00 & 14.00 & 21.00 & 19.00 \\
\hline $\mathbf{R H}_{\text {mean }}$ & 39.00 & 53.00 & 46.00 & 37.00 & 57.00 & 49.00 & 49.00 & 55.00 & 48.00 \\
\hline Rainfalls & 95.00 & 216.00 & 230.00 & 42.00 & 419.00 & 348.00 & 94.00 & 136.00 & 289.00 \\
\hline
\end{tabular}

$\mathrm{N}$ : Total number of analysed plants per population.

Prevalence: percentage of infected plants of mountain rue (Ruta montana).

$\mathrm{V}$ : Plant biomass $\left(\mathrm{m}^{3}\right)$.

S: Species richness.

$T_{\max }, T_{\min }, T_{\text {mean }}$ : Average of monthly maximum, minimum and mean temperatures $\left({ }^{\circ} \mathrm{C}\right)$ in a season.

$\mathrm{RH}_{\max }, \mathrm{RH}_{\text {min }}, \mathrm{RH}_{\text {mean }}$ : Average of monthly maximum, minimum and mean relative humidity (\%) in a season.

Rainfalls: Average of total rainfalls $(\mathrm{mm})$ in a season.

MeRV prevalence in mountain rue plants varied across seasons between $18 \%$ in spring 2015 to $74 \%$ in summer 2014 (Table 4.24). Average MeRV prevalence did not vary between sampling cycles $\left(X^{2}<2.21, P>0.137\right)$. However, during the first and second cycle the incidence was higher in summer than in spring and autumn $\left(X^{2}>11.43, P<1 \times 10^{-5}\right)$, whereas in the third sampling cycle the highest incidence occurred in autumn $\left(X^{2}>4.79, P<0.029\right)$ (Table 4.24). Cultivated fields of tomato (Solanum lycopersicum L.) and pepper (Capsicum annuum L.) located near to the sampled locations of evergreen oak forests were also visited to address the possibility that MeRV could also infect crops. Melon (Cucumis melo L.) fields were also included in the surveys, as, together with pepper and tomato, this was the major cultivated plant in the area (Table 4.25). At least two fields from each crop were visited in summer, spring and autumn, and at each visit between 20 and 50 individuals were sampled. For that, three leaves of different branches from one out of every three plants were collected along a fixed itinerary. MeRV was never detected in melon, tomato and pepper plants. 
Table 4.25. Locations of crop fields in the Iberian Peninsula visited between 2013 and 2016.

\begin{tabular}{lcccc}
\hline Location & Ecosystem & Latitude & Longitude & Elevation $(\mathbf{m})$ \\
\hline $\begin{array}{l}\text { Cortijo de San Isidro } \\
\text { Villamanrique de Tajo }\end{array}$ & $\begin{array}{l}\text { Cultivated/Melon } \\
\text { Cultivated/Melon }\end{array}$ & 40.054 & -3.580 & 494 \\
& & 40.083 & -3.216 & 559 \\
Cortijo de San Isidro & Cultivated/Tomato & 40.056 & -3.579 & 496 \\
Ciruelos de Pradales & Cultivated/Tomato & 41.439 & -3.705 & 1161 \\
& & & & \\
Cortijo de San Isidro & Cultivated/Pepper & 40.055 & -3.579 & 495 \\
Ciruelos de Pradales & Cultivated/Pepper & 41.439 & -3.703 & 1164 \\
\hline
\end{tabular}

\subsubsection{Analysis of ecological factors affecting MeRV prevalence}

To further understand the ecological determinants of MeRV prevalence in evergreen oak forest, the following ecological factors were considered as predictors: host plant density and relative abundance, species richness, plant biomass, temperature and relative humidity (minimal, maximal and average values), and rainfall in the sampled locations (Table 4.24). The lower number of observations in this data set, as compared with that used in section 4.2, prevented analysing the association between the ecological factors and MeRV prevalence using $\mathrm{ML}$ methods. Instead, multiple regression model selection analyses were used. In these analyses, the continuous values of the dependent variable were used as data followed a normal distribution (using the ShapiroWilk normality test, $W=0.90, P>0.267$ ) (at odds with data used in section 4.2), and season was included as a covariate to account for the dependency of MeRV prevalence over time (Table 4.24). The best-ranked model contained plant density as the only predictor, this model closely competed with that containing rainfall as the only predictor (Table 4.26). The other models showed poorer predictive power $(\Delta>2)$.

Table 4.26. Model selection analyses for MeRV prevalence. Best-ranked models are shown.

\begin{tabular}{|c|c|c|c|c|c|}
\hline Model structure & $r^{\dagger}$ & logLik & AIC $\ddagger$ & $\Delta_{\mathrm{i}}^{\S}$ & $\omega_{i}^{i}$ \\
\hline HPD (100) & $0.68^{*}$ & -22.44 & 52.87 & 0.00 & 0.52 \\
\hline RAF (100) & $-0.68^{*}$ & -22.51 & 53.03 & 0.16 & 0.48 \\
\hline
\end{tabular}

"The relative importance (\%) of each predictor variable is shown in parenthesis.

${ }^{\dagger}$ Correlation coefficient. Asterisks indicate significant correlations $(P<0.05)$.

¥Akaike's Information Criterion.

${ }_{\Delta} \Delta$, is the difference between the AIC of a given model and that of the best-ranked model, and quantifies how models compete (best-ranked model: $\Delta_{i}=0$; substantial empirical support: $\Delta_{i}=1-2$; considerable less support: $\Delta_{i}=2-7$; and no support; $\Delta>10$ ) (Burnham and Anderson, 2002).

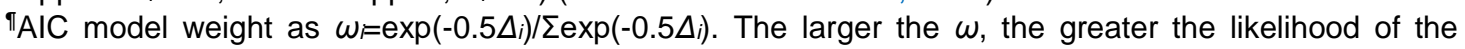
model relatively to the competing models. Maximum $\omega,=1$.

HPD: Host plant density; RAF: Rainfalls. 
Bivariate analyses indicated that there was a positive association between mountain rue density and MeRV prevalence ( $r=0.68 ; P=0.046)$ (Figure 4.21A), whereas rainfall was negatively associated with MeRV prevalence ( $r=-0.68 ; P=0.052$ ) (Figure 4.21B).
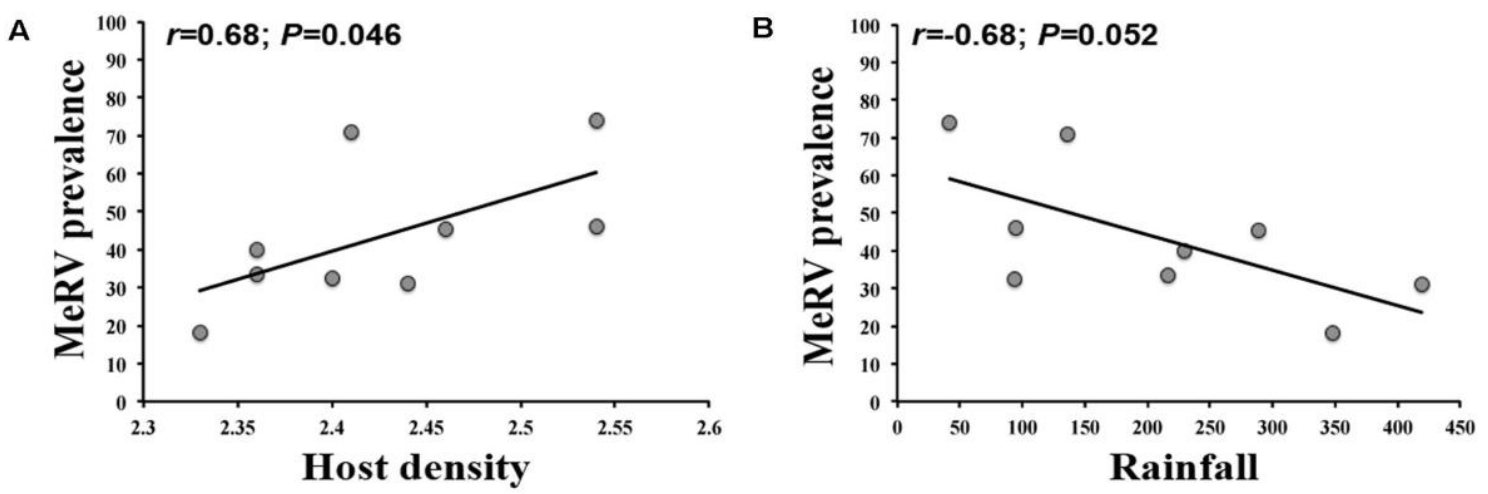

Figure 4.21. Bivariate relationships between MeRV prevalence and the best predictor variables identified by model selection analyses. Regressions of host density (A) and rainfall (B) on MeRV prevalence are represented.

\subsubsection{Ecological factors affecting WHMV prevalence}

\subsubsection{Analysis of WHMV prevalence}

WHMV infected six different host plant species from taxonomically distant plant families in four riparian forest locations (Table 4.27). Additional samples of WHMV hosts were also collected in locations where the virus was present for a third sampling cycle in order to increase the time span of the study (Table 4.27). Infection by WHMV was detected in every season during the monitored period and prevalence was calculated as the percentage of infected plants relative to the total number of plants collected from a given host species. WHMV presence was analysed in 454 host plant samples (6 to 141 samples, depending on the host species). At odds with MeRV, WHMV was not detected in every season and at every location during the monitored period. WHMV prevalence varied across seasons between $8.33 \%$ in summer 2015 to $100 \%$ in spring 2014 and summer 2015. Overall, higher average prevalence was recorded in summer and spring $\left(X^{2}>9.70, P<0.002\right)$ than in autumn $\left(X^{2}<3.46, P>0.063\right)$. Average WHMV prevalence varied significantly between the first and the second cycles $\left(X^{2}=5.40, P=0.020\right)$, but there were not significant differences between these cycles and third sampling cycle $\left(X^{2}<3.23, P>0.072\right)$ (Table 4.27). 
Table 4.27. Prevalence of WHMV in riparian forests during the monitored period.

\begin{tabular}{|c|c|c|c|c|c|c|c|c|c|c|c|c|c|c|}
\hline \multirow[b]{3}{*}{ Season } & \multirow{2}{*}{\multicolumn{2}{|c|}{$\begin{array}{c}\text { EL ESCORIAL } \\
\begin{array}{c}\text { Trifolium } \\
\text { pratense }\end{array}\end{array}$}} & \multicolumn{4}{|c|}{ PALAZUELOS } & \multicolumn{4}{|c|}{ POLLOS } & \multicolumn{4}{|c|}{ SANTA CRUZ DE RETAMAR } \\
\hline & & & \multirow[b]{2}{*}{$\mathbf{N}$} & \multirow{2}{*}{$\begin{array}{l}\begin{array}{l}\text { Trifolium } \\
\text { pratense }\end{array} \\
\text { WHMV (\%) }\end{array}$} & \multicolumn{2}{|c|}{$\begin{array}{l}\text { Trifolium } \\
\text { campestre }\end{array}$} & \multicolumn{2}{|c|}{$\begin{array}{l}\text { Humulus } \\
\text { lupulus }\end{array}$} & \multicolumn{2}{|c|}{$\begin{array}{l}\text { Ecballium } \\
\text { elaterium }\end{array}$} & \multicolumn{2}{|c|}{$\begin{array}{c}\text { Humulus } \\
\text { lupulus }\end{array}$} & \multicolumn{2}{|c|}{$\begin{array}{c}\text { Salix } \\
\text { atrocinerea }\end{array}$} \\
\hline & $\mathbf{N}$ & WHMV (\%) ${ }^{1}$ & & & $\mathbf{N}$ & WHMV (\%) ${ }^{1}$ & $\mathbf{N}$ & WHMV (\%) ${ }^{1}$ & $\mathbf{N}$ & WHMV (\%) ${ }^{1}$ & $\mathbf{N}$ & WHMV (\%) ${ }^{1}$ & $\mathbf{N}$ & WHMV (\%) ${ }^{1}$ \\
\hline \multicolumn{15}{|c|}{ Sampling cycle 1} \\
\hline Summer 2013 & 10 & 0.00 & 15 & 0.00 & 8 & 0.00 & 6 & 66.67 & 0 & - & 1 & 0.00 & 2 & 50.00 \\
\hline Autumn 2013 & 17 & 0.00 & 18 & 0.00 & 7 & 0.00 & 0 & - & 0 & - & 0 & - & 1 & 0.00 \\
\hline Spring 2014 & 13 & 0.00 & 21 & 0.00 & 6 & 0.00 & 1 & 0.00 & 2 & 100.00 & 4 & 50.00 & 2 & 0.00 \\
\hline \multicolumn{15}{|c|}{ Sampling cycle 2} \\
\hline Summer 2014 & 12 & 0.00 & 20 & 0.00 & 0 & - & 4 & 25.00 & 3 & - & 1 & 0.00 & 3 & 0.00 \\
\hline Autumn 2014 & 18 & 11.11 & 24 & 0.00 & 20 & 0.00 & 14 & 0.00 & 0 & - & 0 & - & 2 & 0.00 \\
\hline Spring 2015 & 22 & 22.73 & 37 & 5.41 & 14 & 21.43 & 11 & 63.64 & 0 & - & 2 & 50.00 & 2 & 0.00 \\
\hline \multicolumn{15}{|l|}{ Sampling cycle 3} \\
\hline Summer 2015 & 12 & 8.33 & 4 & 0.00 & 1 & 0.00 & 1 & 0.00 & 1 & 100.00 & 0 & - & 0 & - \\
\hline Autumn 2015 & 18 & 0.00 & 4 & 0.00 & 0 & - & 4 & 0.00 & 0 & - & 14 & 7.14 & 2 & 0.00 \\
\hline Spring 2016 & 19 & 15.79 & 8 & 0.00 & 0 & - & 19 & 10.53 & 0 & - & 4 & 25.00 & 0 & - \\
\hline
\end{tabular}

$\mathrm{N}$ : Total number of analysed plants per population.

1 Prevalence calculated as percentage of WHMV-infected plants relative to the total number of plants analysed from a given host species. 
RESULTS

Table 4.28. Average value of the ecological and climatic factors used as predictors of WHMV prevalence.

\begin{tabular}{|c|c|c|c|c|c|c|c|c|c|c|c|c|}
\hline Season & Location $^{1}$ & Relative abundance (\%) & Plant density & Biomass $\left(\mathrm{m}^{3}\right)$ & $\mathbf{S}$ & $T_{\max }$ & $T_{\min }$ & $T_{\text {mean }}$ & $\mathbf{R H}_{\max }$ & $\mathbf{R H}_{\min }$ & $\mathbf{R H _ { \text { mean } }}$ & Rainfalls \\
\hline \multicolumn{13}{|l|}{ Sampling cycle 1} \\
\hline Summer 2013 & ESC & 5.50 & 2.98 & 0.38 & 29.00 & 32.95 & 7.55 & 20.43 & 88.75 & 13.50 & 39.50 & 159.00 \\
\hline Autumn 2013 & ESC & 8.50 & 3.29 & 0.10 & 28.00 & 27.07 & 2.43 & 14.77 & 99.67 & 19.00 & 59.00 & 434.67 \\
\hline Spring 2014 & ESC & 7.00 & 3.46 & 0.13 & 34.00 & 27.07 & 2.47 & 13.43 & 98.67 & 18.67 & 50.00 & 431.00 \\
\hline Summer 2013 & PAL & 18.00 & 5.95 & 0.08 & 31.00 & 31.10 & 5.58 & 18.33 & 94.25 & 14.25 & 46.50 & 216.00 \\
\hline Autumn 2013 & PAL & 40.70 & 6.82 & 0.01 & 32.00 & 25.93 & 0.07 & 13.07 & 98.67 & 17.00 & 63.67 & 277.33 \\
\hline Spring 2014 & PAL & 13.50 & 5.29 & 0.08 & 26.00 & 24.27 & 0.97 & 11.90 & 99.67 & 15.67 & 57.67 & 349.00 \\
\hline Summer 2013 & POL & 3.00 & 6.98 & 0.11 & 32.00 & 32.48 & 6.60 & 18.68 & 96.25 & 14.25 & 49.00 & 222.50 \\
\hline Autumn 2013 & POL & 0.00 & 0.00 & 0.10 & 32.00 & 26.30 & -0.80 & 12.90 & 99.00 & 31.50 & 65.50 & 299.00 \\
\hline Spring 2014 & POL & 1.00 & 13.15 & 0.13 & 35.00 & 25.50 & 1.23 & 12.67 & 99.00 & 18.00 & 56.33 & 166.00 \\
\hline Summer 2013 & SCR & 1.22 & 5.66 & 0.02 & 29.00 & 35.30 & 9.85 & 23.13 & 95.00 & 14.00 & 37.50 & 107.00 \\
\hline Autumn 2013 & SCR & 0.00 & 0.00 & 0.01 & 27.00 & 27.37 & 5.10 & 16.60 & 99.00 & 23.00 & 60.33 & 419.33 \\
\hline Spring 2014 & SCR & 2.00 & 2.91 & 0.06 & 30.00 & 27.97 & 4.50 & 15.30 & 98.00 & 17.00 & 52.67 & 315.33 \\
\hline \multicolumn{13}{|l|}{ Sampling cycle 2} \\
\hline Summer 2014 & ESC & 6.00 & 3.45 & 0.17 & 31.00 & 34.27 & 9.33 & 22.27 & 93.33 & 13.67 & 36.33 & 211.33 \\
\hline Autumn 2014 & ESC & 9.00 & 3.49 & 0.20 & 26.00 & 29.65 & 3.65 & 15.20 & 99.50 & 30.00 & 62.67 & 594.50 \\
\hline Spring 2015 & ESC & 11.00 & 3.18 & 0.15 & 32.00 & 27.53 & 1.27 & 13.70 & 96.00 & 16.33 & 53.00 & 230.67 \\
\hline Summer 2014 & PAL & 20.00 & 3.91 & 0.04 & 23.00 & 32.57 & 6.63 & 20.13 & 96.33 & 12.00 & 42.33 & 138.67 \\
\hline Autumn 2014 & PAL & 34.50 & 3.27 & 0.03 & 30.00 & 27.50 & 4.50 & 14.97 & 98.67 & 24.67 & 63.00 & 507.00 \\
\hline Spring 2015 & PAL & 28.00 & 3.54 & 0.07 & 21.00 & 24.70 & -0.73 & 11.87 & 98.33 & 15.33 & 61.00 & 232.00 \\
\hline Summer 2014 & POL & 2.00 & 5.44 & 0.13 & 35.00 & 34.57 & 7.33 & 20.47 & 93.33 & 12.67 & 44.33 & 80.00 \\
\hline Autumn 2014 & POL & 7.00 & 3.85 & 0.06 & 31.00 & 28.37 & 3.83 & 15.40 & 98.00 & 27.33 & 67.33 & 365.00 \\
\hline Spring 2015 & POL & 5.50 & 3.45 & 0.14 & 32.00 & 27.63 & -0.73 & 12.57 & 97.00 & 14.67 & 60.50 & 101.33 \\
\hline Summer 2014 & SCR & 0.50 & 0.00 & 0.00 & 34.00 & 36.13 & 13.00 & 24.20 & 95.00 & 14.00 & 36.33 & 45.33 \\
\hline Autumn 2014 & SCR & 0.00 & 0.00 & 0.06 & 27.00 & 29.90 & 7.90 & 17.40 & 99.00 & 23.00 & 67.67 & 1031.33 \\
\hline Spring 2015 & SCR & 1.00 & 16.03 & 0.01 & 17.00 & 29.60 & 3.90 & 15.80 & 98.00 & 17.00 & 55.00 & 319.33 \\
\hline \multicolumn{13}{|l|}{ Sampling cycle 3} \\
\hline Summer 2015 & ESC & 6.00 & 2.80 & 0.44 & 25.00 & 39.17 & 13.83 & 26.07 & 88.33 & 12.00 & 37.92 & 124.67 \\
\hline Autumn 2015 & ESC & 9.00 & 2.99 & 0.01 & 26.00 & 26.07 & 3.00 & 15.17 & 98.67 & 20.33 & 60.83 & 364.00 \\
\hline Spring 2016 & ESC & 7.50 & 3.19 & 0.31 & 39.00 & 28.38 & 13.93 & 21.16 & 97.33 & 17.50 & 51.50 & 330.83 \\
\hline Summer 2015 & PAL & 2.50 & 15.50 & 0.00 & 29.00 & 24.70 & -0.73 & 11.87 & 95.67 & 11.67 & - & 320.67 \\
\hline Autumn 2015 & PAL & 1.00 & 14.00 & 0.00 & 29.00 & 24.73 & 2.10 & 13.50 & 99.33 & 17.33 & - & 208.67 \\
\hline Spring 2016 & PAL & 3.50 & 5.17 & 0.03 & 31.00 & 14.16 & 4.48 & 9.32 & - & - & - & 152.00 \\
\hline Summer 2015 & POL & 7.50 & 2.79 & 0.34 & 41.00 & 37.73 & 9.57 & 22.73 & 92.67 & 10.33 & 52.67 & 188.00 \\
\hline Autumn 2015 & POL & 6.00 & 3.23 & 0.04 & 28.00 & 25.37 & 0.33 & 13.23 & 98.00 & 22.00 & 66.42 & 415.33 \\
\hline Spring 2016 & POL & 9.50 & 4.13 & 0.26 & 39.00 & 27.6 & 11.76 & 19.67 & 98.00 & 16.33 & 58.42 & 13367 \\
\hline
\end{tabular}

${ }^{1}$ Locations are designed with a three-letter code: ESC, El Escorial; POL, Pollos; SCR, Santa Cruz de Retamar.

S: Species richness

Tmax, Tmin, Tmean: Average of monthly maximum, minimum and mean temperatures $\left({ }^{\circ} \mathrm{C}\right)$ in a season.

RHmax, RHmin, RHmean: Average of monthly maximum, minimum and mean relative humidity (\%) in a season.

Rainfalls: Average of total rainfalls $(\mathrm{mm})$ in a season.

(-) Missing data. 


\subsubsection{Analysis of ecological factors affecting WHMV prevalence}

To further understand the ecological determinants of WHMV prevalence in riparian forest, the following ecological factors were considered as predictors: host plant density and relative abundance, species richness, plant biomass, temperature and relative humidity (minimal, maximal and average values), and rainfall in the sampled locations. For this analysis, multiple regression model selection analyses were used. However, note than, at odds with MeRV, WHMV was detected in more than one location of riparian forests. Thus, for multiple regression analyses location-specific values in each season and year were used (Table 4.28). The best-ranked model contained plant relative abundance, mean temperature, species richness and plant biomass as predictors $(r=0.79$, $P=0.002$ ), with plant relative abundance having the higher relative importance (63\%) (Table 4.29). The other predictors had much lower relative importance $(<18 \%)$. Ten models closely competed with the best-ranked one $\left(\Delta_{i}<2\right)$. All of these models had similar predictive power than the best-ranked one $(r=0.81$ $0.73, P<0.006$ ), and included plant relative abundance and mean temperature as predictors. Plant relative abundance had always the highest relative importance (46 to $72 \%) \quad(r=0.63, P=0.012) \quad$ (Table 4.29). Some models contained relative humidity and rainfalls as predictors, but always with low relative importance (Table 4.29).

Bivariate analyses indicated that there was a negative association between the relative abundance of host plant species and WHMV prevalence $(r=-0.43 ; P=0.008)$ (Figure 4.22A), whereas plant biomass, mean temperature and species richness were not significantly associated with WHMV prevalence $(r<0.16 ; P>0.118)$ (Figure 4.22B-D).

If the two virus species in which this section is focused are taken as representatives of a specialist and a generalist strategy, the results of this section indicate that the ecological factors determining virus prevalence depend on their host range. The chief predictor of MeRV (specialist) prevalence is the density of host plants, whereas the relative abundance of host plants is the major predictor of WHMV (generalist) prevalence. 
Table 4.29. Model selection analyses for WHMV prevalence. Best-ranked models are shown $\left(\Delta_{i}<2\right)$.

\begin{tabular}{|c|c|c|c|c|c|}
\hline Model structure* & $r^{\dagger}$ & logLik & $\operatorname{AIC}^{\ddagger}$ & $\Delta \Delta_{\mathrm{i}}^{\S}$ & $\omega_{i} i^{\pi}$ \\
\hline PRA (63)+Tmean (18)+S (13)+V (5) & $0.79^{*}$ & -60.67 & 133.35 & 0 & 0.17 \\
\hline PRA (47)+ $T_{\text {mean }}(17)+\mathrm{S}(16)+\mathrm{HPD}(15)+\mathrm{V}(5)$ & $0.81^{*}$ & -60.04 & 134.07 & 0.73 & 0.12 \\
\hline$P R A(51)+T_{\text {mean }}(22)+\mathrm{S}(10)+R H_{\min }(13)+V(5)$ & $0.81^{*}$ & -60.19 & 134.38 & 1.03 & 0.10 \\
\hline $\mathrm{PRA}(63)+\mathrm{T}_{\text {mean }}(18)+\mathrm{S}(16)+\mathrm{RH} \max (3)$ & $0.78^{\star}$ & -61.33 & 134.65 & 1.31 & 0.09 \\
\hline $\mathrm{PRA}(58)+\mathrm{T}_{\text {mean }}(19)+\mathrm{S}(13)+\mathrm{V}(5)+\mathrm{RH} \mathrm{H}_{\text {mean }}(5)$ & $0.80^{*}$ & -60.35 & 134.70 & 1.36 & 0.09 \\
\hline $\mathrm{PRA}(46)+\mathrm{S}(19)+\mathrm{T}_{\text {mean }}(16)+\mathrm{HPD}(16)+\mathrm{RH}_{\max }(3)$ & $0.80^{*}$ & -60.46 & 134.93 & 1.58 & 0.08 \\
\hline $\mathrm{PRA}(60)+\mathrm{T}_{\text {mean }}(18)+\mathrm{S}(14)+\mathrm{V}(5)+\mathrm{RH}_{\max }(3)$ & $0.80^{*}$ & -60.47 & 134.95 & 1.60 & 0.08 \\
\hline$P R A(53)+T_{\text {mean }}(24)+R H_{\min }(17)+V(6)$ & $0.77^{*}$ & -61.52 & 135.04 & 1.69 & 0.07 \\
\hline PRA (57)+ $T_{\text {mean }}(19)+\mathrm{S}(10)+R F A(9)+V(5)$ & $0.80^{*}$ & -60.52 & 135.04 & 1.70 & 0.07 \\
\hline$P R A(72)+T_{\text {mean }}(20)+V(8)$ & $0.73^{*}$ & -62.63 & 135.26 & 1.91 & 0.07 \\
\hline PRA (67)+S (17)+ $T_{\text {mean }}(16)$ & $0.73^{*}$ & -62.67 & 135.33 & 1.99 & 0.06 \\
\hline
\end{tabular}

"The relative importance (\%) of each predictor variable is shown in parenthesis.

${ }^{\dagger}$ Correlation coefficient. Asterisks indicate significant correlations $(P<0.05)$.

¥Akaike’s Information Criterion.

$\S_{\Delta}$, is the difference between the AIC of a given model and that of the best-ranked model, and quantifies how models compete (best-ranked model: $\Delta_{i}=0$; substantial empirical support: $\Delta_{i}=1-2$; considerable less support: $\Delta_{i=2-7}$; and no support; $\Delta>10$ ) (Burnham and Anderson, 2002).

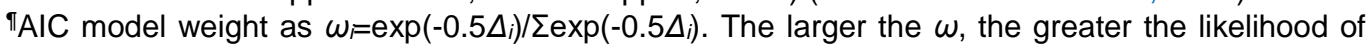
the model relatively to the competing models. Maximum $\omega,=1$.

PRA: Plant relative abundance; Tmean: Average temperature; S: Species richness; HPD: Host plant density; V: Plant biomass; $\mathrm{RH}_{\min }$ : Minimum relative humidity; $\mathrm{RH}_{\max }$ : Maximum relative humidity; RFA: Rainfalls.
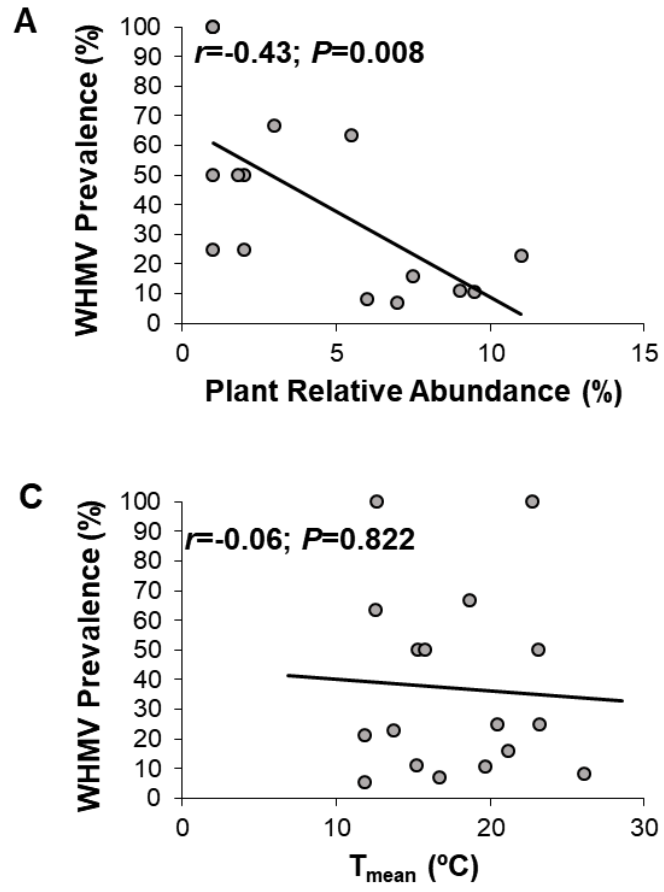
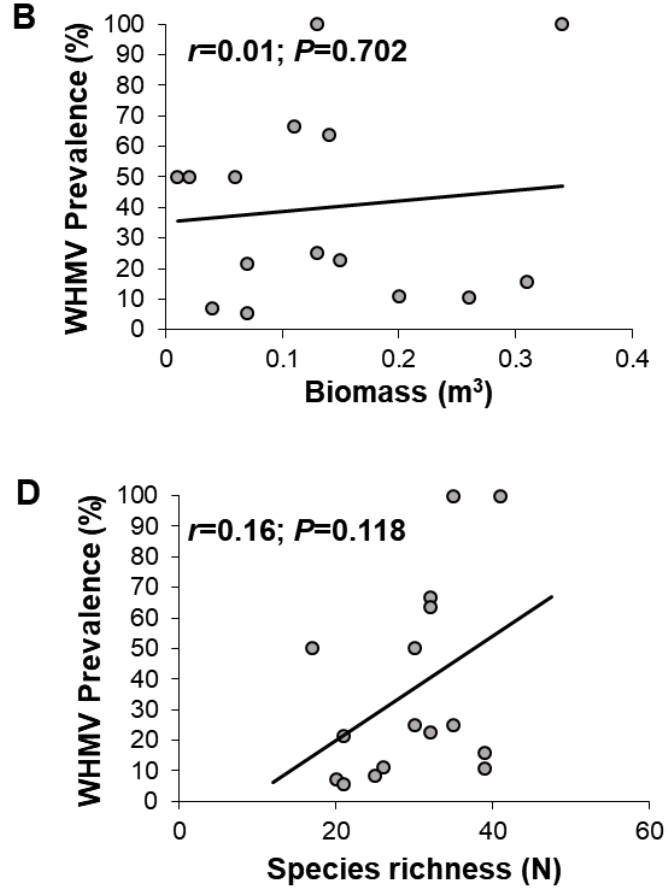

Figure 4.22. Bivariate relationships between WHMV prevalence and the best predictor variables identified by model selection analyses. Regressions of plant relative abundance $(A)$, plant biomass (B), mean temperature (C) and species richness (D) on WHMV prevalence are represented. 


\subsection{Analysis of potyvirus genus-wide genetic diversity in wild ecosystems in the Iberian Peninsula}

In the previous sections, the ecological determinants of potyvirus prevalence were analyzed at the genus and at the species level. These determinants, and their relative importance, varied depending on the taxonomical scale (genus vs. species) of the analysis and on the life strategy (specialist vs. generalist) of the virus species studied. Epidemiological changes may result in genetic modifications in the pathogen population (Grenfell et al., 2004; Archie et al., 2009; Pybus and Rambaut, 2009). Therefore, changes in potyvirus prevalence can be accompanied by variations in the virus population genetic diversity. As for virus epidemiology, evolutionary processes that shape virus population genetic diversity may take place at two levels: (i) macroevolutionary processes, which affect inter-species genetic diversification; and (ii) microevolutionary processes, which shape intra-species genetic diversity. RNA viruses, as are potyviruses, may differ greatly in their modes of macro and microevolution (Kitchen et al., 2011), and ecological factors are thought to shape these processes (Huyse et al., 2005). However, little is known about which and how ecological factors shape virus population genetic diversity at both evolutionary scales (Huyse et al., 2005; Rodelo-Urrego et al., 2015).

To address these questions, in the following sections: (i) the effect of potyvirus prevalence and ecosystem ecological factors on the genetic diversity and selection pressures in potyvirus populations present in evergreen oak and riparian forests of the Iberian Peninsula at the genus-wide (macroevolutionary scale) level is investigated. (ii) The population genetic structure of the potyvirus population is analysed in order to understand the modes of virus speciation in the studied wild ecosystems.

\subsubsection{Genetic diversity of potyviruses}

Given that sequences of a sole potyvirus species (MeRV) were collected in evergreen oak forests, in this ecosystem genus-wide and species-specific analyses are equivalent. Thus, the genetic diversity at the genus Potyvirus level 
was studied only in riparian forests, and species-specific analyses of the ecological factors affecting MeRV population genetic diversity are presented in section 4.5. As the potyvirus populations of riparian forests were genetically structured according to geographical location (see section 4.4.4 for details), evolutionary parameters (i.e., genetic diversity and selection pressures parameters) were quantified for potyvirus populations at each location. Both the partial Nlb and the CP genes sequence datasets were used. The following parameters were considered as measures of genetic diversity: the number of haplotypes $(H)$, the haplotype diversity $\left(H_{d}\right)$, the average nucleotide genetic diversity $(\pi)$, and the number of synonymous $\left(d_{S}\right)$ and non-synonymous $\left(d_{N}\right)$ substitutions per site (Table 4.30). The ratio between the latter two parameters was used as a measure of selection pressures in the virus population (Table 4.30).

When the partial Nlb sequence data set was used, potyvirus population haplotype number, haplotype diversity, genetic diversity and number of nonsynonymous and synonymous substitutions per site varied between locations $\left(\mathrm{H}=3-9 ; \mathrm{H}_{d}=0.733-0.933 ; \pi=0.012-0.231, d_{N}=0.000-0.198 ; d_{S}=0.128-0.808\right)$. The virus populations at El Escorial and Palazuelos had the highest values in all genetic diversity parameters, and Pollos the lowest (Table 4.30). In addition, the potyvirus populations in the four locations monitored were under purifying selection $\left(d_{N} / d_{S}<1\right)$. Purifying selection was weakest in the virus population of EI Escorial, and strongest in the virus population of Pollos (Table 4.30).

Data obtained using the CP gene sequence could be only obtained for two locations: Pollos and Palazuelos. For El Escorial and Santa Cruz de Retamar, less that three sequences were available and values could not be determined (Table 4.30). Potyvirus population haplotype number, haplotype diversity, genetic diversity and number of non-synonymous and synonymous substitutions per site varied between locations ( $H=3-9 ; H_{d}=0.978-1.000$; $\left.\Pi=0.020-0.029, d_{N}=0.015-0.019 ; d_{s}=0.022-0.226\right)$, but in a much narrower interval than for the Nlb (Table 4.30). The potyvirus population at Palazuelos had the higher value for all the estimated parameters (Table 4.30). Analyses also indicated that the CP gene was generally under purifying selection, this being much stronger in the virus population of Palazuelos than in the population of Pollos (Table 4.30). 
Table 4.30. Partial Nlb gene- and CP gene-based number of haplotypes $(H)$, haplotype diversity $\left(H_{d}\right)$, genetic diversity $(\pi)$, number of non-synonymous and synonymous substitutions $\left(d_{N}\right.$ and $d_{S}$ ) per site and $d_{N} / d_{S}$ ratio of genus-wide potyvirus populations in riparian forest locations.

\begin{tabular}{cccccccc}
\hline Location & $\mathbf{N}$ & $\mathbf{H}$ & $\mathbf{H}_{\mathbf{d}} \pm \mathbf{S E}$ & $\boldsymbol{\pi} \pm \mathbf{S E}$ & $\boldsymbol{d}_{\boldsymbol{N}} \pm \mathbf{S E}$ & $\boldsymbol{d}_{\boldsymbol{s}} \pm \mathbf{S E}$ & $\boldsymbol{d}_{\boldsymbol{N}} / \boldsymbol{d}_{\boldsymbol{s}} \pm \mathrm{SE}$ \\
\hline $\boldsymbol{N l b}$ & & & & & & & \\
$\mathrm{ESC}$ & 11 & 8 & $0.927 \pm 0.066$ & $0.231 \pm 0.038$ & $0.198 \pm 0.039$ & $0.738 \pm 0.092$ & $0.269 \pm 0.102$ \\
$\mathrm{PAL}$ & 16 & 9 & $0.933 \pm 0.035$ & $0.216 \pm 0.031$ & $0.163 \pm 0.036$ & $0.808 \pm 0.079$ & $0.202 \pm 0.086$ \\
$\mathrm{POL}$ & 13 & 6 & $0.833 \pm 0.071$ & $0.012 \pm 0.004$ & $0.000 \pm 0.000$ & $0.128 \pm 0.038$ & $0.000 \pm 0.038$ \\
$\mathrm{SCR}$ & 6 & 3 & $0.733 \pm 0.155$ & $0.209 \pm 0.041$ & $0.139 \pm 0.042$ & $0.535 \pm 0.078$ & $0.261 \pm 0.088$ \\
$\boldsymbol{C P}$ & & & & & & & \\
$\mathrm{ESC}$ & 2 & 2 & $\mathrm{ND}$ & $\mathrm{ND}$ & $\mathrm{ND}$ & $\mathrm{ND}$ & $\mathrm{ND}$ \\
$\mathrm{PAL}$ & 10 & 9 & $0.978 \pm 0.054$ & $0.029 \pm 0.004$ & $0.019 \pm 0.006$ & $0.226 \pm 0.029$ & $0.086 \pm 0.030$ \\
$\mathrm{POL}$ & 3 & 3 & $1.000 \pm 0.272$ & $0.020 \pm 0.005$ & $0.015 \pm 0.007$ & $0.022 \pm 0.010$ & $0.650 \pm 0.018$ \\
$\mathrm{SCR}$ & 1 & 1 & $\mathrm{ND}$ & $\mathrm{ND}$ & $\mathrm{ND}$ & $\mathrm{ND}$ & $\mathrm{ND}$ \\
\hline $\mathrm{N}$ & & &
\end{tabular}

$\mathrm{N}$ : number of sequences; SE: standard error; ND: Not determined.

\subsubsection{Analysis of recombination and nucleotide substitution rates of potyvirus populations}

To investigate which were the mechanisms contributing to the generation of genetic diversity of the potyvirus populations present in riparian forests, recombination and nucleotide substitution rates were estimated.

Inter and intra-species recombination events in the CP gene and in the partial Nlb gene sequences were analysed. These analyses were done using only the potyvirus sequences obtained in this thesis (Appendix 1, Table A1.4), and also including reference sequences from all known potyviruses (Appendix 1, Table A1.5). No recombination events were detected in any of the analyses, either between isolates of the same viral species or between isolates of different species. This result indicates that, at least for the analyzsd regions of the potyvirus genome, the main mechanisms generating genetic diversity was mutation. Thus, nucleotide substitution rates at the genus level were estimated for potyviruses of riparian forests.

The presence of temporal signal in the data utilized to estimate nucleotide substitution rates is essential to the accurate estimation of such substitution rates. Thus, the strength of temporal signal in the potyvirus partial $\mathrm{NIb}$ and the CP sequence datasets was first assessed. To do so, the BEAST 
analyses described below were repeated on data sets in which sampling times were randomized so that temporal structure was disrupted. Runs for randomized data were repeated 10 times (see section 3.3.2.1 of Materials and Methods). The mean and 95\% HPDs of the substitution rate estimates for the randomized data were then compared with those obtained from the real data. In all cases, estimates of the mean substitution rate differed by at least one order of magnitude between the real and randomized data sets (Figure 4.23). Also, the $95 \%$ HPD values for all of the randomized controls excluded the mean substitution rates estimated for the real data, indicating that they are significantly different. In addition, the lower 95\% HPD values in the randomized data sets differed by at least two orders of magnitude from the corresponding mean estimates, and hence strongly indicated insufficient temporal structure. Far narrower 95\% HPD results were observed for the real data. Hence, the sequence data analysed here contain sufficient temporal structure for reliable estimation.

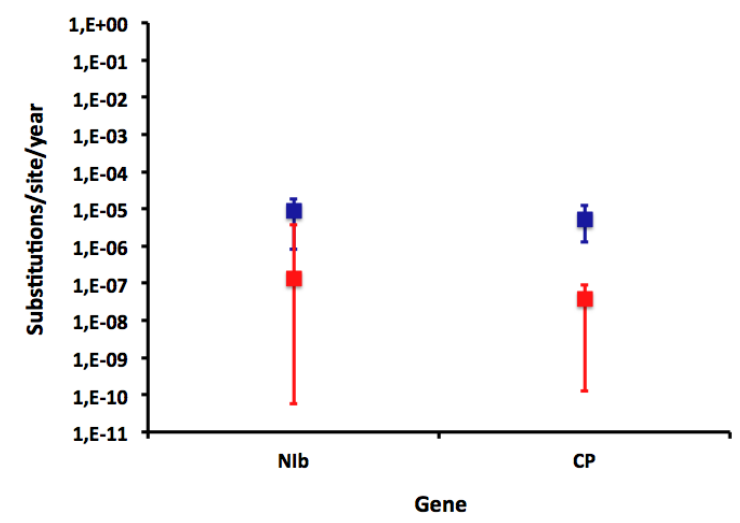

Figure 4.23. Estimates of the rate of nucleotide substitutions per site in the partial $\mathrm{Nlb}$ and the $\mathrm{CP}$ genes sequence data sets, using the real (blue squares) and randomized (red squares) dates of isolation, with $95 \%$ HPD values shown.

Because sampling time spanned only three sampling cycles, rates of nucleotide substitutions per site per day (subs/site/day) were estimated. Nucleotides substitution rates based on the partial NIb sequence were of 9.01 $\times 10^{-6}$ subs/site/day (95\% HPD: $8.22 \times 10^{-7}-7.19 \times 10^{-5}$ ). Substitution rates based on the CP gene were in the same range: $5.35 \times 10^{-6}$ subs/site/day $(95 \%$ HPD: $\left.1.27 \times 10^{-6}-1.22 \times 10^{-5}\right)$. These values roughly corresponded to $3.29 \times 10^{-3}$ subs/site/year (95\% HPD: 3.00 $\times 10^{-4}-2.62 \times 10^{-2}$ ), and $1.95 \times 10^{-4}$ subs/site/year (95\% HPD: $\left.4.63 \times 10^{-5}-4.45 \times 10^{-4}\right)$, for the NIb and the CP genes, respectively. 
Table 4.3.1. Average values of potyvirus prevalence and of ecological factors measured in riparian forests locations.

\begin{tabular}{|c|c|c|c|c|c|c|c|c|c|c|c|c|c|}
\hline Season & Location ${ }^{1}$ & Prevalence $^{2}$ & $\begin{array}{c}\text { Relative } \\
\text { abundance }\end{array}$ & $\begin{array}{c}\text { Plant } \\
\text { density }\end{array}$ & V & $\mathbf{S}$ & $T_{\max }$ & $\mathbf{T}_{\min }$ & $T_{\text {mean }}$ & $\mathbf{R} H_{\max }$ & $\mathrm{RH}_{\min }$ & $\mathbf{R} \mathbf{H}_{\text {mean }}$ & Rainfalls \\
\hline \multicolumn{14}{|l|}{ Sampling cycle 1} \\
\hline Summer 2013 & ESC & 0.00 & 9.50 & 4.08 & 0.38 & 29.00 & 32.95 & 7.55 & 20.43 & 88.75 & 13.50 & 39.50 & 159.00 \\
\hline Autumn 2013 & ESC & 0.00 & 8.50 & 3.29 & 0.10 & 28.00 & 27.07 & 2.43 & 14.77 & 99.67 & 19.00 & 59.00 & 434.67 \\
\hline Spring 2014 & ESC & 7.69 & 7.00 & 3.46 & 0.13 & 34.00 & 27.07 & 2.47 & 13.43 & 98.67 & 18.67 & 50.00 & 431.00 \\
\hline Summer 2013 & PAL & 4.35 & 18.00 & 5.95 & 0.08 & 31.00 & 31.10 & 5.58 & 18.33 & 94.25 & 14.25 & 46.50 & 216.00 \\
\hline Autumn 2013 & PAL & 8.33 & 40.70 & 6.82 & 0.01 & 32.00 & 25.93 & 0.07 & 13.07 & 98.67 & 17.00 & 63.67 & 277.33 \\
\hline Spring 2014 & PAL & 0.00 & 13.50 & 5.29 & 0.08 & 26.00 & 24.27 & 0.97 & 11.90 & 99.67 & 15.67 & 57.67 & 349.00 \\
\hline Summer 2013 & POL & 66.67 & 3.00 & 6.98 & 0.11 & 32.00 & 32.48 & 6.60 & 18.68 & 96.25 & 14.25 & 49.00 & 222.50 \\
\hline Autumn 2013 & POL & - & 0.00 & 0.00 & 0.10 & 32.00 & 26.30 & -0.80 & 12.90 & 99.00 & 31.50 & 65.50 & 299.00 \\
\hline Spring 2014 & POL & 66.67 & 1.00 & 13.15 & 0.13 & 35.00 & 25.50 & 1.23 & 12.67 & 99.00 & 18.00 & 56.33 & 166.00 \\
\hline Summer 2013 & SCR & 18.18 & 8.54 & 5.75 & 0.02 & 29.00 & 35.30 & 9.85 & 23.13 & 95.00 & 14.00 & 37.50 & 107.00 \\
\hline Autumn 2013 & SCR & 0.00 & 5.50 & 4.46 & 0.01 & 27.00 & 27.37 & 5.10 & 16.60 & 99.00 & 23.00 & 60.33 & 419.33 \\
\hline Spring 2014 & SCR & 33.33 & 3.00 & 5.09 & 0.06 & 30.00 & 27.97 & 4.50 & 15.30 & 98.00 & 17.00 & 52.67 & 315.33 \\
\hline \multicolumn{14}{|l|}{ Sampling cycle 2} \\
\hline Summer 2014 & ESC & 0.00 & 6.00 & 3.45 & 0.17 & 31.00 & 34.27 & 9.33 & 22.27 & 93.33 & 13.67 & 36.33 & 211.33 \\
\hline Autumn 2014 & ESC & 11.11 & 12.5 & 4.07 & 0.20 & 26.00 & 29.65 & 3.65 & 15.20 & 99.50 & 30.00 & 62.67 & 594.50 \\
\hline Spring 2015 & ESC & 22.22 & 13.5 & 3.95 & 0.15 & 32.00 & 27.53 & 1.27 & 13.70 & 96.00 & 16.33 & 53.00 & 230.67 \\
\hline Summer 2014 & PAL & 0.00 & 20.00 & 3.91 & 0.04 & 23.00 & 32.57 & 6.63 & 20.13 & 96.33 & 12.00 & 42.33 & 138.67 \\
\hline Autumn 2014 & PAL & 0.00 & 34.5 & 3.27 & 0.03 & 30.00 & 27.50 & 4.50 & 14.97 & 98.67 & 24.67 & 63.00 & 507.00 \\
\hline Spring 2015 & PAL & 19.61 & 28.00 & 3.54 & 0.07 & 21.00 & 24.70 & -0.73 & 11.87 & 98.33 & 15.33 & 61.00 & 232.00 \\
\hline Summer 2014 & POL & 14.29 & 2.00 & 5.44 & 0.13 & 35.00 & 34.57 & 7.33 & 20.47 & 93.33 & 12.67 & 44.33 & 80.00 \\
\hline Autumn 2014 & POL & 0.00 & 7.00 & 3.85 & 0.06 & 31.00 & 28.37 & 3.83 & 15.40 & 98.00 & 27.33 & 67.33 & 365.00 \\
\hline Spring 2015 & POL & 63.64 & 5.50 & 3.45 & 0.14 & 32.00 & 27.63 & -0.73 & 12.57 & 97.00 & 14.67 & 60.50 & 101.33 \\
\hline Summer 2014 & SCR & 0.00 & 8.50 & 3.66 & 0.00 & 34.00 & 36.13 & 13.00 & 24.20 & 95.00 & 14.00 & 36.33 & 45.33 \\
\hline Autumn 2014 & SCR & 0.00 & 2.50 & 8.41 & 0.06 & 27.00 & 29.90 & 7.90 & 17.40 & 99.00 & 23.00 & 67.67 & 1031.33 \\
\hline Spring 2015 & SCR & 14.29 & 8.00 & 12.92 & 0.01 & 17.00 & 29.60 & 3.90 & 15.80 & 98.00 & 17.00 & 55.00 & 319.33 \\
\hline
\end{tabular}

${ }^{1}$ Locations are designed with a three-letter code: ESC, El Escorial; POL, Pollos; SCR, Santa Cruz de Retamar.

${ }^{2}$ Percentage of infected plants relative to the total number of plants analysed from all host species present in each location.

$\mathrm{S}$ : Species richness

$\mathrm{V}$ : Plant biomass $\left(\mathrm{m}^{3}\right)$.

$T_{\max }, T_{\min }, T_{\text {mean }}$ : Average of monthly maximum, minimum and mean temperatures $\left({ }^{\circ} \mathrm{C}\right)$ in a season.

$\mathrm{RH}_{\max }, \mathrm{RH}_{\min }, \mathrm{RH}_{\text {mean }}$ : Average of monthly maximum, minimum and mean relative humidity (\%) in a season.

Rainfalls: Average of total rainfalls $(\mathrm{mm})$ in a season. 


\subsubsection{Analysis of ecological factors affecting the genus-wide potyvirus population genetic diversity}

The ecological factors (Table 4.31) affecting genus-wide potyvirus population $H_{d}, \pi, d_{N}, d_{S}$, and $d_{N} / d_{S}$ within riparian forests locations were analysed using multiple regression model selection analyses. The partial Nlb sequence dataset was used in this analysis. Note that, as for datasets used in sections 4.3 and 4.4, datasets utilized in the present section had much smaller sample size than those in section 4.2 and were normally distributed ( $W=0.92 ; P=0.391)$. The following factors were used as predictors: host plant density and relative abundance, species richness, plant biomass, temperature and relative humidity (minimal, maximal and average values), and rainfall in the sampled locations. Also, potyvirus prevalence was added as an additional predictor (Table 4.31). Potyvirus prevalence in each location was calculated as the percentage of infected plants relative to the total number of plants analysed from all host species present in each location. Host plant relative abundance and density were calculated accordingly. The best-ranked model explaining $\mathrm{H}_{\mathrm{d}}$ contained plant relative abundance and plant biomass as the most important predictors $\left(r=0.94 ; P=1 \times 10^{-5}\right)$. In this model plant relative abundance had higher relative importance than plant biomass (41\% and $29 \%$, respectively) (Table 4.32). Bivariate analyses indicated a positive association between these two predictors and $H_{d}(r=0.33 ; P=0.038)$ (Figure 4.24A and $\left.B\right)$. The best-ranked models predicting potyvirus genetic diversity $(\pi)$, and $d_{N}$, contained rainfalls and plant relative abundance as the most important predictors (relative importance ranged between $30 \%-44 \%)(r>0.85 ; P<0.006)$ (Table 4.32). Bivariate analysis showed a positive relationship between these predictors and the studied parameters $(r>0.33 ; P<0.039)$, although the association with plant relative abundance was only marginally significant $(r=0.27 ; P<0.067)$ (Figure 4.24C-F). The best-ranked models predicting $d_{S}$ contained again plant relative abundance and rainfalls as the most important predictors $\left(r=0.93 ; P=1 \times 10^{-5}\right)$ (Table 4.32), both factors showing a positive association with $d_{s}$ (Figure 4.24G and $\mathbf{H}$ ). The best-ranked models predicting $d_{N} / d_{S}(r=0.72 ; P=0.008)$ contained potyvirus prevalence and rainfalls as the most important predictors (Table 4.32). Bivariate analysis showed a negative relationship between $d_{N} / d_{S}$ and prevalence $(r=-$ 
$0.53 ; P=0.004)$, whereas the association with rainfalls was positive $(r=0.35$; $P=0.032$ ) (Figure $4.24 I$ and $\mathbf{J}$ ). No other combination of factors yielded a model that closely competed with the best-ranked one (Appendix 5, Table A5.1).

Table 4.32. Model selection analyses for potyvirus population haplotype diversity $\left(H_{d}\right)$, genetic diversity $(\pi)$, number of synonymous $\left(d_{S}\right)$ and non-synonymous $\left(d_{N}\right)$ substitutions per site, and selection pressures $\left(d_{N} / d_{S}\right)$. Model structures included host plant density, host relative abundance, plant species richness and plant biomass; and temperature, relative humidity and rainfall in the sampled locations (minimal, maximal and average values) as predictors, and season as covariate. Potyvirus prevalence was also included as predictor of virus evolution parameters. Best-ranked models are shown.

\begin{tabular}{|c|c|c|c|c|c|}
\hline Model structure & $r^{\dagger}$ & logLik & $\mathbf{A l C}^{\ddagger}$ & $\Delta_{\mathrm{i}} \S$ & $\boldsymbol{\omega}_{i}^{\mathbb{T}}$ \\
\hline \multicolumn{6}{|l|}{$H_{d}$} \\
\hline$P R A(41)+V(29)+H P D(11)+T_{\text {mean }}(10)+\mathrm{RH}_{\text {mean }}(9)$ & $0.94^{*}$ & 33.44 & -52.89 & 0.00 & 0.32 \\
\hline$P R A(47)+V(35)+T_{\text {mean }}(10)+\mathrm{RH}_{\text {mean }}(9)$ & $0.93^{*}$ & 32.06 & -52.13 & 0.76 & 0.22 \\
\hline $\mathrm{PRA}(40)+\mathrm{V}(28)+\mathrm{HPD}(11)+\mathrm{T}_{\text {mean }}(9)+\mathrm{RH}_{\text {mean }}(8)+\mathrm{RFA}(3)$ & $0.95^{\star}$ & 34.01 & -52.03 & 0.86 & 0.21 \\
\hline \multicolumn{6}{|l|}{$\pi$} \\
\hline$R A F(36)+P R A(32)+S(16)+R H_{\text {mean }}(12)+T_{\text {mean }}(4)$ & $0.85^{\star}$ & 24.46 & -34.91 & 0.00 & 0.33 \\
\hline $\mathrm{PRE}(36)+\mathrm{RAF}(23)+\mathrm{PRA}(18)+\mathrm{S}(12)+\mathrm{RH}_{\text {mean }}(7)+\mathrm{T}_{\text {mean }}(4)$ & $0.87^{\star}$ & 25.11 & -34.23 & 0.68 & 0.24 \\
\hline $\mathrm{RAF}(33)+\mathrm{PRA}(29)+\mathrm{S}(18)+\mathrm{RH}_{\text {mean }}(11)+\mathrm{HPD}(5)+\mathrm{T}_{\text {mean }}(4)$ & $0.86^{*}$ & 24.87 & -33.74 & 1.17 & 0.18 \\
\hline \multicolumn{6}{|l|}{$d_{N}$} \\
\hline $\mathrm{RAF}(44)+\mathrm{PRA}(30)+\mathrm{RH}_{\text {mean }}(11)+\mathrm{S}(10)+\mathrm{T}_{\text {mean }}(5)$ & $0.87^{\star}$ & 27.52 & -41.04 & 0.00 & 0.16 \\
\hline $\mathrm{RAF}(40)+\mathrm{PRA}(26)+\mathrm{S}(11)+\mathrm{RH}_{\text {mean }}(10)+\mathrm{HPD}(8)+\mathrm{T}_{\text {mean }}(5)$ & $0.88^{*}$ & 28.52 & -41.03 & 0.01 & 0.16 \\
\hline $\mathrm{PRE}(36)+\mathrm{RAF}(28)+\mathrm{PRA}(17)+\mathrm{S}(7)+\mathrm{T}_{\text {mean }}(6)+\mathrm{RH}_{\text {mean }}(6)$ & $0.88^{*}$ & 28.51 & -41.02 & 0.02 & 0.16 \\
\hline \multicolumn{6}{|l|}{$d_{s}$} \\
\hline $\mathrm{PRA}(45)+\mathrm{RAF}(25)+\mathrm{S}(9)+\mathrm{HPD}(8)+\mathrm{RH}_{\text {mean }}(8)+\mathrm{T}_{\text {mean }}(5)$ & $0.93^{*}$ & 15.54 & -15.08 & 0.00 & 0.16 \\
\hline$P R E(32)+P R A(29)+R A F(17)+S(6)+T_{\text {mean }}(6)+\mathrm{RH}_{\text {mean }}(5)+H P D(5)$ & $0.94^{*}$ & 16.51 & -15.02 & 0.07 & 0.15 \\
\hline$P R E(35)+P R A(31)+R A F(18)+R_{\text {mean }}(6)+S(5)+T_{\text {mean }}(5)$ & $0.93^{*}$ & 15.44 & -14.89 & 0.19 & 0.14 \\
\hline \multicolumn{6}{|l|}{$d_{N} / d_{s}$} \\
\hline PRE (49)+RAF (39)+V (12) & $0.72^{*}$ & 17.85 & -25.69 & 0.00 & 0.10 \\
\hline$R A F(44)+S(20)+P R A(19)+R H_{\text {mean }}(13)+T_{\text {mean }}(4)$ & $0.79^{*}$ & 19.64 & -25.28 & 0.41 & 0.08 \\
\hline PRE (44)+RAF (37)+V (14)+HPD (5) & $0.74^{*}$ & 18.50 & -25.69 & 0.69 & 0.07 \\
\hline
\end{tabular}

${ }^{*}$ The relative importance (\%) of each predictor variable is shown in parenthesis.

${ }^{\dagger}$ Correlation coefficient. Asterisks indicate significant correlations $(P<0.05)$.

¥Akaike’s Information Criterion.

$\S \Delta_{i}$, is the difference between the AIC of a given model and that of the best-ranked model, and quantifies how models compete (best-ranked model: $\Delta_{i}=0$; substantial empirical support: $\Delta_{i}=1-2$; considerable less support: $\Delta_{i}=2-7$; and no support; $\Delta_{i} 10$ ) (Burnham and Anderson, 2002).

"AIC model weight as $\omega_{i}=\exp \left(-0.5 \Delta_{i}\right) / \Sigma \exp \left(-0.5 \Delta_{i}\right)$. The larger the $\omega$, the greater the likelihood of the model relatively to the competing models. Maximum $\omega,=1$.

PRA: Plant relative abundance; V: Plant biomass; HPD: Host plant density; Tmean: Average temperature; $\mathrm{RH}_{\text {mean: }}$ Average relative humidity; RAF: Rainfalls; PRE: Prevalence; S: Species richness. 
A

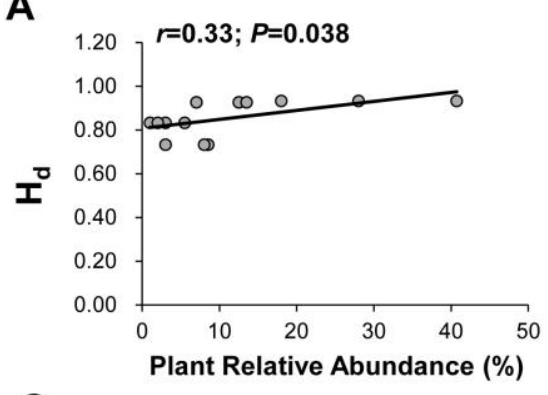

C

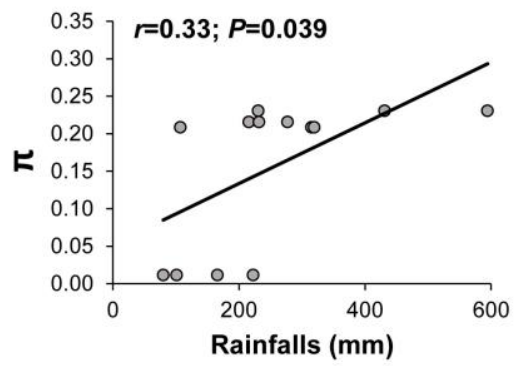

E

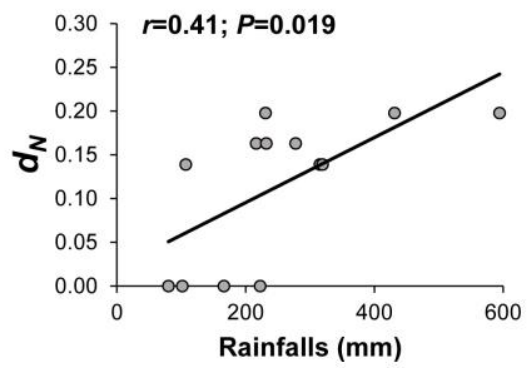

G

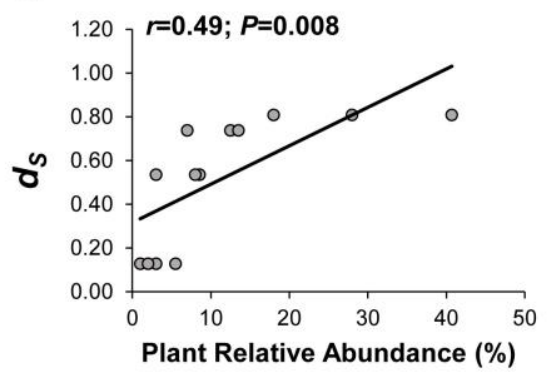

I

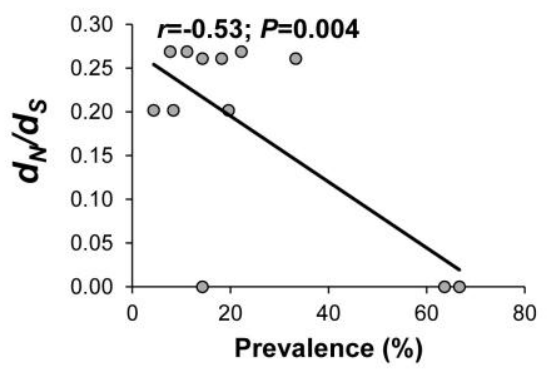

B

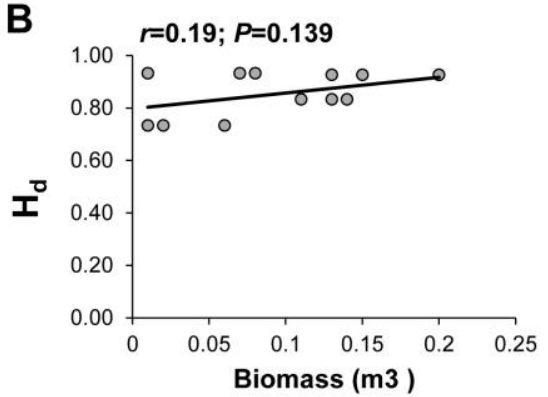

D

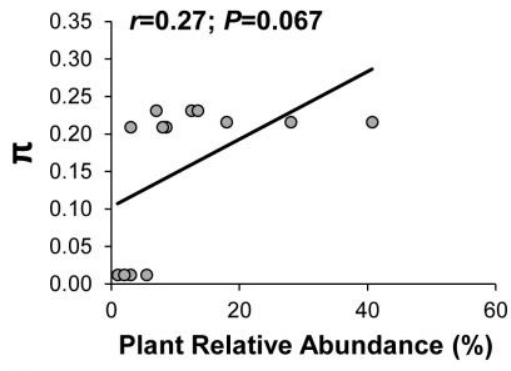

F

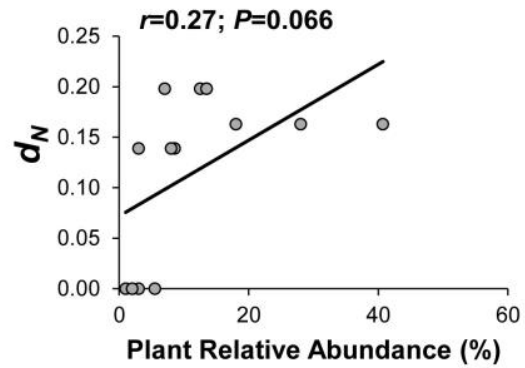

H

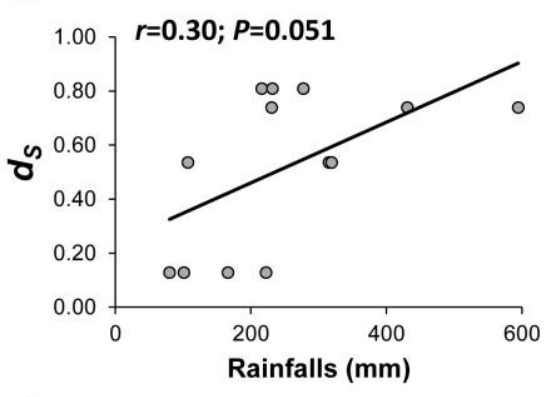

J

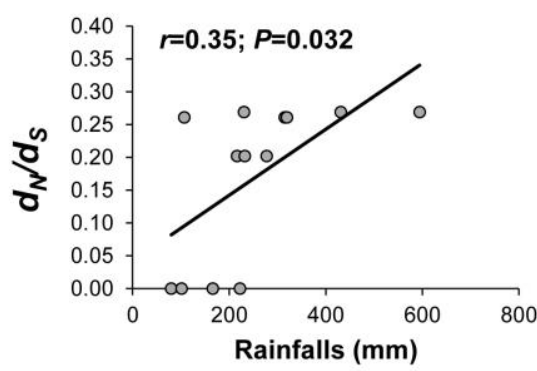

Figure 4.24. Bivariate relationships between potyvirus population evolutionary parameters and the best predictors. The associations between potyvirus population haplotype diversity (A and $B)$, population genetic diversity ( $C$ and $D$ ), number of non-synonymous ( $E$ and $F$ ) and synonymous mutations per site $(G$ and $H)$ and overall selection pressures $d_{N} / d_{s}(I$ and $J)$ and best predictors are represented. 
It is also important to indicate that models competing with the best ranked one that explained $d_{N}$ and $d_{S}$ contained potyvirus prevalence as the most important predictor (relative importance ranged between 32\% and 36\%) (Table 4.32). Potyvirus prevalence showed a negative association with both $d_{N}$ and $d_{S}(r-0.60 ; P<0.002)$ (Figure 4.25A and $\mathrm{B}$ ).

A

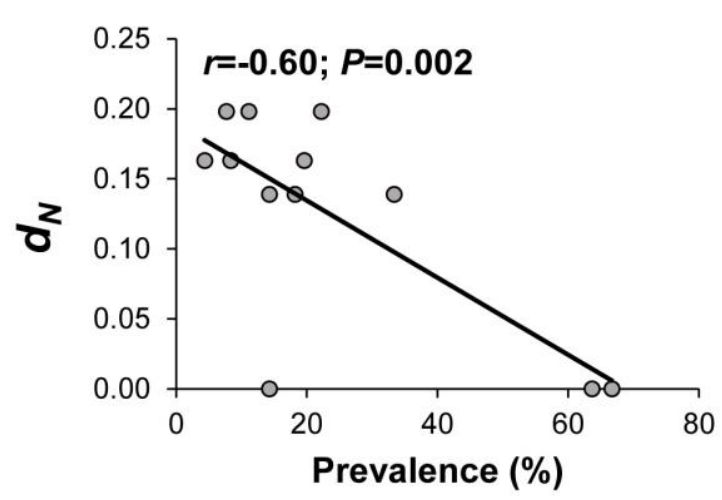

B

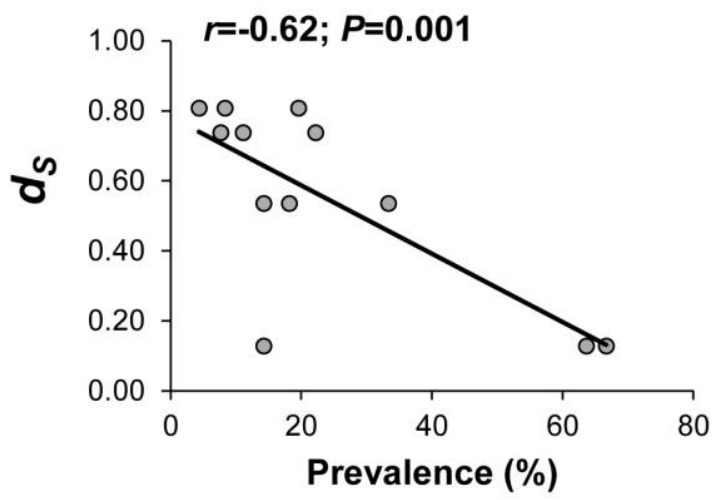

Figure 4.25. Bivariate relationships between potyvirus population evolutionary parameters and the best predictors in models competing with the best-ranked one. The associations of potyvirus population $d_{N}(\mathrm{~A})$, and population $d_{S}(\mathrm{~B})$ with potyvirus prevalence are represented.

\subsubsection{Analysis of potyvirus speciation modes}

To determine the respective roles of allopatric versus sympatric speciation, we assessed the strength of clustering by host species and location of sample origin within the interspecific potyvirus phylogeny. If allopatric speciation were the dominant process, we would expect no significant association between phylogeny and host species, and perhaps between phylogeny and geography. In contrast, if sympatric speciation were the most important macroevolutionary process in these data, we would expect a significant association between phylogeny and both the host species and the location of sampling (Kitchen et al., 2011).

The population genetic structure of the potyvirus species according to geographical location and host of origin of each isolate was analysed through association analyses. The degree of association between the geographical origin of each sequenced isolate and the host species, and the position of the corresponding Nlb sequence in the phylogeny was investigated using Maximum-likelihood trees (Figures 4.26 and 4.27), and estimating the 
Association Index (Al), Parsimony Score (PS) and Monophyletic Clade Size (MC) statistics. These analyses allowed exploring the modes of potyvirus speciation in the two ecosystems considered in this thesis.

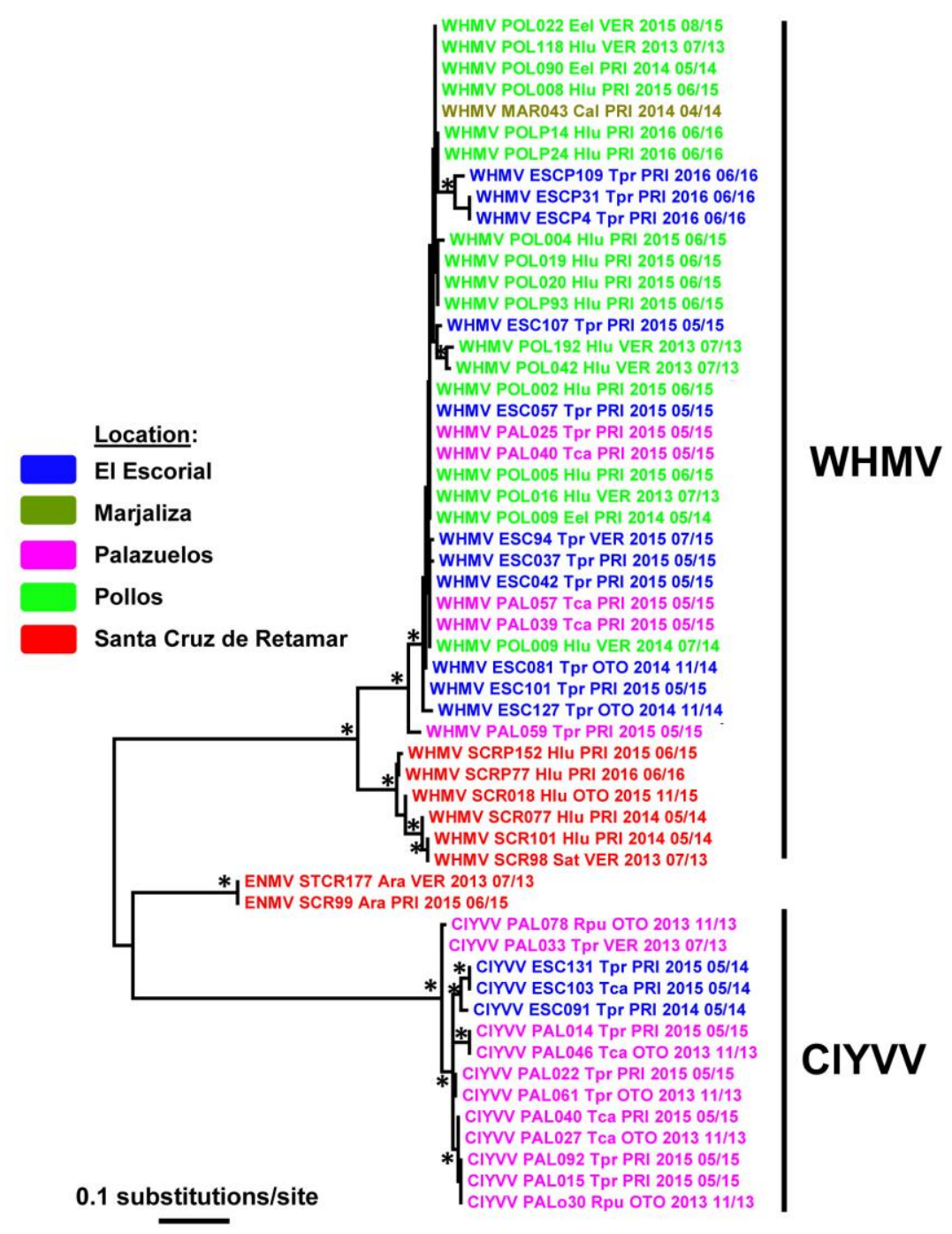

Figure 4.26. Maximum-likelihood phylogeny based on the Nlb region representing the association between potyvirus phylogeny and geographical location. Asterisks indicates nodes with a bootstrap support $\geq 0.90$.

Both the AI and PS statistics revealed a significant association between the interspecies phylogeny of the Potyvirus and the plant host each virus species naturally infects $(P<0.01)$; hence, virus species that have the same geographical origin tend to cluster together on the tree (Figure 4.26). Similarly, a statistically significant association was observed between the potyvirus phylogeny and the host that each virus infects $(P<0.01$ in both tests) (Figure 
4.27), such that virus species that infect plant hosts in the same geographic location tend to cluster together. Together, these data suggest that viral speciation tends to occur within the same host species and in the same geographic area, which is compatible with genetic diversification through sympatric speciation.

Association analyses were also done using the CP sequence dataset with comparable results (Appendix 5, Figure A5.1 and A5.2).

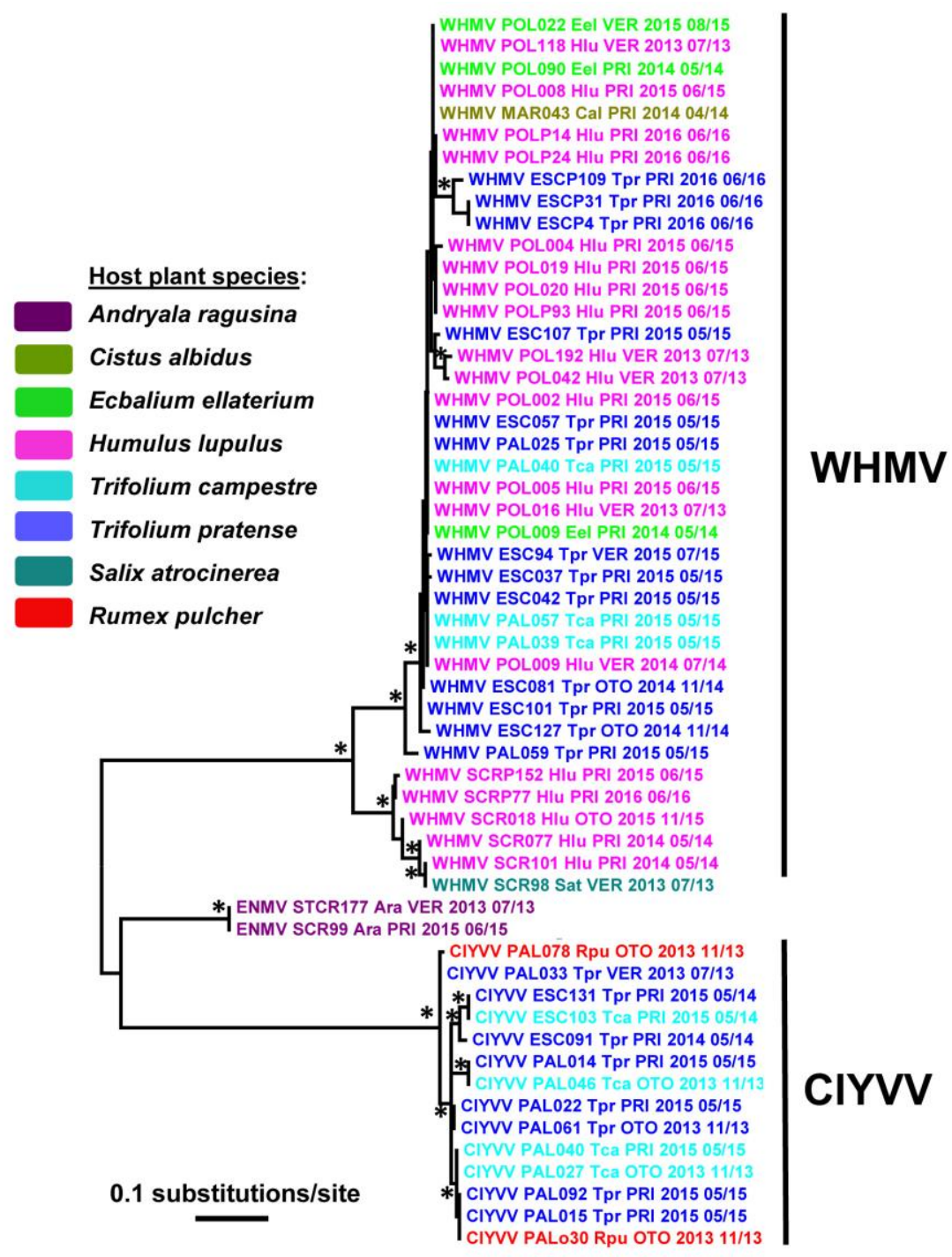

Figure 4.27. Maximum-likelihood phylogeny based on the Nlb region representing the association between potyvirus phylogeny and host plant species. Asterisks indicates nodes with a bootstrap support $\geq 0.90$. 


\subsection{Analysis of potyvirus species-specific genetic diversity in wild ecosystems in the Iberian Peninsula}

If the ecological factors that shape virus prevalence differ between specialist and generalists, the ecological determinants of virus population genetic diversity could also vary according to the virus host range (e.g. Hillung et al., 2015; Cuevas et al., 2016). Phylogenetic analyses in RNA viruses also suggest that the virus host range affects the speciation mode at the microevolutionary scale (Kitchen et al., 2011). However, the interplay between ecological factors, virus host range and population genetic diversity/structure has been seldom analysed in wild ecosystems.

These questions are addressed in this section by focusing in two of the virus species identified in evergreen oak and riparian forests: MeRV and WHMV. As described in previous sections, in the analysed ecosystems the first is a specialist, whereas the second is a generalist virus.

\subsubsection{Analysis of the population genetic diversity of a specialist potyvirus in wild ecosystems of the Iberian Peninsula}

Given that MeRV was identified as a new potyvirus species, the virus was genetically and biologically characterized prior to analysing the effect of ecological factors in the genetic diversity of MeRV populations.

\subsubsection{Analysis of the complete nucleotide sequence of MeRV}

The MeRV genomic sequence was obtained by NGS sequencing and confirmed by RT-PCR. The virus full-length genome sequence consists of 9,560nt excluding the 3' terminal poly(A) tail. As for all members of the genus Potyvirus, the MeRV-ParP17 genome has a single ORF encoding a polyprotein of 3,077 amino acids with an estimated molecular weight of $350 \mathrm{kDa}$. This ORF encodes all typical proteins, consensus cleavage sites and conserved catalytic domains present in the potyviruses (Figure 4.28). 


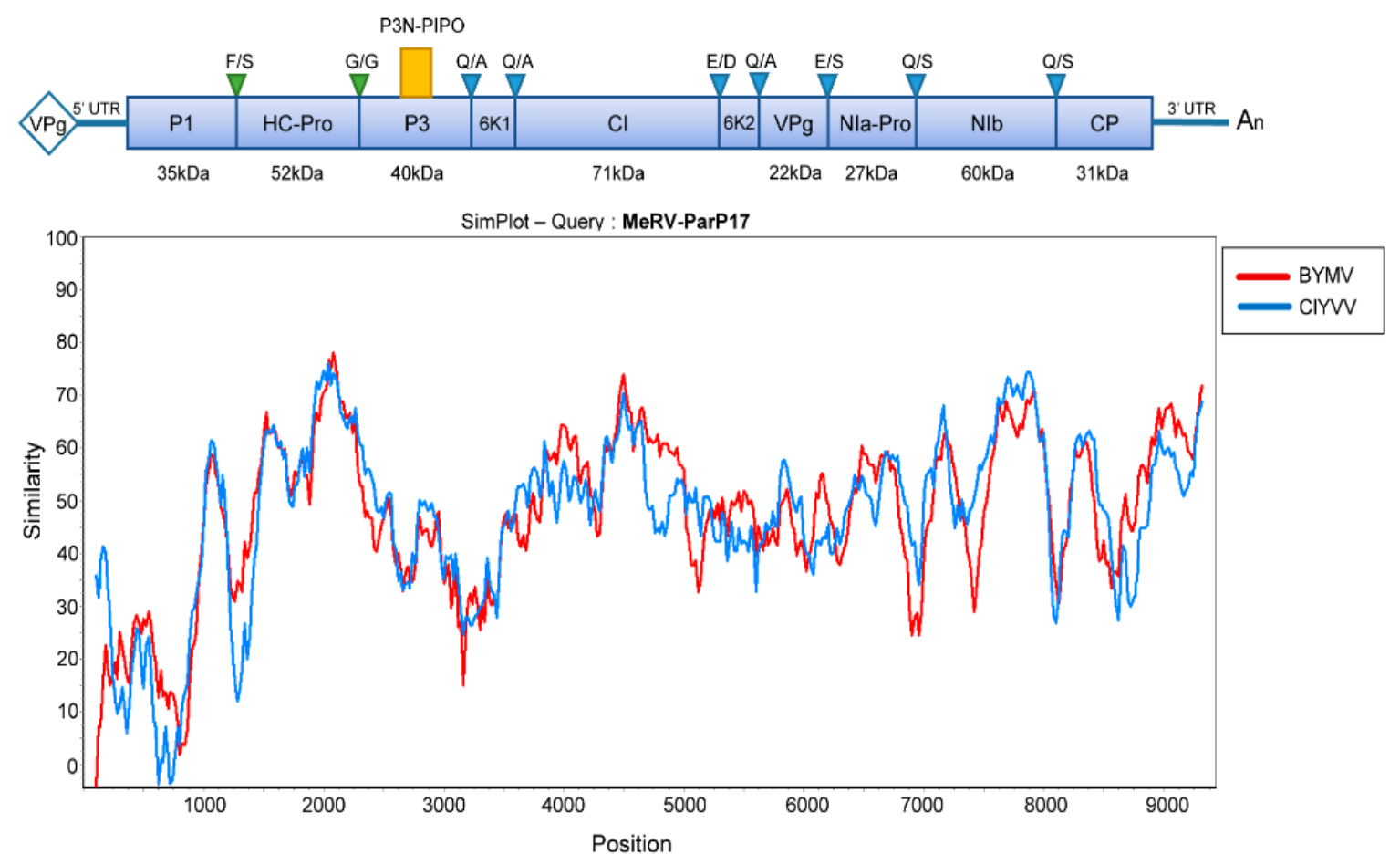

Figure 4.28. Schematic representation of the genomic organization of MeRV and of the nucleotide identity of the virus with the phylogenetically closer species. The position of each gene is indicated in relation to the sequence of the isolate MeRV-ParP17. The blue box represents the polyprotein ORF. The alternate P3N-PIPO ORF is depicted by the small yellow box above the main reading frame. The corresponding predicted molecular weights in kilodalton $(\mathrm{KDa})$ are indicated below each polyprotein cistron. Autocatalytic cleave sites of the HC-Pro and $\mathrm{P} 1$ gene products are indicated by green triangles, and Nla-Pro cleavage sites are marked by blue triangles. Conserved amino acids at each cleavage site according to Adams et al., 2005 are shown above each triangle. The panel below plots the nucleotide identities between MeRVParP17 genome and those of the phylogenetically nearest potyviruses: Bean yellow mosaic virus (BYMV) and Clover yellow vein virus (CIYVV). Each point plotted is the per cent identity within a sliding window of 200nt wide centred on the position plotted, with a step size between points of 20nt (Kimura two-parameter analysis).

The P1 protein, a serine protease, contains the strictly conserved catalytic triad His208- $\left(\mathrm{X}_{8}\right)$-Asp217- $\left(\mathrm{X}_{30}\right)-\mathrm{Ser}_{248}$ with the conserved GHSG $246-249$ motif around the active serine site. In the HC-Pro, the protease active site residues are located at Cys344, contained in the conserved sequence $\mathrm{GYCH}_{342-}$ 345 , and at His417. Also, a conserved cysteine-rich region was identified in the $\mathrm{N}$ terminal region of $\mathrm{HC}$-Pro stretching from Cys27 to Cys58. HC-Pro motifs

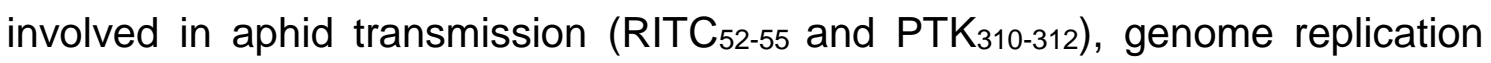
(IGN $152-154)$ and systemic movement (CCC $292-294)$ (Urcuqui-Inchima et al., 2001) are also conserved in MeRV-ParP17. The P3 cistron contains the P3N-PIPO 
ORF. The 5' end of this ORF starts with the highly conserved motif among potyviruses $\mathrm{G}_{2} \mathrm{~A}_{6}$ and it has a length of 70 codons, terminating in a UAG stop codon at nucleotides 672 to 674 of the P3 cistron. The cylindrical inclusion (Cl) protein cistron includes the conserved RNA helicase motifs located between residues 85 and 359, and the Nla contains the conserved His46, Asp 81 , Cys 151, His 167 cysteine protease catalytic tetrad. Conserved sequence motifs in RNAdependent-RNA polymerases of positive strand RNA viruses were also identified in the MeRV-ParP17 Nlb protein. Finally, the CP cistron contains a NAG motif, essential for aphid transmission in some potyviruses (Wylie et al., 2002), and located at residues 10-12 in the N-terminal region of the CP. The $\operatorname{Arg}_{170}$ and Asp $_{214}$ residues, involved in virion assembly and cell-to-cell movement (Dolja et al., 1994, 1995), are located in the conserved core region of the MeRV-ParP17 CP. The virus 5' untranslated region (UTR) is $149 \mathrm{nt}$ long, an average length for potyviruses (<200nt). The 3' UTR is $180 \mathrm{nt}$ long excluding the poly(A)-tail and is rich in AU segments, which is a common feature among potyviruses.

The complete nucleotide sequence of the MeRV-ParP17 polyprotein was aligned with those of available fully sequenced potyviruses ( $n=107$ ) (Appendix 1, Table A1.5). The percentage of nucleotide identity of the MeRV-ParP17 genome with that of the other potyviruses ranged from $50 \%$ to $66 \%$, showing highest identity with bean yellow mosaic virus (BYMV) (65\%) and clover yellow vein virus (CIYVV) (66\%). Accordingly, Bayesian phylogenetic trees revealed that MeRV-ParP17 clustered together with BYMV and CIYVV (Figure 4.29). Following the approach described by Mbanzibwa et al., 2011 SimPlot analyses were performed to compare the percentage of nucleotide identity between MeRV-ParP17 and BYMV/CIYVV across the whole genome (Figure 4.28). When each cistron was analysed individually, the MeRV-ParP17 P1 and P3 cistrons showed the lowest nucleotide identity with the same cistrons of BYMV and CIYVV (Figure 4.28). The remaining cistrons showed a sequence identity with the corresponding ones of BYMV and CIYVV ranging on average between $57 \%$ and $69 \%$. Equivalent results were obtained using the amino acid sequences. 


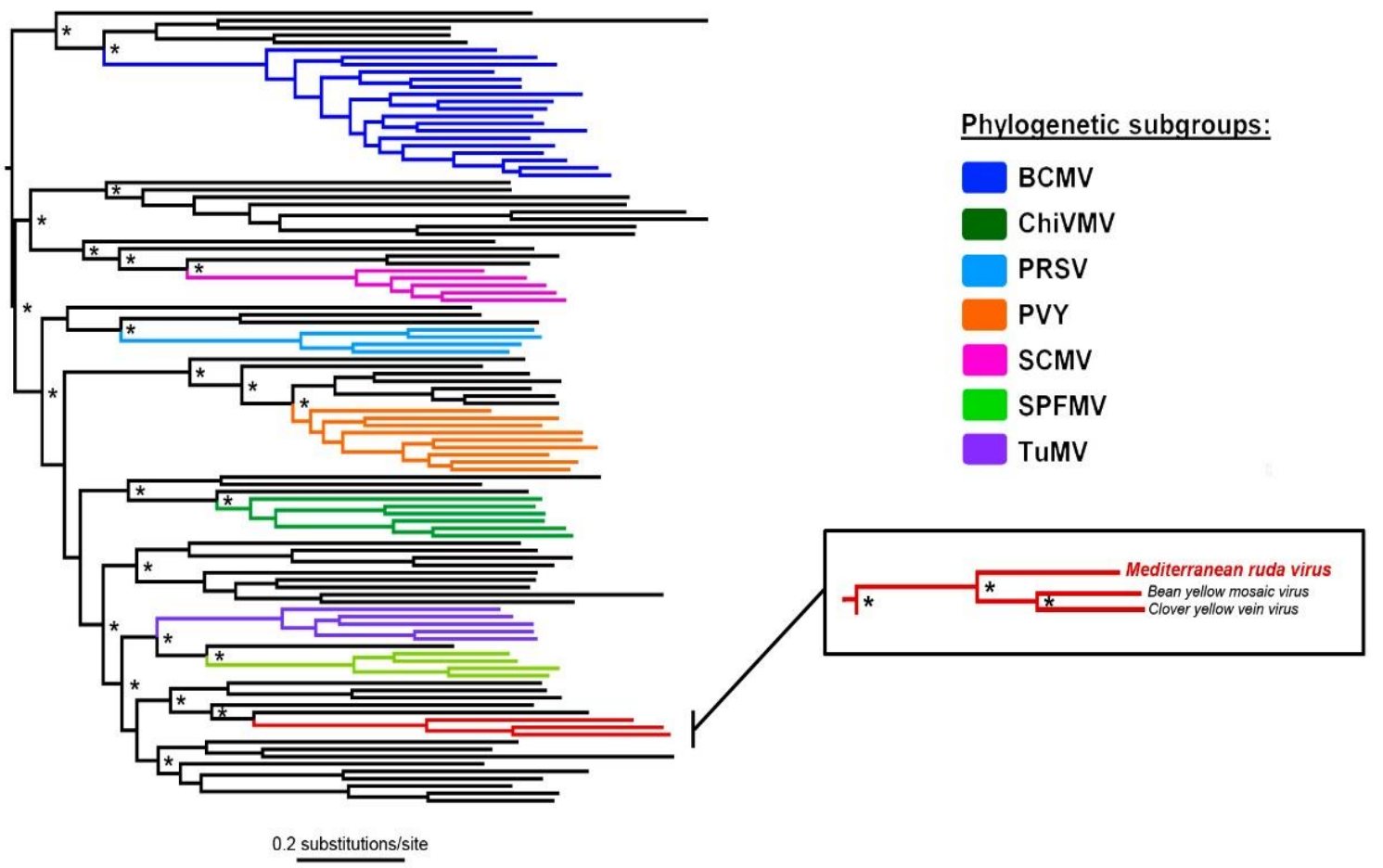

Figure 4.29. Bayesian phylogeny based on the coding nucleotide sequence of potyviruses. Asterisks indicate nodes with posterior probabilities of $\geq 0.90$. The tree is mid-point rooted. Branch lengths are drawn to a scale of nucleotide substitutions per site. Phylogenetic subgroups of potyviruses defined by Shukla et al., 1994 are used as reference and indicated in coloured branches. BCMV: Bean common mosaic virus; ChiVMV: Chilli veinal mottle virus; PRSV: Papaya ringspot virus; PVY: Potato virus Y; SCMV: Sugarcane mosaic virus; SPFMV: Sweet potato feathery mottle virus; TuMV: Turnip mosaic virus. Expansion of the phylogeny section contains the group of virus sequences in which MeRV is clustered.

\subsubsection{MeRV host range}

The MeRV-ParP17 host range was determined by inoculating purified virions of MeRV-ParP17 into 10 wild species belonging to seven botanical families, which represent the most abundant species in evergreen oak forests, and 13 plant species of six botanical families, including Cucurbitaceae and Solanaceae species that are commonly cultivated or found in the area surrounding the surveyed evergreen oak forests. The results are shown in Table 4.33.

None of the 10 wild species from evergreen oak forests were hosts of MeRV, except its original reservoir mountain rue (6/6 plants infected), in which virus infection was asymptomatic. Only three Solanaceae species were infected by MeRV-ParP17: N. benthamiana (5/5), C. annuum (4/6) and S. lycopersicum (6/6), infection in these three species causing mild mosaic symptoms. There was no variation in susceptibility across hosts $\left(X^{2}=0.81, P=0.668\right)$. 
Table 4.33. Host range and symptoms of MeRV-ParP17.

\begin{tabular}{|c|c|c|}
\hline 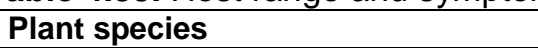 & Systemic infection $^{1}$ & Systemic symptoms \\
\hline \multicolumn{3}{|l|}{ Amaranthaceae } \\
\hline Gomphrena globosa & $0 / 6$ & - \\
\hline \multicolumn{3}{|l|}{ Asteraceae } \\
\hline Lactuca sativa & $0 / 6$ & - \\
\hline Santolina rosmarinifolia* & $0 / 6$ & - \\
\hline Taraxacum officinalis* & $0 / 6$ & - \\
\hline \multicolumn{3}{|l|}{ Boraginaceae } \\
\hline Anchusa azurea* & $0 / 7$ & - \\
\hline \multicolumn{3}{|l|}{ Chenopodiaceae } \\
\hline Chenopodium amaranticolor & $0 / 6$ & - \\
\hline Chenopodium quinoa & $0 / 5$ & - \\
\hline \multicolumn{3}{|l|}{ Cistaceae } \\
\hline Cistus ladanifer* & $0 / 6$ & - \\
\hline Cucurbitaceae & & - \\
\hline Cucumis melo & $0 / 5$ & - \\
\hline Cucumis sativus & $0 / 5$ & \\
\hline \multicolumn{3}{|l|}{ Fabaceae } \\
\hline Anthyllis vulneraria* & $0 / 5$ & - \\
\hline Lupinus angustifolius* & $0 / 6$ & - \\
\hline Phaseolus vulgaris & $0 / 5$ & - \\
\hline Pisum sativum & $0 / 6$ & - \\
\hline Vicia villosa* & $0 / 6$ & - \\
\hline \multicolumn{3}{|l|}{ Fagaceae } \\
\hline Quercus ilex* & $0 / 4$ & - \\
\hline \multicolumn{3}{|l|}{ Poaceae } \\
\hline Bromus rubens* & $0 / 6$ & - \\
\hline \multicolumn{3}{|l|}{ Rutaceae } \\
\hline Ruta montana & $6 / 6$ & Asymptomatic \\
\hline \multicolumn{3}{|l|}{ Solanaceae } \\
\hline Capsicum annuum (Dulce Italiano) & $4 / 6$ & Mild mosaic \\
\hline Nicotiana benthamiana & $5 / 5$ & Mild mosaic \\
\hline Nicotiana clevelandii & $0 / 5$ & - \\
\hline Nicotiana tabacum (Samsun) & $0 / 5$ & - \\
\hline Solanum lycopersicum & $6 / 6$ & Mild mosaic \\
\hline
\end{tabular}

\subsubsection{Analysis of MeRV population genetic diversity}

All the MeRV sequenced isolates were collected at El Pardo. The lack of isolates from other locations and/or hosts prevented to analyze the genetic structure of this virus population according to these factors. Hence, to test whether changes in ecological factors and MeRV prevalence across seasons affected the genetic diversity of the virus population, the CP sequence of 69 MeRV isolates from this location, collected along three growth cycles were utilized (Table 4.34). Using this sequence data set, the number of haplotypes $(H)$, the haplotype diversity $\left(H_{d}\right)$, the average nucleotide genetic diversity $(\pi)$, and the number of synonymous $\left(d_{S}\right)$ and non-synonymous $\left(d_{N}\right)$ substitutions per site of the MeRV population at each visit were estimated. MeRV population haplotype number, haplotype diversity, genetic diversity and number of nonsynonymous and synonymous substitutions per site greatly varied between 
seasons $\left(H=2-9 ; H_{d}=0.50-1.00 ; \quad \pi=0.001-0.007 ; \quad d_{N=0.001-0.006 ;} d_{S}=0.001\right.$ 0.053) (Table 4.34). The highest values in all genetic diversity parameters were obtained in the first and third sampling cycles. Averaged measures of $d_{N} / d_{S}$ indicated that MeRV populations were under purifying selection $\left(d_{N} / d_{S}<1\right)$ in the first and third sampling cycles, while neutral evolution seems to be responsible for the changes in MeRV in the second sampling cycle $\left(d_{N} / d_{S}=1\right)$. Note that this is due to the extremely high $d_{N} / d_{S}$ value in Spring 2015.

Table 4.34. Number of haplotypes $(H)$, haplotype diversity $\left(H_{d}\right)$, genetic diversity $(\pi)$, and number of non-synonymous and synonymous substitutions $\left(d_{N}\right.$ and $\left.d_{S}\right)$ per site and $d_{N} / d_{s}$ of the El Pardo MeRV population based on the CP gene.

\begin{tabular}{|c|c|c|c|c|c|c|c|}
\hline Season & $\mathbf{N}$ & $\mathbf{H}$ & $H_{d} \pm S E$ & $\pi \pm \mathrm{SE}$ & $d_{N} \pm S E$ & $d s \pm S E$ & $d_{N} / d s \pm \mathrm{SE}$ \\
\hline \multicolumn{8}{|l|}{ Sampling cycle 1} \\
\hline Summer 2013 & 7 & 6 & $0.952 \pm 0.036$ & $0.006 \pm 0.002$ & $0.005 \pm 0.004$ & $0.037 \pm 0.012$ & $0.135 \pm 0.009$ \\
\hline Autumn 2013 & 8 & 7 & $0.964 \pm 0.027$ & $0.007 \pm 0.002$ & $0.006 \pm 0.004$ & $0.050 \pm 0.016$ & $0.120 \pm 0.012$ \\
\hline Spring 2014 & 5 & 4 & $0.900 \pm 0.072$ & $0.004 \pm 0.002$ & $0.001 \pm 0.000$ & $0.028 \pm 0.010$ & $0.035 \pm 0.000$ \\
\hline Cycle ${ }^{1}$ & 20 & 16 & $0.974 \pm 0.014$ & $0.007 \pm 0.002$ & $0.015 \pm 0.007$ & $0.072 \pm 1 \times 10^{-5}$ & $0.097 \pm 0.011$ \\
\hline \multicolumn{8}{|l|}{ Sampling cycle 2} \\
\hline Summer 2014 & 9 & 6 & $0.889 \pm 0.030$ & $0.004 \pm 0.002$ & $0.002 \pm 0.002$ & $0.043 \pm 0.015$ & $0.047 \pm 0.002$ \\
\hline Autumn 2014 & 7 & 4 & $0.714 \pm 0.068$ & $0.006 \pm 0.002$ & $0.004 \pm 0.003$ & $0.022 \pm 0.009$ & $0.181 \pm 0.003$ \\
\hline Spring 2015 & 4 & 2 & $0.500 \pm 0.133$ & $0.001 \pm 0.001$ & $0.003 \pm 0.002$ & $0.001 \pm 0.000$ & $3.000 \pm 0.020$ \\
\hline Cycle & 20 & 12 & $0.932 \pm 0.020$ & $0.005 \pm 0.001$ & $0.009 \pm 0.020$ & $0.066 \pm 0.004$ & $1.076 \pm 0.020$ \\
\hline \multicolumn{8}{|l|}{ Sampling cycle 3} \\
\hline Summer 2015 & 4 & 4 & $1.000 \pm 0.089$ & $0.007 \pm 0.002$ & $0.006 \pm 0.004$ & $0.042 \pm 0.014$ & $0.143 \pm 0.004$ \\
\hline Autumn 2015 & 10 & 4 & $0.778 \pm 0.029$ & $0.004 \pm 0.001$ & $0.002 \pm 0.002$ & $0.045 \pm 0.014$ & $0.044 \pm 0.002$ \\
\hline Spring 2016 & 15 & 9 & $0.848 \pm 0.023$ & $0.006 \pm 0.002$ & $0.006 \pm 0.004$ & $0.053 \pm 0.014$ & $0.113 \pm 0.004$ \\
\hline Cycle & 29 & 16 & $0.931 \pm 0.016$ & $0.006 \pm 0.001$ & $0.018 \pm 0.009$ & $0.098 \pm 0.024$ & $0.100 \pm 0.009$ \\
\hline
\end{tabular}

\subsubsection{Analysis of ecological factors affecting MeRV population genetic diversity}

The ecological factors affecting MeRV population $\mathrm{H}_{\mathrm{d}}, \pi, d_{N}, d_{S}$ and $d_{N} d_{S}$ were analysed using multiple regression model selection analyses (Table 4.35). The same variables as for predicting potyvirus species-specific prevalence and adding MeRV prevalence as an additional predictor (data of predictor variables is in Section 4.3, Table 4.24) were utilized. The best-ranked model explaining $\mathrm{H}_{d}$ contained prevalence as the only predictor $(r=0.79 ; P=0.011)$ (Table 4.35). This model closely competed with that including MeRV prevalence and plant species richness as main predictors ( $r=0.73 ; P=0.055)$, virus prevalence having much higher relative importance than plant species richness ( $89.8 \%$ and $10.2 \%$, 
respectively). The model containing MeRV prevalence and host relative abundance $(r=0.70 ; P=0.046)$ also showed $\Delta_{i}<2$, with virus prevalence having again the highest relative importance (99.1\%) (Table 4.35). Bivariate analyses indicated a positive association between $\mathrm{H}_{d}$ and virus prevalence (Figure 4.30A).

Table 4.35. Model selection analyses for MeRV haplotype diversity $\left(H_{d}\right)$, genetic diversity $(\pi)$, number of synonymous $\left(d_{S}\right)$ and non-synonymous $\left(d_{N}\right)$ substitutions per site, and selection pressures $\left(d_{N} / d_{S}\right)$. Model structures included host plant density, host relative abundance, plant species richness and plant biomass; and temperature, relative humidity and rainfall in the sampled locations (minimal, maximal and average values) as predictors, and season as covariate. MeRV prevalence was also included as predictor of virus evolution parameters. Bestranked models are shown.

\begin{tabular}{|c|c|c|c|c|c|}
\hline Model structure $^{*}$ & $r^{\dagger}$ & logLik & AIC $\ddagger$ & $\Delta_{\mathrm{i}}^{\mathrm{s}}$ & $\omega_{i}^{\pi}$ \\
\hline \multicolumn{6}{|l|}{$H_{d}$} \\
\hline PRE (100) & $0.79^{*}$ & 7.60 & -9.21 & 0.00 & 0.47 \\
\hline PRE (90) + S (10) & $0.73^{*}$ & 7.19 & -8.38 & 0.83 & 0.30 \\
\hline PRE (99) + PRA (1) & $0.70^{*}$ & 8.22 & -7.67 & 1.51 & 0.22 \\
\hline \multicolumn{6}{|l|}{$\pi$} \\
\hline PRE (77) + S (23) & $0.74^{\star}$ & 35.62 & -63.23 & 0.00 & 0.47 \\
\hline PRE (100) & $0.64^{*}$ & 36.35 & -62.15 & 1.08 & 0.27 \\
\hline $\operatorname{PRE}(99)+\operatorname{RAF}(1)$ & 0.43 & 37.00 & -61.99 & 1.24 & 0.25 \\
\hline \multicolumn{6}{|l|}{$d_{N}$} \\
\hline PRE (80) + S (20) & 0.70 & 45.44 & -82.89 & 0.00 & 0.71 \\
\hline$S(100)$ & -0.65 & 45.54 & -81.09 & 1.80 & 0.29 \\
\hline \multicolumn{6}{|l|}{$d_{s}$} \\
\hline PRE (75) + S (25) & $0.82^{*}$ & 29.60 & -49.21 & 0.00 & 0.56 \\
\hline PRE (100) & $0.69^{*}$ & 29.72 & -47.43 & 1.78 & 0.23 \\
\hline $\operatorname{PRE}(72)+S(17)+\operatorname{RAF}(11)$ & 0.83 & 29.88 & -47.30 & 1.91 & 0.21 \\
\hline \multicolumn{6}{|l|}{$d_{N} / d_{s}$} \\
\hline PRE (85) + S (15) & $0.83^{*}$ & 30.34 & -50.68 & 0.00 & 0.56 \\
\hline PRE (78) + S (13) + RAF (9) & $0.84^{*}$ & 30.44 & -48.88 & 1.80 & 0.23 \\
\hline PRE (100) & $0.68^{*}$ & 30.12 & -48.75 & 1.93 & 0.21 \\
\hline
\end{tabular}

"The relative importance (\%) of each predictor variable is shown in parenthesis.

${ }^{\dagger}$ Correlation coefficient. Asterisks indicate significant correlations $(P<0.05)$.

${ }^{\ddagger}$ Akaike’s Information Criterion.

$\S \Delta_{i}$, is the difference between the AIC of a given model and that of the best-ranked model, and quantifies how models compete (best-ranked model: $\Delta_{i}=0$; substantial empirical support: $\Delta i=1-2$; considerable less support: $\Delta_{i}=2-7$; and no support; $\left.\Delta>10\right)$ (Burnham and Anderson, 2002).

"AIC model weight as $\omega_{i}=\exp \left(-0.5 \Delta_{i}\right) / \Sigma \exp \left(-0.5 \Delta_{i}\right)$. The larger the $\omega$, the greater the likelihood of the model relatively to the competing models. Maximum $\omega,=1$.

PRE: MeRV prevalence; S: Plant species richness; PRA: Plant relative abundance; RAF: Rainfalls. 
The model best explaining MeRV population genetic diversity $(\pi)$ contained MeRV prevalence and plant species richness as predictors $(r=0.74$; $P=0.022)$, prevalence having higher relative importance (77.2\%) (Table 4.35). This model closely competed with that containing MeRV prevalence as the sole predictor ( $r=0.64 ; P=0.042$ ) (Table 4.35 and Figure $4.30 \mathrm{~B}$ ), or this variable together with rainfall $(r=0.43 ; P=0.541)$. However, the later model did not show a significant association between the predictor(s) and the response variable (Table 4.35). None of the tested models accurately predicted $d_{N}(P \geq 0.106)$. On the other hand, the best-ranked models predicting $d_{S}$ and $d_{N} / d_{S}$ contained MeRV prevalence as predictor, solely ( $r \geq-0.68 ; P \leq 0.018$ ) (Figures $4.30 \mathrm{C}, \mathrm{D}$ ) or in combination with plant species richness and rainfall ( $r \geq 0.83 ; P<0.050$ ), MeRV prevalence having always much higher predictive power (relative importance $\geq 72 \%$ ) than the other two predictors (relative importance $\leq 25 \%$ ) (Table 4.35).
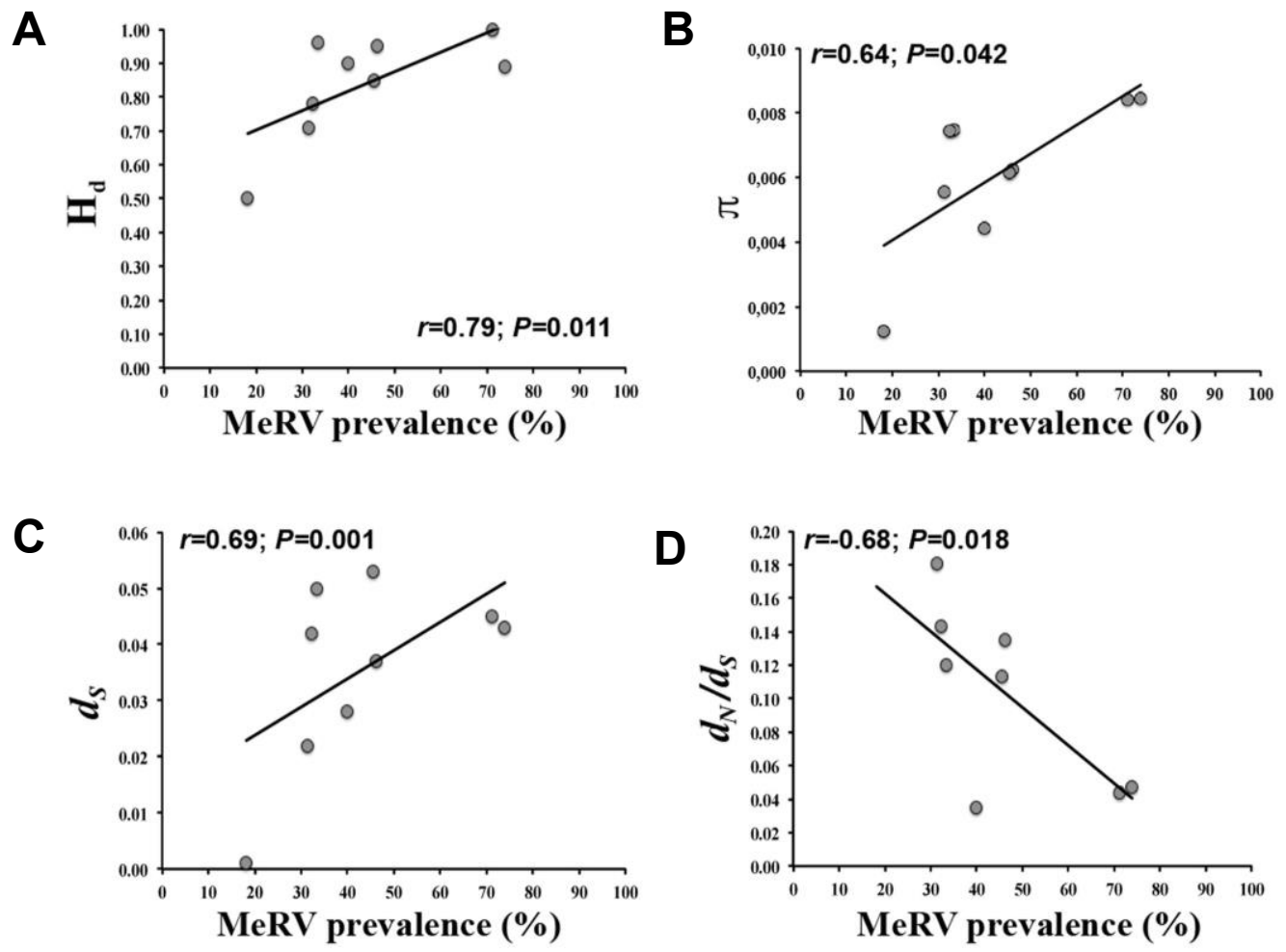

Figure 4.30. Bivariate relationships between MeRV population evolutionary parameters and the best predictor variable. In each case, best predictor variable was identified by model selection analyses. Regressions of MeRV population haplotype diversity (A), population genetic diversity (B), number of synonymous mutations per site (C) and overall selection pressures $d N / d s$ (D) on MeRV prevalence are represented.

Thus, overall, MeRV prevalence is the best predictor of the genetic diversity and selection pressures in the virus population. 


\subsubsection{Analysis of the population genetic diversity of a generalist potyvirus in wild ecosystems of the Iberian Peninsula}

\subsubsection{Analysis of WHMV population genetic diversity}

WHMV was detected in four riparian forest locations. Unfortunately, it was not possible to obtain enough number of CP sequences of WHMV isolates at each location to analyse the population genetic diversity. Hence, at odds with MeRV, analysis of WHMV population genetic diversity was performed using the partialNIb gene sequence of the 40 WHMV isolates collected along three sampling cycles. However, note that both genomic regions contain similar phylogenetic information (see section 3.2.2 and Apppendix 1, Table A1.3). As WHMV populations were genetically structured according to geographical location (see section 4.5.2.3 below), genetic diversity and selection pressures parameters were quantified at each location. As WHMV was not detected in every season during the monitored period, not enough sequences were obtained at each visit to perform seasonal estimates of genetic diversity parameters.

WHMV population haplotype number, haplotype diversity, genetic diversity and number of non-synonymous and synonymous substitutions per site varied between locations $\left(H=3-10 ; H_{d}=0.800-0.982 ; \pi=0.011-0.033\right.$, $\left.d_{N=0.000-0.066 ;} d_{S}=0.109-0.233\right)$. The WHMV populations at El Escorial and Santa Cruz de Retamar had the highest values in all genetic diversity parameters (Table 4.36). In addition, the WHMV populations in El Escorial and Palazuelos were under purifying selection $\left(d_{N} / d_{s}<1\right)$, which was stronger in the WHMV populations at Pollos and Santa Cruz de Retamar (Table 4.36).

Table 4.36. Number of haplotypes $(H)$, haplotype diversity $\left(H_{d}\right)$, genetic diversity $(\pi)$, number of non-synonymous and synonymous substitutions $\left(d_{N}\right.$ and $\left.d_{S}\right)$ per site and selection pressures $\left(d_{N} / d_{S}\right)$ of WHMV populations in riparian forests based on the partial NIb gene sequence.

\begin{tabular}{cccccccc}
\hline Location & $\mathbf{N}$ & $\mathbf{H}$ & $\mathbf{H}_{\mathbf{d}} \pm \mathbf{S E}$ & $\boldsymbol{\pi} \pm \mathbf{S E}$ & $\boldsymbol{d}_{\boldsymbol{N}} \pm \mathbf{S E}$ & $\boldsymbol{d}_{\boldsymbol{s}} \pm \mathbf{S E}$ & $\boldsymbol{d}_{\boldsymbol{N}} / \boldsymbol{d}_{\boldsymbol{s}} \pm \mathbf{S E}$ \\
\hline ESC & 11 & 10 & $0.982 \pm 0.046$ & $0.033 \pm 0.007$ & $0.066 \pm 0.017$ & $0.233 \pm 0.073$ & $0.285 \pm 0.072$ \\
$\mathrm{PAL}$ & 5 & 3 & $0.800 \pm 0.164$ & $0.016 \pm 0.005$ & $0.005 \pm 0.005$ & $0.109 \pm 0.035$ & $0.044 \pm 0.035$ \\
$\mathrm{POL}$ & 17 & 8 & $0.882 \pm 0.047$ & $0.011 \pm 0.003$ & $0.000 \pm 0.000$ & $0.150 \pm 0.046$ & $0.000 \pm 0.046$ \\
SCR & 6 & 5 & $0.933 \pm 0.122$ & $0.027 \pm 0.008$ & $0.000 \pm 0.000$ & $0.135 \pm 0.040$ & $0.000 \pm 0.040$
\end{tabular}

1'Locations are designed with a three-letter code: ESC, El Escorial; PAL: Palazuelos; POL, Pollos; SCR, Santa Cruz de Retamar.

$\mathrm{N}$ : number of sequences; $\mathrm{H}$ : number of haplotypes; $\mathrm{H}_{\mathrm{d}}$ : haplotype diversity; $\pi$ : genetic diversity; SE: standard error. 


\subsubsection{Analysis of ecological factors affecting WHMV population genetic diversity}

The ecological factors affecting WHMV population $\mathrm{H}_{\mathrm{d}}, \pi, \mathrm{d}_{\mathrm{N}}, d_{S}$ and $d_{N} / d_{S}$ were analysed using multiple regression model selection analyses (Table 4.37). The same variables as for predicting potyvirus species-specific prevalence and adding WHMV prevalence as an additional predictor (data of predictor variables is in Section 4.3, Tables 4.27 and 4.28) were utilized. The best-ranked model explaining $\mathrm{H}_{\mathrm{d}}$ contained plant relative abundance and rainfalls as the most important predictors $(r=0.78 ; P=0.007)$. In this model plant relative abundance had similar relative importance than rainfalls (30\% and $26 \%$, respectively) (Table 4.37). Bivariate analysis showed a negative relationship between plant relative abundance and $H_{d}(r=-0.25 ; P=0.042)$, whereas the association with rainfalls was positive but not significant $(r=0.14 ; P=0.142)$ (Figure 4.31A,B). The best-ranked models predicting WHMV genetic diversity $(\pi)$ contained rainfalls and virus prevalence as the most important predictors $(r=0.71$; $P=0.003$ ), rainfalls having higher relative importance than virus prevalence (50\% and $27 \%$, respectively). Bivariate analysis showed a positive association between rainfalls and $\pi(r=0.29 ; P=0.025)$, whereas the association with WHMV prevalence was negative ( $r=-0.25 ; P=0.042)$ (Figure 4.31C,D). The best-ranked models predicting WHMV population $d_{N}$ and $d_{N} d_{S}$ contained plant biomass and WHMV prevalence as the most important predictors, plant biomass having the highest relative importance (46\%) ( $r>0.78 ; P=0.002)$ (Table 4.37). Bivariate analyses indicated a positive association between plant biomass and the studied parameters $(r>0.32 ; P<0.017)$ (Figure 4.31E,I), whereas the association with virus prevalence was negative and only marginally significant $(r<-0.21$; $P>0.062$ ) (Figure 4.31F, J). The best-ranked model predicting $d_{s}$ contained plant biomass and rainfalls as the most important predictors $(r=0.81 ; P=0.012)$, the former predictor having the highest relative importance (46\%) (Table 4.37). Bivariate analyses showed a positive association between these two predictors and the studied parameters, although the association was only significant with plant biomass ( $r=0.44 ; P=0.004)$ (Figure 4.31G,H). 


\section{No other combination of factors yielded a model that closely competed with the best-ranked one (Appendix 6, Table A6.1).}

Table 4.37. Model selection analyses for WHMV population haplotype diversity $\left(H_{d}\right)$, genetic diversity $(\pi)$, number of synonymous $\left(d_{S}\right)$ and non-synonymous $\left(d_{N}\right)$ substitutions per site, and selection pressures $\left(d_{N} / d_{S}\right)$. Model structures included host plant density, host relative abundance, plant species richness and plant biomass; and temperature, relative humidity and rainfall in the sampled locations (minimal, maximal and average values) as predictors, and season as covariate. WHMV prevalence was also included as predictor of virus evolution parameters. Best-ranked models are shown.

\begin{tabular}{|c|c|c|c|c|c|}
\hline Model structure & $r^{\dagger}$ & logLik & $\mathrm{AlC}^{\ddagger}$ & $\Delta_{\mathrm{i}} \S$ & $\boldsymbol{\omega}_{i}^{\mathbb{T}}$ \\
\hline \multicolumn{6}{|l|}{$H_{d}$} \\
\hline $\mathrm{PRA}(30)+\mathrm{RAF}(26)+\mathrm{PRE}(12)+\mathrm{R} \mathrm{H}_{\text {mean }}(12)+\mathrm{T}_{\text {mean }}(11)+\mathrm{V}(9)$ & $0.78^{*}$ & 37.97 & -59.94 & 0.00 & 0.55 \\
\hline $\mathrm{PRA}(29)+\mathrm{RAF}(26)+\mathrm{PRE}(12)+\mathrm{RH}_{\text {mean }}(12)+\mathrm{T}_{\text {mean }}(11)+\mathrm{V}(8)+\mathrm{S}(2)$ & $0.79^{*}$ & 38.15 & -58.30 & 1.64 & 0.24 \\
\hline $\mathrm{PRA}(30)+\mathrm{RAF}(26)+\mathrm{RH}_{\text {mean }}(12)+\mathrm{PRE}(11)+\mathrm{T}_{\text {mean }}(11)+\mathrm{V}(8)+\mathrm{HPD}(2)$ & $0.78^{*}$ & 37.97 & -57.94 & 2.00 & 0.20 \\
\hline \multicolumn{6}{|l|}{$\pi$} \\
\hline $\mathrm{RAF}(50)+\mathrm{PRE}(27)+\mathrm{RH}_{\text {mean }}(17)+\mathrm{T}_{\text {mean }}(6)$ & $0.71^{*}$ & 66.22 & -120.44 & 0.00 & 0.25 \\
\hline $\mathrm{RAF}(50)+\mathrm{PRE}(28)+\mathrm{RH}_{\text {mean }}(22)$ & $0.67^{*}$ & 65.16 & -120.33 & 0.11 & 0.24 \\
\hline $\mathrm{RAF}(46)+\mathrm{PRE}(28)+\mathrm{RH}_{\text {mean }}(16)+\mathrm{PRA}(4)+\mathrm{T}_{\text {mean }}(6)$ & $0.71^{*}$ & 66.47 & -118.94 & 1.50 & 0.12 \\
\hline \multicolumn{6}{|l|}{$d_{N}$} \\
\hline$V(46)+P R E(21)+R A F(17)+R H_{\text {mean }}(9)+T_{\text {mean }}(7)$ & $0.78^{*}$ & 49.73 & -85.46 & 0.00 & 0.38 \\
\hline$V(41)+P R E(22)+R A F(17)+R H_{\text {mean }}(10)+T_{\text {mean }}(7)+S(3)$ & $0.80^{*}$ & 50.49 & -84.99 & 0.47 & 0.30 \\
\hline$V(45)+P R E(19)+R A F(16)+R H_{\text {mean }}(10)+T_{\text {mean }}(7)+P R A(3)$ & $0.79^{*}$ & 49.97 & -83.94 & 1.52 & 0.18 \\
\hline \multicolumn{6}{|l|}{$d s$} \\
\hline $\mathrm{V}(46)+\mathrm{RAF}(13)+\mathrm{PRE}(12)+\mathrm{RH}_{\text {mean }}(9)+\mathrm{T}_{\text {mean }}(8)+\mathrm{S}(8)+\mathrm{PRA}(4)$ & $0.81^{*}$ & 43.62 & -69.25 & 0.00 & 0.46 \\
\hline $\mathrm{V}(57)+\mathrm{RAF}(11)+\mathrm{PRE}(10)+\mathrm{RH}_{\text {mean }}(9)+\mathrm{T}_{\text {mean }}(8)+\mathrm{PRA}(5)$ & $0.77^{*}$ & 42.35 & -68.70 & 0.55 & 0.35 \\
\hline $\mathrm{V}(44)+\mathrm{RAF}(13)+\mathrm{PRE}(10)+\mathrm{RH}_{\text {mean }}(9)+\mathrm{T}_{\text {mean }}(8)+\mathrm{S}(7)+\mathrm{HPD}(5)+\mathrm{PRA}(4)$ & $0.81^{*}$ & 43.79 & -67.57 & 1.67 & 0.20 \\
\hline \multicolumn{6}{|l|}{$d_{N} / d_{s}$} \\
\hline $\mathrm{V}(45)+\mathrm{PRE}(23)+\mathrm{RAF}(16)+\mathrm{RH} \mathrm{H}_{\text {mean }}(9)+\mathrm{T}_{\text {mean }}(7)$ & $0.79^{*}$ & 25.37 & -36.74 & 0.00 & 0.41 \\
\hline $\mathrm{V}(41)+\mathrm{PRE}(23)+\mathrm{RAF}(16)+\mathrm{RH}_{\text {mean }}(9)+\mathrm{T}_{\text {mean }}(8)+\mathrm{S}(3)$ & $0.80^{*}$ & 25.98 & -35.96 & 0.78 & 0.28 \\
\hline $\mathrm{V}(43)+\mathrm{PRE}(19)+\mathrm{RAF}(16)+\mathrm{RH}_{\text {mean }}(10)+\mathrm{T}_{\text {mean }}(7)+\mathrm{PRA}(5)$ & $0.79^{*}$ & 25.43 & -34.85 & 1.89 & 0.16 \\
\hline
\end{tabular}

*The relative importance (\%) of each predictor variable is shown in parenthesis.

tCorrelation coefficient. Asterisks indicate significant correlations $(P<0.05)$.

$\ddagger$ Akaike’s Information Criterion.

$\S_{\Delta}$, is the difference between the AIC of a given model and that of the best-ranked model, and quantifies how models compete (best-ranked model: $\Delta_{i}=0$; substantial empirical support: $\Delta_{i}=1-2$; considerable less support: $\Delta_{i}=2-7$; and no support; $\Delta>10$ ) (Burnham and Anderson, 2002).

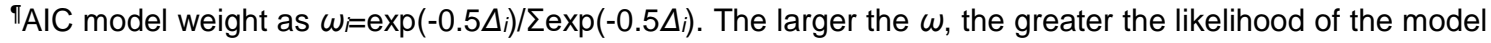
relatively to the competing models. Maximum $\omega,=1$.

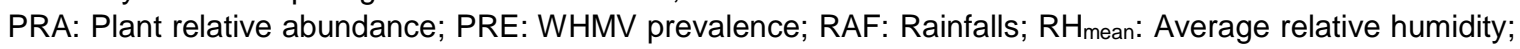
$\mathrm{T}_{\text {mean }}$ : Average temperature; V: Plant biomass; HPD: Host plant density; S: Species richness. 
A

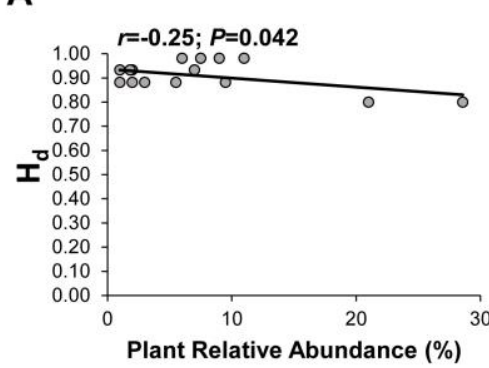

C

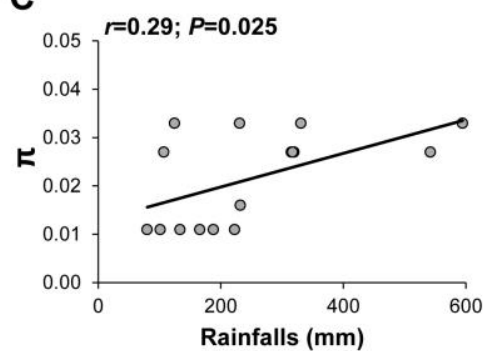

\section{E}

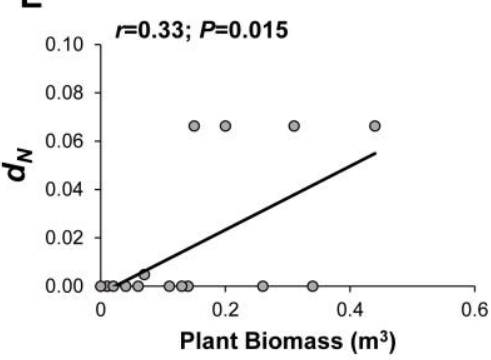

G

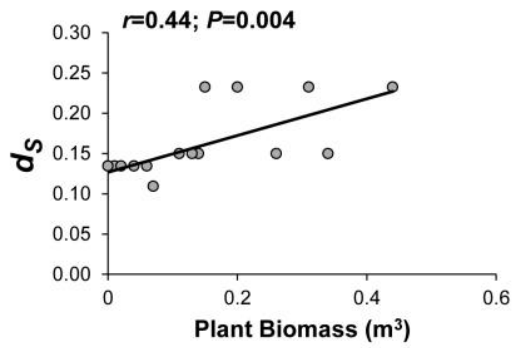

I

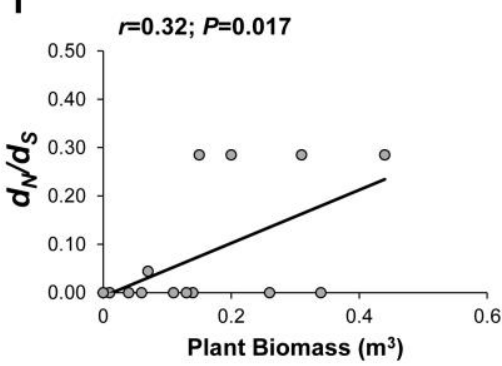

B

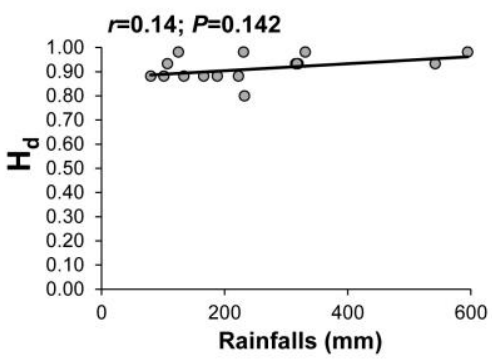

D

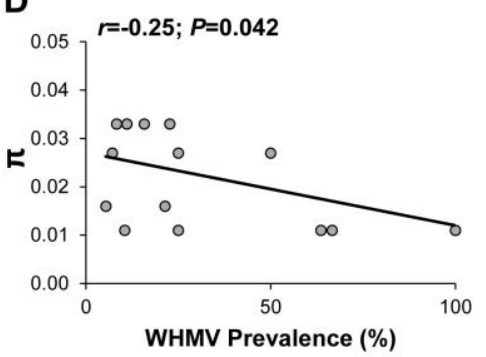

$\mathbf{F}$

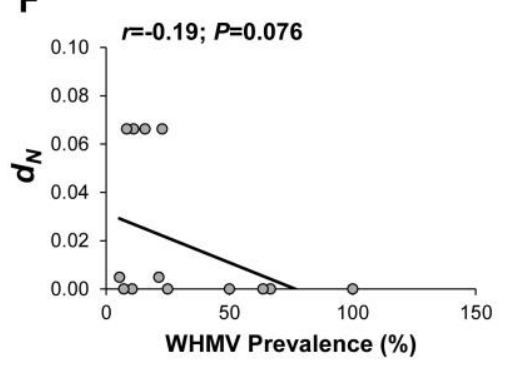

H

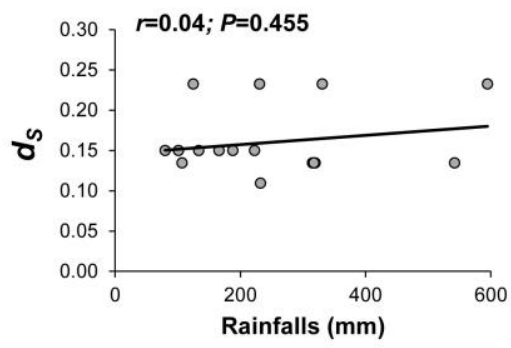

J

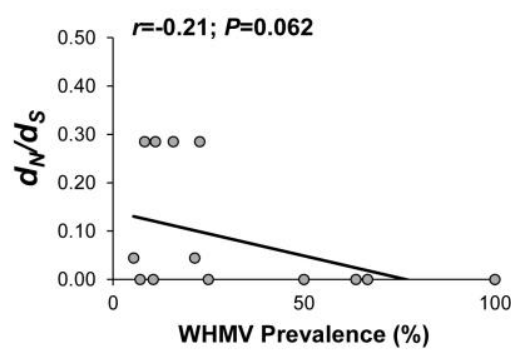

Figure 4.31. Bivariate relationships between WHMV population evolutionary parameters and the best predictors. The associations between potyvirus population haplotype diversity (A and $B)$, population genetic diversity ( $C$ and $D)$, number of non-synonymous ( $E$ and $F$ ) and synonymous mutations per site $(G$ and $H)$ and overall selection pressures $d_{N} / d_{s}(I$ and $J)$ and best predictors are represented. 


\subsubsection{Analysis of WHMV speciation mode}

Both Al and PS statistics revealed a significant association between the WHMV phylogeny and the location where the sequences were collected $\left(P<1 \times 10^{-5}\right)$. MC statistic indicated that this result was due to the stronger clustering of the sequences collected in El Escorial, Pollos and Santa Cruz de Retamar $(P<0.01)$ (Figure 4.32).

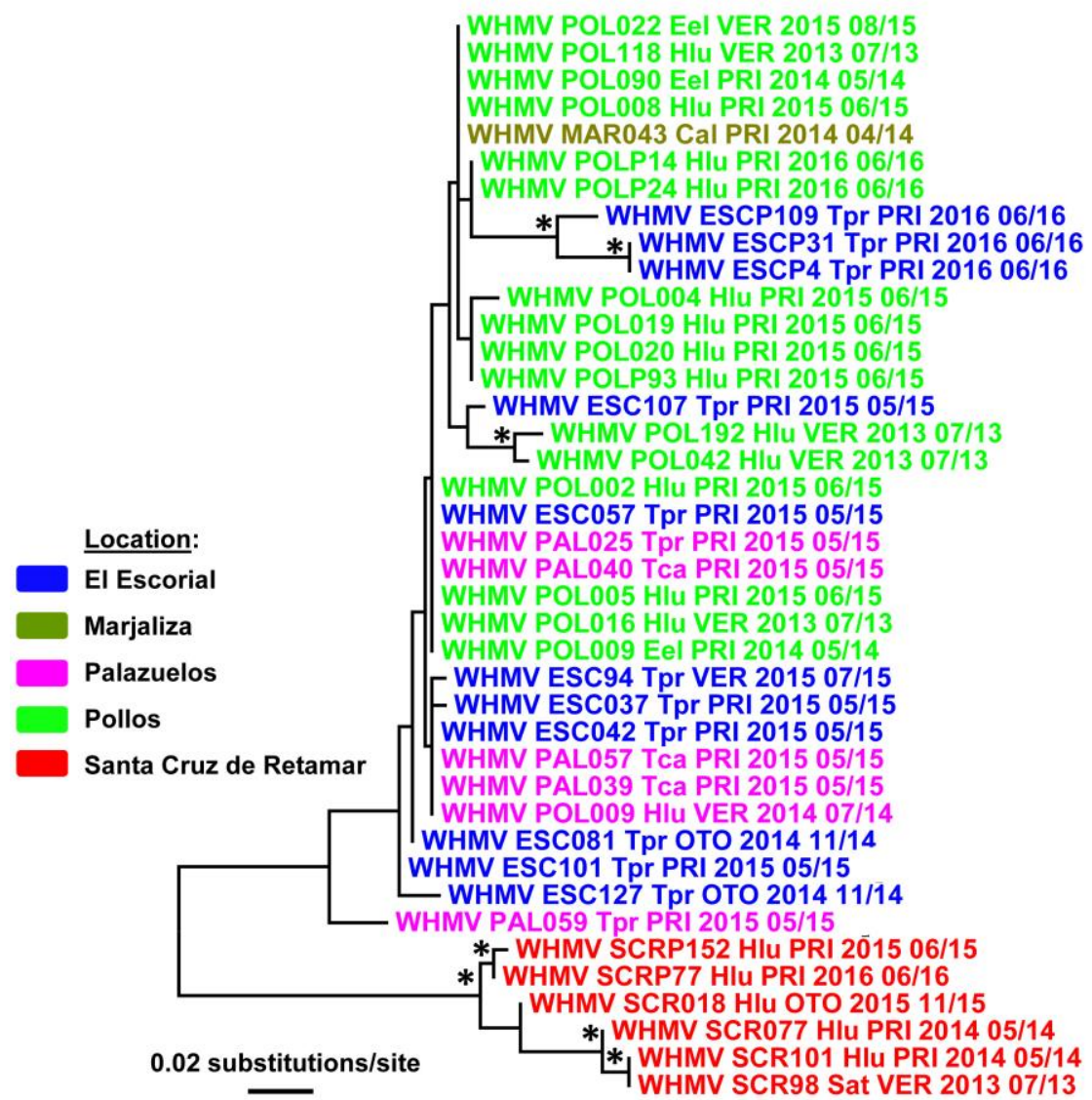

Figure 4.32. Maximum-likelihood phylogeny based on the Nlb region representing the association between WHMV phylogeny and geographical location. Asterisks indicates nodes with a bootstrap support $\geq 0.90$.

In addition, Al and PS statistics showed a significant genetic structure of the WHMV populations according to host species $\left(P<1 \times 10^{-5}\right)$. In this case, according to the MC statistics, the significance was due to the stronger clustering of the virus sequences collected from Trifolium pratense and Humulus lupulus $(P<0.01)$ (Figure 4.33). 


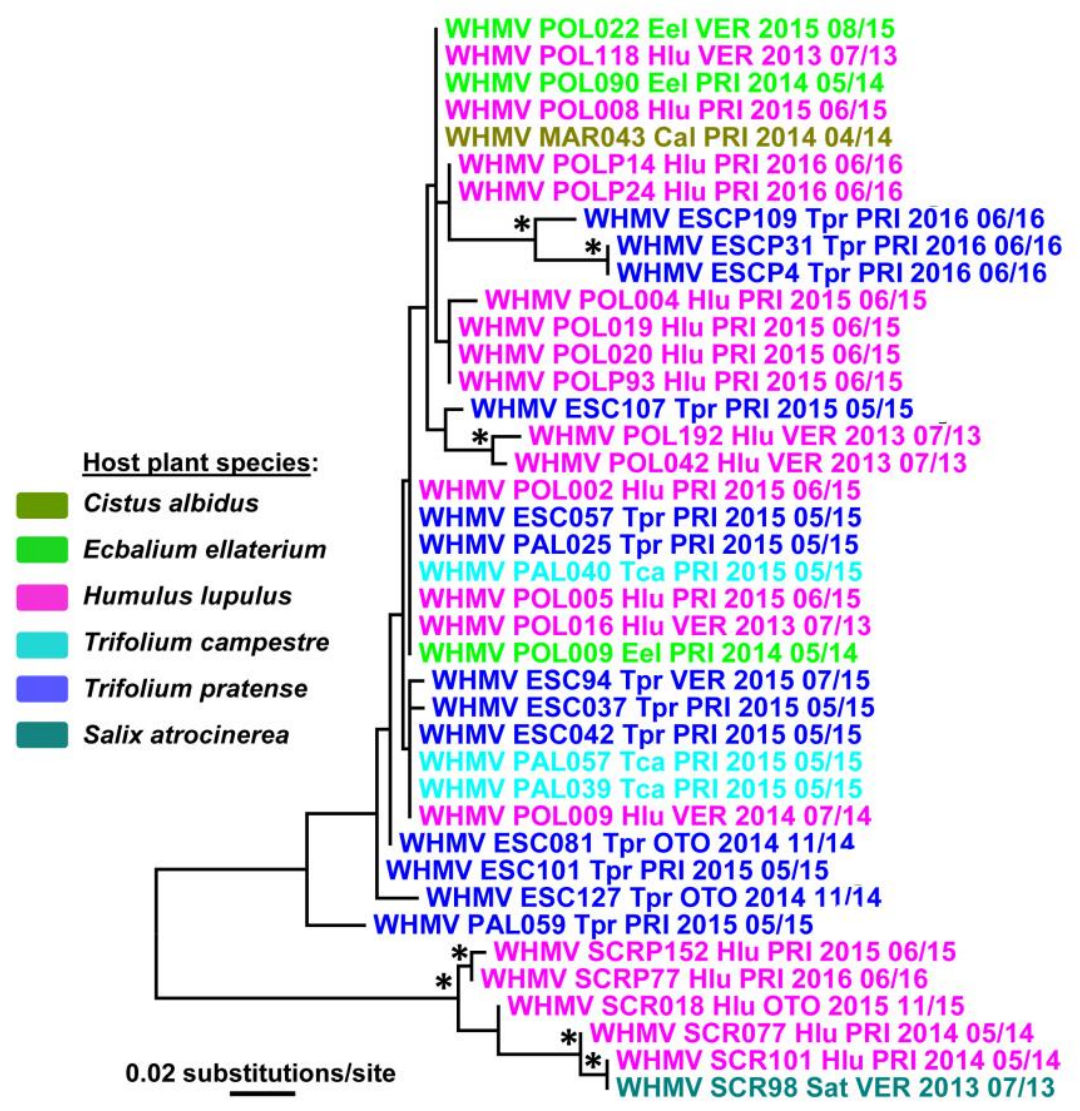

Figure 4.33. Maximum-likelihood phylogeny based on the Nlb region representing the association between WHMV phylogeny and host plant species. Asterisks indicates nodes with a bootstrap support $\geq 0.90$.

These data suggest that WHMV speciation tends to occur within the same host species and in the same geographic area, which is compatible with genetic diversification through sympatric speciation, at least for certain hosts. 
5. DISCUSSION 
Most of our knowledge on plant-virus interactions comes from the study of viruses that cause diseases in crops. Therefore, our understanding of plant viruses might be biased towards those interactions that have the most obvious agro-economic consequences. Indeed, until recently weedy and wild plants were considered of interest only as source/reservoir of virus inoculum for crops (Cooper and Jones, 2006; Jones, 2009; Alexander et al., 2014). The advent of next generation sequencing (NGS) has allowed exploration of the virome of a number of different ecosystems (e.g., Delwart, 2007; Hurwitz and Sullivan, 2013), including wild plant communities (Roossinck, 2012; Stobbe and Roossinck, 2014). These studies have shown that plant-virus interactions in wild ecosystems are far more diverse than in agricultural ecosystems: in wild plants virus infections are common, and virus diversity and identity appear to be different from that in crops (Roossinck, 2012; Stobbe and Roossinck, 2014). Indeed, NGS analyses in wild plants have uncovered large (previously) unknown virus diversity (Roossinck, 2011). These observations suggest that plant viruses are important ecological agents in wild plant communities. Thus, the detailed genomic and biological characterization of viral diversity in wild ecosystems, and the analysis of their population dynamics is central to fully understand plant-virus interactions, and will yield information on the processes that lead to disease emergence in these ecosystems (Pagán et al., 2016). However, most of the virus species present in wild ecosystems remain uncharacterized (Roossinck, 2012; Stobbe and Roossinck, 2014), and the determinants of their epidemiology and evolution are largely unknown (Pagán et al., 2016).

To address these questions, this thesis analyses the ecological determinants of plant virus prevalence and population genetic diversity in two wild ecosystems, which accounts for the largest area of wild ecosystems in the Iberian Peninsula: evergreen oak and riparian forests. The thesis uses the species of the genus Potyvirus as a model, as this genus is the largest of plant viruses and various species within the genus have been reported to infect plant species commonly found in the ecosystems studied. Through surveys done between 2013 and 2016, and using state-of-the-art bioinformatics approaches, the present work provides information on the prevalence, host range and population genetic diversity of the potyvirus species detected in evergreen oak 
and riparian forests. At different taxonomical scales (genus vs. species) and considering different virus life-history strategies (specialist vs. generalist) this thesis identifies the ecological determinants affecting the prevalence and population genetic diversity of potyviruses.

\subsection{Characterization of evergreen oak forest and riparian forests in the Iberian Peninsula}

For this thesis, the locations of evergreen oak and riparian forests selected were chosen such that there was no evidence of human intervention and could be considered as wild ecosystems. However, large areas of the Iberian Peninsula, and often those adjacent to the monitored locations, have been under human management for long periods of time. Thus, it could be possible that the floristic composition of the forest locations utilized in this thesis had been indirectly influenced by the changes in the vegetation of the adjacent human-managed fields. For instance, plant species predominant in the nearby managed fields may have invaded the evergreen oak and riparian forests, altering the typical flora of these forests. Because human management generally leads to a homogenization of the flora, it could also be possible that its indirect influence in wild ecosystems may result in the reduction of the differences in biodiversity and floristic composition between the two analysed ecosystems that has been generally reported (Blanco et al., 2005; Loidi, 2017). However, the analyses of the floristic composition in the monitored locations (section 4.1) indicated that it differed between both ecosystems, and the typical species that characterize the plant communities of riparian and evergreen oak forests in Central Spain were present (Blanco et al., 2005; Loidi, 2017). Differences in plant community composition between both ecosystems were also accompanied by different proportion of annual and perennial plant species. Riparian forests supported a predominance of perennials, while annual plants accounted for $40-50 \%$ of the species present in evergreen oak forests. The highest percentage of annuals in the later ecosystem agrees with previous observations in Mediterranean evergreen oak forests, as annuals are best adapted to the seasonal regime of mild moist winters and dry summers that 
characterize the climate where these forests predominate (Archibold, 1995; Bruno et al., 2014). In addition, biodiversity was significantly higher in riparian forests than in evergreen oak forests, as expected (Blanco et al., 2005; Loidi, 2017). This higher plant biodiversity was associated with higher biomass in riparian forests than in evergreen oak forests, as is characteristic of these types of ecosystems (Blanco et al., 2005). Therefore, the locations selected for this study are representative of the two ecosystems under study. This conclusion is also supported by the analyses of sampling effort, which indicate that the sampling scheme used here captured most, and in many cases all, the species diversity in the monitored locations (see Figure 4.2 and 4.3).

At odds with differences in biodiversity, floristic composition and plant biomass, climatic factors (temperature, relative humidity and rainfalls) did not generally vary between evergreen oak and riparian forests. Previous works have reported differences in climatic conditions between evergreen oak and riparian forests (Blanco et al., 2005; Loidi, 2017). There are two possible explanations for this contradiction that are not mutually exclusive: (i) because the evergreen oak and riparian forests selected here are both in an area of Mediterranean climate, climatic conditions are similar. Note that the Mediterranean climate is characterized by large variations in the climatic conditions within the same season, which results in large standard errors of average climatic parameters that may mask differences between seasons/ecosystems. (ii) Because the stations providing climatic parameters were not exactly in the monitored locations, the data may have not fully capture the microclimatic conditions of riparian forests that extend near the river streams (Naiman and Decamps, 1997). In this case, rainfalls would be the less affected parameter, as stations were close enough to the locations and at a similar altitude to have a sufficiently good measure of this parameter. Indeed, rainfalls was the climatic parameter in which differences between ecosystems were more obvious (see Table 4.4). Likely, temperature and relative humidity in riparian forest locations were more poorly capture by the nearby climatic stations. Indeed, other relative humidity measures recorded in situ, as was the case of soil moisture, showed higher values in riparian that in evergreen oak forest. Interestingly, soil moisture has been shown to be a meaningful indicator 
of the climatic conditions in riparian forests (Rodà, 1999), and may compensate for the lack of precision in other climatic variables.

\subsection{Ecological determinants of genus-wide potyvirus infection risk in wild ecosystems}

In this thesis, potyvirus prevalence was used as a proxy of infection risk. Potyvirus infection was detected at every season and sampling year in evergreen oak and riparian forests, indicating that potyvirus infection occur consistently in both ecosystems. Potyvirus prevalence fluctuated between seasons and years, but on average infection prevalence was of $3.80 \%$ in riparian forests and of $2.09 \%$ in evergreen oak forests. This prevalence is smaller than that reported in other wild plant populations, which indicated that an average of $10 \%$ of individuals in a population are infected (reviewed by Prendeville et al., 2012). However, note that this estimate refers to plants infected by any virus, and this thesis focused in a specific virus genus. The few reports of species-specific prevalence in wild plant populations have often report values closer to those reported here (e.g. Sacristán et al., 2004; Cooper and Jones, 2006; Tugume et al., 2008; McLeish et al., 2017).

Potyvirus prevalence was generally higher in riparian than in evergreen oak forests, which was associated with higher plant species richness and abundance of host plants in the former ecosystem. Interestingly, this result would support the "Amplification Effect" hypothesis: as the number of total species in the ecosystem increases, the number of host species increases leading to increased pathogen prevalence, and therefore infection risk (Keesing et al., 2006; Ostfeld and Keesing, 2012). This is in disagreement with accumulating evidence in a variety of animal-pathogen (LoGiudice et al., 2003; Keesing et al., 2010; Roche and Guégan 2011) and plant-pathogen (Mitchell et al., 2003; Hass et al., 2011; Pagán et al., 2012; Civitello et al., 2015) systems, which support the "Dilution Effect" hypothesis that states that higher species diversity decreases the abundance of hosts, resulting in a reduction in the number of contacts between susceptible and infected individuals and therefore 
in lower pathogen prevalence (Keesing et al., 2006; Ostfeld and Keesing, 2012).

The conditions in which dilution and amplification effects are more likely to occur, and the spatial scale at which such effects manifest, are still under debate (Randolph and Dobson, 2012; Johnson et al., 2013; Johnson et al., 2015). For instance, as the relationship between biodiversity and pathogen prevalence will often depend strongly on spatial scale, different effects could be expected at the local analysis or at the community scale (Johnson et al., 2015). Indeed, recent work has shown that virus prevalence in plant populations of different habitats conforms the "Dilution" or the "Amplification Effects" depending on the spatial scale of the analysis. In these plant populations, the prevalence-diversity relationship was habitat specific, showing both amplification and dilution effects at the habitat spatial scale, whereas only an amplification effect was detected at the larger ecosystem spatial scale (McLeish et al., 2017). Similarly, the analyses of this thesis, which are done at the ecosystem spatial scale, also agree with the "Amplification Effect" hypothesis.

In addition, the prevalence-diversity relationship may be driven by the identity of the particular species present in the ecosystems/communities than diversity per se (Randolph and Dobson, 2012). On this regard, the life cycle of the plant species present in an ecosystem could be a relevant trait to understand how biodiversity determines pathogen prevalence (Johnson et al., 2012). Theoretical work has proposed that annual hosts are better pathogen reservoirs than perennials ones: perennial species are more likely to be challenged against pathogen infections during their life span, and therefore invest in developing defense mechanisms. Conversely, short-lived hosts will invest less in costly defenses against pathogens, so that they will be more susceptible to infection and/or will sustain larger vector populations, thus being effective reservoirs for infectious diseases (Miller et al., 2007; Bruns et al., 2015). At odds, the percentage of perennial hosts was much larger in riparian than in evergreen oak. Moreover, in riparian forests there was a higher percentage of perennial hosts than of total plant perennial species, indicating a preference of potyviruses for infecting plants with longer life cycles. However, the above-mentioned theoretical models considered resistance as the single major defence mechanisms. Mathematical models considering both resistance 
and tolerance predicted that higher abundance of perennial hosts would be associated with increased pathogen prevalence: perennial hosts would be more likely to evolve tolerance, rather than resistance, which would increase pathogen spread rates and prevalence by serving as long-term sources of infection for susceptible individuals (Herms and Mattson, 1992; Boots and Bowers, 2004; Borer et al., 2010). Our results would be compatible with this prediction, and are also in agreement with studies showing that virus prevalence increases with higher abundance of perennial hosts (Borer et al., 2010; Malmstrom et al., 2005b; Seabloom et al., 2013, but see Alexander et al., 2017). Thus, the results of this thesis show the importance of considering not only the number of species/relative abundance, but also their identity, to understand the infection risk, and consequently the emergence, of virus epidemics (Randolph and Dobson, 2012; Keesing et al, 2006; Johnson et al., 2015).

Potyvirus prevalence in riparian and evergreen oak forests, two ecosystems that greatly differ in their ecology, appears to be determined by the species richness and identity in terms of their life cycle. However, are these factors also important determinants of virus prevalence over time within the same ecosystems, or are there other relevant ecological/climatic factors? In order to answer this question, the association between the ecological factors that characterize evergreen oak and riparian forests locations and the ecosystem-specific potyvirus prevalence was analysed using Machine Learning (ML) based-methods. Complete ML models including all predictors of potyvirus prevalence considered in this thesis performed better than the baseline model and generally showed high predictive power regardless of the method used (Tables 4.10 and 4.11). The three utilized methods identified the identity of the plant species present in the ecosystem as the major (and often the solely) determinant of potyvirus prevalence in both evergreen oak and riparian forests. The presence/absence of host species such as $H$. lupulus, T. pratense or $R$. montana (many of the perennial) defined the different levels of potyvirus prevalence, whereas the presence/absence of non-host species defined the scenarios of no virus presence. As stated above, early theory on the relationship between biodiversity and infection risk predicted that species richness would be the more relevant factor in defining this relationship (Keesing 
et al., 2006; Ostfeld and Keesing, 2012). More recent elaborations incorporated the identity of the species in the ecosystem as a key factor in determining infection risk: if higher species richness results in higher number of host species infection risk would be higher, and if higher species richness is due to a larger number of non-host species infection risk would decrease (Randolph and Dobson, 2012; Johnson et al., 2015). The results of this thesis support the key role of species identity even over species richness in determining infection risk, and are also in agreement with previous works indicating that plant virus prevalence is associated with the presence of certain species in the ecosystem, and not only with a general increment in the ecosystem species richness (Malpica et al., 2006; McLeish et al., 2017).

Because the overly high importance of plant species as determinant of virus infection risk could mask the effect of other factors, ML models excluding plant species identity traits as predictor were constructed. Although these models differed in the factors identified as the most important predictors of potyvirus prevalence and in the relative importance of these predictors, some general patterns arise. All ML models identified plant relative abundance as a major predictor of potyvirus prevalence in the two studied ecosystems, and bivariate relationships between potyvirus prevalence and host relative abundance indicated a (weak) negative association between these traits. This result would be in agreement with theory attributing to host relative abundance a key role in infection risk and/or pathogen transmission (Keesing et al., 2006; Ostfeld and Keesing, 2012). However, this theory predicts a positive association between host relative abundance and infection risk, whereas the opposite relationship was observed in riparian and evergreen oak forests (Figure 4.18). This difference with theoretical predictions would be explained if: (i) the best potyvirus hosts are those that appear less frequently in the studied ecosystems. This seems to be the case for host species of other plant viruses such as CMV in several habitats in the Iberian Peninsula (McLeish et al., 2017). (ii) Virus infection increases host mortality such that higher virus prevalence results in larger decrease of the host population size (and therefore of host relative abundance). Although metagenomics analyses in wild ecosystems have shown that virus infection generally does not result in the expression of disease symptoms (Prendeville et al., 2012; Roossinck, 2012), some examples exist of 
plant viruses that cause obvious diseases, affect plant population sizes and plant ecosystem composition (Power and Mitchell, 2004; Malmstrom et al., 2005a; Power et al., 2011; Rua et al., 2011; Rodelo-Urrego et al., 2013; Prendeville et al., 2014). This could be the case of potyviruses infecting the ecosystems studied in this thesis, especially for evergreen oak forests (see section $\mathbf{5 . 3}$ for further discussion). (iii) Plant isolation favours infection. Indeed, several studies have reported a negative correlation between plant density and virus prevalence, which was attributed to migratory aphids landing preferentially on isolated large plants (A'Brook 1964; Way and Heathcote, 1966).

The second factor generally detected by ML models as predictor of potyvirus infection risk was host density, with bivariate analyses shown no (evergreen oak forests) or weak (riparian forests) positive association between these traits. The importance of this factor in the ML models constructed in this thesis supports the generally assumed idea that higher plant density increases infection risk (Burdon and Thrall, 2008). The aggregated distribution of host plants within each location may favor virus transmission by aphids, and consequently may increase infection risk. This is also in agreement with previous work in other plant virus-wild host interactions that also identified host density as a key factor for plant virus infection risk (Malmstrom et al., 2005a; Borer et al., 2010; Pagán et al., 2012; Rodelo-Urrego et al., 2013). Finally, the third predictor commonly detected by all ML methods was plant life cycle, with the presence of perennial plants favouring higher potyvirus prevalence. This would be in agreement with results discussed in section 5.1. Also, the importance of perennial plants as potyvirus hosts fits with other of the results discussed in this section. For instance, perennial hosts tend have less relative abundance than annual hosts in the analysed ecosystems, especially given that they have larger adult sizes (our observations). This would be compatible with the negative relationship between potyvirus prevalence and host relative abundance. Also, perennial hosts usually form aggregated clusters, which would explain at least in part the positive association between host density and potyvirus prevalence.

It should be noted that ML models that excluded plant species identity traits did not accurately predicted potyvirus infection risk in any ecosystem, showing performance scores close or even lower than the baseline model in 
both training and validation sets (Tables 4.13 and 4.14). The comparison of ML models predicting potyvirus presence/absence and potyvirus intensity of infection yielded an explanation for this result. In ML models predicting potyvirus presence/absence, including only plant virus identity as predictor was enough to accurately predict potyvirus presence. On the other hand, for models predicting virus intensity the most relevant factors were host relative abundance and density. Thus, the identity of the species present in an ecosystem defines the chances for a virus to enter in such ecosystems, with other ecological factors poorly predicting non-host of uninfected prevalence categories. Once the virus entered the ecosystems (i.e., only categories of virus prevalence are considered), the capacity of the virus to invade the ecosystem depends mostly on other ecological factors. Interestingly, this patterns hold for two ecosystems with very different ecological characteristics, which argues on favour of the generality of the observed patterns.

\subsection{Ecological determinants of species-specific potyvirus infection risk in wild ecosystems}

Ecological factors that affect genus-wide Potyvirus prevalence in evergreen oak and riparian forests yield relevant information about virus epidemiology in the monitored ecosystems. Nevertheless, for certain virus species some ecological factors may be more important than others, according to the specific characteristics of such virus species (Jones, 2009). Previous works highlighted the importance of knowing the host range of the pathogen(s) under study to understand how host and virus ecology affect virus prevalence and hence, infection risk (Malmstrom et al., 2005a; Borer et al., 2010; Pagán et al., 2012; McLeish et al., 2017). However, it is rare to find in the literature an analysis of this subject in which the pathogen host range is fully characterized in a given ecosystem (but see McLeish et al., 2017). In this thesis the importance of ecological factors in determining Potyvirus species-specific prevalence according to the virus host range was analysed.

Identification of the potyviruses present in wild plants of both ecosystems indicated the presence of novel species of genus Potyvirus. Discovery of novel 
species is common in virus surveys using metagenomics methods, but because in this type of studies the samples often represent pools of many hosts, little or no connection can be made to the virus host(s) (Roossinck and García-Arenal, 2015; Roossinck, 2015). The methodology used in this thesis allowed to fully characterize the natural host range of the known and novel potyvirus species present in the monitored ecosystems. Four viral species were identified in this thesis, of which CIYVV and ENMV, are known potyvirus species that have been commonly found infecting cultivated species. ENMV is present in the Mediterranean basin in a large set of wild and cultivated plants, mainly of the family Asteraceae (Desbiez et al., 2017). In this thesis, sequences related to this potyvirus species were identified infecting a non-previously known host species in the family Asteraceae. Phylogenetic analyses of all available ENMV Nlb nucleotide sequences hosts separated isolates from wild hosts and from cultivated ones. Failure in amplifying the CP gene of the ENMV isolates from wild hosts prevented confirming this observation. However, results suggest that ENMV isolates of wild and cultivated hosts are genetically different. CIYVV is one of the most prevalent potyviruses in legumes, causing important economic losses in crops and pastures worldwide (Andrade et al., 2007). In riparian forests of the Iberian Peninsula, CIYVV has been detected in legumes (Trifolium species) and host species of other families (Polygonaceae), which has not been reported as CIYVV host to date. Both $\mathrm{Nlb}$ and $\mathrm{CP}$ sequences of CIYVV identified in this study clustered in the same monophyletic group than available sequences of this virus. The analysis of this thesis also identified two new potyvirus species (MeRV and WHMV). Host range of MeRV was limited to the perennial plant Ruta montana in a location of evergreen oak forests, being highly prevalent in this host plant. Finally, WHMV had a broader host range and geographic distribution, infecting mostly perennial plant species from different botanical families collected in locations of riparian forests (Cannabaceae, Cucurbitaceae, Fabaceae and Salicaceae) and evergreen oak forests (Cistaceae). Hence, this study significantly extended the knowledge of natural hosts of known viruses of the genus Potyvirus in wild ecosystems.

In the analyzed ecosystems, MeRV and EMNV behaved as specialists, because they infected a single host species; whereas WHMV and CIYVV were detected infecting plant species from different botanical families thus, behaved 
as generalists. In order to study how the ecological factors recorded in evergreen oak and riparian forests could differentially influence virus prevalence according to virus host range, this thesis focused in the most prevalent virus representative of each strategy: MeRV as a specialist virus, and WHMV as a generalist virus.

MeRV would represent one of the first examples of specialist plant viruses native of wild ecosystems, as most plant viruses described in wild perennial plants naturally infect broader host ranges (Cooper and Jones, 2006). Specialist viruses are often extremely well adapted to their host(s). This means that these pathogens are highly transmissible and efficiently exploit host resources to maximize its fitness (Woolhouse et al., 2001; García-Arenal and Fraile, 2013). Theoretical models of host adaptation assume that pathogen transmission is positively correlated with its multiplication and negatively correlated with its virulence. In turn, multiplication is positively associated with virulence, which lead to a trade-off between transmission and virulence. Thus, these models predict that pathogen fitness would be optimal at intermediate levels of virulence (Anderson and May, 1982). Despite behaving as specialist, MeRV infection did not induced any apparent symptom in mountain rue, neither in the field nor in the greenhouse. Similarly, infections by any of the other potyviruses detected in evergreen oak forests, which also showed narrow host ranges, were also asymptomatic. This would be in agreement with previous studies that generally reported a lack of obvious symptoms associated with virus infections in wild plants (Cooper and Jones, 2006; Roossinck, 2010; Prendeville et al., 2012). The apparent absence of symptoms in MeRV-infected mountain rue plants could be explained if: (i) MeRV is not optimally adapted to mountain rue, which is unlikely given the high prevalence of the virus; (ii) adaptation leads to reduced virulence; and (iii) negative effects of virus infection are not associated with plant growth but with other host traits. Some of our results support the later possibility. Given that infected plants cannot clear infection, and that mountain rue is a perennial plant, we would expect relatively homogeneous virus prevalence across seasons if virus adaptation would lead to reduced virulence (Fraile et al., 2017). However, MeRV prevalence variable even within the same sampling cycle. This suggests that MeRV infection could have an effect on plant survival. Optimal temperature for seed germination of 
species from the Ruta genus is about $30^{\circ} \mathrm{C}$ (Mguis et al., 2011), and consequently young plants are more abundant in summer. Given that maximum MeRV prevalence occurs in this season and decays afterward, it could be hypothesized that MeRV-induced mortality could be more frequent in young individuals. In support of this hypothesis, additional surveys of mountain rue plants in evergreen oak forests indicated that MeRV was less prevalent in older plants (i.e., more than a year-old and at least one flowering period) $(40 \%, 6 / 15$ plants) than in younger plants (i.e., less than a year- old and not flowered) (71\%, 5/7 plants). This indicates that not every young infected individual reaches the adult stage. Hence, our data strongly suggests that MeRV is an important modulator of the population dynamics of its host. On the other hand, this does not seem to be the case in crop hosts, as we failed in detecting the virus in pepper and tomato fields. Therefore, although a potential threat for these crops, MeRV infection in pepper and tomato is rare in the surveyed areas.

Multivariate models indicated that host plant density was the major predictor of MeRV infection risk, with a positive association between plant density and MeRV prevalence. This relationship has been discussed in the previous section for genus-wide potyvirus prevalence. Results for MeRV also support theory attributing a key role in infection risk to host density (Keesing et al., 2006; Ostfeld and Keesing, 2012), Again, species diversity was not a good predictor of MeRV infection risk in wild mountain rue populations of evergreen oak forests and no significant association between host density/abundance of mountain rue and species richness in evergreen oak forests was found $(r \leq 0.15$; $P \geq 0.699$ ). The poor predictive power of species richness and host relative abundance could be explained in this case to the absence of alternative host species for MeRV in evergreen oak forests and to the low levels of natural variation in plant species diversity/relative abundance across a homogeneous plant community. Finally, rainfall was negatively associated with MeRV prevalence. The rainfalls regime might have an effect on host physiology (Kennelly et al., 2012), increasing host susceptibility to virus infection and therefore virus prevalence (Seabloom et al., 2009). Alternatively, higher MeRV prevalence was generally observed in summer, when rainfall is lower and density of aphids (the main vectors of potyviruses) peaks (Nebreda et al., 2004; Mondal et al., 2016). Although MeRV transmission and the specific MeRV aphid 
vector species involved have not been characterized here, these results would be compatible with the maximum activity of the associated MeRV vectors. This highlights the relevance of considering factors associated with virus transmission to fully understand the determinants of infection risk.

For the generalist virus WHMV, the best-ranked model predicting virus prevalence contained host relative abundance as the main predictor. There was a negative association between the host relative abundance and infection risk. As discussed in the previous section, this could be due to: (i) virus infection increases host mortality, (ii) the best hosts are less frequent plants, and (iii) plant isolation favours increased prevalence through attracting virus vectors. (A'Brook 1964; Way and Heathcote, 1966; Malmstrom et al., 2005a; Power et al., 2011; Prendeville et al., 2014; McLeish et al., 2017). At odds with MeRV, WHMV infection-induce mortality was not analysed, and this thesis has no data to determine whether mortality effects has a role in the negative association between host relative abundance and WHMV prevalence. On the other hand, the best hosts of WHMV in terms of prevalence level (H. lupulus, E. elaterium) are rare species in riparian forests, which could explain why WHMV prevalence is higher in host with low relative abundance. Host species that are rare at larger spatial scales (e.g., the ecosystem scale) might act as hosts when generalist viruses can infect them in the absence of other hosts within their range, or because of heterogeneity in transmission among host species (Streicker et al., 2013) and fine-scale variation in vector ecology (Hamer et al., 2011). Also, rare host species have been acknowledged as important hosts of spillover dynamics when the ecological structure of the surrounding flora and close phylogenetic relatedness are strong predictors of prevalence (Parker et al., 2015). The observation that rare hosts determine the prevalence of WHMV also agrees with other experimental analyses indicating that hosts with low abundance determine the prevalence of other generalist viruses such as CMV in other habitats of the Iberian Peninsula (McLeish et al., 2017). Interestingly, the importance of low abundant hosts for WHMV prevalence may also explain why species richness and plant biomass are secondary predictors of WHMV prevalence: higher species richness would increase the probability of rare host to appear (and thus the trend towards a positive association between virus prevalence and species richness shown in Figure 4.22). Also, the appearance 
of this host species, whose size is generally above the average of the plants in the ecosystem, would result in larger plant biomass.

Thus, the analyses of the factors driving potyvirus species-specific prevalence in evergreen oak and riparian forests highlight the importance of considering the interplay between virus host range and host ecology in order to fully understand virus epidemiology, and provide insights on the most relevant ecological determinants of virus infection dynamics.

\subsection{Potyvirus genus-wide population genetic diversity in wild ecosystems}

Potyvirus populations in riparian forests showed considerable haplotype and genetic diversity in the NIb gene, whereas the CP gene tended to be more conserved. According to the results of this thesis, recombination played a minor role in generating such genetic diversity. This would be at odds with previous analyses in several potyvirus species, which indicated that recombination was involved in the evolution of these species (e.g., Revers et al., 1996; Wylie and Jones, 2009; Kehoe et al., 2014; Yasaka et al., 2017). Notably, the selected genomic fragments have been shown to undergo very little intra- and interspecific recombination processes (Kehoe et al., 2014; Yasaka et al., 2017), which may explain the results of this thesis. Alternatively, it has been shown that in certain potyvirus species, and in other plant viruses, recombination hotspots tend to be distributed such that complete genes are exchanged (Pagán and Holmes, 2010; Yasaka et al., 2017), in what has been termed "modular evolution" (Botstein, 1980). Given that the NIb and the CP gene sequence data sets were analysed separately, recombination breakpoints inbetween genes could not be detected. Regardless of the potential causes of the lack of recombination events in the analysed potyvirus populations, it seems clear that, in the sequenced genomic fragments, the main mechanism for generating genetic diversity is mutation. Estimates of short-term nucleotide substitution rates of potyviruses were obtained in this thesis. The substitution rates reported in this work may represent over-estimates as, due to the short sampling time span considered, transitory mutations that will not be fixed in the 
virus population were considered. However, certain temporal structure was detected (Figure 4.23), and substitution rates were in the same range than those estimated for other plant viruses, and for RNA viruses (Pagán and García-Arenal, 2018) in general (Duffy et al., 2008).

The ecological factors affecting the evolution of potyviruses in riparian and evergreen oak forests were also analysed. The most important factors predicting virus evolutionary parameters were host plant relative abundance and rainfalls and, at a lesser extent, plant biomass. Host relative abundance, rainfalls and plant biomass were positively associated with potyvirus evolutionary parameters. Two virus species, CIYVV and WHMV accounted for most infections of riparian potyviruses. These two viruses have overlapping host ranges, and common hosts (i.e., Trifolium species) are abundant in riparian forests. Larger host relative abundance may reflect larger host populations, which is often associated with an increase in the plant population genetic diversity (Frankham, 1996; Leimu et al., 2006). Higher host genetic diversity has been reported to positively correlate with pathogen population genetic diversity in wild ecosystems (Rodríguez-Nevado et al., 2018), which would explain the role of host relative abundance in the potyvirus genetic diversity. In addition, a possible explanation for the positive association between rainfalls and biomass with potyvirus population genetic diversity might be that these ecological and climatic variables indirectly affect potyvirus genetic diversity, perhaps by influencing the viral vector population. For instance, larger plant biomass, which in riparian forests generally coincides with the season of higher rainfalls, has been associated with larger aphid population sizes (Mahdavi-Arab et al., 2014; Turley and Johnson, 2015). Larger population sizes may result in multiple viruliferous aphids landing on the same plant, such that plant-to-plant transmission bottlenecks, which are generally strong for plant viruses (Moury et al., 2007; Betancourt et al., 2008), would be weaker increasing virus population genetic diversity.

Finally, this thesis has, for the first time, shed light on the patterns and processes of speciation within the genus Potyvirus in wild ecosystems. In particular, our observation of a statistically significant association between phylogeny and both host specificity and geographical origin suggests that the potyvirus species present in evergreen oak and riparian forests may have 
arisen within the same host species and in a restricted geographic area, as is expected under a process of sympatric speciation. Such a predominance of sympatric speciation may be expected, given that both plants and the aphid vectors that transmit plant viruses have a limited ability to move large geographic distances and that the anthropogenic factors that would assist allopatric speciation (such as increased transportation of plants) likely have little effects on the ecosystems analysed that have not undergone human management. However, it is important to note that the lack of information on the host range of the potyviruses present in the studied ecosystems in other geographical regions/habitats, particularly for the newly identified virus species, may be biasing this analysis against the detection of allopatric processes. Equivalent phylogenetic studies of speciation processes in other families of RNA viruses have proven to be informative, and mostly indicated speciation through sympatric processes both for plant (Pagán and Holmes, 2010) and animal (Kitchen et al., 2011) hosts.

\subsection{Potyvirus species-specific population genetic diversity in wild ecosystems}

Changes in MeRV prevalence were accompanied by variations in the virus population genetic and haplotype diversities. Viral populations may modify their genetic diversity by changing fixation rates, population sizes, and/or selection pressures (Moya et al., 2000). In this thesis, MeRV fixation rates were not estimated. However, given that the observed fluctuations in MeRV population genetic diversity occurred in short periods of time, and that genus-wide potyvirus fixation rates did not depart from values generally reported for RNA viruses, it is unlikely that these fluctuations are associated with changes in fixation rates. In turn, multiple regression model analyses indicated that MeRV prevalence was the best predictor of population genetic/haplotype diversity (higher virus genetic/haplotype diversities were observed at increasing prevalence). Higher virus prevalence results in increasing pathogen population sizes (Burdon and Thrall, 2008). Therefore, our results suggest that MeRV genetic diversity is likely modulated by fluctuations in virus population size. This 
would be in agreement with theoretical elaborations predicting that higher population sizes may lead to higher genetic diversity (Scholle et al., 2013; Lanfear et al., 2014). Interestingly, MeRV prevalence was the best predictor of $d_{S}$, both variables showing a positive association, but not of $d_{N}$. Accordingly, selection pressures, measured as $d_{N} / d_{S}$, were negatively associated with virus prevalence. This indicates that neutral evolution, rather than adaptive selection, might be responsible for the changes in MeRV population genetic diversity associated with fluctuations in virus prevalence. Similar results have been obtained in other plant virus populations infecting wild hosts (Lima et al., 2013; Rodelo-Urrego et al., 2015).

The results of the model selection analyses for WHMV population genetic diversity are more difficult to interpret. The most important factors predicting virus population haplotype and genetic diversity were host plant relative abundance and rainfalls. In addition, the best predictor of $d_{N}, d_{S}$ and $d_{N} / d_{S}$ was plant biomass (Table 4.37). At odds with the associations at the genus level found for riparian potyviruses, host relative abundance was negatively associated with WHMV genetic diversity parameters. On the other hand, rainfalls and plant biomass where positively associated with this virus population trait. As stated in the previous section, a possible explanation for these results is that the ecological and climatic variables identified in model selection analyses do not directly affect virus genetic diversity. Rather, host relative abundance, rainfalls and biomass may indirectly affect WHMV genetic diversity through influencing the viral vector population. Larger plant biomass and higher rainfalls would promote larger aphid population sizes (Mahdavi-Arab et al., 2014; Turley and Johnson, 2015), which may result in multiple viruliferous aphids landing on the same plant, such that plant-to-plant transmission bottlenecks would be weaker, increasing virus population genetic diversity without necessarily affecting virus prevalence (Bergstrom et al., 1999). This would also explain the general low predictive power of virus prevalence on population genetic diversity parameters. Note that, as stated above, the most relevant WHMV hosts are rare species in riparian forests, which, for a given number of viruliferous aphids and all else being equal, would increase the chances of multiple landings in the same host plant. This would be also compatible with the higher virus genetic diversity observed in the less abundant 
hosts (Figure 4.31). However, it must be acknowledged that all these hypotheses are highly speculative, as it is out of the scope of this thesis to analyse the relationship between vector population traits and potyvirus population genetic diversity. In all these models, WHMV prevalence was a secondary predictor, suggesting that virus population size is not a primary determinant of WHMV evolution. Moreover, when WHMV prevalence affected virus population genetic diversity (specifically $d_{N}$ ) or selection pressures $\left(d_{N} / d_{s}\right)$, the association between these traits was negative, suggesting that WHMV hosts exert a strong purifying selection on the virus population.

Finally, and despite differences in the ecology and epidemiology of MeRV and WHMV, the analysis of their speciation modes also indicated that these virus species diverged through processes of sympatric speciation, suggesting again that this might be a general trend among potyviruses. Further analyses in other potyvirus species, and including both wild and cultivated hosts, would help to test the generality of this conclusion.

\subsection{Limitations of the study}

Some cautionary comments, however, are called upon the results of this thesis. First, ML models were constructed with data from a single sampling cycle and validated with data from a second one. This is a short life span and might be well possible that yearly variation in ecological and climatic conditions not reflected in this study could reduce the accuracy of the ML models developed here. However, the models built with data of the first sampling cycle performed well in the data from the second sampling cycle, which showed seasonal variation in ecological parameters as compared with the first sampling cycle. Moreover, preliminary analyses with data of a third sampling cycle also indicated good performance of the ML models. Thus, although the predictive models constructed during this thesis can be clearly improved, they appear to be robust against yearly variation in ecological and climatic conditions. Second, the analyses of the association between potyvirus prevalence and ecological factors are based on the data from ten locations, and the analyses of the association between potyvirus evolutionary parameters and 
ecological/epidemiological factors are based on data from a subset of these locations. We are aware that this might represent a small sample size. However, it was enough to detect significant, and in many cases strong, correlations between the studied parameters. Third, although we conclude that MeRV is a specialist virus and WHMV is a generalist virus, we cannot discard that these viruses are able to infect host species that, due to their low frequency, have been insufficiently sampled. We believe, however, that this is unlikely given the sampling effort done in this work (over 10,000 samples). Fourth, although the best-ranked models explained a large proportion of the variation in potyvirus infection risk and population genetic diversity, other factors not considered here could also play a role in MeRV evolution and epidemiology. For instance, host genetic diversity or vector population dynamics has been also reported as important determinants of virus disease risk and population genetic diversity in wild ecosystems (Pagán et al., 2012; Rodelo-Urrego et al., 2015; Rodríguez-Nevado et al., 2018). Unfortunately, the lack of information on the genomic sequence of most potyvirus hosts identified in this thesis prevented including this variable in our analyses. Analyses in other host-pathogen system would help to tests the generality of our observations. Fifth, the analysis of the effect of MeRV infection on host mortality is based on the differential virus prevalence between adult and young plants. It should be noted that these results could be also explained if virus titter in adult plants would be much lower than in younger ones, such that it could lead to an underestimation of virus prevalence in adults. However, no significant differences in MeRV accumulation were observed between young and adult plants $\left(F_{1,8}=0.63 ; P=0.450\right)$.

\subsection{Concluding remarks}

In summary, this thesis analyses the ecological and climatic factors that determine the prevalence and genetic diversity of potyviruses in two wild ecosystems of the Iberian Peninsula. These analyses are based on a detailed characterization of the ecosystems and virus populations under study. The results indicate that the floristic composition of the ecosystem, and the relative abundance and the density of hosts play a fundamental role in the prevalence 
of potyviruses. The former factor determines the entry of potyviruses in the ecosystem, whereas the later two factors play a role in the extent of the infection (i.e., in the level of prevalence). Thus, these ecological factors play a role in potyvirus emergence at different stages of this process. The host range of each potyvirus species determines the relative importance of each of these ecological factors. Host relative abundance and density are also important determinants of the level of genetic diversification in potyvirus populations, which suggest a link between the epidemiological changes that lead to virus emergence and genetic changes in the virus populations. The results of this thesis also indicate that other ecological and climatic factors, perhaps linked to the dynamics of the virus vector populations, play an important role in the evolution of potyvirus populations in wild ecosystems. Finally, in the studied ecosystems the process of genetic diversification of potyviruses is compatible with sympatric speciation events. Altogether, the results of this thesis provide noble information on the determinants of plant virus emergence in wild ecosystems, and highlight the importance of considering the interplay between the ecology, epidemiology and genetic diversity of plant host and virus populations in order to fully understand plant virus emergence. 
6. CONCLUSIONS 
This thesis analysed the ecological determinants of the infection risk of species of the genus Potyvirus present in riparian and evergreen oak forests of the Iberian Peninsula. These analyses aimed at understanding the ecological determinants of plant virus emergence in wild ecosystems, which are central to fully understand plant-virus interactions but are still poorly characterized.

1. The locations monitored in this thesis conform to the ecological characteristics of typical riparian and evergreen oak forests of the lberian Peninsula and are therefore, representative of these wild ecosystems. A sampling effort of 200 plants/location is in general sufficient to fully capture the floristic composition of each location.

2. Virus prevalence was generally higher in riparian than in evergreen oak forests, which was associated with higher plant species richness and abundance of host plants, most of which were perennial species. This result supports the "Amplification Effect" hypothesis and highlights the importance of considering the identity of the species present in an ecosystem, and not only the number of plant species, in order to characterize diversity-prevalence relationships.

3. The plant community composition determines the risk of potyvirus jump into the studied wild ecosystems. Once introduced in the ecosystem, host relative abundance/density chiefly predict the extent of potyvirus invasion (prevalence), likely affecting plant-to-plant transmission. As these two processes are linked to different stages of pathogen emergence, different components of plant diversity (identity/relative abundance) determine different stages of emergence.

4. The main ecological factors that determine potyvirus prevalence in the studied wild ecosystems depend on the virus host range. The prevalence of a strict specialist is mostly determined by the density of host plants. On the other hand, the prevalence of a generalist is determined by the relative abundance of hosts. Thus, the epidemiology of potyviruses in their native wild ecosystems depends on virus life history traits. 
5. The ecological factors that determine the population genetic diversity of the potyviruses detected in riparian and evergreen oak forests at the genus-wide level are the same than those determining virus epidemiology. Thus, the ecological factors that affect potyvirus epidemiology promote genetic modifications in the virus population.

6. Higher population genetic diversity of a specialist potyvirus native of wild ecosystems is associated with higher virus prevalence. This larger genetic diversity appears to be due to larger accumulation of synonymous mutations. This suggests that neutral evolution, perhaps associated with virus population size, rather than host adaptation likely determines the genetic diversity of a specialist potyvirus.

7. The population genetic diversity of a generalist potyvirus increases with lower host relative abundance, and higher rainfalls and biomass. There is no obvious mechanism by which these factors may directly affect virus genetic diversity. However, it can be hypothesized that host relative abundance, rainfalls and biomass may indirectly affect virus genetic diversity through influencing the viral vector population.

8. The patterns of genus-wide and species-specific genetic diversification of potyviruses in wild ecosystems are compatible with sympatric speciation. 
7. BIBLIOGRAPHY 
A'Brook, J. (1964). The effect of planting date and spacing on the incidence of groundnut rosette disease and of the vector, Aphis craccivora Koch, at Mokwa, Northern Nigeria. Annals of Applied Biology, 54, 199-208.

Acosta-Leal, R., Duffy, S., Xiong, Z., Hammond, R.W., and Elena, S.F. (2011). Advances in plant virus evolution: translating evolutionary insights into better disease management. Phytopathology 101, 1136-1148.

Adams, M.J., Antoniw, J.F., and Fauquet, C.M. (2005). Molecular criteria for genus and species discrimination within the family Potyviridae. Archives of Virology 150, 459-479.

Alexander, H.M. (1984). Spatial patterns of disease induced by Fusarium moniliforme var. subglutinans in a population of Plantago lanceolata. Oecologia 62, 141143.

Alexander, H.M., Bruns, E., Schebor, H., and Malmstrom, C.M. (2017). Cropassociated virus infection in a native perennial grass: reduction in plant fitness and dynamic patterns of virus detection. Journal of Ecology 105, 1021-1031.

Alexander, H.M., Mauck, K.E., Whitfield, A.E., Garrett, K.A., and Malmstrom, C.M. (2014). Plant-virus interactions and the agro-ecological interface. European Journal of Plant Pathology 138, 529-547.

Anderson, P.K., Cunningham, A.A., Patel, N.G., Morales, F.J., Epstein, P.R., and Daszak, P. (2004). Emerging infectious diseases of plants: pathogen pollution, climate change and agrotechnology drivers. Trends in Ecology \& Evolution 19, 535-544.

Anderson, R.M., and May, R.M. (1982). Coevolution of hosts and parasites. Parasitology 85, 411-426.

Andrade, M., Sato, M., and Uyeda, I. (2007). Two resistance modes to Clover yellow vein virus in pea characterized by a green fluorescent protein-tagged virus. Phytopathology 97, 544-550.

Anisimova, M., Gil, M., Dufayard, J.-F., Dessimoz, C., and Gascuel, O. (2011). Survey of branch support methods demonstrates accuracy, power, and robustness of fast likelihood-based approximation schemes. Systematic Biology 60, 685-699.

Archibold, O.W. (1995). Mediterranean ecosystems. In Ecology of World Vegetation, (Springer), pp. 131-164.

Archie, E.A., Luikart, G., and Ezenwa, V.O. (2009). Infecting epidemiology with genetics: a new frontier in disease ecology. Trends in Ecology \& Evolution 24, 21-30.

Barbour, M.G., J.H. Burke, W.D. Pitts, F.S. Gilliam, and Schwartz, M.W. (1999). Terrestrial Plant Ecology, $3^{\text {rd }}$ Edition. The Benjamin/Cummings Publishing Company,Inc.,MenloPark,CA. 
Barnett, O.W. (1992). A summary of potyvirus taxonomy and definitions. In Potyvirus Taxonomy, (Springer), pp. 435-444.

Beauchemin, C., Boutet, N., and Laliberté, J.-F. (2007). Visualization of the interaction between the precursors of VPg, the viral protein linked to the genome of Turnip mosaic virus, and the translation eukaryotic initiation factor iso $4 \mathrm{E}$ in planta. Journal of Virology $81,775-782$.

Bergstrom, C.T., McElhany, P., and Real, L.A. (1999). Transmission bottlenecks as determinants of virulence in rapidly evolving pathogens. Proceedings of the National Academy of Sciences 96, 5095-5100.

Betancourt, M., Fereres, A., Fraile, A., and García-Arenal, F. (2008). Estimation of the effective number of founders that initiate an infection after aphid transmission of a multipartite plant virus. Journal of Virology 82, 12416-12421.

Bhat, S., Folimonova, S.Y., Cole, A.B., Ballard, K.D., Lei, Z., Watson, B.S., Sumner, L.W., and Nelson, R.S. (2013). Influence of host chloroplast proteins on Tobacco mosaic virus accumulation and intercellular movement. Plant Physiology 161, 134-147.

Blanco, E., Casado, M.A., Costa, M., Escribano, R., García, M., Génova, M., Gómez, A., Gómez, F., Moreno, J.C., Morla, C., et al. (2005). Los bosques ibéricos. Una interpretación geobotánica, $4^{\text {th }}$ Edition. Editorial Planeta, Barcelona.

Boots, M., and Bowers, R.G. (2004). The evolution of resistance through costly acquired immunity. Proceedings of the Royal Society B: Biological Sciences $271,715-723$.

Borer, E.T., Mitchell, C.E., Power, A.G., and Seabloom, E.W. (2009). Consumers indirectly increase infection risk in grassland food webs. Proceedings of the National Academy of Sciences 106, 503-506.

Borer, E.T., Seabloom, E.W., Gruner, D.S., Harpole, W.S., Hillebrand, H., Lind, E.M., Adler, P.B., Alberti, J., Anderson, T.M., and Bakker, J.D. (2014a). Herbivores and nutrients control grassland plant diversity via light limitation. Nature 508, 517.

Borer, E.T., Seabloom, E.W., Mitchell, C.E., and Cronin, J.P. (2014b). Multiple nutrients and herbivores interact to govern diversity, productivity, composition, and infection in a successional grassland. Oikos 123, 214-224.

Borer, E.T., Seabloom, E.W., Mitchell, C.E., and Power, A.G. (2010). Local context drives infection of grasses by vector-borne generalist viruses. Ecology Letters 13, 810-818.

Botstein, D. (1980). A theory of modular evolution for bacteriophages. Annals of the New York Academy of Sciences 354, 484-491.

Bousalem, M., Dallot, S., Fuji, S., and Natsuaki, K.T. (2003). Origin, world-wide dispersion, bio-geographical diversification, radiation and recombination: an evolutionary history of Yam mild mosaic virus (YMMV). Infection, Genetics and Evolution 3, 189-206. 
Breiman, L. (2001). Random forests. Machine Learning 45, 5-32.

Bruno, D., Belmar, O., Sánchez-Fernández, D., Guareschi, S., Millán, A., and Velasco, J. (2014). Responses of Mediterranean aquatic and riparian communities to human pressures at different spatial scales. Ecological Indicators 45, 456-464.

Bruns, E., Hood, M.E., and Antonovics, J. (2015). Rate of resistance evolution and polymorphism in long-and short-lived hosts. Evolution 69, 551-560.

Buckling, A., and Rainey, P.B. (2002). The role of parasites in sympatric and allopatric host diversification. Nature 420, 496-499.

Bueso, E., Serrano, R., Pallás, V., and Sánchez-Navarro, J.A. (2017). Seed tolerance to deterioration in arabidopsis is affected by virus infection. Plant Physiology and Biochemistry 116, 1-8.

Bujarski, J.J. (2013). Genetic recombination in plant-infecting messenger-sense RNA viruses: overview and research perspectives. Frontiers in Plant Science 4, 68.

Burdon, J.J., and Chilvers, G.A. (1982). Host density as a factor in plant disease ecology. Annual Review of Phytopathology 20,143-166.

Burdon, J.J., and Thrall, P.H. (2008). Pathogen evolution across the agro-ecological interface: implications for disease management: Pathogen evolution in agroecosystems. Evolutionary Applications 1, 57-65.

Burdon, J.J., Wennström, A., Ericson, L., Müller, W.J., and Morton, R. (1992). Densitydependent mortality in Pinus sylvestris caused by the snow blight pathogen Phacidium infestans. Oecologia 90, 74-79.

Burnham, K.P., and Anderson, D.R. (2002). Multimodel inference: understanding AIC and BIC in model selection. Sociological Methods \& Research 33, 261-304.

Bwye, A.M., Jones, R.A.C., and Proudlove, W. (1994). Effects of sowing seed with different levels of infection, plant density and the growth stage at which plants first develop symptoms on cucumber mosaic-virus infection of narrow-leafed lupins (Lupinus angustifolius). Australian Journal of Agricultural Research 45, 1395-1412.

Canto, T., and Palukaitis, P. (2002). Novel N gene-associated, temperatureindependent resistance to the movement of Tobacco mosaic virus vectors neutralized by a Cucumber mosaic virus RNA1 transgene. Journal of Virology 76, 12908-12916.

Carrasco, P., Daros, J.A., Agudelo-Romero, P., and Elena, S.F. (2007). A real-time RT-PCR assay for quantifying the fitness of Tobacco etch virus in competition experiments. Journal of Virological Methods 139, 181-188.

Carrington, J.C., Cary, S.M., Parks, T.D., and Dougherty, W.G. (1989). A second proteinase encoded by a plant potyvirus genome. The EMBO Journal $8,365-$ 370.

Castro, I., Casado, M.Á., Ramírez-Sanz, L., de Miguel, J.M., Costa, M., and Pineda, F.D. (1996). Funciones de estimación de la biomasa aérea de varias especies 
del matorral mediterráneo del centro de la península lbérica. Orsis: Organismes i Sistemes 11, 107-116.

Chang, S., Puryear, J., and Cairney, J. (1993). A simple and efficient method for isolating RNA from pine trees. Plant Molecular Biology Reporter 11, 113-116.

Chao, L. (1990). Fitness of RNA virus decreased by Muller's ratchet. Nature 348, 454.

Chappell, T.M., Beaudoin, A.L., and Kennedy, G.G. (2013). Interacting virus abundance and transmission intensity underlie tomato spotted wilt virus incidence: an example weather-based model for cultivated tobacco. PloS One 8, e73321.

Chare, E.R., and Holmes, E.C. (2006). A phylogenetic survey of recombination frequency in plant RNA viruses. Archives of Virology 151, 933-946.

Chatzivassiliou, E.K., Papapanagiotou, A.P., Mpenardis, P.D., Perdikis, D.C., and Menexes, G. (2016). Transmission of Moroccan watermelon mosaic virus (MWMV) by Aphids in Greece. Plant Disease 100, 601-606.

Chawla, N.V., Bowyer, K.W., Hall, L.O., and Kegelmeyer, W.P. (2002). SMOTE: synthetic minority over-sampling technique. Journal of Artificial Intelligence Research 16, 321-357.

Chung, B.Y.-W., Miller, W.A., Atkins, J.F., and Firth, A.E. (2008). An overlapping essential gene in the Potyviridae. Proceedings of the National Academy of Sciences 105, 5897-5902.

Civitello, D.J., Cohen, J., Fatima, H., Halstead, N.T., Liriano, J., McMahon, T.A., Ortega, C.N., Sauer, E.L., Sehgal, T., and Young, S. (2015). Biodiversity inhibits parasites: broad evidence for the dilution effect. Proceedings of the National Academy of Sciences 112, 8667-8671.

Clark, C.A., Davis, J.A., Abad, J.A., Cuellar, W.J., Fuentes, S., Kreuze, J.F., Gibson, R.W., Mukasa, S.B., Tugume, A.K., and Tairo, F.D. (2012). Sweetpotato viruses: 15 years of progress on understanding and managing complex diseases. Plant Disease 96, 168-185.

Cobb, R.C., Meentemeyer, R.K., and Rizzo, D.M. (2010). Apparent competition in canopy trees determined by pathogen transmission rather than susceptibility. Ecology 91, 327-333.

Colwell, R.K. (2006). EstimateS: statistical estimation of species richness and shared species from simples, version 8.0. Http://Purl. Oclc. Org/Estimates.

Cooper, I., and Jones, R.A. (2006). Wild plants and viruses: under-investigated ecosystems. Advances in Virus Research 67, 1-47.

Costa, A., Madeira, M., Lima Santos, J., and Oliveira, Â. (2011). Change and dynamics in Mediterranean evergreen oak woodlands landscapes of Southwestern Iberian Peninsula. Landscape and Urban Planning 102, 164176. 
Cotton, S., Grangeon, R., Thivierge, K., Mathieu, I., Ide, C., Wei, T., Wang, A., and Laliberté, J.-F. (2009). Turnip mosaic virus RNA replication complex vesicles are mobile, align with microfilaments, and are each derived from a single viral genome. Journal of Virology 83, 10460-10471.

Cui, X., Wei, T., Chowda-Reddy, R.V., Sun, G., and Wang, A. (2010). The Tobacco etch virus $\mathrm{P} 3$ protein forms mobile inclusions via the early secretory pathway and traffics along actin microfilaments. Virology 397, 56-63.

Culbreath, A.K., and Srinivasan, R. (2011). Epidemiology of spotted wilt disease of peanut caused by Tomato spotted wilt virus in the southeastern US. Virus Research 159, 101-109.

da Silva, S.J., Castillo-Urquiza, G.P., Júnior, B.T.H., Assunção, I.P., Lima, G.S., PioRibeiro, G., Mizubuti, E.S., and Zerbini, F.M. (2011). High genetic variability and recombination in a begomovirus population infecting the ubiquitous weed Cleome affinis in northeastern Brazil. Archives of Virology 156, 2205-2213.

Darriba, D., Taboada, G.L., Doallo, R., and Posada, D. (2012). jModelTest 2: more models, new heuristics and parallel computing. Nature Methods 9, 772-772.

Dedryver, C.-A., Le Ralec, A., and Fabre, F. (2010). The conflicting relationships between aphids and men: a review of aphid damage and control strategies. Comptes Rendus Biologies 333, 539-553.

Delwart, E.L. (2007). Viral metagenomics. Reviews in Medical Virology 17, 115-131.

Desbiez, C., and Lecoq, H. (2004). The nucleotide sequence of Watermelon mosaic virus (WMV, Potyvirus) reveals interspecific recombination between two related potyviruses in the $5^{\prime}$ part of the genome. Archives of Virology 149, 1619-1632.

Desbiez, C., Joannon, B., Wipf-Scheibel, C., Chandeysson, C., and Lecoq, H. (2009). Emergence of new strains of Watermelon mosaic virus in South-eastern France: Evidence for limited spread but rapid local population shift. Virus Research 141, 201-208.

Desbiez, C., Schoeny, A., Maisonneuve, B., Berthier, K., Bornard, I., Chandeysson, C., Fabre, F., Girardot, G., Gognalons, P., and Lecoq, H. (2017). Molecular and biological characterization of two potyviruses infecting lettuce in southeastern France. Plant Pathology 66, 970-979.

Desbiez, C., Wipf-Scheibel, C., Millot, P., Verdin, E., Dafalla, G., and Lecoq, H. (2017). New species in the papaya ringspot virus cluster: Insights into the evolution of the PRSV lineage. Virus Research 241, 88-94.

Diaz, B.M., and Fereres, A. (2005). Life table and population parameters of Nasonovia ribisnigri (Homoptera: Aphididae) at different constant temperatures. Environmental Entomology 34, 527-534.

Díaz-Pendón, J.A., Cañizares, M.C., Moriones, E., Bejarano, E.R., Czosnek, H., and Navas-Castillo, J. (2010). Tomato yellow leaf curl viruses: ménage à trois between the virus complex, the plant and the whitefly vector. Molecular Plant Pathology 11, 441-450. 
Dietterich, T.G. (1998). Approximate statistical tests for comparing supervised classification learning algorithms. Neural Computation 10, 1895-1923.

Dogimont, C., Bendahmane, A., Chovelon, V., and Boissot, N. (2010). Host plant resistance to aphids in cultivated crops: genetic and molecular bases, and interactions with aphid populations. Comptes Rendus Biologies 333, 566-573.

Dolja, V.V., Haldeman-Cahill, R., Montgomery, A.E., Vandenbosch, K.A., and Carrington, J.C. (1995). Capsid protein determinants involved in cell-to-cell and long distance movement of tobacco etch potyvirus. Virology 206, 1007-1016.

Dolja, V.V., Haldeman, R., Robertson, N.L., Dougherty, W.G., and Carrington, J.C. (1994). Distinct functions of capsid protein in assembly and movement of tobacco etch potyvirus in plants. The EMBO Journal 13, 1482.

dos Santos Martins, D., Ventura, J.A., Rita de Cássia, A.L., Fornazier, M.J., Rezende, J.A., Culik, M.P., Ferreira, P.S.F., Peronti, A.L.B., de Carvalho, R.C.Z., and Sousa-Silva, C.R. (2016). Aphid vectors of Papaya ringspot virus and their weed hosts in orchards in the major papaya producing and exporting region of Brazil. Crop Protection 90, 191-196.

Dougherty, W.G., and Parks, T.D. (1991). Post-translational processing of the Tobacco etch virus 49-kDa small nuclear inclusion polyprotein: identification of an internal cleavage site and delimitation of VPg and proteinase domains. Virology 183, 449-456.

Drake, J.W., and Holland, J.J. (1999). Mutation rates among RNA viruses. Proceedings of the National Academy of Sciences 96, 13910-13913.

Drummond, A.J., and Rambaut, A. (2007). BEAST: Bayesian evolutionary analysis by sampling trees. BMC Evolutionary Biology 7, 214.

Drummond, A.J., Ho, S.Y., Phillips, M.J., and Rambaut, A. (2006). Relaxed phylogenetics and dating with confidence. PLoS Biology 4, e88.

Duffy, S., Shackelton, L.A., and Holmes, E.C. (2008). Rates of evolutionary change in viruses: patterns and determinants. Nature Reviews Genetics 9, 267.

Edgar, R.C. (2004). MUSCLE: multiple sequence alignment with high accuracy and high throughput. Nucleic Acids Research 32, 1792-1797.

Elena, S.F., and Sanjuán, R. (2005). Adaptive value of high mutation rates of RNA viruses: separating causes from consequences. Journal of Virology 79, 1155511558.

Elena, S.F., Bedhomme, S., Carrasco, P., Cuevas, J.M., de la Iglesia, F., Lafforgue, G., Lalić, J., Pròsper, À., Tromas, N., and Zwart, M.P. (2011). The evolutionary genetics of emerging plant RNA viruses. Molecular Plant-Microbe Interactions 24, 287-293.

Elena, S.F., Fraile, A., and García-Arenal, F. (2014). Evolution and emergence of plant viruses. Advances in Virus Research 88, 161-191. 
Fabre, F., Moury, B., Johansen, E.I., Simon, V., Jacquemond, M., and Senoussi, R. (2014). Narrow bottlenecks affect Pea seedborne mosaic virus populations during vertical seed transmission but not during leaf colonization. PLoS Pathogens 10, e1003833.

Fajinmi, A.A., Odebode, C.A., and Fajinmi, O.B. (2011). The effect of agro-ecological zones on the incidence and distribution of aphid vectors of Pepper veinal mottle virus, on cultivated pepper (Capsicum annuum L.) in Nigeria. Journal of Central European Agriculture 12.

Fargette, D., Konate, G., Fauquet, C., Muller, E., Peterschmitt, M., and Thresh, J.M. (2006). Molecular ecology and emergence of tropical plant viruses. Annual Review of Phytopathology 44, 235-260.

Fargette, D., Pinel, A., Rakotomalala, M., Sangu, E., Traoré, O., Sérémé, D., Sorho, F., Issaka, S., Hébrard, E., and Séré, Y. (2008). Rice yellow mottle virus, an RNA plant virus, evolves as rapidly as most RNA animal viruses. Journal of Virology 82, 3584-3589.

Fawcett, T. (2004). ROC graphs: Notes and practical considerations for researchers. Machine Learning 31, 1-38.

Fereres, A., Perez, P., Gemeno, C., and Ponz, F. (1993). Transmission of Spanish pepper-and potato-PVY isolates by aphid (Homoptera: Aphididae) vectors: epidemiological implications. Environmental Entomology 22, 1260-1265.

Fernández, A., Guo, H.S., Sáenz, P., Simón-Buela, L., de Cedrón, M.G., and García, J.A. (1997). The motif $V$ of plum pox potyvirus $C I$ RNA helicase is involved in NTP hydrolysis and is essential for virus RNA replication. Nucleic Acids Research 25, 4474-4480.

Fraile, A., and García-Arenal, F. (2016). Environment and evolution modulate plant virus pathogenesis. Current Opinion in Virology 17, 50-56.

Fraile, A., Hily, J.-M., Pagán, I., Pacios, L.F., and García-Arenal, F. (2014). Host resistance selects for traits unrelated to resistance-breaking that affect fitness in a plant virus. Molecular Biology and Evolution 31, 928-939.

Fraile, A., McLeish, M.J., Pagán, I., González-Jara, P., Piñero, D., and García-Arenal, F. (2017). Environmental heterogeneity and the evolution of plant-virus interactions: Viruses in wild pepper populations. Virus Research 241, 68-76.

Frankham, R. (1996). Relationship of genetic variation to population size in wildlife. Conservation Biology 10, 1500-1508.

French, R., and Stenger, D.C. (2003). Evolution of Wheat streak mosaic virus: dynamics of population growth within plants may explain limited variation. Annual Review of Phytopathology 41, 199-214.

Froissart, R., Roze, D., Uzest, M., Galibert, L., Blanc, S., and Michalakis, Y. (2005). Recombination every day: abundant recombination in a virus during a single multi-cellular host infection. PLoS Biology 3, e89. 
Funayama, S., Terashima, I., and Yahara, T. (2001). Effects of virus infection and light environment on population dynamics of Eupatorium makinoi (Asteraceae). American Journal of Botany 88, 616-622.

Funayama-Noguchi, S. (2001). Ecophysiology of virus-infected plants: a case study of Eupatorium makinoi infected by geminivirus. Plant Biology 3, 251-262.

Futuyma, D.J., and Moreno, G. (1988). The evolution of ecological specialization. Annual Review of Ecology and Systematics 19, 207-233.

García, J.A., Glasa, M., Cambra, M., and Candresse, T. (2014). Plum pox virus and sharka: a model potyvirus and a major disease. Molecular Plant Pathology 15, 226-241.

García-Arenal, F., and Fraile, A. (2013). Trade-offs in host range evolution of plant viruses. Plant Pathology 62, 2-9.

García-Arenal, F., Fraile, A., and Malpica, J.M. (2001). Variability and genetic structure of plant virus populations. Annual Review of Phytopathology 39, 157-186.

Garrett, K.A., Dendy, S.P., Frank, E.E., Rouse, M.N., and Travers, S.E. (2006). Climate change effects on plant disease: genomes to ecosystems. Annual Review of Phytopathology 44, 489-509.

Gavilán, R.G. (2005). The use of climatic parameters and indices in vegetation distribution. A case study in the Spanish Sistema Central. International Journal of Biometeorology 50, 111-120.

Gavilán, R., and Fernández-González, F. (1997). Climatic discrimination of Mediterranean broad-leaved sclerophyllous and deciduous forests in central Spain. Journal of Vegetation Science 8, 377-386.

Gavilán, R.G., Fernández-González, F., and Blasi, C. (1998). Climatic classification and ordination of the Spanish Sistema Central: relationships with potential vegetation. Plant Ecology 139, 1-11.

Gell, G., Sebestyén, E., and Balázs, E. (2015). Recombination analysis of Maize dwarf mosaic virus (MDMV) in the Sugarcane mosaic virus (SCMV) subgroup of potyviruses. Virus Genes 50, 79-86.

Gibbs, A.J., Ohshima, K., Phillips, M.J., and Gibbs, M.J. (2008). The prehistory of potyviruses: their initial radiation was during the dawn of agriculture. PLoS One 3, e2523.

Gilbert, G.S. (2002). Evolutionary ecology of plant diseases in natural ecosystems. Annual Review of Phytopathology 40, 13-43.

Giraud, T., Gladieux, P., and Gavrilets, S. (2010). Linking the emergence of fungal plant diseases with ecological speciation. Trends in Ecology \& Evolution 25, 387-395.

Gómez, P., Rodríguez-Hernández, A.M., Moury, B., and Aranda, M.A. (2009). Genetic resistance for the sustainable control of plant virus diseases: breeding, mechanisms and durability. European Journal of Plant Pathology 125, 1-22. 
Gómez, P., Sempere, R., and Aranda, M.A. (2012). Pepino mosaic virus and Tomato torrado virus: Two emerging viruses affecting tomato crops in the Mediterranean basin. In Advances in Virus Research, (Elsevier), pp. 505-532.

Gotelli, N.J., and Chao, A. (2013). Measuring and estimating species richness, species diversity, and biotic similarity from sampling data. In Encyclopedia of Biodiversity, (Academic Press), pp. 195-211

Gotelli, N.J., and R.K. Colwell. (2010). Biological Diversity: Frontiers In Measurement And Assessment. Oxford University Press, Oxford.

Grenfell, B.T., Pybus, O.G., Gog, J.R., Wood, J.L., Daly, J.M., Mumford, J.A., and Holmes, E.C. (2004). Unifying the epidemiological and evolutionary dynamics of pathogens. Science 303, 327-332.

Guindon, S., Dufayard, J.-F., Lefort, V., Anisimova, M., Hordijk, W., and Gascuel, O. (2010). New algorithms and methods to estimate maximum-likelihood phylogenies: assessing the performance of PhyML 3.0. Systematic Biology 59, 307-321.

Gutiérrez, S., Yvon, M., Pirolles, E., Garzo, E., Fereres, A., Michalakis, Y., and Blanc, S. (2012). Circulating virus load determines the size of bottlenecks in viral populations progressing within a host. PLoS Pathogens 8, e1003009.

Ha, C., Coombs, S., Revill, P.A., Harding, R.M., Vu, M., and Dale, J.L. (2008). Design and application of two novel degenerate primer pairs for the detection and complete genomic characterization of potyviruses. Archives of Virology 153, 25-36.

Haas, S.E., Hooten, M.B., Rizzo, D.M., and Meentemeyer, R.K. (2011). Forest species diversity reduces disease risk in a generalist plant pathogen invasion. Ecology Letters 14, 1108-1116.

Hagiwara-Komoda, Y., Choi, S.H., Sato, M., Atsumi, G., Abe, J., Fukuda, J., Honjo, M.N., Nagano, A.J., Komoda, K., and Nakahara, K.S. (2016). Truncated yet functional viral protein produced via RNA polymerase slippage implies underestimated coding capacity of RNA viruses. Scientific Reports 6, 21411.

Hajimorad, M.R., Wen, R.-H., Eggenberger, A.L., Hill, J.H., and Maroof, M.S. (2011). Experimental adaptation of an RNA virus mimics natural evolution. Journal of Virology 85, 2557-2564.

Hamer, G.L., Chaves, L.F., Anderson, T.K., Kitron, U.D., Brawn, J.D., Ruiz, M.O., Loss, S.R., Walker, E.D., and Goldberg, T.L. (2011). Fine-scale variation in vector host use and force of infection drive localized patterns of West Nile virus transmission. PloS One 6, e23767.

Hanssen, I.M., and Thomma, B.P. (2010). Pepino mosaic virus: a successful pathogen that rapidly evolved from emerging to endemic in tomato crops. Molecular Plant Pathology 11, 179-189.

Hanssen, I.M., Lapidot, M., and Thomma, B.P. (2010). Emerging viral diseases of tomato crops. Molecular Plant-Microbe Interactions 23, 539-548. 
Harrison, B.D. (1956). The infectivity of extracts made from leaves at intervals after inoculation with viruses. Microbiology 15, 210-220.

Hartl, D.L., and Clark, A.G. (2007). Principles of Population Genetics. $3^{\text {rd }}$ Edition. Sinauer Associates, Inc, Sunderland, MA.

Harvell, C.D., Mitchell, C.E., Ward, J.R., Altizer, S., Dobson, A.P., Ostfeld, R.S., and Samuel, M.D. (2002). Climate warming and disease risks for terrestrial and marine biota. Science 296, 2158-2162.

Herms, D.A., and Mattson, W.J. (1992). The dilemma of plants: to grow or defend. The Quarterly Review of Biology 67, 283-335.

Hillung, J., Cuevas, J.M., and Elena, S.F. (2015). Evaluating the within-host fitness effects of mutations fixed during virus adaptation to different ecotypes of a new host. Phil. Trans. R. Soc. B 370, 20140292.

Hily, J.M., García, A., Moreno, A., Plaza, M., Wilkinson, M.D., Fereres, A., Fraile, A., and García-Arenal, F. (2014). The relationship between host lifespan and pathogen reservoir potential: an analysis in the system Arabidopsis thalianaCucumber mosaic virus. PLoS Pathogens 10, e1004492.

Hobbs, H.A., Eastburn, D.M., D’Arcy, C.J., Kindhart, J.D., Masiunas, J.B., Voegtlin, D.J., Weinzierl, R.A., and McCoppin, N.K. (2000). Solanaceous weeds as possible sources of Cucumber mosaic virus in southern Illinois for aphid transmission to pepper. Plant Disease 84, 1221-1224.

Holmes, E.C. (2009). The evolution and emergence of RNA viruses. Oxford University Press.

Hong, Y., and Hunt, A.G. (1996). RNA polymerase activity catalyzed by a potyvirusencoded RNA-dependent RNA polymerase. Virology 226, 146-151.

Hosmer Jr, D.W., Lemeshow, S., and Sturdivant, R.X. (2013). Logistic regression models for multinomial and ordinal outcomes. In Applied Logistic Regression (Wiley), pp. 269-311.

Hsu, H.T. (2002). Biological control of plant pathogens (Viruses). Encyclopedia of Pest Management (Marcel Dekker, New York, 68pp).

Hudson, P.J., Perkins, S.E., and Cattadori, I.M. (2008). The emergence of wildlife disease and the application of ecology. In Infectious Disease Ecology: The Effects of Ecosystems on Disease and of Disease on Ecosystems. 11th Cary Conference, Millbrook, New York, USA, May 2005., (Princeton University Press), pp. 347-367.

Hughes, A.L. (2009). Small effective population sizes and rare nonsynonymous variants in potyviruses. Virology 393, 127-134.

Hull, R. (2014). Plant Virology $5^{\text {th }}$ Ed. Academic Press, San Diego.

Hurwitz, B.L., and Sullivan, M.B. (2013). The Pacific Ocean Virome (POV): a marine viral metagenomic dataset and associated protein clusters for quantitative viral ecology. PloS One 8, e57355. 
Huyse, T., Poulin, R., and Theron, A. (2005). Speciation in parasites: a population genetics approach. Trends in Parasitology 21, 469-475.

Johnson, P.T., Ostfeld, R.S., and Keesing, F. (2015). Frontiers in research on biodiversity and disease. Ecology Letters 18, 1119-1133.

Johnson, P.T., Rohr, J.R., Hoverman, J.T., Kellermanns, E., Bowerman, J., and Lunde, K.B. (2012). Living fast and dying of infection: host life history drives interspecific variation in infection and disease risk. Ecology Letters 15, 235242.

Jones, R.A.C. (2009). Plant virus emergence and evolution: origins, new encounter scenarios, factors driving emergence, effects of changing world conditions, and prospects for control. Virus Research. 141, 113-130.

Kassanis, B. (1957). Effects of changing temperature on plant virus diseases. In Advances in Virus Research, (Elsevier), pp. 221-241.

Kasschau, K.D., and Carrington, J.C. (1998). A counterdefensive strategy of plant viruses: suppression of posttranscriptional gene silencing. Cell 95, 461-470.

Kasschau, K.D., Xie, Z., Allen, E., Llave, C., Chapman, E.J., Krizan, K.A., and Carrington, J.C. (2003). P1/HC-Pro, a viral suppressor of RNA silencing, interferes with Arabidopsis development and miRNA function. Developmental Cell 4, 205-217.

Katis, N.I., Tsitsipis, J.A., Lykouressis, D.P., Papapanayotou, A., Margaritopoulos, J.T., Kokinis, G.M., Perdikis, D.C., and Manoussopoulos, I.N. (2006). Transmission of Zucchini yellow mosaic virus by colonizing and non-colonizing aphids in Greece and new aphid species vectors of the virus. Journal of Phytopathology 154, 293-302.

Katoh, K., Misawa, K., Kuma, K., and Miyata, T. (2002). MAFFT: a novel method for rapid multiple sequence alignment based on fast Fourier transform. Nucleic Acids Research 30, 3059-3066.

Kay, K.M., Whittall, J.B., and Hodges, S.A. (2006). A survey of nuclear ribosomal internal transcribed spacer substitution rates across angiosperms: an approximate molecular clock with life history effects. BMC Evolutionary Biology 6,36 .

Kearse, M., Moir, R., Wilson, A., Stones-Havas, S., Cheung, M., Sturrock, S., Buxton, S., Cooper, A., Markowitz, S., and Duran, C. (2012). Geneious Basic: an integrated and extendable desktop software platform for the organization and analysis of sequence data. Bioinformatics 28, 1647-1649.

Keesing, F., Belden, L.K., Daszak, P., Dobson, A., Harvell, C.D., Holt, R.D., Hudson, P., Jolles, A., Jones, K.E., and Mitchell, C.E. (2010). Impacts of biodiversity on the emergence and transmission of infectious diseases. Nature 468, 647.

Keesing, F., Holt, R.D., and Ostfeld, R.S. (2006). Effects of species diversity on disease risk. Ecology Letters 9, 485-498. 
Kehoe, M.A., Coutts, B.A., Buirchell, B.J., and Jones, R.A. (2014). Split personality of a Potyvirus: to specialize or not to specialize? PloS One 9, e105770.

Kennelly, M., O'Mara, J., Rivard, C., Miller, G.L., and Smith, D. (2012). Introduction to abiotic disorders in plants. The Plant Health Instructor 10, 1094.

King, A.M., Lefkowitz, E., Adams, M.J., and Carstens, E.B. (2012). Virus taxonomy: ninth report of the International Committee on Taxonomy of Viruses (Elsevier).

Kitchen, A., Shackelton, L.A., and Holmes, E.C. (2011). Family level phylogenies reveal modes of macroevolution in RNA viruses. Proceedings of the National Academy of Sciences 108, 238-243.

Knops, J.M., Tilman, D., Haddad, N.M., Naeem, S., Mitchell, C.E., Haarstad, J., Ritchie, M.E., Howe, K.M., Reich, P.B., and Siemann, E. (1999). Effects of plant species richness on invasion dynamics, disease outbreaks, insect abundances and diversity. Ecology Letters 2, 286-293.

Kosakovsky Pond, S.L., and Frost, S.D. (2005). Not so different after all: a comparison of methods for detecting amino acid sites under selection. Molecular Biology and Evolution 22, 1208-1222.

Lanfear, R., Kokko, H., and Eyre-Walker, A. (2014). Population size and the rate of evolution. Trends in Ecology \& Evolution 29, 33-41.

Langmead, B., and Salzberg, S.L. (2012). Fast gapped-read alignment with Bowtie 2. Nature Methods 9, 357-359.

Larsen, R.C., Miklas, P.N., Druffel, K.L., and Wyatt, S.D. (2005). NL-3 K strain is a stable and naturally occurring interspecific recombinant derived from Bean common mosaic necrosis virus and Bean common mosaic virus. Phytopathology 95, 1037-1042.

Larsen, R.C., Miklas, P.N., Eastwell, K.C., and Grau, C.R. (2008). A strain of Clover yellow vein virus that causes severe pod necrosis disease in snap bean. Plant Disease 92, 1026-1032.

Lecoq, H., and Raccah, B. (2001). Cross-protection: interactions between strains exploited to control plant virus diseases. In Biotic Interactions in Plant Pathogen Associations, (CAB International), pp. 177-192.

Lecoq, H., Moury, B., Desbiez, C., Palloix, A., and Pitrat, M. (2004). Durable virus resistance in plants through conventional approaches: a challenge. Virus Research 100, 31-39.

Lefeuvre, P., Martin, D.P., Harkins, G., Lemey, P., Gray, A.J., Meredith, S., Lakay, F., Monjane, A., Lett, J.-M., and Varsani, A. (2010). The spread of tomato yellow leaf curl virus from the Middle East to the world. PLoS Pathogens 6, e1001164.

Leimu, R., Mutikainen, P.I.A., Koricheva, J., and Fischer, M. (2006). How general are positive relationships between plant population size, fitness and genetic variation? Journal of Ecology 94, 942-952. 
Leonard, S., Viel, C., Beauchemin, C., Daigneault, N., Fortin, M.G., and Laliberte, J.-F. (2004). Interaction of VPg-Pro of Turnip mosaic virus with the translation initiation factor $4 \mathrm{E}$ and the poly (A)-binding protein in planta. Journal of General Virology 85, 1055-1063.

Li, F., Xu, D., Abad, J., and Li, R. (2012). Phylogenetic relationships of closely related potyviruses infecting sweet potato determined by genomic characterization of Sweet potato virus $G$ and Sweet potato virus 2. Virus Genes 45, 118-125.

Li, H., Ruan, J., and Durbin, R. (2008). Mapping short DNA sequencing reads and calling variants using mapping quality scores. Genome Research 18, 18511858.

Librado, P., and Rozas, J. (2009). DnaSP v5: a software for comprehensive analysis of DNA polymorphism data. Bioinformatics 25, 1451-1452.

Lima, A.T., Sobrinho, R.R., Gonzalez-Aguilera, J., Rocha, C.S., Silva, S.J., Xavier, C.A., Silva, F.N., Duffy, S., and Zerbini, F.M. (2013). Synonymous site variation due to recombination explains higher genetic variability in begomovirus populations infecting non-cultivated hosts. Journal of General Virology 94, 418-431.

Lindbo, J.A., and Falk, B.W. (2017). The Impact of "Coat Protein-Mediated Virus Resistance" in Applied Plant Pathology and Basic Research. Phytopathology $107,624-634$.

LoGiudice, K., Ostfeld, R.S., Schmidt, K.A., and Keesing, F. (2003). The ecology of infectious disease: effects of host diversity and community composition on Lyme disease risk. Proceedings of the National Academy of Sciences 100, 567-571.

Loidi, J. (2017). The Vegetation of the Iberian Peninsula (Springer).

Lole, K.S., Bollinger, R.C., Paranjape, R.S., Gadkari, D., Kulkarni, S.S., Novak, N.G., Ingersoll, R., Sheppard, H.W., and Ray, S.C. (1999). Full-length human immunodeficiency virus type 1 genomes from subtype C-infected seroconverters in India, with evidence of intersubtype recombination. Journal of Virology $73,152-160$.

Louppe, G., Wehenkel, L., Sutera, A., and Geurts, P. (2013). Understanding variable importances in forests of randomized trees. In Advances in Neural Information Processing Systems, pp. 431-439.

Luis-Arteaga, M., Alvarez, J.M., Alonso-Prados, J.L., Bernal, J.J., García-Arenal, F., Laviña, A., Batlle, A., and Moriones, E. (1998). Occurrence, distribution, and relative incidence of mosaic viruses infecting field-grown melon in Spain. Plant Disease 82, 979-982.

Luis-Arteaga, M., García-Arenal, F., And Rodríguez-Cerezo, E. (1996). Characterization of a strain of clover yellow vein potyvirus infecting borage (Borago officinalis L.) in Spain. Plant Pathology 45, 38-44. 
Mahdavi-Arab, N., Meyer, S.T., Mehrparvar, M., and Weisser, W.W. (2014). Complex effects of fertilization on plant and herbivore performance in the presence of a plant competitor and activated carbon. Plos One 9, e103731.

Mäkinen, K., and Hafrén, A. (2014). Intracellular coordination of potyviral RNA functions in infection. Frontiers in Plant Science 5, 110.

Malmstrom, C.M., and Alexander, H.M. (2016). Effects of crop viruses on wild plants. Current Opinion in Virology 19, 30-36.

Malmstrom, C.M., Hughes, C.C., Newton, L.A., and Stoner, C.J. (2005a). Virus infection in remnant native bunchgrasses from invaded California grasslands. New Phytologist 168, 217-230.

Malmstrom, C.M., McCullough, A.J., Johnson, H.A., Newton, L.A., and Borer, E.T. (2005b). Invasive annual grasses indirectly increase virus incidence in California native perennial bunchgrasses. Oecologia 145, 153-164.

Malmstrom, C.M., Melcher, U., and Bosque-Pérez, N.A. (2011). The expanding field of plant virus ecology: Historical foundations, knowledge gaps, and research directions. Virus Research 159, 84-94.

Malpica, J.M., Fraile, A., Moreno, I., Obies, C.I., Drake, J.W., and García-Arenal, F. (2002a). The rate and character of spontaneous mutation in an RNA virus. Genetics 162, 1505-1511.

Malpica, J.M., Fraile, A., Moreno, I., Obies, C.I., Drake, J.W., and García-Arenal, F. (2002b). The rate and character of spontaneous mutation in an RNA virus. Genetics 162, 1505-1511.

Malpica, J.M., Sacristán, S., Fraile, A., and García-Arenal, F. (2006). Association and host selectivity in multi-host pathogens. PLoS ONE 1, e41.

Marchler-Bauer, A., Lu, S., Anderson, J.B., Chitsaz, F., Derbyshire, M.K., DeWeeseScott, C., Fong, J.H., Geer, L.Y., Geer, R.C., and Gonzales, N.R. (2010). CDD: a Conserved Domain Database for the functional annotation of proteins. Nucleic Acids Research 39, D225-D229.

Marco, C.F., and Aranda, M.A. (2005). Genetic diversity of a natural population of Cucurbit yellow stunting disorder virus. Journal of General Virology 86, 815822.

Martin, D.P., Murrell, B., Golden, M., Khoosal, A., and Muhire, B. (2015). RDP4: Detection and analysis of recombination patterns in virus genomes. Virus Evolution 1, vev003.

Mathur, C., and Savithri, H.S. (2012). Novel ATPase activity of the polyprotein intermediate, Viral Protein genome-linked-Nuclear Inclusion-a protease, of Pepper vein banding potyvirus. Biochemical and Biophysical Research Communications 427, 113-118.

Mbanzibwa, D.R., Tian, Y.P., Tugume, A.K., Patil, B.L., Yadav, J.S., Bagewadi, B., Abarshi, M.M., Alicai, T., Changadeya, W., and Mkumbira, J. (2011). Evolution 
of cassava brown streak disease-associated viruses. Journal of General Virology 92, 974-987.

McCartney, H.A (1997). The influence of environment on the development and control of disease. In Environmentally Safe Approaches to Crop Disease Control (J. Rechcigl), pp. 3-31.

McGarigal, K., Cushman, S.A., and Ene, E. (2012). FRAGSTATS v4: spatial pattern analysis program for categorical and continuous maps. University of Massachusetts, Amherst, Massachusetts, USA. Goo. GI/AAEbMk.

McKenna, A., Hanna, M., Banks, E., Sivachenko, A., Cibulskis, K., Kernytsky, A., Garimella, K., Altshuler, D., Gabriel, S., Daly, M., et al. (2010). The Genome Analysis Toolkit: A MapReduce framework for analyzing next-generation DNA sequencing data. Genome Research 20, 1297-1303.

McLeish, M., Sacristán, S., Fraile, A., and García-Arenal, F. (2017). Scale dependencies and generalism in host use shape virus prevalence. Proceedings of the Royal Society B 284, 20172066.

Meier-Kolthoff, J.P., Auch, A.F., Huson, D.H., and Göker, M. (2007). CopyCat: cophylogenetic analysis tool. Bioinformatics 23, 898-900.

Melgarejo Nárdiz, P., García-Jiménez, J., Jordá Gutiérrez, M.C., López González, M.M., Andrés Yebes, M.F., and Duran-Vila, N. (2004). Patógenos de plantas descritos en España. Editorial Ministerio de Medio Ambiente y Medio Rural y Marino.

Mertler, C.A., and Reinhart, R.V. (2016). Advanced and multivariate statistical methods: Practical application and interpretation (Taylor \& Francis).

Mguis, K., Albouchi, A., and Brahim, N.B. (2011). Effect of temperature and salinity on germination of Ruta graveolens L. Acta Botanica Gallica 158, 645-652.

Miller, M.R., White, A., and Boots, M. (2007). Host life span and the evolution of resistance characteristics. Evolution 61, 2-14.

Mitchell, C.E., Reich, P.B., Tilman, D., and Groth, J.V. (2003). Effects of elevated CO2, nitrogen deposition, and decreased species diversity on foliar fungal plant disease. Global Change Biology 9, 438-451.

Mitchell, C.E., Tilman, D., and Groth, J.V. (2002). Effects of grassland plant species diversity, abundance, and composition on foliar fungal disease. Ecology 83, 1713-1726.

Mitchell, T.M. (1997). Machine learning. 1997. Burr Ridge, IL: McGraw Hill 45, 870877.

Mohasin, A.D., and Chona, N. (2002). Development of forewarning system of potato aphid (Myzus persicae) on potato (Solanum tuberosum) in India. Indian Journal of Agricultural Sciences 72, 341-5.

Mondal, H.A. (2017). Shaping the understanding of saliva-derived effectors towards aphid colony proliferation in host plant. Journal of Plant Biology 60, 103-115. 
Morales, F.J. (2006). History and current distribution of begomoviruses in Latin America. Advances in Virus Research 67, 127-162.

Morales, F.J., and Jones, P.G. (2004). The ecology and epidemiology of whiteflytransmitted viruses in Latin America. Virus Research 100, 57-65.

Moreno, A., Blas, C. de, Biurrun, R., Nebreda, M., Palacios, I., Duque, M., and Fereres, A. (2004a). The incidence and distribution of viruses infecting lettuce, cultivated Brassica and associated natural vegetation in Spain. Annals of Applied Biology 144, 339-346.

Moreno, I.M., Malpica, J.M., Díaz-Pendón, J.A., Moriones, E., Fraile, A., and GarcíaArenal, F. (2004b). Variability and genetic structure of the population of Watermelon mosaic virus infecting melon in Spain. Virology 318, 451-460.

Moreno-Pérez, M.G., Pagán, I., Aragón-Caballero, L., Cáceres, F., Fraile, A., and García-Arenal, F. (2014). Ecological and genetic determinants of Pepino mosaic virus emergence. Journal of Virology 88, 3359-3368.

Moriones, E., and Navas-Castillo, J. (2000). Tomato yellow leaf curl virus, an emerging virus complex causing epidemics worldwide. Virus Research 71, 123-134.

Moriones, E., and Navas-Castillo, J. (2008). Rapid evolution of the population of begomoviruses associated with the tomato yellow leaf curl disease after invasion of a new ecological niche: a review. Spanish Journal of Agricultural Research 6, 147-159.

Morse, S.S., and Schluederberg, A. (1990). Emerging viruses: the evolution of viruses and viral diseases. The Journal of Infectious Diseases 162, 1-7.

Moury, B., Fabre, F., and Senoussi, R. (2007). Estimation of the number of virus particles transmitted by an insect vector. Proceedings of the National Academy of Sciences 104, 17891-17896.

Moya, A., Elena, S.F., Bracho, A., Miralles, R., and Barrio, E. (2000). The evolution of RNA viruses: a population genetics view. Proceedings of the National Academy of Sciences 97, 6967-6973.

Moya, A., Holmes, E.C., and González-Candelas, F. (2004). The population genetics and evolutionary epidemiology of RNA viruses. Nature Reviews Microbiology 2, 279.

Moya, A., Rodriguez-Cerezo, E., and García-Arenal, F. (1993). Genetic structure of natural populations of the plant RNA virus Tobacco mild green mosaic virus. Molecular Biology and Evolution 10, 449-456

Murrell, B., Moola, S., Mabona, A., Weighill, T., Sheward, D., Kosakovsky Pond, S.L., and Scheffler, K. (2013). FUBAR: a fast, unconstrained bayesian approximation for inferring selection. Molecular Biology and Evolution 30, 1196-1205.

Nagelkerke, N.J. (1991). A note on a general definition of the coefficient of determination. Biometrika 78, 691-692. 
Navas-Castillo, J., Sánchez-Campos, S., Díaz, J.A., Sáez-Alonso, E., and Moriones, E. (1999). Tomato yellow leaf curl virus-Is causes a novel disease of common bean and severe epidemics in tomato in Spain. Plant Disease 83, 29-32.

Nebreda, M., Moreno, A., Pérez, N., Palacios, I., Seco-Fernández, V., and Fereres, A. (2004). Activity of aphids associated with lettuce and broccoli in Spain and their efficiency as vectors of Lettuce mosaic virus. Virus Research 100, 83-88.

Nei, M., and Tajima, F. (1981). Genetic drift and estimation of effective population size. Genetics 98, 625-640.

Ng, J.C., and Perry, K.L. (2004). Transmission of plant viruses by aphid vectors. Molecular Plant Pathology 5, 505-511.

Nicaise, V. (2014). Crop immunity against viruses: outcomes and future challenges. Frontiers in Plant Science 5, 660.

Oerke, E.-C. (2006). Crop losses to pests. The Journal of Agricultural Science 144, $31-43$.

Oerke, E.-C., and Dehne, H.-W. (2004). Safeguarding production-losses in major crops and the role of crop protection. Crop Protection 23, 275-285.

Ohshima, K., Yamaguchi, Y., Hirota, R., Hamamoto, T., Tomimura, K., Tan, Z., Sano, T., Azuhata, F., Walsh, J.A., and Fletcher, J. (2002). Molecular evolution of Turnip mosaic virus: evidence of host adaptation, genetic recombination and geographical spread. Journal of General Virology 83, 1511-1521.

Okonechnikov, K., Conesa, A., and García-Alcalde, F. (2015). Qualimap 2: advanced multi-sample quality control for high-throughput sequencing data. Bioinformatics 32, 292-294.

Ostfeld, R.S., and Keesing, F. (2012). Effects of host diversity on infectious disease. Annual Review of Ecology, Evolution, and Systematics 43, 157-182.

Pagán, I., Alonso-Blanco, C., and García-Arenal, F. (2007). The relationship of withinhost multiplication and virulence in a plant-virus system. PLoS ONE 2, e786.

Pagán, I., and García-Arenal, F. (2018). Population Genomics of Plant Viruses. In Population Genomics, (Springer), pp. 1-33.

Pagán, I., and Holmes, E.C. (2010). Long-term evolution of the Luteoviridae: time scale and mode of virus speciation. Journal of Virology 84, 6177-6187.

Pagán, I., Betancourt, M., de Miguel, J., Piñero, D., Fraile, A., and García-Arenal, F. (2010). Genomic and biological characterization of chiltepín yellow mosaic virus, a new tymovirus infecting Capsicum annuum var. aviculare in Mexico. Archives of Virology 155, 675-684.

Pagán, I., Fraile, A., and García-Arenal, F. (2016). Evolution of the interactions of viruses with their plant hosts. In Virus Evolution: Current Research and Future Directions, (Caister Academic Press), pp. 127-154.

Pagán, I., González-Jara, P., Moreno-Letelier, A., Rodelo-Urrego, M., Fraile, A., Piñero, D., and García-Arenal, F. (2012). Effect of biodiversity changes in 
disease risk: exploring disease emergence in a plant-virus system. PLOS Pathogens 8, e1002796.

Pagán, I., Montes, N., Milgroom, M.G., and García-Arenal, F. (2014). Vertical transmission selects for reduced virulence in a plant virus and for increased resistance in the host. PLoS Pathogens 10, e1004293.

Parker, I.M., Saunders, M., Bontrager, M., Weitz, A.P., Hendricks, R., Magarey, R., Suiter, K., and Gilbert, G.S. (2015). Phylogenetic structure and host abundance drive disease pressure in communities. Nature 520, 542.

Parker, J., Rambaut, A., and Pybus, O.G. (2008). Correlating viral phenotypes with phylogeny: accounting for phylogenetic uncertainty. Infection, Genetics and Evolution 8, 239-246.

Pasin, F., Simón-Mateo, C., and García, J.A. (2014). The hypervariable aminoterminus of $\mathrm{P} 1$ protease modulates potyviral replication and host defense responses. PLoS Pathogens 10, e1003985.

Patón, D., García-Herrera, R., Cuenca, J., Galavis, M., and Roig, F. (2010). Influence of climate on radial growth of holm oaks (Quercus Ilex subsp. Ballota Desf) from SW Spain. Geochronometria 34, 49-56.

Patz, J.A., Graczyk, T.K., Geller, N., and Vittor, A.Y. (2000). Effects of environmental change on emerging parasitic diseases. International Journal for Parasitology 30, 1395-1405.

Pfitzner, A.J.P. (2006). Resistance to Tobacco mosaic virus and Tomato mosaic virus in tomato. In Natural Resistance Mechanisms of Plants to Viruses, (Springer), pp. 399-413.

Pinel-Galzi, A., Traoré, O., Séré, Y., Hébrard, E., and Fargette, D. (2015). The biogeography of viral emergence: Rice yellow mottle virus as a case study. Current Opinion in Virology 10, 7-13.

Pita, J.S., Fondong, V.N., Sangare, A., Otim-Nape, G.W., Ogwal, S., and Fauquet, C.M. (2001). Recombination, pseudorecombination and synergism of geminiviruses are determinant keys to the epidemic of severe cassava mosaic disease in Uganda. Journal of General Virology 82, 655-665.

Pleydell, D.R., Soubeyrand, S., Dallot, S., Labonne, G., Chadoeuf, J., Jacquot, E., and Thebaud, G. (2018). Estimation of the dispersal distances of an aphid-borne virus in a patchy landscape. PLoS computational biology, 14, e1006085.

Posada, D. (2002). Evaluation of methods for detecting recombination from DNA sequences: empirical data. Molecular Biology and Evolution 19, 708-717.

Power, A.G. (1987). Plant community diversity, herbivore movement, and an insecttransmitted disease of maize. Ecology 68, 1658-1669.

Power, A.G., and Mitchell, C.E. (2004). Pathogen spillover in disease epidemics. The American Naturalist 164, S79-S89. 
Power, A.G., Borer, E.T., Hosseini, P., Mitchell, C.E., and Seabloom, E.W. (2011). The community ecology of barley/cereal yellow dwarf viruses in Western US grasslands. Virus Research 159, 95-100.

Prendeville, H.R., Tenhumberg, B., and Pilson, D. (2014). Effects of virus on plant fecundity and population dynamics. New Phytologist 202, 1346-1356.

Prendeville, H.R., Ye, X., Jack Morris, T., and Pilson, D. (2012). Virus infections in wild plant populations are both frequent and often unapparent. American Journal of Botany 99, 1033-1042.

Pressing, J., and Reanney, D.C. (1984). Divided genomes and intrinsic noise. Journal of Molecular Evolution 20,135-146.

Pruss, G., Ge, X., Shi, X.M., Carrington, J.C., and Vance, V.B. (1997). Plant viral synergism: the potyviral genome encodes a broad-range pathogenicity enhancer that transactivates replication of heterologous viruses. The Plant Cell 9, 859-868.

Pulido, F.J., Díaz, M., and de Trucios, S.J.H. (2001). Size structure and regeneration of Spanish holm oak Quercus ilex forests and dehesas: effects of agroforestry use on their long-term sustainability. Forest Ecology and Management 146, 113.

Pybus, O.G., and Rambaut, A. (2009). Evolutionary analysis of the dynamics of viral infectious disease. Nature Reviews Genetics 10, 540.

Quenouille, J., Vassilakos, N., and Moury, B. (2013). Potato virus Y: a major crop pathogen that has provided major insights into the evolution of viral pathogenicity. Molecular Plant Pathology 14, 439-452.

Quinlan, J.R. (1996). Improved use of continuous attributes in C4. 5. Journal of Artificial Intelligence Research 4, 77-90.

Rajamäki, M.-L., and Valkonen, J.P. (2009). Control of nuclear and nucleolar localization of nuclear inclusion protein a of picorna-like Potato virus $A$ in Nicotiana species. The Plant Cell 21, 2485-2502.

Rajamäki, M.-L., Kelloniemi, J., Alminaite, A., Kekarainen, T., Rabenstein, F., and Valkonen, J.P. (2005). A novel insertion site inside the potyvirus P1 cistron allows expression of heterologous proteins and suggests some P1 functions. Virology 342, 88-101.

Ramírez, J.A., and Díaz, M. (2008). The role of temporal shrub encroachment for the maintenance of Spanish holm oak Quercus ilex dehesas. Forest Ecology and Management 255, 1976-1983.

Ramsey, F.L., and Schafer, D.W. (2002). The statistical sleuth: A course in methods of data analysis. $2^{\text {nd }}$ Edition. Duxbury Press, Belmont, CA.

Randolph, S.E., and Dobson, A.D.M. (2012). Pangloss revisited: a critique of the dilution effect and the biodiversity-buffers-disease paradigm. Parasitology 139, 847-863. 
Restrepo-Hartwig, M.A., and Carrington, J.C. (1994). The tobacco etch potyvirus 6kilodalton protein is membrane associated and involved in viral replication. Journal of Virology 68, 2388-2397.

Revers, F., Le Gall, O., Candresse, T., and Maule, A.J. (1999). New advances in understanding the molecular biology of plant/potyvirus interactions. Molecular Plant-Microbe Interactions 12, 367-376.

Revers, F., Le Gall, O., Candresse, T., Le Romancer, M., and Dunez, J. (1996). Frequent occurrence of recombinant potyvirus isolates. Journal of General Virology 77, 1953-1965.

Reynaud, B., Delatte, H., Peterschmitt, M., and Fargette, D. (2009). Effects of temperature increase on the epidemiology of three major vector-borne viruses. European Journal of Plant Pathology 123, 269-280.

Riechmann, J.L., Cervera, M.T., and Garcia, J.A. (1995). Processing of the plum pox virus polyprotein at the P3-6K1 junction is not required for virus viability. Journal of General Virology 76, 951-956.

Riechmann, J.L., Lain, S., and García, J.A. (1992). Highlights and prospects of potyvirus molecular biology. Journal of General Virology 73, 1-16.

Rivas-Martínez, S. (2004). Global bioclimatics. Madrid, Spain.

Rivas-Martínez, S. (2007). Mapa de series, geoseries y geopermaseries de vegetación de España (Asociación Española de Fitosociología (AEFA)).

Rivas-Martínez, S., Penas, A., and Díaz, T.E. (2004). Biogeographic map of Europe. Cartographic Service, University of León.

Roche, B., and Guegan, J.-F. (2011). Ecosystem dynamics, biological diversity and emerging infectious diseases. Comptes Rendus Biologies 334, 385-392.

Rodà, F. (1999). Ecology of Mediterranean evergreen oak forests (Springer Science \& Business Media).

Rodelo-Urrego, M., García-Arenal, F., and Pagán, I. (2015). The effect of ecosystem biodiversity on virus genetic diversity depends on virus species: A study of chiltepin-infecting begomoviruses in Mexico. Virus Evolution 1, vev004.

Rodelo-Urrego, M., Pagán, I., González-Jara, P., Betancourt, M., Moreno-Letelier, A., Ayllón, M.A., Fraile, A., Piñero, D., and García-Arenal, F. (2013). Landscape heterogeneity shapes host-parasite interactions and results in apparent plantvirus codivergence. Molecular Ecology 22, 2325-2340.

Rodríguez-Nevado, C., Lam, T.T.-Y., Holmes, E.C., and Pagán, I. (2018). The impact of host genetic diversity on virus evolution and emergence. Ecology Letters 21, 253-263.

Rodríguez-Nevado, C., Montes, N., and Pagán, I. (2017). Ecological factors affecting infection risk and population genetic diversity of a novel potyvirus in its native wild ecosystem. Frontiers in Plant Science 8, 1958. 
Rojas Escoba, L.A. (1989). El análisis de suelos, plantas y aguas para riego (Bogotá: ICA).

Ronquist, F., Teslenko, M., Van Der Mark, P., Ayres, D.L., Darling, A., Höhna, S., Larget, B., Liu, L., Suchard, M.A., and Huelsenbeck, J.P. (2012). MrBayes 3.2: efficient Bayesian phylogenetic inference and model choice across a large model space. Systematic Biology 61, 539-542.

Roossinck, M.J. (2010). Lifestyles of plant viruses. Philosophical Transactions of the Royal Society B: Biological Sciences 365, 1899-1905.

Roossinck, M.J. (2011). The big unknown: plant virus biodiversity. Current Opinion in Virology 1, 63-67.

Roossinck, M.J. (2012). Plant virus metagenomics: biodiversity and ecology. Annual Review of Genetics 46, 359-369.

Roossinck, M.J. (2015). Plants, viruses and the environment: ecology and mutualism. Virology 479, 271-277.

Roossinck, M.J., and García-Arenal, F. (2015). Ecosystem simplification, biodiversity loss and plant virus emergence. Current Opinion in Virology 10, 56-62.

Roossinck, M.J., Martin, D.P., and Roumagnac, P. (2015). Plant virus metagenomics: Advances in virus discovery. Phytopathology 105, 716-727.

Roscher, C., Schumacher, J., Foitzik, O., and Schulze, E.-D. (2007). Resistance to rust fungi in Lolium perenne depends on within-species variation and performance of the host species in grasslands of different plant diversity. Oecologia 153, 173-183.

Rost, B., Yachdav, G., and Liu, J. (2004). The predictprotein server. Nucleic Acids Research 32, W321-W326.

Rúa, M.A., Pollina, E.C., Power, A.G., and Mitchell, C.E. (2011). The role of viruses in biological invasions: friend or foe? Current Opinion in Virology 1, 68-72.

Ruffalo, M., LaFramboise, T., and Koyutürk, M. (2011). Comparative analysis of algorithms for next-generation sequencing read alignment. Bioinformatics 27, 2790-2796.

Rybicki, E.P. (2015). A Top Ten list for economically important plant viruses. Archives of Virology 160, 17-20.

Sacristán, S., Fraile, A., and García-Arenal, F. (2004). Population dynamics of Cucumber mosaic virus in melon crops and in weeds in central Spain. Phytopathology 94, 992-998.

Sacristán, S., Malpica, J.M., Fraile, A., and García-Arenal, F. (2003). Estimation of population bottlenecks during systemic movement of Tobacco mosaic virus in tobacco plants. Journal of Virology 77, 9906-9911.

Salvaudon, L., and Shykoff, J.A. (2013). Variation in Arabidopsis developmental responses to oomycete infection: resilience vs changes in life history traits. New Phytologist 197, 919-926. 
Sánchez, F., Martínez-Herrera, D., Aguilar, I., and Ponz, F. (1998). Infectivity of turnip mosaic potyvirus cDNA clones and transcripts on the systemic host Arabidopsis thaliana and local lesion hosts. Virus Research 55, 207-219.

Sánchez, F., Sáez, M., Lunello, P., and Ponz, F. (2013). Plant viral elongated nanoparticles modified for log-increases of foreign peptide immunogenicity and specific antibody detection. Journal of Biotechnology 168, 409-415.

Sánchez, F., Wang, X., Jenner, C.E., Walsh, J.A., and Ponz, F. (2003). Strains of Turnip mosaic potyvirus as defined by the molecular analysis of the coat protein gene of the virus. Virus Research 94, 33-43.

Sanjuán, R., Agudelo-Romero, P., and Elena, S.F. (2009). Upper-limit mutation rate estimation for a plant RNA virus. Biology Letters 5, 394-396.

Sanjuan, R., Nebot, M.R., Chirico, N., Mansky, L.M., and Belshaw, R. (2010). Viral mutation rates. Journal of Virology 84, 9733-9748.

Savary, S., Teng, P.S., Willocquet, L., and Nutter Jr, F.W. (2006). Quantification and modeling of crop losses: a review of purposes. Annual Review of Phytopathology 44, 89-112.

Schaad, M.C., Jensen, P.E., and Carrington, J.C. (1997). Formation of plant RNA virus replication complexes on membranes: role of an endoplasmic reticulumtargeted viral protein. The EMBO Journal 16, 4049-4059.

Schenker, N., and Gentleman, J.F. (2001). On judging the significance of differences by examining the overlap between confidence intervals. The American Statistician 55, 182-186.

Schneider, C.A., Rasband, W.S., and Eliceiri, K.W. (2012). NIH Image to ImageJ: 25 years of image analysis. Nature Methods 9, 671-675.

Schneider, W.L., and Roossinck, M.J. (2001). Genetic diversity in RNA virus quasispecies is controlled by host-virus interactions. Journal of Virology 75, 6566-6571.

Scholle, S.O., Ypma, R.J., Lloyd, A.L., and Koelle, K. (2013). Viral substitution rate variation can arise from the interplay between within-host and epidemiological dynamics. The American Naturalist 182, 494-513.

Seabloom, E.W., Borer, E.T., Jolles, A., and Mitchell, C.E. (2009a). Direct and indirect effects of viral pathogens and the environment on invasive grass fecundity in Pacific Coast grasslands. Journal of Ecology 97, 1264-1273.

Seabloom, E.W., Borer, E.T., Lacroix, C., Mitchell, C.E., and Power, A.G. (2013). Richness and composition of niche-assembled viral pathogen communities. PLoS One 8, e55675.

Seabloom, E.W., Hosseini, P.R., Power, A.G., and Borer, E.T. (2009b). Diversity and composition of viral communities: coinfection of barley and cereal yellow dwarf viruses in California grasslands. The American Naturalist 173, E79-E98. 
Shannon, C.E. (1948). A mathematical theory of communication. The Bell System Technical Journal. 27, 623-656.

Shukla, D.D., Ward, C.W., and Brunt, A.A. (1994). The Potyviridae. (Cab International).

Sikora, E.J., Gudauskas, R.T., Murphy, J.F., Porch, D.W., Andrianifahanana, M., Zehnder, G.W., Bauske, E.M., Kemble, J.M., and Lester, D.F. (1998). A multivirus epidemic of tomatoes in Alabama. Plant Disease 82, 117-120.

Silva, S.J.C., Castillo-Urquiza, G.P., Hora-Júnior, B.T., Assunção, I.P., Lima, G.S.A., Pio-Ribeiro, G., Mizubuti, E.S.G., and Zerbini, F.M. (2012). Species diversity, phylogeny and genetic variability of begomovirus populations infecting leguminous weeds in northeastern Brazil. Plant Pathology 61, 457-467.

Simon-Loriere, E., and Holmes, E.C. (2011). Why do RNA viruses recombine? Nature Reviews Microbiology 9, 617.

Sobrepere, M., and Achon, M.A. (2001). Emergence of maize rough dwarf Fijivirus in Spain. In 9th Conf Virus Dis Gramineae Europe. York, UK.

Soitamo, A.J., Jada, B., and Lehto, K. (2011). HC-Pro silencing suppressor significantly alters the gene expression profile in tobacco leaves and flowers. BMC Plant Biology 11, 68.

Sorel, M., Garcia, J.A., and German-Retana, S. (2014). The Potyviridae cylindrical inclusion helicase: a key multipartner and multifunctional protein. Molecular Plant-Microbe Interactions 27, 215-226.

Spetz, C., and Valkonen, J.P. (2004). Potyviral 6K2 protein long-distance movement and symptom-induction functions are independent and host-specific. Molecular Plant-Microbe Interactions 17, 502-510.

Stella, J.C., Rodríguez-González, P.M., Dufour, S., and Bendix, J. (2013). Riparian vegetation research in Mediterranean-climate regions: common patterns, ecological processes, and considerations for management. Hydrobiologia 719, 291-315.

Stobbe, A.H., and Roossinck, M.J. (2014). Plant virus metagenomics: what we know and why we need to know more. Frontiers in Plant Science 5.

Streicker, D.G., Fenton, A., and Pedersen, A.B. (2013). Differential sources of host species heterogeneity influence the transmission and control of multihost parasites. Ecology Letters 16, 975-984.

Stukenbrock, E.H., and McDonald, B.A. (2008). The Origins of Plant Pathogens in Agro-Ecosystems. Annual Review of Phytopathology 46, 75-100.

Tamura, K., Stecher, G., Peterson, D., Filipski, A., and Kumar, S. (2013). MEGA6: molecular evolutionary genetics analysis version 6.0. Molecular Biology and Evolution 30, 2725-2729. 
Thackray, D.J., Diggle, A.J., Berlandier, F.A., and Jones, R.A. (2004). Forecasting aphid outbreaks and epidemics of Cucumber mosaic virus in lupin crops in a Mediterranean-type environment. Virus Research 100, 67-82.

Thorvaldsdóttir, H., Robinson, J.T., and Mesirov, J.P. (2013). Integrative Genomics Viewer (IGV): high-performance genomics data visualization and exploration. Briefings in Bioinformatics 14, 178-192.

Thrall, P.H., and Burdon, J.J. (1999). The spatial scale of pathogen dispersal: consequences for disease dynamics and persistence. Evolutionary Ecology Research 1,681-701.

Tromas, N., and Elena, S.F. (2010). The rate and spectrum of spontaneous mutations in a plant RNA virus. Genetics $185,983-989$.

Tromas, N., Zwart, M.P., Poulain, M., and Elena, S.F. (2014). Estimation of the in vivo recombination rate for a plant RNA virus. Journal of General Virology 95, 724732.

Tugume, A.K., Mukasa, S.B., and Valkonen, J.P.T. (2008). Natural wild hosts of Sweet potato feathery mottle virus show spatial differences in virus incidence and virus-like diseases in Uganda. Phytopathology 98, 640-652.

Turley, N.E., and Johnson, M.T. (2015). Ecological effects of aphid abundance, genotypic variation, and contemporary evolution on plants. Oecologia 178, 747-759.

Tuttle, J.R., Idris, A.M., Brown, J.K., Haigler, C.H., and Robertson, D. (2008). Geminivirus-mediated gene silencing from Cotton leaf crumple virus is enhanced by low temperature in cotton. Plant Physiology 148, 41-50.

Urcuqui-Inchima, S., Haenni, A.-L., and Bernardi, F. (2001). Potyvirus proteins: a wealth of functions. Virus Research 74, 157-175.

Valli, A., Gallo, A., Calvo, M., de Jesús Pérez, J., and García, J.A. (2014). A novel role of the potyviral helper component proteinase contributes to enhance the yield of viral particles. Journal of Virology 88, 9808-9818.

Valli, A., Lopez-Moya, J.J., and Garcia, J.A. (2007). Recombination and gene duplication in the evolutionary diversification of $\mathrm{P} 1$ proteins in the family Potyviridae. Journal of General Virology 88, 1016-1028.

Verchot, J., Herndon, K.L., and Carrington, J.C. (1992). Mutational analysis of the tobacco etch potyviral 35-kDa proteinase: identification of essential residues and requirements for autoproteolysis. Virology 190, 298-306.

Vijayan, V., López-González, S., Sánchez, F., Ponz, F., and Pagán, I. (2017). Virulence evolution of a sterilizing plant virus: Tuning multiplication and resource exploitation. Virus Evolution 3, vex033.

Virgós, E. (2001). Relative value of riparian woodlands in landscapes with different forest cover for medium-sized Iberian carnivores. Biodiversity \& Conservation 10, 1039-1049. 
Vurro, M., Bonciani, B., and Vannacci, G. (2010). Emerging infectious diseases of crop plants in developing countries: impact on agriculture and socio-economic consequences. Food Security 2, 113-132.

Walsh, J.A., and Jenner, C.E. (2002). Turnip mosaic virus and the quest for durable resistance. Molecular Plant Pathology 3, 289-300.

Waltermann, A., and Maiss, E. (2006). Detection of 6K1 as a mature protein of $6 \mathrm{kDa}$ in plum pox virus-infected Nicotiana benthamiana. Journal of General Virology 87, 2381-2386.

Ward Jr, J.H. (1963). Hierarchical grouping to optimize an objective function. Journal of the American Statistical Association 58, 236-244.

Way, M.J., and Heathcote, G.D. (1966). Interactions of crop density of field beans, abundance of Aphis fabae Scop., virus incidence and aphid control by chemicals. Annals of Applied Biology 57, 409-423.

Wei, T., and Wang, A. (2008). Biogenesis of cytoplasmic membranous vesicles for plant potyvirus replication occurs at endoplasmic reticulum exit sites in a COPIand COPII-dependent manner. Journal of Virology 82, 12252-12264.

Whitlock, M.C. (1996). The red queen beats the jack-of-all-trades: the limitations on the evolution of phenotypic plasticity and niche breadth. The American Naturalist 148, S65-S77.

Wilson, D.S. (1992). Complex interactions in metacommunities, with implications for biodiversity and higher levels of selection. Ecology 73, 1984-2000.

Witten, I.H., Frank, E., Hall, M.A., and Pal, C.J. (2016). Data Mining: Practical machine learning tools and techniques (Morgan Kaufmann).

Woolhouse, M.E. (2002). Population biology of emerging and re-emerging pathogens. Trends in Microbiology 10, s3-s7.

Woolhouse, M.E., Haydon, D.T., and Antia, R. (2005). Emerging pathogens: the epidemiology and evolution of species jumps. Trends in Ecology \& Evolution 20, 238-244.

Woolhouse, M.E., Taylor, L.H., and Haydon, D.T. (2001). Population biology of multihost pathogens. Science 292, 1109-1112.

Wu, X., Xu, Z., and Shaw, J.G. (1994). Uncoating of tobacco mosaic virus RNA in protoplasts. Virology 200, 256-262.

Wylie, S.J., and Jones, R.A.C. (2009). Role of recombination in the evolution of host specialization within Bean yellow mosaic virus. Phytopathology 99, 512-518.

Wylie, S.J., Kueh, J., Welsh, B., Smith, L.J., Jones, M.G.K., and Jones, R.A.C. (2002). A non-aphid-transmissible isolate of bean yellow mosaic potyvirus has an altered NAG motif in its coat protein. Archives of Virology 147, 1813-1820.

Xiong, R., and Wang, A. (2013). SCE1, the SUMO-conjugating enzyme in plants that interacts with Nlb, the RNA-dependent RNA polymerase of Turnip mosaic virus, is required for viral infection. Journal of Virology 87, 4704-4715. 
Yarden, G., Hemo, R., Livne, H., Maoz, E., Lev, E., Lecoq, H., and Raccah, B. (2000). Cross-protection of cucurbitaceae from zucchini yellow mosaic potyvirus. In VII Eucarpia Meeting on Cucurbit Genetics and Breeding 510, pp. 349-356.

Yasaka, R., Fukagawa, H., Ikematsu, M., Soda, H., Korkmaz, S., Golnaraghi, A., Katis, N., Ho, S.Y., Gibbs, A.J., and Ohshima, K. (2017). The timescale of emergence and spread of turnip mosaic potyvirus. Scientific Reports 7, 4240.

Yasaka, R., Nguyen, H.D., Ho, S.Y., Duchêne, S., Korkmaz, S., Katis, N., Takahashi, H., Gibbs, A.J., and Ohshima, K. (2014). The temporal evolution and global spread of Cauliflower mosaic virus, a plant pararetrovirus. PloS One 9, e85641.

Zhang, X., Zhang, X., Singh, J., Li, D., and Qu, F. (2012). Temperature-dependent survival of Turnip crinkle virus-infected arabidopsis plants relies on an RNA silencing-based defense that requires dcl2, AGO2, and HEN1. Journal of Virology 86, 6847-6854.

Zhao, J., Liu, Q., Zhang, H., Jia, Q., Hong, Y., and Liu, Y. (2012). RuBisCO small subunit is involved in tobamovirus movement and $\mathrm{Tm}^{2}$-mediated extreme resistance. Plant Physiology 161, 374-383.

Zheng, L., Rodoni, B.C., Gibbs, M.J., and Gibbs, A.J. (2010). A novel pair of universal primers for the detection of potyviruses. Plant Pathology 59, 211-220.

Zhou, C., and Zhou, Y. (2012). Strategies for viral cross protection in plants. In Antiviral Resistance in Plants, (Springer), pp. 69-81. 
APPENDIX 
Table A1.1. Ecological and climatic parameters recorded at each location and visit in riparian forests during 2013-2015.

\begin{tabular}{|c|c|c|c|c|c|c|c|}
\hline Location & Parameter & Summer 2013 & Autumn 2013 & Spring 2014 & Summer 2014 & Autumn 2014 & Spring 2015 \\
\hline \multirow{16}{*}{ El Escorial } & Plant density $(m)^{*}$ & $4.66 \pm 2.22$ & $6.03 \pm 4.89$ & $5.22 \pm 4.28$ & $5.08 \pm 3.55$ & $4.15 \pm 3.26$ & $4.13 \pm 3.28$ \\
\hline & Relative abundance $(\%)^{*}$ & $3.45 \pm 2.23$ & $3.57 \pm 3.57$ & $2.94 \pm 2.35$ & $3.23 \pm 2.49$ & $3.85 \pm 3.20$ & $3.13 \pm 2.78$ \\
\hline & Biomass $\left(\mathrm{m}^{3}\right)$ & 0.38 & - & 0.13 & 0.17 & 0.20 & 0.15 \\
\hline & $\mathrm{S}$ & 29 & 28 & 34 & 31 & 26 & 32 \\
\hline & $\mathrm{H}^{\prime}$ & 3.15 & 2.90 & 3.23 & 3.16 & 2.93 & 3.13 \\
\hline & $\mathrm{E}_{\mathrm{H}}$ & 0.94 & 0.87 & 0.92 & 0.92 & 0.90 & 0.90 \\
\hline & HPS & 15 & 13 & 15 & 18 & 8 & 16 \\
\hline & $\mathrm{T}_{\min }\left({ }^{\circ} \mathrm{C}\right)$ & 7.55 & 2.43 & 2.47 & 9.33 & 3.65 & 1.27 \\
\hline & $\mathrm{T}_{\text {med }}\left({ }^{\circ} \mathrm{C}\right)$ & 20.43 & 14.77 & 13.43 & 22.27 & 15.20 & 13.70 \\
\hline & $\mathrm{T}_{\max }\left({ }^{\circ} \mathrm{C}\right)$ & 32.95 & 27.07 & 27.07 & 34.27 & 29.65 & 27.53 \\
\hline & $\mathrm{RH}_{\min }(\%)$ & 13.50 & 19.00 & 18.67 & 13.67 & 30.00 & 16.33 \\
\hline & $\mathrm{RH}_{\text {med }}(\%)$ & 39.50 & 59.00 & 50.00 & 36.33 & 62.67 & 53.00 \\
\hline & $\mathrm{RH}_{\max }(\%)$ & 88.75 & 99.67 & 98.67 & 93.33 & 99.50 & 96.00 \\
\hline & Rainfalls (mm) & 159.00 & 434.67 & 431.00 & 211.33 & 594.50 & 230.67 \\
\hline & Soil pH & 5.50 & 5.50 & 5.50 & 5.50 & 5.50 & 5.50 \\
\hline & Soil moisture (\%)* & - & - & - & $5.04 \pm 1.77$ & $19.74 \pm 3.01$ & $12.11 \pm 9.17$ \\
\hline \multirow{16}{*}{ Palazuelos } & Plant density $(\mathrm{m})^{*}$ & $7.15 \pm 5.13$ & $7.35 \pm 9.17$ & $7.96 \pm 9.18$ & $13.06 \pm 13.36$ & $12.25 \pm 20.65$ & $10.21 \pm 22.30$ \\
\hline & Relative abundance $(\%)^{*}$ & $3.23 \pm 2.31$ & $3.13 \pm 3.82$ & $3.81 \pm 3.71$ & $4.35 \pm 3.97$ & $3.33 \pm 3.93$ & $4.76 \pm 5.47$ \\
\hline & Biomass $\left(m^{3}\right)$ & 0.08 & 0.01 & 0.08 & 0.04 & 0.03 & 0.07 \\
\hline & $\mathrm{S}$ & 31 & 32 & 26 & 23 & 30 & 21 \\
\hline & $\mathrm{H}^{\prime}$ & 3.17 & 2.92 & 2.82 & 2.76 & 2.83 & 2.50 \\
\hline & $\mathrm{E}_{\mathrm{H}}$ & 0.92 & 0.84 & 0.87 & 0.88 & 0.83 & 0.82 \\
\hline & HPS & 15 & 14 & 14 & 13 & 13 & 12 \\
\hline & $\mathrm{T}_{\min }\left({ }^{\circ} \mathrm{C}\right)$ & 5.58 & 0.07 & 0.97 & 6.63 & 4.50 & -0.73 \\
\hline & $T_{\text {med }}\left({ }^{\circ} \mathrm{C}\right)$ & 18.33 & 13.07 & 11.90 & 20.13 & 14.97 & 11.87 \\
\hline & $\mathrm{T}_{\max }\left({ }^{\circ} \mathrm{C}\right)$ & 31.10 & 25.93 & 24.27 & 32.57 & 27.50 & 24.70 \\
\hline & $\mathrm{RH}_{\min }(\%)$ & 14.25 & 17.00 & 15.67 & 12.00 & 24.67 & 15.33 \\
\hline & $\mathrm{RH}_{\text {med }}(\%)$ & 46.50 & 63.67 & 57.67 & 42.33 & 63.00 & 61.00 \\
\hline & $\mathrm{RH}_{\max }(\%)$ & 94.25 & 98.67 & 99.67 & 96.33 & 98.67 & 98.33 \\
\hline & Rainfalls (mm) & 216.00 & 277.33 & 349.00 & 138.67 & 507.00 & 232.00 \\
\hline & Soil pH & 5.50 & 5.50 & 5.50 & 5.50 & 5.50 & 5.50 \\
\hline & Soil moisture (\%)* & - & - & - & $6.95 \pm 0.47$ & $22.94 \pm 3.11$ & $9.99 \pm 0.62$ \\
\hline
\end{tabular}

*Mean \pm standard deviation for all plant species collected at each location and season.

S: Species richness; H': Shannon's index; $E_{H}$ : Shannon's equitability index; HPS: number of host plant species; $T_{\max }, T_{\min }, T_{\text {mean }}$ : Average of monthly maximum, minimum, and mean temperatures at a given season/location; $\mathrm{RH}_{\max }, \mathrm{RH}_{\min }, \mathrm{RH}_{\text {mean }}$ : Average of monthly maximum, minimum, and mean relative humidity at a given season/location; Rainfalls: Average of total rainfalls in a season; Soil moisture: Average of measures taken at eight quadrants of $1 \mathrm{~m}^{2}$ randomly selected in each location. 
Table A1.1. Continued.

\begin{tabular}{|c|c|c|c|c|c|c|c|}
\hline Location & Parameter & Summer 2013 & Autumn 2013 & Spring 2014 & Summer 2014 & Autumn 2014 & Spring 2015 \\
\hline \multirow{16}{*}{ Pollos } & Plant density $(m)^{*}$ & $4.83 \pm 5.77$ & $4.85 \pm 5.61$ & $3.27 \pm 2.92$ & $4.44 \pm 4.40$ & $4.39 \pm 4.11$ & $2.78 \pm 2.17$ \\
\hline & Relative abundance $(\%)^{*}$ & $3.13 \pm 2.52$ & $3.08 \pm 3.46$ & $2.65 \pm 3.05$ & $2.86 \pm 2.37$ & $3.23 \pm 3.32$ & $3.13 \pm 2.62$ \\
\hline & Biomass $\left(\mathrm{m}^{3}\right)$ & 0.11 & 0.10 & 0.13 & 0.13 & 0.06 & 0.14 \\
\hline & $\mathrm{S}$ & 32 & 32 & 35 & 35 & 31 & 32 \\
\hline & $\mathrm{H}^{\prime}$ & 3.15 & 2.97 & 3.09 & 3.26 & 2.98 & 3.14 \\
\hline & $\mathrm{E}_{\mathrm{H}}$ & 0.91 & 0.86 & 0.87 & 0.92 & 0.87 & 0.90 \\
\hline & HPS & 15 & 12 & 13 & 10 & 12 & 8 \\
\hline & $\mathrm{T}_{\min }\left({ }^{\circ} \mathrm{C}\right)$ & 6.60 & -0.80 & 1.23 & 7.33 & 3.83 & -0.73 \\
\hline & $\mathrm{T}_{\text {med }}\left({ }^{\circ} \mathrm{C}\right)$ & 18.68 & 12.90 & 12.67 & 20.47 & 15.40 & 12.57 \\
\hline & $\mathrm{T}_{\max }\left({ }^{\circ} \mathrm{C}\right)$ & 32.48 & 26.30 & 25.50 & 34.57 & 28.37 & 27.63 \\
\hline & $\mathrm{RH}_{\min }(\%)$ & 14.25 & 31.50 & 18.00 & 12.67 & 27.33 & 14.67 \\
\hline & $\mathrm{RH}_{\text {med }}(\%)$ & 49.00 & 65.50 & 56.33 & 44.33 & 67.33 & 60.50 \\
\hline & $\mathrm{RH}_{\max }(\%)$ & 96.25 & 99.00 & 99.00 & 93.33 & 98.00 & 97.00 \\
\hline & Rainfalls (mm) & 222.50 & 299.00 & 166.00 & 80.00 & 365.00 & 101.33 \\
\hline & Soil pH & 5.00 & 5.00 & 5.00 & 5.00 & 5.00 & 5.00 \\
\hline & Soil moisture (\%)* & - & - & - & - & - & $13.13 \pm 12.09$ \\
\hline \multirow{16}{*}{ Rascafría } & Plant density $(m)^{*}$ & $6.51 \pm 3.24$ & $4.94 \pm 4.20$ & $7.33 \pm 6.06$ & $4.53 \pm 4.00$ & $5.80 \pm 6.28$ & $3.85 \pm 4.15$ \\
\hline & Relative abundance $(\%)^{*}$ & $2.63 \pm 1.57$ & $3.48 \pm 3.47$ & $3.57 \pm 3.38$ & $2.63 \pm 2.25$ & $3.13 \pm 2.76$ & $2.78 \pm 3.64$ \\
\hline & Biomass $\left(m^{3}\right)$ & 0.49 & - & 0.18 & 0.19 & 0.04 & 0.24 \\
\hline & S & 38 & 28 & 28 & 38 & 32 & 36 \\
\hline & $\mathrm{H}^{\prime}$ & 3.47 & 3.00 & 2.95 & 3.30 & 3.12 & 3.01 \\
\hline & $\mathrm{E}_{\mathrm{H}}$ & 0.95 & 0.90 & 0.89 & 0.91 & 0.90 & 0.84 \\
\hline & HPS & 20 & 16 & 14 & 15 & 15 & 16 \\
\hline & $\mathrm{T}_{\min }\left({ }^{\circ} \mathrm{C}\right)$ & 2.38 & -1.45 & -0.90 & 2.37 & 1.40 & -3.80 \\
\hline & $\mathrm{T}_{\text {med }}\left({ }^{\circ} \mathrm{C}\right)$ & 15.33 & 13.80 & 9.50 & 16.87 & 14.15 & 9.70 \\
\hline & $\mathrm{T}_{\max }\left({ }^{\circ} \mathrm{C}\right)$ & 29.18 & 26.50 & 22.93 & 30.40 & 28.95 & 23.90 \\
\hline & $\mathrm{RH}_{\min }(\%)$ & 16.50 & 16.50 & 19.33 & 16.33 & 18.50 & 19.67 \\
\hline & $\mathrm{RH}_{\text {med }}(\%)$ & 50.50 & 64.00 & 61.00 & 47.67 & 69.00 & 62.00 \\
\hline & $\mathrm{RH}_{\max }(\%)$ & 97.25 & 97.00 & 97.67 & 96.33 & 98.50 & 97.00 \\
\hline & Rainfalls (mm) & 363.50 & 466.50 & 1334.67 & 216.00 & 814.50 & 392.67 \\
\hline & Soil pH & 5.50 & 5.50 & 5.50 & 5.50 & 5.50 & 5.50 \\
\hline & Soil moisture (\%)* & - & - & - & $3.75 \pm 1.25$ & $20.48 \pm 2.88$ & $8.01 \pm 1.13$ \\
\hline
\end{tabular}

S: Species richness; $H^{\prime}$ : Shannon's index; $E_{H}$ : Shannon's equitability index; HPS: number of host plant species; $T_{\max }, T_{\min }, T_{\text {mean }}$ : Average of monthly maximum, minimum, and mean temperatures at a given season/location; $\mathrm{RH}_{\max }, \mathrm{RH}_{\min }, \mathrm{RH}_{\text {mean }}$ : Average of monthly maximum, minimum, and mean relative humidity at a given season/location; Rainfalls: Average of total rainfalls in a season; Soil moisture: Average of measures taken at eight quadrants of $1 \mathrm{~m}^{2}$ randomly selected in each location. 
Table A1.1. Continued.

\begin{tabular}{|c|c|c|c|c|c|c|c|}
\hline Location & Parameter & Summer 2013 & Autumn 2013 & Spring 2014 & Summer 2014 & Autumn 2014 & Spring 2015 \\
\hline \multirow{16}{*}{ Santa Cruz de Retamar } & Plant density $(\mathrm{m})^{\star}$ & $4.31 \pm 2.17$ & $5.16 \pm 5.68$ & $3.24 \pm 2.37$ & $4.82 \pm 6.32$ & $4.10 \pm 4.32$ & $4.97 \pm 5.33$ \\
\hline & Relative abundance (\%)* & $3.45 \pm 2.32$ & $3.48 \pm 4.31$ & $3.33 \pm 3.53$ & $2.94 \pm 5.04$ & $3.70 \pm 3.88$ & $5.88 \pm 7.27$ \\
\hline & Biomass $\left(m^{3}\right)$ & 0.02 & 0.01 & 0.06 & 0.00 & 0.06 & 0.01 \\
\hline & $\mathrm{S}$ & 29 & 27 & 30 & 34 & 27 & 17 \\
\hline & $\mathrm{H}^{\prime}$ & 3.14 & 2.64 & 2.96 & 2.80 & 2.86 & 2.24 \\
\hline & $\mathrm{E}_{\mathrm{H}}$ & 0.93 & 0.80 & 0.87 & 0.79 & 0.87 & 0.79 \\
\hline & HPS & 16 & 8 & 10 & 13 & 12 & 7 \\
\hline & $\mathrm{T}_{\min }\left({ }^{\circ} \mathrm{C}\right)$ & 9.85 & 5.10 & 4.50 & 13.00 & 7.90 & 3.90 \\
\hline & $\mathrm{T}_{\text {med }}\left({ }^{\circ} \mathrm{C}\right)$ & 23.13 & 16.60 & 15.30 & 24.20 & 17.40 & 15.80 \\
\hline & $\mathrm{T}_{\max }\left({ }^{\circ} \mathrm{C}\right)$ & 35.30 & 27.37 & 27.97 & 36.13 & 29.90 & 29.60 \\
\hline & $\mathrm{RH}_{\min }(\%)$ & 14.00 & 23.00 & 17.00 & 14.00 & 23.00 & 17.00 \\
\hline & $\mathrm{RH}_{\text {med }}(\%)$ & 37.50 & 60.33 & 52.67 & 36.33 & 67.67 & 55.00 \\
\hline & $\mathrm{RH}_{\max }(\%)$ & 95.00 & 99.00 & 98.00 & 95.00 & 99.00 & 98.00 \\
\hline & Rainfalls (mm) & 107.00 & 419.33 & 315.33 & 45.33 & 1031.33 & 319.33 \\
\hline & Soil pH & 5.50 & 5.50 & 5.50 & 5.50 & 5.50 & 5.50 \\
\hline & Soil moisture $(\%)^{*}$ & - & - & - & $2.76 \pm 0.31$ & $9.80 \pm 2.59$ & $1.64 \pm 0.41$ \\
\hline
\end{tabular}

$\mathrm{S}$ : Species richness; H': Shannon's index; $E_{H}$ : Shannon's equitability index; HPS: number of host plant species; $T_{\max }, T_{\min }, T_{\text {mean }}$ : Average of monthly maximum, minimum, and mean temperatures at a given season/location; $\mathrm{RH}_{\max }, \mathrm{RH}_{\min }, \mathrm{RH}_{\text {mean }}$ : Average of monthly maximum, minimum, and mean relative humidity at a given season/location; Rainfalls: Average of total rainfalls in a season; Soil moisture: Average of measures taken at eight quadrants of $1 \mathrm{~m}^{2}$ randomly selected in each location. 
Table A1.2. Ecological and climatic parameters recorded at each location and visit in evergreen oak forests during 2013-2015.

\begin{tabular}{|c|c|c|c|c|c|c|c|}
\hline Location & Parameter & Summer 2013 & Autumn 2013 & Spring 2014 & Summer 2014 & Autumn 2014 & Spring 2015 \\
\hline \multirow{16}{*}{ Carbonero el Mayor } & Plant density $(m)^{*}$ & $3.51 \pm 1.91$ & $5.75 \pm 7.14$ & $4.95 \pm 3.57$ & $6.38 \pm 4.03$ & $4.91 \pm 3.85$ & $4.38 \pm 4.00$ \\
\hline & Relative abundance (\%) & $5.88 \pm 4.34$ & $5.56 \pm 7.77$ & $7.14 \pm 6.02$ & $6.25 \pm 8.76$ & $5.56 \pm 9.19$ & $5.88 \pm 8.84$ \\
\hline & Biomass $\left(m^{3}\right)$ & 0.09 & 0.03 & 0.02 & 0.01 & 0.02 & 0.02 \\
\hline & $\mathrm{S}$ & 18 & 18 & 14 & 16 & 18 & 17 \\
\hline & $\mathrm{H}^{\prime}$ & 2.55 & 2.19 & 2.31 & 2.12 & 2.05 & 2.05 \\
\hline & $\mathrm{E}_{\mathrm{H}}$ & 0.88 & 0.76 & 0.88 & 0.76 & 0.71 & 0.72 \\
\hline & HPS & 6 & 7 & 6 & 7 & 4 & 4 \\
\hline & $\mathrm{T}_{\min }\left({ }^{\circ} \mathrm{C}\right)$ & 4.27 & -0.70 & 0.97 & 6.57 & 3.00 & -1.20 \\
\hline & $T_{\text {med }}\left({ }^{\circ} \mathrm{C}\right)$ & 16.97 & 13.20 & 12.00 & 20.27 & 13.15 & 12.10 \\
\hline & $\mathrm{T}_{\max }\left({ }^{\circ} \mathrm{C}\right)$ & 31.17 & 25.50 & 24.60 & 33.23 & 25.55 & 26.13 \\
\hline & $\mathrm{RH}_{\min }(\%)$ & 14.67 & 24.00 & 18.33 & 14.33 & 29.50 & 17.67 \\
\hline & $\mathrm{RH}_{\text {med }}(\%)$ & 48.67 & 65.67 & 57.33 & 43.00 & 70.00 & 62.00 \\
\hline & $\mathrm{RH}_{\max }(\%)$ & 95.33 & 98.67 & 98.00 & 96.00 & 99.00 & 98.33 \\
\hline & Rainfalls (mm) & 96.00 & 660.00 & 244.00 & 156.00 & 674.00 & 190.67 \\
\hline & Soil pH & 5.50 & 5.50 & 5.50 & 5.50 & 5.50 & 5.50 \\
\hline & Soil moisture (\%)* & - & - & - & $2.60 \pm 0.47$ & $6.58 \pm 0.77$ & $3.59 \pm 0.46$ \\
\hline \multirow{16}{*}{ Cenicientos } & Plant density $(m)^{*}$ & $7.57 \pm 7.27$ & $6.14 \pm 4.32$ & $6.08 \pm 6.64$ & $4.75 \pm 6.01$ & $4.59 \pm 4.73$ & $5.15 \pm 3.34$ \\
\hline & Relative abundance $(\%)^{*}$ & $5.00 \pm 3.03$ & $3.98 \pm 4.53$ & $3.69 \pm 3.23$ & $4.55 \pm 6.86$ & $3.70 \pm 6.21$ & $4.55 \pm 4.26$ \\
\hline & Biomass $\left(\mathrm{m}^{3}\right)$ & 0.01 & 0.00 & - & 0.00 & 0.01 & 0.08 \\
\hline & S & 20 & 25 & 27 & 22 & 27 & 22 \\
\hline & $\mathrm{H}^{\prime}$ & 2.82 & 2.77 & 2.93 & 2.37 & 2.53 & 2.72 \\
\hline & $\mathrm{E}_{\mathrm{H}}$ & 0.94 & 0.86 & 0.89 & 0.77 & 0.77 & 0.88 \\
\hline & HPS & 7 & 7 & 8 & 7 & 4 & 7 \\
\hline & $\mathrm{T}_{\min }\left({ }^{\circ} \mathrm{C}\right)$ & 7.80 & 3.67 & 2.47 & 9.30 & 5.57 & 1.53 \\
\hline & $T_{\text {med }}\left({ }^{\circ} \mathrm{C}\right)$ & 19.43 & 13.97 & 12.17 & 20.90 & 14.60 & 12.53 \\
\hline & $\mathrm{T}_{\max }\left({ }^{\circ} \mathrm{C}\right)$ & 31.33 & 25.13 & 24.13 & 32.60 & 27.00 & 25.17 \\
\hline & $\mathrm{RH}_{\min }(\%)$ & 14.00 & 27.67 & 24.67 & 15.33 & 30.33 & 19.33 \\
\hline & $\mathrm{RH}_{\text {med }}(\%)$ & 44.00 & 66.00 & 61.00 & 41.33 & 75.00 & 62.00 \\
\hline & $\mathrm{RH}_{\max }(\%)$ & 94.00 & 100.00 & 100.00 & 98.33 & 100.00 & 100.00 \\
\hline & Rainfalls (mm) & 84.50 & 516.67 & 353.33 & 57.33 & 989.00 & 355.00 \\
\hline & Soil pH & 4.50 & 4.50 & 4.50 & 4.50 & 4.50 & 4.50 \\
\hline & Soil moisture $(\%)^{*}$ & - & - & - & $1.28 \pm 0.35$ & $8.79 \pm 1.41$ & $1.65 \pm 0.92$ \\
\hline
\end{tabular}

Mean \pm standard deviation for all plant species collected at each lor

S: Species richness; H': Shannon's index; $E_{H}$ : Shannon's equitability index; HPS: number of host plant species; $T_{\max }, T_{\min }, T_{\text {mean }}$ : Average of monthly maximum, minimum, and mean temperatures at moisture: Average of measures taken at eight quadrants of $1 \mathrm{~m}^{2}$ randomly selected in each location. 
Table A1.2. Continued.

\begin{tabular}{|c|c|c|c|c|c|c|c|}
\hline Location & Parameter & Summer 2013 & Autumn 2013 & Spring 2014 & Summer 2014 & Autumn 2014 & Spring 2015 \\
\hline \multirow{16}{*}{ EI Pardo } & Plant density $(m)^{*}$ & $4.29 \pm 2.90$ & $2.86 \pm 2.01$ & $5.77 \pm 5.37$ & $3.93 \pm 2.87$ & $3.78 \pm 4.33$ & $3.77 \pm 3.24$ \\
\hline & Relative abundance $(\%)^{*}$ & $4.17 \pm 2.77$ & $6.67 \pm 6.86$ & $3.85 \pm 3.70$ & $4.00 \pm 3.32$ & $5.56 \pm 7.28$ & $3.70 \pm 4.48$ \\
\hline & Biomass $\left(m^{3}\right)$ & 0.01 & 0.05 & 0.05 & 0.01 & 0.05 & 0.08 \\
\hline & $S$ & 24 & 15 & 26 & 25 & 18 & 27 \\
\hline & $\mathrm{H}^{\prime}$ & 2.95 & 2.26 & 2.86 & 2.89 & 2.25 & 2.74 \\
\hline & $\mathrm{E}_{\mathrm{H}}$ & 0.93 & 0.84 & 0.88 & 0.90 & 0.78 & 0.83 \\
\hline & HPS & 9 & 6 & 11 & 9 & 5 & 7 \\
\hline & $\mathrm{T}_{\min }\left({ }^{\circ} \mathrm{C}\right)$ & 8.80 & -1.45 & 2.87 & 11.33 & 5.93 & 0.70 \\
\hline & $\mathrm{T}_{\text {med }}\left({ }^{\circ} \mathrm{C}\right)$ & 22.37 & 12.70 & 14.50 & 23.70 & 16.93 & 12.45 \\
\hline & $\mathrm{T}_{\max }\left({ }^{\circ} \mathrm{C}\right)$ & 34.47 & 24.90 & 28.37 & 36.30 & 29.10 & 25.85 \\
\hline & $\mathrm{RH}_{\min }(\%)$ & 17.67 & 23.00 & 18.67 & 17.67 & 26.33 & 18.00 \\
\hline & $\mathrm{RH}_{\text {med }}(\%)$ & 38.75 & 52.67 & 46.33 & 37.00 & 56.67 & 48.50 \\
\hline & $\mathrm{RH}_{\max }(\%)$ & 85.67 & 90.00 & 89.00 & 86.33 & 89.33 & 88.50 \\
\hline & Rainfalls (mm) & 94.67 & 215.50 & 229.67 & 42.33 & 418.50 & 348.00 \\
\hline & Soil pH & 6.00 & 6.00 & 6.00 & 6.00 & 6.00 & 6.00 \\
\hline & Soil moisture $(\%)^{*}$ & - & - & - & - & $9.13 \pm 1.60$ & $3.58 \pm 0.71$ \\
\hline \multirow{16}{*}{ Marjaliza } & Plant density $(m)^{*}$ & $6.37 \pm 5.47$ & $5.38 \pm 4.51$ & $5.47 \pm 4.39$ & $2.79 \pm 2.06$ & $3.12 \pm 3.96$ & $5.89 \pm 5.11$ \\
\hline & Relative abundance $(\%)^{*}$ & $3.20 \pm 2.69$ & $5.26 \pm 5.99$ & $3.85 \pm 3.16$ & $5.56 \pm 7.32$ & $4.35 \pm 5.91$ & $4.17 \pm 4.58$ \\
\hline & Biomass $\left(m^{3}\right)$ & 0.25 & 0.08 & 0.02 & 0.00 & 0.02 & 0.11 \\
\hline & S & 31 & 19 & 26 & 18 & 23 & 24 \\
\hline & $\mathrm{H}^{\prime}$ & 3.14 & 2.45 & 2.96 & 2.26 & 2.42 & 2.71 \\
\hline & $\mathrm{E}_{\mathrm{H}}$ & 0.91 & 0.83 & 0.91 & 0.78 & 0.77 & 0.85 \\
\hline & HPS & 7 & 7 & 10 & 7 & 4 & 8 \\
\hline & $\mathrm{T}_{\min }\left({ }^{\circ} \mathrm{C}\right)$ & 9.95 & 3.90 & 3.80 & 12.37 & 6.90 & 2.30 \\
\hline & $\mathrm{T}_{\text {med }}\left({ }^{\circ} \mathrm{C}\right)$ & 21.93 & 15.83 & 14.87 & 24.17 & 17.17 & 12.50 \\
\hline & $\mathrm{T}_{\max }\left({ }^{\circ} \mathrm{C}\right)$ & 33.78 & 27.23 & 27.50 & 35.73 & 29.63 & 24.90 \\
\hline & $\mathrm{RH}_{\min }(\%)$ & 11.50 & 21.33 & 14.67 & 12.33 & 23.67 & 13.50 \\
\hline & $\mathrm{RH}_{\text {med }}(\%)$ & 39.00 & 59.67 & 48.00 & 33.67 & 62.00 & 54.50 \\
\hline & $\mathrm{RH}_{\max }(\%)$ & 89.00 & 99.00 & 93.00 & 86.00 & 97.33 & 98.50 \\
\hline & Rainfalls (mm) & 133.50 & 153.33 & 162.00 & 54.67 & 354.67 & 405.00 \\
\hline & Soil pH & 6.00 & 6.00 & 6.00 & 6.00 & 6.00 & 6.00 \\
\hline & Soil moisture (\%) ${ }^{*}$ & - & - & - & - & $3.36 \pm 1.06$ & $2.78 \pm 0.77$ \\
\hline
\end{tabular}

$\mathrm{S}$ : Species richness; $\mathrm{H}^{\prime}$ : Shannon's index; $\mathrm{E}_{\mathrm{H}}$ : Shannon's equitability index; HPS: number of host plant species; $T_{\max }, T_{\min }$, $T_{\text {mean }}$ : Average of monthly maximum, minimum, and mean temperatures at a given season/location; $\mathrm{RH}_{\max }, \mathrm{RH}_{\min }, \mathrm{RH}_{\text {mean }}$ : Average of monthly maximum, minimum, and mean relative humidity at a given season/location; Rainfalls: Average of total rainfalls in a season; Soil moisture: Average of measures taken at eight quadrants of $1 \mathrm{~m}^{2}$ randomly selected in each location. 
Table A1.2. Continued.

\begin{tabular}{|c|c|c|c|c|c|c|c|}
\hline Location & Parameter & Summer 2013 & Autumn 2013 & Spring 2014 & Summer 2014 & Autumn 2014 & Spring 2015 \\
\hline \multirow{16}{*}{ Montegancedo } & Plant density $(\mathrm{m})^{*}$ & $3.96 \pm 2.37$ & $4.98 \pm 3.01$ & $4.53 \pm 3.65$ & $4.89 \pm 5.73$ & $5.32 \pm 5.90$ & $4.31 \pm 3.03$ \\
\hline & Relative abundance $(\%)^{\star}$ & $4.17 \pm 2.69$ & $4.55 \pm 5.26$ & $4.21 \pm 3.80$ & $5.88 \pm 5.53$ & $6.67 \pm 6.40$ & $3.85 \pm 3.08$ \\
\hline & Biomass $\left(m^{3}\right)$ & 0.04 & 0.05 & 0.07 & 0.01 & 0.08 & 0.28 \\
\hline & $\mathrm{S}$ & 24 & 22 & 25 & 17 & 15 & 26 \\
\hline & $\mathrm{H}^{\prime}$ & 2.96 & 2.62 & 2.96 & 2.41 & 2.27 & 2.95 \\
\hline & $\mathrm{E}_{\mathrm{H}}$ & 0.93 & 0.85 & 0.92 & 0.85 & 0.84 & 0.90 \\
\hline & HPS & 7 & 6 & 7 & 6 & 2 & 6 \\
\hline & $\mathrm{T}_{\min }\left({ }^{\circ} \mathrm{C}\right)$ & 6.10 & 1.27 & 2.10 & 9.90 & 3.53 & 3.80 \\
\hline & $\mathrm{T}_{\text {med }}\left({ }^{\circ} \mathrm{C}\right)$ & 20.88 & 14.80 & 14.03 & 22.70 & 15.87 & 15.20 \\
\hline & $\mathrm{T}_{\max }\left({ }^{\circ} \mathrm{C}\right)$ & 33.83 & 27.20 & 27.93 & 35.50 & 29.70 & 29.33 \\
\hline & $\mathrm{RH}_{\min }(\%)$ & 11.25 & 20.67 & 17.00 & 12.33 & 24.00 & 13.00 \\
\hline & $\mathrm{RH}_{\text {med }}(\%)$ & 38.25 & 59.00 & 49.67 & 36.00 & 64.67 & 53.00 \\
\hline & $\mathrm{RH}_{\max }(\%)$ & 88.75 & 98.00 & 95.00 & 90.67 & 98.00 & 98.00 \\
\hline & Rainfalls (mm) & 74.00 & 366.67 & 307.33 & 56.00 & 582.00 & 239.33 \\
\hline & Soil pH & 6.00 & 6.00 & 6.00 & 6.00 & 6.00 & 6.00 \\
\hline & Soil moisture (\%)* & - & - & - & $1.66 \pm 0.39$ & $10.80 \pm 4.30$ & - \\
\hline
\end{tabular}

S: Species richness; H': Shannon's index; $E_{H}$ : Shannon's equitability index; HPS: number of host plant species; $T_{\max }, T_{\min }$, $T_{\text {mean }}$ : Average of monthly maximum, minimum, and mean temperatures at a given season/location; $\mathrm{RH}_{\max }, \mathrm{RH}_{\min }, \mathrm{RH}_{\text {mean }}$ : Average of monthly maximum, minimum, and mean relative humidity at a given season/location; Rainfalls: Average of total rainfalls in a season; Soil moisture: Average of measures taken at eight quadrants of $1 \mathrm{~m}^{2}$ randomly selected in each location. 
Table A1.3. Phylogenetic congruence between the phylogenies obtained with the Nlb and the CP sequences of potyvirus isolates.

\begin{tabular}{|c|c|c|c|}
\hline Virus name & Potyvirus isolate & GenBank Acc. number & P value* \\
\hline Arracacha mottle virus & AMoV_C-17 & NC_018176 & 0.001 \\
\hline Algerian watermelon mosaic virus & AWMV__H4 & EU4̄10442 & 0.001 \\
\hline \multirow[t]{2}{*}{ Basella rugose mosaic virus } & BaRMV_AC & DQ821938 & 0.825 \\
\hline & BaRMV_BR & DQ821939 & 0.798 \\
\hline \multirow[t]{2}{*}{ Banana bract mosaic virus } & BBrMV & DQ851496 & 0.001 \\
\hline & BBrMV_TRY & HM131454 & 0.001 \\
\hline \multirow[t]{5}{*}{ Bean common mosaic necrosis virus } & BCMNV̄_Michigan & NC_004047 & 0.001 \\
\hline & BCMNV_NL5 & HQ229993 & 0.001 \\
\hline & BCMNV_NL8 & HQ229994 & 0.001 \\
\hline & BCMNV_PV0413 & HG792063 & 0.001 \\
\hline & BCMNV_TN1 & HQ229995 & 0.001 \\
\hline \multirow[t]{11}{*}{ Bean common mosaic virus } & BCMV_CD031 & KM051430 & 0.001 \\
\hline & BCMV_CT & KM076650 & 0.001 \\
\hline & BCMV_DXH025 & KJ807812 & 0.001 \\
\hline & BCMV_HB & AB863630 & 0.001 \\
\hline & BCMV_MB & KC832502 & 0.001 \\
\hline & BCMV_MS1 & EU761198 & 0.001 \\
\hline & BCMV_NL1 & L15331 & 0.001 \\
\hline & BCMV_NL4 & L21766 & 0.001 \\
\hline & BCMV_PV0915 & HG792064 & 0.001 \\
\hline & BCMV_R & AJ312437 & 0.001 \\
\hline & BCMV_Y & AJ312438 & 0.001 \\
\hline \multirow[t]{5}{*}{ Bidens mottle virus } & BiMoV_B12 & EU250210 & 0.001 \\
\hline & BiMoV_B3 & EU250212 & 0.001 \\
\hline & BiMoV_B4 & EU250211 & 0.001 \\
\hline & BiMoV_HL & EU250214 & 0.001 \\
\hline & BiMoV_WF & EU250213 & 0.001 \\
\hline Brugmansia suaveolens mottle virus & BsMoV_Bs-Campinas & AB551370 & 0.001 \\
\hline \multirow{2}{*}{ Blue squill virus $A$} & BSVA_KP1 & JN052072 & 0.001 \\
\hline & BSVA_SW3 & JQ807999 & 0.001 \\
\hline \multirow[t]{3}{*}{ Beet mosaic virus } & BtMV_BtMV-Wa & AY206394 & 0.823 \\
\hline & BtMV_Inner Mongolia & DQ674263 & 0.793 \\
\hline & $\mathrm{BtMV}_{-}^{-}$Xinjiang & DQ674264 & 0.784 \\
\hline \multirow[t]{3}{*}{ Bean yellow mosaic virus } & BYMV__GB32A & HG970854 & 0.004 \\
\hline & BYMV_IbG & AB079887 & 0.003 \\
\hline & BYMV_M11 & AB079886 & 0.003 \\
\hline \multirow[t]{5}{*}{ Cowpea aphid-borne mosaic virus } & CABMV_BR1 & HQ880242 & 0.001 \\
\hline & CABMV_MG-Avr & HQ880243 & 0.001 \\
\hline & CABMV_RR3 & KM597165 & 0.001 \\
\hline & CABMV_RR4 & KM655833 & 0.001 \\
\hline & CABMV_Z & AF348210 & 0.001 \\
\hline \multirow[t]{5}{*}{ Canna yellow streak virus } & CaYSV & GQ421689 & 0.001 \\
\hline & CaYSV_BZ2 & KM882642 & 0.001 \\
\hline & CaYSV_NC1 & KM882644 & 0.001 \\
\hline & CaYSV_NC5 & KM882648 & 0.001 \\
\hline & CaYSV_OK & KM882640 & 0.001 \\
\hline Celery mosaic virus & CeMV_California & HQ676607 & 0.001 \\
\hline \multirow[t]{2}{*}{ Chilli ringspot virus } & ChiRSV-HN/14 & JN008909 & 0.001 \\
\hline & ChiRSV-HN/Yingzhou & JQ234922 & 0.001 \\
\hline \multirow[t]{4}{*}{ Chilli veinal mottle virus } & ChiVMV_Ca & AJ972878 & 0.001 \\
\hline & ChiVMV-Ch-Jal & GU170807 & 0.001 \\
\hline & ChiVMV_Wenchang & GQ98131 & 0.001 \\
\hline & ChiVMV_YN-tobacco & JX088636 & 0.001 \\
\hline \multirow[t]{3}{*}{ Clover yellow vein virus } & CIYVV_C̄YVV & HG970870 & 0.001 \\
\hline & CIYVV_Gm & KF975894 & 0.001 \\
\hline & CIYVV_No.30 & AB002698 & 0.001 \\
\hline \multirow[t]{2}{*}{ Cocksfoot streak virus } & CSV & AF499738 & 0.001 \\
\hline & CSV_CSV630wytham & EU119422 & 0.001 \\
\hline Daphne mosaic virus & DapM̄V & DQ299908 & 0.039 \\
\hline \multirow[t]{3}{*}{ Dasheen mosaic virus } & DMV_Caladium & AF048981 & 0.001 \\
\hline & DMV_M13 & AJ298033 & 0.001 \\
\hline & DMV_T10 & KJ786965 & 0.001 \\
\hline
\end{tabular}

${ }^{*} \mathrm{~A}$ total of $5(2 \%)$ non-significant and $266(98 \%)$ significant associations between phylogenies of the $\mathrm{Nlb}$ and the $\mathrm{CP}$ sequences of potyvirus isolates were retrieved in the cophylogenetic analysis using a significance threshold of $P=0.05$ (CopyCat v.2.03; Meier-Kolthoff et al., 2007). 
Table A1.3. Continued.

\begin{tabular}{|c|c|c|c|}
\hline Virus name & Potyvirus isolate & GenBank Acc. number & P value* \\
\hline \multirow[t]{5}{*}{ East asian passiflora virus } & EAPV_SY071 & AB690448 & 0.001 \\
\hline & EAPV_SY072 & AB690449 & 0.001 \\
\hline & EAPV_SY101 & AB690451 & 0.001 \\
\hline & EAPV_YM102 & AB690452 & 0.001 \\
\hline & EAPV_YW101 & AB690455 & 0.001 \\
\hline Fritillary virus $Y$ & FVY_Panan & AM039800 & 0.001 \\
\hline Habenaria mosaic virus & $\mathrm{HaMV} \mathrm{Ha}-1$ & AB818538 & 0.001 \\
\hline \multirow[t]{7}{*}{ Hardenbergia mosaic virus } & HarMV_57.1 & HQ161080 & 0.001 \\
\hline & HarMV_57.2 & HQ161081 & 0.001 \\
\hline & HarMV_MD2 & KJ152152 & 0.001 \\
\hline & HarMV_MD3 & KJ152153 & 0.001 \\
\hline & HarMV_MD4A & KJ152154 & 0.001 \\
\hline & HarMV_MD4C & KJ152156 & 0.001 \\
\hline & HarMV_MD4D & KJ152157 & 0.001 \\
\hline Henbane mosaic virus & HMV_PHYS/H & AM184113 & 0.001 \\
\hline \multirow[t]{5}{*}{ Japanese yam mosaic virus } & JYMV & AB027007 & 0.001 \\
\hline & JYMV_FX1 & KJ789141 & 0.002 \\
\hline & JYMV_SG1 & KJ789140 & 0.001 \\
\hline & JYMV_WX1 & KJ789138 & 0.002 \\
\hline & JYMV_WX3 & KJ789139 & 0.001 \\
\hline Konjak mosaic virus & KoMV_KoMV-F & AB219545 & 0.008 \\
\hline \multirow[t]{4}{*}{ Lily mottle virus } & LMoV_Bate5 & JN127341 & 0.001 \\
\hline & LMoV_ML61 & AB570195 & 0.002 \\
\hline & LMoV_Sb & AJ564636 & 0.002 \\
\hline & LMoV_SMi & AM048875 & 0.001 \\
\hline \multirow[t]{11}{*}{ Lettuce mosaic virus } & LMV_AF199 & KF268955 & 0.001 \\
\hline & LMV_Br6 & KJ161174 & 0.001 \\
\hline & LMV_CL574 & KJ161183 & 0.001 \\
\hline & LMV_ES16 & KJ161185 & 0.001 \\
\hline & LMV_FR25 & KJ161186 & 0.001 \\
\hline & LMV_Ham6 & KJ161187 & 0.001 \\
\hline & LMV_LMV-Cr & KF268956 & 0.001 \\
\hline & LMV_Muju & KF955619 & 0.001 \\
\hline & LMV_Tn517 & KJ161192 & 0.001 \\
\hline & LMV_Yar & KJ161194 & 0.001 \\
\hline & LMV_Yuhang & AJ306288 & 0.001 \\
\hline Lupine mosaic virus & LuMV_LU2 & EU847625 & 0.004 \\
\hline \multirow[t]{9}{*}{ Leek yellow stripe virus } & LYSV_1A3I & AB194623 & 0.001 \\
\hline & LYSV_3mEI7 & AB194621 & 0.001 \\
\hline & LYSV_AG1 & JX429967 & 0.001 \\
\hline & LYSV_Bate4 & JN127340 & 0.001 \\
\hline & LYSV_SG2 & JX429965 & 0.001 \\
\hline & LYSV_SW10 & KF597285 & 0.001 \\
\hline & LYSV_SW9 & KF597284 & 0.001 \\
\hline & LYSV_W-Ku & AB194622 & 0.001 \\
\hline & LYSV_Yuhan & AJ307057 & 0.001 \\
\hline \multirow[t]{2}{*}{ Maize dwarf mosaic virus } & MDMV̄_Arg & DQ973169 & 0.001 \\
\hline & MDMV_Italy & JX185302 & 0.001 \\
\hline Moroccan watermelon mosaic virus & MWMV_TN057 & EF579955 & 0.001 \\
\hline \multirow[t]{2}{*}{ Narcissus degeneration virus } & NDV_Marijiniup 2 & JQ395041 & 0.001 \\
\hline & NDV_Zhangzhou & AM182028 & 0.001 \\
\hline \multirow[t]{2}{*}{ Narcissus late season yellows virus } & NLSYV_Marijiniup8 & KC691259 & 0.001 \\
\hline & NLSYV_Zhangzhou & JQ326210 & 0.001 \\
\hline \multirow[t]{3}{*}{ Narcissus yellow stripe virus } & NYSV_Marijiniup 3 & JQ395042 & 0.001 \\
\hline & NYSV_Zhangzhou & EU430294 & 0.001 \\
\hline & NYSV_ZZ-2 & JQ911732 & 0.001 \\
\hline \multirow[t]{5}{*}{ Ornithogalum mosaic virus } & OrMV_Bate9 & JN127345 & 0.010 \\
\hline & OrMV_KP & JQ807997 & 0.014 \\
\hline & OrMV_OMV-O & D00615 & 0.018 \\
\hline & OrMV_SW3.1 & JQ807995 & 0.016 \\
\hline & OrMV_SW3.3 & JQ807996 & 0.012 \\
\hline
\end{tabular}

${ }^{*} \mathrm{~A}$ total of $5(2 \%)$ non-significant and 266 (98\%) significant associations between phylogenies of the $\mathrm{NIb}$ and the $\mathrm{CP}$ sequences of potyvirus isolates were retrieved in the cophylogenetic analysis using a significance threshold of $P=0.05$ (CopyCat v.2.03; Meier-Kolthoff et al., 2007). 
Table A1.3. Continued.

\begin{tabular}{|c|c|c|c|}
\hline Virus name & Potyvirus isolate & GenBank Acc. number & P value* \\
\hline \multirow[t]{8}{*}{ Onion yellow dwarf virus } & OYDV_13.L.Se & KF623538 & 0.001 \\
\hline & OYDV_2.T.Se & KF623539 & 0.001 \\
\hline & OYDV_5.L & KF623535 & 0.001 \\
\hline & OYDV_6.L.Se & KF623537 & 0.001 \\
\hline & OYDV_8.T & KF623536 & 0.001 \\
\hline & OYDV_O.70 & KF623541 & 0.001 \\
\hline & OYDV OYDV-Se & JX433019 & 0.001 \\
\hline & OYDV_Yuhang & AJ510223 & 0.001 \\
\hline Panax virus $Y$ & PanVY_2 & GQ916624 & 0.001 \\
\hline \multirow[t]{3}{*}{ Peanut mottle virus } & PeMoV_Australian & X73422 & 0.001 \\
\hline & PeMoV_Habin & KF977830 & 0.001 \\
\hline & PeMoV_M & AF023848 & 0.001 \\
\hline \multirow[t]{5}{*}{ Pennisetum mosaic virus } & PenMV_AZ1 & JX070145 & 0.001 \\
\hline & PenMV_AZGL2 & JX070143 & 0.001 \\
\hline & PenMV_B & AY642590 & 0.001 \\
\hline & PenMV_C & DQ977725 & 0.001 \\
\hline & PenMV_CD1 & JX070152 & 0.001 \\
\hline \multirow[t]{2}{*}{ Pepper mottle virus } & PepMoV_California & M96425 & 0.001 \\
\hline & PepMoV_Florida & AF501591 & 0.001 \\
\hline Pepper severe mosaic virus & PepSMV & AM181350 & 0.001 \\
\hline \multirow[t]{3}{*}{ Pokeweed mosaic virus } & PkMV_MD & JQ609096 & 0.001 \\
\hline & PkMV_MS-FR03 & JX291161 & 0.001 \\
\hline & PkMV_PA & JQ609095 & 0.001 \\
\hline \multirow[t]{4}{*}{ Papaya leaf-distortion mosaic virus } & PLDMV & NC_005028 & 0.001 \\
\hline & PLDMV_HaiNan-DF & JX974555 & 0.001 \\
\hline & PLDMV_P & AB088221 & 0.001 \\
\hline & PLDMV_Taiwan-CZ & JX416282 & 0.001 \\
\hline \multirow[t]{8}{*}{ Plum pox virus } & PPV_48-922 & AY912058 & 0.001 \\
\hline & PPV_El Amar & AM157175 & 0.001 \\
\hline & PPV_Fantasia & AY912056 & 0.001 \\
\hline & PPV_J4c & EU117116 & 0.001 \\
\hline & PPV_Penn12 & EF640939 & 0.001 \\
\hline & PPV_Penn5 & EF640933 & 0.001 \\
\hline & PPV_PS & AJ243957 & 0.001 \\
\hline & PPV_Vulcan & AY912057 & 0.001 \\
\hline \multirow[t]{6}{*}{ Papaya ringspot virus } & PRSV̄_E2 & KC345609 & 0.001 \\
\hline & PRSV_HNVb & KF791028 & 0.001 \\
\hline & PRSV_P-5-19 & EU882728 & 0.001 \\
\hline & PRSV_R3 & KJ746106 & 0.001 \\
\hline & PRSV_W & AY010722 & 0.001 \\
\hline & PRSV_W-1 & DQ374153 & 0.001 \\
\hline \multirow[t]{3}{*}{ Pea seed-borne mosaic virus } & PSbMV_DPD1 & NC_001671 & 0.003 \\
\hline & PSbMV_L1 & AJ252242 & 0.001 \\
\hline & PSbMV_NY & X89997 & 0.001 \\
\hline \multirow[t]{2}{*}{ Peanut stripe virus } & PStV_Blotch & U05771 & 0.001 \\
\hline & PStV_SN-Nib3 & AF200624 & 0.001 \\
\hline \multirow[t]{3}{*}{ Peru tomato mosaic virus } & PToMV_Cuzqueno2 & EU495235 & 0.001 \\
\hline & PToMV_PPK11 & AJ516010 & 0.001 \\
\hline & PToMV_PPK13 & NC_004573 & 0.001 \\
\hline \multirow[t]{2}{*}{ Potato virus $A$} & PVA_143-PVA & GU144321 & 0.001 \\
\hline & PVA_B11 & NC_004039 & 0.001 \\
\hline \multirow[t]{2}{*}{ Pepper veinal mottle virus } & PVMV_ns1 & FJ617225 & 0.001 \\
\hline & PVMV_P & AB126178 & 0.001 \\
\hline \multirow[t]{2}{*}{ Potato $V$ virus } & PVV_DV-42 & AJ243766 & 0.001 \\
\hline & PVV_KER.LAL.P & KC433411 & 0.001 \\
\hline \multirow[t]{6}{*}{ Potato virus $Y$} & PVY_Adgen & AJ890348 & 0.001 \\
\hline & PVY_Chile3 & FJ214726 & 0.001 \\
\hline & PVY_JVW-186 & KF770835 & 0.001 \\
\hline & PVY_LW & AJ890349 & 0.001 \\
\hline & PVY_HR1 & FJ204166 & 0.001 \\
\hline & PVY_Wilga MV99 & HE608963 & 0.001 \\
\hline Passion fruit woodiness virus & PWV_Gld-1 & AB761400 & 0.001 \\
\hline & PWV-MU & HQ122652 & 0.001 \\
\hline
\end{tabular}

${ }^{*} \mathrm{~A}$ total of $5(2 \%)$ non-significant and $266(98 \%)$ significant associations between phylogenies of the $\mathrm{Nlb}$ and the $\mathrm{CP}$ sequences of potyvirus isolates were retrieved in the cophylogenetic analysis using a significance threshold of $P=0.05$ (CopyCat v.2.03; Meier-Kolthoff et al., 2007). 
Table A1.3. Continued.

\begin{tabular}{|c|c|c|c|}
\hline Virus name & Potyvirus isolate & GenBank Acc. number & P value* \\
\hline \multirow{3}{*}{ Sugarcane mosaic virus } & SCMV & DQ141605 & 0.001 \\
\hline & SCMV_Ohio & JX188385 & 0.001 \\
\hline & SCMV_Seehausen & JX185303 & 0.001 \\
\hline \multirow[t]{2}{*}{ Soybean mosaic virus } & SMV_G7A & FJ640982 & 0.001 \\
\hline & SMV_P & AJ507388 & 0.001 \\
\hline \multirow[t]{3}{*}{ Sweet potato feathery mottle virus } & SPFM̄V_O-Arg & KF386013 & 0.001 \\
\hline & SPFMV_RC-Arg & KF386014 & 0.001 \\
\hline & SPFMV_S & NC_001841 & 0.001 \\
\hline \multirow[t]{3}{*}{ Sweet potato virus $C$} & SPVC_Arg & KF386015 & 0.001 \\
\hline & SPVC_C1 & GU207957 & 0.001 \\
\hline & SPVC_IL & JX489166 & 0.001 \\
\hline \multirow[t]{6}{*}{ Sweet potato virus $G$} & SPVG_GWB-G & JN613805 & 0.001 \\
\hline & SPVG_HG167 & KM014814 & 0.001 \\
\hline & SPVG_IS103 & KM014815 & 0.001 \\
\hline & SPVG_Jesus Maria & NC_018093 & 0.001 \\
\hline & SPVG_WT325 & KF790759 & 0.001 \\
\hline & SPVG_Z01001 & JN613806 & 0.001 \\
\hline \multirow[t]{4}{*}{ Sorghum mosaic virus } & SrMV_GX & KJ541740 & 0.001 \\
\hline & SrMV_H & U57358 & 0.001 \\
\hline & SrMV_Xiaoshan & NC_004035 & 0.001 \\
\hline & SrMV_Yuhang & AJ310̄0198 & 0.001 \\
\hline \multirow{2}{*}{ Sunflower chlorotic mottle virus } & SuCMoV_Chlorotic ringspots & GU181200 & 0.001 \\
\hline & SuCMoV_Common & GU181199 & 0.001 \\
\hline \multirow[t]{3}{*}{ Shallot yellow stripe virus } & SYSV_onion & AM267479 & 0.001 \\
\hline & SYSV_ZQ1 & AJ865077 & 0.001 \\
\hline & SYSV_ZQ2 & AJ865076 & 0.001 \\
\hline \multirow[t]{2}{*}{ Telosma mosaic virus } & TeMV_GX1 & KJ789129 & 0.001 \\
\hline & TeMV_Hanoi & DQ851493 & 0.001 \\
\hline \multirow[t]{5}{*}{ Tobacco etch virus } & TEV & M15239 & 0.001 \\
\hline & TEV_HAT-AU & KM282187 & 0.001 \\
\hline & TEV_Mex21 & KM282188 & 0.002 \\
\hline & TEV_N & KM282189 & 0.001 \\
\hline & TEV_TEV7DA & DQ986288 & 0.001 \\
\hline \multirow[t]{2}{*}{ Thunberg fritillary mosaic virus } & TFMV_Ningbo & HM584812 & 0.004 \\
\hline & TFMV_Panan-2 & AJ885005 & 0.001 \\
\hline \multirow[t]{2}{*}{ Tomato necrotic stunt virus } & ToNStV_MX5 & JX846918 & 0.001 \\
\hline & ToNStV_MX9354 & JQ314463 & 0.001 \\
\hline \multirow[t]{6}{*}{ Turnip mosaic virus } & TuMV_ŪK1 & AB194787 & 0.001 \\
\hline & TuMV_C1 & AF394601 & 0.001 \\
\hline & TuMV_CAR37 & DQ648592 & 0.001 \\
\hline & TuMV_CAR39 & EF374098 & 0.001 \\
\hline & TuMV_CAR51 & HQ637383 & 0.001 \\
\hline & TuMV_TW & AF394602 & 0.001 \\
\hline \multirow[t]{8}{*}{ Tobacco vein banding mosaic virus } & TVBMV_HN39 & EU734432 & 0.001 \\
\hline & TVBMV_JX & JN630471 & 0.001 \\
\hline & TVBMV_LW & JN630473 & 0.001 \\
\hline & TVBMV_PY & JN630469 & 0.001 \\
\hline & TVBMV_SDYS1 & HQ396791 & 0.001 \\
\hline & TVBMV_YN9.1 & KF444434 & 0.001 \\
\hline & TVBMV_YND & EF219408 & 0.001 \\
\hline & TVBMV_YY & JN630472 & 0.001 \\
\hline \multirow[t]{2}{*}{ Tobacco vein mottling virus } & TVMV & X04083 & 0.001 \\
\hline & TVMV_S & U38621 & 0.001 \\
\hline Verbena virus $Y$ & VVY_Michigan & EU564817 & 0.001 \\
\hline Watermelon mosaic virus & WMV_A08-160 & JF273465 & 0.001 \\
\hline & WMV_C05-463 & JF273458 & 0.001 \\
\hline & WMV_C06-257 & JF273463 & 0.001 \\
\hline & WMV_C07-349 & JF273461 & 0.001 \\
\hline & WMV_Cg09-640 & JF273467 & 0.001 \\
\hline & WMV_Fr & AY437609 & 0.001 \\
\hline Wild potato mosaic virus & WPMV & AJ437279 & 0.001 \\
\hline Wild tomato mosaic virus & WTMV_Laichau & DQ851495 & 0.001 \\
\hline & WTMV_XC-1 & KM114869 & 0.001 \\
\hline Wisteria vein mosaic virus & WVMV_Beijing & AY656816 & 0.001 \\
\hline
\end{tabular}

${ }^{*}$ A total of $5(2 \%)$ non-significant and $266(98 \%)$ significant associations between phylogenies of the $\mathrm{Nlb}$ and the $\mathrm{CP}$ sequences of potyvirus isolates were retrieved in the cophylogenetic analysis using a significance threshold of $P=0.05$ (CopyCat v.2.03; Meier-Kolthoff et al., 2007). 
Table A1.3. Continued.

\begin{tabular}{llll}
\hline Virus name & Potyvirus isolate & GenBank Acc. number & P value $^{*}$ \\
\hline Yam bean mosaic virus & YBMV_SR & JN190431 & 0.001 \\
Yam mild mosaic virus & YMMV_Brazil & JX470965 & 0.001 \\
& YMMV_GH6_NC_1 & KJ125473 & 0.001 \\
& YMMV_SSY_FX_1 & KJ125474 & 0.001 \\
& YMMV_TG_N_1 & KJ125475 & 0.001 \\
& YMMV_YB3_NC_1 & KJ125476 & 0.001 \\
Yam mosaic virus & YMMV_ZY_XZ_1 & KJ125479 & 0.001 \\
Zantedeschia mild mosaic virus & YMV_Ivory Coast & NC_004752 & 0.003 \\
Zucchini tigré mosaic virus & ZaMMV_TW & AY626825 & 0.001 \\
& ZTMV_E11045 & KC345608 & 0.001 \\
& ZTMV_Q10 & KC345605 & 0.001 \\
Zucchini yellow mosaic virus & ZTMV_Re01-25 & KC345607 & 0.001 \\
& ZTMV_VET-026 & KC345606 & 0.001 \\
& ZYMV_B & AY188994 & 0.001 \\
& ZYMV_KR-PA & AY278998 & 0.001 \\
& ZYMV_KR-PE & AY278999 & 0.001 \\
& ZYMV_KR-PS & AY279000 & 0.001 \\
& ZYMV_TW-TN3 & AF127929 & 0.001 \\
\hline
\end{tabular}

${ }^{*} \mathrm{~A}$ total of $5(2 \%)$ non-significant and $266(98 \%)$ significant associations between phylogenies of the NIb and the $\mathrm{CP}$ sequences of potyvirus isolates were retrieved in the cophylogenetic analysis using a significance threshold of $P=0.05$ (CopyCat v.2.03; Meier-Kolthoff et al., 2007). 
Table A1.4. List of potyvirus isolates obtained in this thesis (2013-2016).

\begin{tabular}{|c|c|c|c|c|c|c|}
\hline Isolate name & Acc № & Virus $^{1}$ & Sequenced & Location $^{2}$ & Host & Year \\
\hline CIYVV-ESC091_Tpr_PRI_2014 & Unpublished & CIYVV & $350-$ nt Nlb/CP & ESC & Trifolium pratense & 2014 \\
\hline CIYVV-ESC103_Tca_PRI_2015 & Unpublished & CIYVV & 350-nt Nlb/CP & ESC & Trifolium campestre & 2014 \\
\hline CIYVV-ESC131_Tpr_PRI_2015 & Unpublished & CIYVV & 350-nt Nlb & ESC & Trifolium pratense & 2014 \\
\hline CIYVV-PAL014_Tpr_PRI_2015 & Unpublished & CIYVV & 350-nt Nlb/CP & PAL & Trifolium pratense & 2015 \\
\hline CIYVV-PAL015_Tpr_PRI_2015 & Unpublished & CIYVV & 350-nt Nlb/CP & PAL & Trifolium pratense & 2015 \\
\hline CIYVV-PAL022_Tpr_PRI_2015 & Unpublished & CIYVV & 350-nt Nlb/CP & PAL & Trifolium pratense & 2015 \\
\hline CIYVV-PAL027_Tca_OTŌ_2013 & Unpublished & CIYVV & 350-nt Nlb/CP & PAL & Trifolium campestre & 2013 \\
\hline CIYVV-PAL030_Rpu_OTO_2013 & Unpublished & CIYVV & 350-nt Nlb/CP & PAL & Rumex pulcher & 2013 \\
\hline CIYVV-PAL033_Tpr_VER_2013 & Unpublished & CIYVV & 350-nt Nlb/CP & PAL & Trifolium pratense & 2013 \\
\hline CIYVV-PAL040_Tca_PRI_2015 & Unpublished & CIYVV & 350-nt Nlb & PAL & Trifolium campestre & 2015 \\
\hline CIYVV-PAL046_Tca_OTO__2013 & Unpublished & CIYVV & 350-nt Nlb/CP & PAL & Trifolium campestre & 2013 \\
\hline CIYVV-PAL061_Tpr_OTO_2013 & Unpublished & CIYVV & 350-nt Nlb/CP & PAL & Trifolium pratense & 2013 \\
\hline CIYVV-PAL078_RpūOTO_2013 & Unpublished & CIYVV & 350-nt Nlb/CP & PAL & Rumex pulcher & 2013 \\
\hline CIYVV-PAL092 Tpr PRI 2015 & Unpublished & CIYVV & 350-nt Nlb/CP & PAL & Trifolium pratense & 2015 \\
\hline ENMV-SCR099_Ara_PRI_2015 & Unpublished & ENMV & 350-nt Nlb & SCR & Andryala ragusina & 2015 \\
\hline ENMV-SCR177_Ara_VER_2013 & Unpublished & ENMV & 350-nt Nlb & SCR & Andryala ragusina & 2013 \\
\hline MeRV-PAR003_Rmo_PRI_2015 & Unpublished & MeRV & 350-nt Nlb & PAR & Ruta montana & 2015 \\
\hline MeRV-PAR005_Rmo_VER_2013 & MF953320 & MeRV & $\mathrm{CP}$ & PAR & Ruta montana & 2013 \\
\hline MeRV-PAR005_Rmo_VER_2013 & Unpublished & MeRV & 350-nt NIb & PAR & Ruta montana & 2013 \\
\hline MeRV-PAR006_Rmo_OTO_2013 & MF953306 & MeRV & $\mathrm{CP}$ & PAR & Ruta montana & 2013 \\
\hline MeRV-PAR006_Rmo_OTO_2013 & Unpublished & MeRV & 350-nt Nlb & PAR & Ruta montana & 2013 \\
\hline MeRV-PAR006_Rmo_PRI_2014 & MF953325 & MeRV & $\mathrm{CP}$ & PAR & Ruta montana & 2014 \\
\hline MeRV-PAR006_Rmo_PRI_2014 & Unpublished & MeRV & 350-nt Nlb & PAR & Ruta montana & 2014 \\
\hline MeRV-PAR007 Rmo VER 2013 & MF953319 & MeRV & $\mathrm{CP}$ & PAR & Ruta montana & 2013 \\
\hline MeRV-PAR007_Rmo_VER_2013 & Unpublished & MeRV & 350-nt Nlb & PAR & Ruta montana & 2013 \\
\hline MeRV-PAR008_Rmo_OTO_2015 & MF953350 & MeRV & $\mathrm{CP}$ & PAR & Ruta montana & 2015 \\
\hline MeRV-PAR008_Rmo_PRI_2015 & MF953343 & MeRV & $\mathrm{CP}$ & PAR & Ruta montana & 2015 \\
\hline MeRV-PAR008_Rmo_PRI_2015 & Unpublished & MeRV & 350-nt Nlb & PAR & Ruta montana & 2015 \\
\hline MeRV-PAR009_Rmo_OTO_2013 & Unpublished & MeRV & 350-nt Nlb & PAR & Ruta montana & 2013 \\
\hline MeRV-PAR009_Rmo_OTO_2015 & MF953355 & MeRV & $\mathrm{CP}$ & PAR & Ruta montana & 2015 \\
\hline MeRV-PAR009_Rmo_PRI_2014 & MF953323 & MeRV & $\mathrm{CP}$ & PAR & Ruta montana & 2014 \\
\hline MeRV-PAR011_Rmo_OTO_2015 & MF953351 & MeRV & $\mathrm{CP}$ & PAR & Ruta montana & 2015 \\
\hline MeRV-PAR012_Rmo_VER_2014 & MF953331 & MeRV & $\mathrm{CP}$ & PAR & Ruta montana & 2014 \\
\hline MeRV-PAR013_Rmo_PRI_2015 & MF953342 & MeRV & $\mathrm{CP}$ & PAR & Ruta montana & 2015 \\
\hline MeRV-PAR013_Rmo_PRI_2015 & Unpublished & MeRV & 350-nt NIb & PAR & Ruta montana & 2015 \\
\hline MeRV-PAR014_Rmo_OTO_2015 & MF953356 & MeRV & $\mathrm{CP}$ & PAR & Ruta montana & 2015 \\
\hline MeRV-PAR017_Rmo_PRI_2015 & Unpublished & MeRV & 350-nt Nlb & PAR & Ruta montana & 2015 \\
\hline MeRV-PAR024_Rmo_PRI_2015 & Unpublished & MeRV & 350-nt Nlb & PAR & Ruta montana & 2015 \\
\hline MeRV-PAR027_Rmo_PRI_2015 & MF953345 & MeRV & $\mathrm{CP}$ & PAR & Ruta montana & 2015 \\
\hline MeRV-PAR027_Rmo_PRI_2015 & Unpublished & MeRV & 350-nt Nlb & PAR & Ruta montana & 2015 \\
\hline MeRV-PAR027_Rmo_VER_2013 & MF953316 & MeRV & $\mathrm{CP}$ & PAR & Ruta montana & 2013 \\
\hline MeRV-PAR027_Rmo_VER_2013 & Unpublished & MeRV & 350-nt Nlb & PAR & Ruta montana & 2013 \\
\hline MeRV-PAR033_Rmo_PRI_2016 & MF953360 & MeRV & $\mathrm{CP}$ & PAR & Ruta montana & 2016 \\
\hline MeRV-PAR033_Rmo_VER_2013 & MF953314 & MeRV & $\mathrm{CP}$ & PAR & Ruta montana & 2013 \\
\hline MeRV-PAR034_Rmo_VER_2014 & MF953332 & MeRV & $\mathrm{CP}$ & PAR & Ruta montana & 2014 \\
\hline MeRV-PAR037_Rmo_PRI_2014 & MF953324 & MeRV & $\mathrm{CP}$ & PAR & Ruta montana & 2014 \\
\hline MeRV-PAR037_Rmo_PRI_2014 & Unpublished & MeRV & 350-nt Nlb & PAR & Ruta montana & 2014 \\
\hline MeRV-PAR037_Rmo_VER_2014 & MF953333 & MeRV & $\mathrm{CP}$ & PAR & Ruta montana & 2014 \\
\hline MeRV-PAR040_Rmo_OTO_2015 & MF953352 & MeRV & $\mathrm{CP}$ & PAR & Ruta montana & 2015 \\
\hline MeRV-PAR041_Rmo_PRI_2015 & Unpublished & MeRV & 350-nt Nlb & PAR & Ruta montana & 2015 \\
\hline MeRV-PAR042_Rmo_OTO_2015 & MF953353 & MeRV & $\mathrm{CP}$ & PAR & Ruta montana & 2015 \\
\hline MeRV-PAR043_Rmo_OTO_2013 & MF953307 & MeRV & $\mathrm{CP}$ & PAR & Ruta montana & 2013 \\
\hline MeRV-PAR043_Rmo_OTO_2013 & Unpublished & MeRV & 350-nt Nlb & PAR & Ruta montana & 2013 \\
\hline MeRV-PAR046_Rmo_VER_2013 & MF953318 & MeRV & $\mathrm{CP}$ & PAR & Ruta montana & 2013 \\
\hline MeRV-PAR046_Rmo_VER_2013 & Unpublished & MeRV & 350-nt Nlb & PAR & Ruta montana & 2013 \\
\hline MeRV-PAR047_Rmo_OTO_2015 & MF953354 & MeRV & $\mathrm{CP}$ & PAR & Ruta montana & 2015 \\
\hline MeRV-PAR060_Rmo_PRI_2016 & MF953361 & MeRV & $\mathrm{CP}$ & PAR & Ruta montana & 2016 \\
\hline MeRV-PAR060_Rmo_VER_2014 & MF953334 & MeRV & $\mathrm{CP}$ & PAR & Ruta montana & 2014 \\
\hline MeRV-PAR061_Rmo_PRI_2016 & MF953362 & MeRV & $\mathrm{CP}$ & PAR & Ruta montana & 2016 \\
\hline MeRV-PAR061_Rmo_VER_2015 & MF953347 & MeRV & $\mathrm{CP}$ & PAR & Ruta montana & 2015 \\
\hline MeRV-PAR063_Rmo_PRI_2014 & MF953322 & MeRV & $\mathrm{CP}$ & PAR & Ruta montana & 2014 \\
\hline MeRV-PAR063_Rmo_PRI_2014 & Unpublished & MeRV & 350-nt Nlb & PAR & Ruta montana & 2014 \\
\hline MeRV-PAR063_Rmo_PRI_2016 & MF953363 & MeRV & $\mathrm{CP}$ & PAR & Ruta montana & 2016 \\
\hline MeRV-PAR064_Rmo_OTO_2013 & MF953308 & MeRV & $\mathrm{CP}$ & PAR & Ruta montana & 2013 \\
\hline MeRV-PAR064_Rmo_OTO_2013 & Unpublished & MeRV & 350-nt Nlb & PAR & Ruta montana & 2013 \\
\hline MeRV-PAR065_Rmo_OTO_2013 & MF953309 & MeRV & $\mathrm{CP}$ & PAR & Ruta montana & 2013 \\
\hline MeRV-PAR065_Rmo_OTO_2013 & Unpublished & MeRV & 350-nt Nlb & PAR & Ruta montana & 2013 \\
\hline MeRV-PAR065_Rmo_PRI_2016 & MF953364 & MeRV & $\mathrm{CP}$ & PAR & Ruta montana & 2016 \\
\hline
\end{tabular}

1Virus name: CIYVV: Clover yellow vein virus; ENMV: Endive necrotic mosaic virus; MeRV: Mediterranean ruda virus; WHMV: Wild hop mosaic virus

'Locations are designed with a three-letter code: ESC, El Escorial; MAR: Marjaliza; PAL: Palazuelos; PAR: EI Pardo; POL, Pollos; SCR, Santa Cruz de Retamar. 
Table A1.4. Continued.

\begin{tabular}{|c|c|c|c|c|c|c|}
\hline Isolate name & Acc № & Virus $^{1}$ & Sequenced & Location $^{2}$ & Host & Year \\
\hline MeRV-PAR066_Rmo_OTO_2013 & MF953310 & MeRV & $\mathrm{CP}$ & PAR & Ruta montana & 2013 \\
\hline MeRV-PAR066_Rmo_OTO_2013 & Unpublished & MeRV & 350-nt NIb & PAR & Ruta montana & 2013 \\
\hline MeRV-PAR066_Rmo_OTO_2015 & MF953357 & MeRV & $\mathrm{CP}$ & PAR & Ruta montana & 2015 \\
\hline MeRV-PAR067_Rmo_OTO_2013 & Unpublished & MeRV & 350-nt Nlb & PAR & Ruta montana & 2013 \\
\hline MeRV-PAR068_Rmo_OTO_2015 & MF953358 & MeRV & $\mathrm{CP}$ & PAR & Ruta montana & 2015 \\
\hline MeRV-PAR068 Rmo VER 2015 & MF953349 & MeRV & $\mathrm{CP}$ & PAR & Ruta montana & 2015 \\
\hline MeRV-PAR069_Rmo_OTO_2013 & MF953311 & MeRV & $\mathrm{CP}$ & PAR & Ruta montana & 2013 \\
\hline MeRV-PAR069_Rmo_OTO_2013 & Unpublished & MeRV & 350-nt NIb & PAR & Ruta montana & 2013 \\
\hline MeRV-PAR076 Rmo PRI 2016 & MF953365 & MeRV & $\mathrm{CP}$ & PAR & Ruta montana & 2016 \\
\hline MeRV-PAR081_Rmo_VER_2014 & MF953327 & MeRV & $\mathrm{CP}$ & PAR & Ruta montana & 2014 \\
\hline MeRV-PAR084 Rmo PRI 2016 & MF953366 & MeRV & $\mathrm{CP}$ & PAR & Ruta montana & 2016 \\
\hline MeRV-PAR087_RmO_OTŌ2015 & MF953359 & MeRV & $\mathrm{CP}$ & PAR & Ruta montana & 2015 \\
\hline MeRV-PAR088_Rmo_VER_2013 & MF953317 & MeRV & $\mathrm{CP}$ & PAR & Ruta montana & 2013 \\
\hline MeRV-PAR088_Rmo_VER_2013 & Unpublished & MeRV & 350-nt Nlb & PAR & Ruta montana & 2013 \\
\hline MeRV-PAR089_Rmo_OTO_2013 & MF953312 & MeRV & $\mathrm{CP}$ & PAR & Ruta montana & 2013 \\
\hline MeRV-PAR089 Rmo OTO 2013 & Unpublished & MeRV & 350-nt NIb & PAR & Ruta montana & 2013 \\
\hline MeRV-PAR089_Rmo_PRI_2016 & MF953367 & MeRV & $\mathrm{CP}$ & PAR & Ruta montana & 2016 \\
\hline MeRV-PAR089_Rmo_VER_2015 & MF953346 & MeRV & $\mathrm{CP}$ & PAR & Ruta montana & 2015 \\
\hline MeRV-PAR090 Rmo PRI 2014 & MF953321 & MeRV & $\mathrm{CP}$ & PAR & Ruta montana & 2014 \\
\hline MeRV-PAR090_Rmo_PRI_2014 & Unpublished & MeRV & 350-nt NIb & PAR & Ruta montana & 2014 \\
\hline MeRV-PAR096 Rmo PRI 2016 & MF953368 & MeRV & $\mathrm{CP}$ & PAR & Ruta montana & 2016 \\
\hline MeRV-PAR097_Rmo_PRI_2016 & MF953369 & MeRV & $\mathrm{CP}$ & PAR & Ruta montana & 2016 \\
\hline MeRV-PAR111_Rmo_PRI_2016 & MF953370 & MeRV & $\mathrm{CP}$ & PAR & Ruta montana & 2016 \\
\hline MeRV-PAR113_Rmo_OTO_2014 & MF953335 & MeRV & $\mathrm{CP}$ & PAR & Ruta montana & 2014 \\
\hline MeRV-PAR114_Rmo_OTO_2013 & MF953313 & MeRV & $\mathrm{CP}$ & PAR & Ruta montana & 2013 \\
\hline MeRV-PAR114 Rmo OTO 2013 & Unpublished & MeRV & 350-nt Nlb & PAR & Ruta montana & 2013 \\
\hline MeRV-PAR115_Rmo_OTO_2014 & MF953336 & MeRV & $\mathrm{CP}$ & PAR & Ruta montana & 2014 \\
\hline MeRV-PAR116 Rmo OTO 2014 & MF953337 & MeRV & $\mathrm{CP}$ & PAR & Ruta montana & 2014 \\
\hline MeRV-PAR118_Rmo_VER_2014 & MF953326 & MeRV & $\mathrm{CP}$ & PAR & Ruta montana & 2014 \\
\hline MeRV-PAR119_Rmo_OTO_2014 & MF953338 & MeRV & $\mathrm{CP}$ & PAR & Ruta montana & 2014 \\
\hline MeRV-PAR120_Rmo_OTO_2014 & MF953339 & MeRV & $\mathrm{CP}$ & PAR & Ruta montana & 2014 \\
\hline MeRV-PAR122_Rmo_PRI_2016 & MF953371 & MeRV & $\mathrm{CP}$ & PAR & Ruta montana & 2016 \\
\hline MeRV-PAR124_Rmo_PRI_2016 & MF953372 & MeRV & $\mathrm{CP}$ & PAR & Ruta montana & 2016 \\
\hline MeRV-PAR125_Rmo_PRI_2016 & MF953373 & MeRV & $\mathrm{CP}$ & PAR & Ruta montana & 2016 \\
\hline MeRV-PAR130_Rmo_VER_2015 & MF953348 & MeRV & $\mathrm{CP}$ & PAR & Ruta montana & 2015 \\
\hline MeRV-PAR135_Rmo_VER_2014 & MF953328 & MeRV & $\mathrm{CP}$ & PAR & Ruta montana & 2014 \\
\hline MeRV-PAR136_Rmo_VER_2014 & MF953330 & MeRV & $\mathrm{CP}$ & PAR & Ruta montana & 2014 \\
\hline MeRV-PAR137 Rmo VER 2014 & MF953329 & MeRV & $\mathrm{CP}$ & PAR & Ruta montana & 2014 \\
\hline MeRV-PAR140_Rmo_OTO_2014 & MF953340 & MeRV & $\mathrm{CP}$ & PAR & Ruta montana & 2014 \\
\hline MeRV-PAR142_Rmo_OTO_2014 & MF953341 & MeRV & $\mathrm{CP}$ & PAR & Ruta montana & 2014 \\
\hline MeRV-PAR166_Rmo_PRI_2016 & MF953374 & MeRV & $\mathrm{CP}$ & PAR & Ruta montana & 2016 \\
\hline MeRV-PAR183_Rmo_VER_2013 & MF953315 & MeRV & $\mathrm{CP}$ & PAR & Ruta montana & 2013 \\
\hline MeRV-PAR183 Rmo VER 2013 & Unpublished & MeRV & 350-nt NIb & PAR & Ruta montana & 2013 \\
\hline MeRV-ParP17 & MF953344 & MeRV & Full genome & PAR & Ruta montana & 2015 \\
\hline WHMV-ESC004_Tpr_PRI_2016 & Unpublished & WHMV & $350-n t ~ N I b ~$ & ESC & Trifolium pratense & 2016 \\
\hline WHMV-ESC031_Tpr_PRI_2016 & Unpublished & WHMV & 350-nt Nlb & ESC & Trifolium pratense & 2016 \\
\hline WHMV-ESC037_Tpr_PRI_2015 & Unpublished & WHMV & 350-nt NIb & ESC & Trifolium pratense & 2015 \\
\hline WHMV-ESC042_Tpr_PRI_2015 & Unpublished & WHMV & 350-nt NIb & ESC & Trifolium pratense & 2015 \\
\hline WHMV-ESC057_Tpr_PRI_2015 & Unpublished & WHMV & 350-nt Nlb & ESC & Trifolium pratense & 2015 \\
\hline WHMV-ESC081_Tpr_OTO_2014 & Unpublished & WHMV & 350-nt NIb & ESC & Trifolium pratense & 2014 \\
\hline WHMV-ESC094_Tpr_VER_2015 & Unpublished & WHMV & 350-nt Nlb & ESC & Trifolium pratense & 2015 \\
\hline WHMV-ESC101_Tpr_PRI_2015 & Unpublished & WHMV & 350-nt NIb & ESC & Trifolium pratense & 2015 \\
\hline WHMV-ESC107_Tpr_PRI_2015 & Unpublished & WHMV & 350-nt Nlb & ESC & Trifolium pratense & 2015 \\
\hline WHMV-ESC109_Tpr_PRI_2016 & Unpublished & WHMV & 350-nt Nlb & ESC & Trifolium pratense & 2016 \\
\hline WHMV-ESC127_Tpr_OTO_2014 & Unpublished & WHMV & 350-nt NIb & ESC & Trifolium pratense & 2014 \\
\hline WHMV-MAR043_Cal_PRI_2014 & Unpublished & WHMV & 350-nt Nlb & MAR & Cistus albidus & 2014 \\
\hline WHMV-PAL025_Tpr_PRI_2015 & Unpublished & WHMV & 350-nt NIb & PAL & Trifolium pratense & 2015 \\
\hline WHMV-PAL039_Tca_PRI_2015 & Unpublished & WHMV & 350-nt Nlb & PAL & Trifolium campestre & 2015 \\
\hline WHMV-PAL040_Tca_PRI_2015 & Unpublished & WHMV & 350-nt Nlb & PAL & Trifolium campestre & 2015 \\
\hline WHMV-PAL057 Tca PRI 2015 & Unpublished & WHMV & 350-nt NIb & PAL & Trifolium campestre & 2015 \\
\hline WHMV-PAL059_Tpr_PRI_2015 & Unpublished & WHMV & 350-nt Nlb & PAL & Trifolium pratense & 2015 \\
\hline WHMV-POL002_Hlu_PRI_2015 & Unpublished & WHMV & 350-nt Nlb/CP & POL & Humulus lupulus & 2015 \\
\hline WHMV-POL004_Hlu_PRI_2015 & Unpublished & WHMV & 350-nt Nlb & POL & Humulus lupulus & 2015 \\
\hline WHMV-POL005_Hlu_PRI_2015 & Unpublished & WHMV & 350-nt NIb & POL & Humulus lupulus & 2015 \\
\hline WHMV-POL008 Hlu PRI 2015 & Unpublished & WHMV & 350-nt Nlb/CP & POL & Humulus lupulus & 2015 \\
\hline WHMV-POL009_Eel_PRI_2014 & Unpublished & WHMV & 350-nt Nlb & POL & Ecballium elaterium & 2014 \\
\hline WHMV-POL009_HIU_VER_2014 & Unpublished & WHMV & 350-nt NIb & POL & Humulus Iupulus & 2014 \\
\hline
\end{tabular}

${ }^{1}$ Virus name: CIYVV: Clover yellow vein virus; ENMV: Endive necrotic mosaic virus; MeRV: Mediterranean ruda virus; WHMV: Wild hop mosaic virus

${ }^{2}$ Locations are designed with a three-letter code: ESC, El Escorial; MAR: Marjaliza; PAL: Palazuelos; PAR: EI Pardo; POL, Pollos; SCR, Santa Cruz de Retamar. 
Table A1.4. Continued.

\begin{tabular}{|c|c|c|c|c|c|c|}
\hline Isolate name & Acc № & Virus $^{1}$ & Sequenced & Location $^{2}$ & Host & Year \\
\hline WHMV-POL014_Hlu_PRI_2016 & Unpublished & WHMV & 350-nt Nlb & POL & Humulus Iupulus & 2016 \\
\hline WHMV-POL016_Hlu_VER_2013 & Unpublished & WHMV & 350-nt Nlb & POL & Humulus lupulus & 2013 \\
\hline WHMV-POL019_Hlu_PRI_2015 & Unpublished & WHMV & 350-nt Nlb/CP & POL & Humulus Iupulus & 2015 \\
\hline WHMV-POL020_Hlu_PRI_2015 & Unpublished & WHMV & 350-nt Nlb & POL & Humulus lupulus & 2015 \\
\hline WHMV-POL022_Eel_VER_2015 & Unpublished & WHMV & 350-nt Nlb & POL & Ecballium elaterium & 2015 \\
\hline WHMV-POL024 Hlu PRI 2016 & Unpublished & WHMV & 350-nt NIb & POL & Humulus lupulus & 2016 \\
\hline WHMV-POL042_Hlu_VER_2013 & Unpublished & WHMV & 350-nt NIb & POL & Humulus lupulus & 2013 \\
\hline WHMV-POL090_Eel_PRI_2014 & Unpublished & WHMV & 350-nt NIb & POL & Ecballium elaterium & 2014 \\
\hline WHMV-POL118_HIu_VER_2013 & Unpublished & WHMV & 350-nt NIb & POL & Humulus lupulus & 2013 \\
\hline WHMV-POL192_Hlu_VER_2013 & Unpublished & WHMV & 350-nt NIb & POL & Humulus Iupulus & 2013 \\
\hline WHMV-POLP93 Hlu PRI 2015 & Unpublished & WHMV & 350-nt NIb & POL & Humulus lupulus & 2015 \\
\hline WHMV-SCR018_Hlu_OTŌ2015 & Unpublished & WHMV & 350-nt Nlb & SCR & Humulus lupulus & 2015 \\
\hline WHMV-SCR077_Hlu_PRI_2014 & Unpublished & WHMV & 350-nt NIb/CP & SCR & Humulus Iupulus & 2014 \\
\hline WHMV-SCR077_Hlu_PRI_2016 & Unpublished & WHMV & 350-nt Nlb & SCR & Humulus Iupulus & 2016 \\
\hline WHMV-SCR101_HIu_PRI_2014 & Unpublished & WHMV & 350-nt NIb & SCR & Humulus Iupulus & 2014 \\
\hline WHMV-SCR152_HIU_PRI_2015 & Unpublished & WHMV & 350-nt Nlb & SCR & Humulus lupulus & 2015 \\
\hline WHMV-SCR98_Sat_VER_2013 & Unpublished & WHMV & 350-nt NIb & SCR & Salix atrocinerea & 2013 \\
\hline
\end{tabular}

1Virus name: CIYVV: Clover yellow vein virus; ENMV: Endive necrotic mosaic virus; MeRV: Mediterranean ruda virus; WHMV: Wild hop mosaic virus

${ }^{2}$ Locations are designed with a three-letter code: ESC, El Escorial; MAR: Marjaliza; PAL: Palazuelos; PAR: EI Pardo; POL, Pollos; SCR, Santa Cruz de Retamar. 
Table A1.5. Species of the genus Potyvirus compiled from GenBank used in the phylogenetic analyses.

\begin{tabular}{|c|c|}
\hline GenBank Acc. number & Virus name \\
\hline NC_010736 & Algerian watermelon mosaic virus \\
\hline NC_014905 & Apium virus $Y$ \\
\hline NC_018176 & Arracacha mottle virus \\
\hline NC_025821 & Asparagus virus 1 \\
\hline NC_009745 & Banana bract mosaic virus \\
\hline NC_009741 & Basella rugose mosaic virus \\
\hline NC_004047 & Bean common mosaic necrosis virus \\
\hline NC_003397 & Bean common mosaic virus \\
\hline NC_003492 & Bean yellow mosaic virus \\
\hline NC_005304 & Beet mosaic virus \\
\hline NC_023014 & Bidens mosaic virus \\
\hline NC_014325 & Bidens mottle virus \\
\hline NC_019415 & Blue squill virus $A$ \\
\hline NC_030847 & Brazilian weed virus $Y$ \\
\hline NC_014536 & Brugmansia suaveolens mottle virus \\
\hline NC_021196 & Calla lily latent virus \\
\hline NC_030794 & Callistephus mottle virus \\
\hline NC_013261 & Canna Yellow Streak Virus \\
\hline NC_025254 & Carrot thin leaf virus \\
\hline NC_027210 & Catharanthus mosaic virus \\
\hline NC_015393 & Celery mosaic virus \\
\hline NC_016044 & Chilli ringspot virus \\
\hline NC_005778 & Chilli veinal mottle virus \\
\hline NC_003536 & Clover yellow vein virus \\
\hline NC_003742 & Cocksfoot streak virus \\
\hline NC_020072 & Colombian datura virus \\
\hline NC_004013 & Cowpea aphid-borne mosaic virus \\
\hline NC_008028 & Daphne mosaic virus \\
\hline NC_003537 & Dasheen mosaic virus \\
\hline NC_021197 & Donkey orchid virus $A$ \\
\hline NC_007728 & East Asian Passiflora virus \\
\hline NC_034273 & Endive necrotic mosaic virus \\
\hline NC_031339 & Euphorbia ringspot virus \\
\hline NC_014064 & Freesia mosaic virus \\
\hline NC_010954 & Fritillary virus $Y$ \\
\hline NC_021786 & Habenaria mosaic virus \\
\hline NC_015394 & Hardenbergia mosaic virus \\
\hline NC_017967 & Hippeastrum mosaic virus \\
\hline NC_030236 & Impatiens flower break potyvirus \\
\hline NC_018833 & Iranian johnsongrass mosaic virus \\
\hline NC_029076 & Iris severe mosaic virus \\
\hline NC_000947 & Japanese yam mosaic virus \\
\hline NC_029051 & Jasmine ringspot virus \\
\hline NC_003606 & Johnsongrass mosaic virus \\
\hline NC_007913 & Konjac mosaic virus \\
\hline NC_004011 & Leek yellow stripe virus \\
\hline NC_003605 & Lettuce mosaic virus \\
\hline NC_005288 & Lily mottle virus \\
\hline NC_014898 & Lupine mosaic virus \\
\hline NC_003377 & Maize dwarf mosaic virus \\
\hline NC_009995 & Moroccan watermelon mosaic virus \\
\hline NC_008824 & Narcissus degeneration virus \\
\hline NC_023628 & Narcissus late season yellows virus \\
\hline NC_011541 & Narcissus yellow stripe virus \\
\hline NC_005029 & Onion yellow dwarf virus \\
\hline NC_019409 & Ornithogalum mosaic virus \\
\hline NC_014252 & Panax virus $Y$ \\
\hline NC_005028 & Papaya leaf-distortion mosaic potyvirus \\
\hline NC_001785 & Papaya ringspot virus \\
\hline NC_014790 & Passion fruit woodiness virus \\
\hline
\end{tabular}


Table A1.5. Continued.

\begin{tabular}{|c|c|}
\hline GenBank Acc. number & Virus name \\
\hline NC_001671 & Pea seed-borne mosaic virus \\
\hline NC_002600 & Peanut mottle virus \\
\hline NC 007147 & Pennisetum mosaic virus \\
\hline NC_001517 & Pepper mottle virus \\
\hline NC_008393 & Pepper severe mosaic virus \\
\hline NC_011918 & Pepper veinal mottle virus \\
\hline NC_014327 & Pepper yellow mosaic virus \\
\hline NC_004573 & Peru tomato mosaic virus \\
\hline NC_001445 & Plum pox virus \\
\hline NC_ 018872 & Pokeweed mosaic virus \\
\hline NC_004039 & Potato virus $A$ \\
\hline NC_004010 & Potato virus $V$ \\
\hline NC_001616 & Potato virus $Y$ \\
\hline NC_003399 & Scallion mosaic virus \\
\hline NC_007433 & Shallot yellow stripe virus \\
\hline NC_004035 & Sorghum mosaic virus \\
\hline NC_002634 & Soybean mosaic virus \\
\hline NC_003398 & Sugarcane mosaic virus \\
\hline NC_014038 & Sunflower chlorotic mottle virus \\
\hline NC_034208 & Sunflower ring blotch virus \\
\hline NC_001841 & Sweet potato feathery mottle virus \\
\hline NC_020896 & Sweet potato latent virus \\
\hline NC_017970 & Sweet potato virus 2 \\
\hline NC_014742 & Sweet potato virus $C$ \\
\hline NC_018093 & Sweet potato virus $G$ \\
\hline NC_026615 & Tamarillo leaf malformation virus \\
\hline NC 009742 & Telosma mosaic virus \\
\hline NC_007180 & Thunberg fritillary virus \\
\hline NC_001555 & Tobacco etch virus \\
\hline NC_009994 & Tobacco vein banding mosaic virus \\
\hline NC_001768 & Tobacco vein mottling virus \\
\hline NC_017824 & Tomato necrotic stunt virus \\
\hline NC_002509 & Turnip mosaic virus \\
\hline NC_017977 & Vallota speciosa virus \\
\hline NC_025250 & Vanilla distortion mosaic virus \\
\hline NC_010735 & Verbena virus $Y$ \\
\hline NC_006262 & Watermelon mosaic virus \\
\hline NC_004426 & Wild potato mosaic virus \\
\hline NC_009744 & Wild tomato mosaic virus \\
\hline NC_007216 & Wisteria vein mosaic virus \\
\hline NC_016441 & Yam bean mosaic virus \\
\hline NC_019412 & Yam mild mosaic virus \\
\hline NC_004752 & Yam mosaic virus \\
\hline NC_011560 & Zantedeschia mild mosaic virus \\
\hline NC_023175 & Zucchini tigre mosaic virus \\
\hline NC_003224 & Zucchini yellow mosaic virus \\
\hline
\end{tabular}




\section{APPENDIX 2}

Characterization of evergreen oak forest and riparian forests in the Iberian Peninsula 

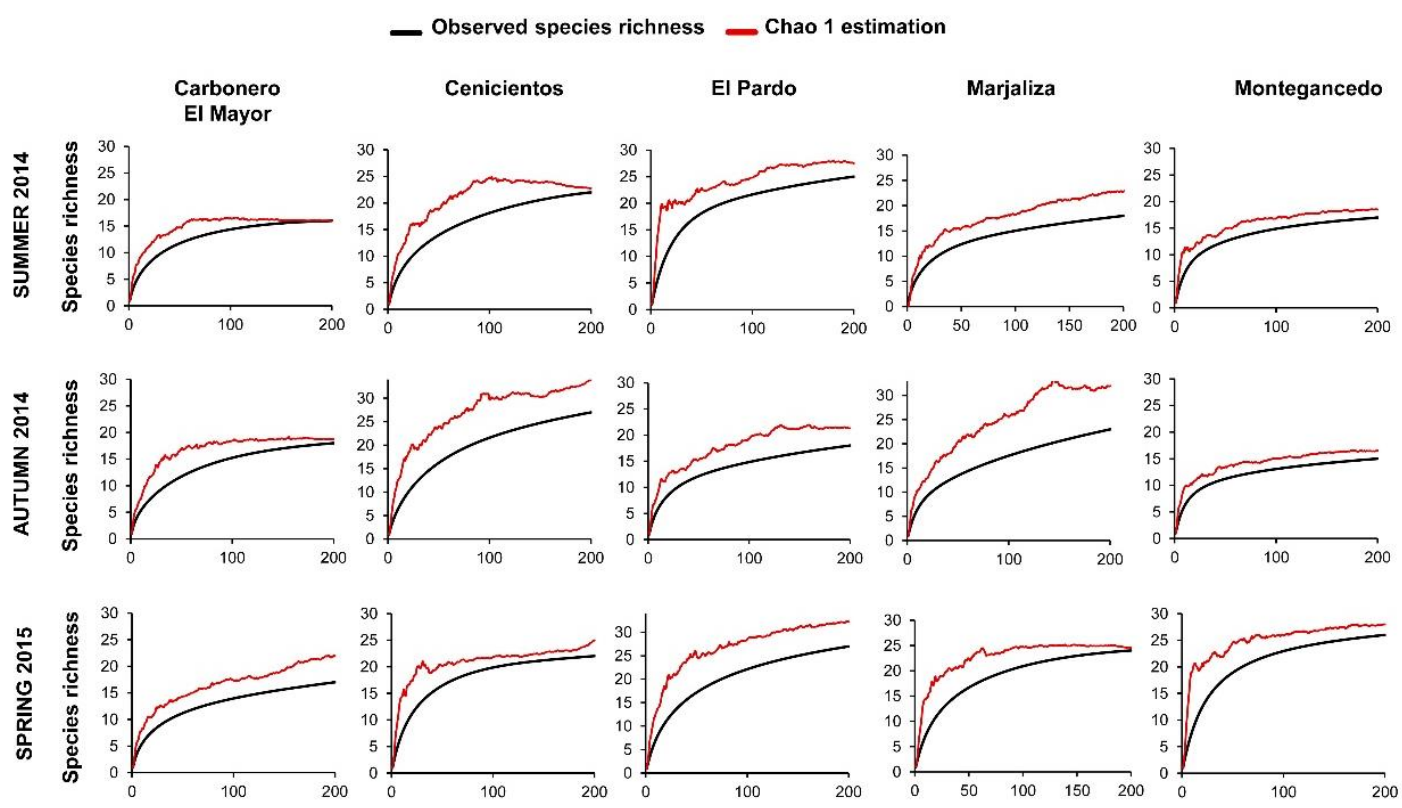

Number of individuals

Figure A2.1. Observed (Sobs, black) and expected (Sest, Chao 1 estimator, red) accumulation curves of plant species richness in the studied locations of evergreen oak forest for the monitored seasons during the 2014-2015 field samplings. The x-axis is the number of individuals sampled at each location (in this thesis, $\mathrm{N}=200$ ) and the $\mathrm{y}$-axis is the number of plant species collected according to sample size. Plotted values are means of 100 randomizations.

Table A2.1. Plant biodiversity in the studied evergreen oak forest locations during 2014-2015 field samplings. Observed and expected plant species richness, and Shannon's diversity and equitability indexes are shown.

\begin{tabular}{cccccc}
\hline Location & Season & $\mathbf{S}_{\text {obs }}(\mathbf{9 5 \%} \mathbf{C l})^{*}$ & $\mathbf{S}_{\text {est }}(\mathbf{9 5 \%} \mathbf{C l})^{*}$ & $\mathbf{H}^{\prime}$ & $\mathbf{E}_{\mathbf{H}}$ \\
\hline Carbonero & Summer 2014 & $16(15.21-16.79)$ & $16.00(16.71-17.23)$ & $2.12 \pm 0.00$ & 0.76 \\
el Mayor & Autumn 2014 & $18(15.70-20.30)$ & $18.75(18.07-26.42)$ & $2.05 \pm 0.00$ & 0.71 \\
& Spring 2015 & $17(11.75-22.25)$ & $21.98(17.77-48.99)$ & $2.07 \pm 0.00$ & 0.72 \\
Cenicientos & Summer 2014 & $22(19.96-24.04)$ & $22.75(22.08-29.20)$ & $2.37 \pm 0.00$ & 0.77 \\
& Autumn 2014 & $27(21.59-32.41)$ & $33.97(28.44-60.60)$ & $2.53 \pm 0.00$ & 0.77 \\
El Pardo & Spring 2015 & $22(18.82-25.18)$ & $24.99(22.35-47.61)$ & $2.72 \pm 0.00$ & 0.88 \\
& Summer 2014 & $25(21.30-28.70)$ & $27.49(25.37-41.91)$ & $2.89 \pm 0.00$ & 0.90 \\
& Autumn 2014 & $18(13.79-22.21)$ & $21.32(18.50-39.97)$ & $2.25 \pm 0.00$ & 0.78 \\
Marjaliza & Spring 2015 & $27(22.10-31.90)$ & $32.22(27.99-54.48)$ & $2.74 \pm 0.00$ & 0.83 \\
& Summer 2014 & $18(12.68-23.32)$ & $22.98(18.77-49.99)$ & $2.26 \pm 0.00$ & 0.78 \\
& Autumn 2014 & $23(17.29-28.71)$ & $31.96(24.99-63.27)$ & $2.42 \pm 0.00$ & 0.77 \\
& Spring 2015 & $24(21.97-26.03)$ & $24.60(24.05-31.08)$ & $2.71 \pm 0.00$ & 0.85 \\
Montegancedo & Summer 2014 & $17(13.31-20.69)$ & $18.49(17.15-32.01)$ & $2.41 \pm 0.00$ & 0.85 \\
& Autumn 2014 & $15(11.36-18.64)$ & $16.49(15.15-30.01)$ & $2.27 \pm 0.00$ & 0.84 \\
& Spring 2015 & $26(22.36-29.64)$ & $27.99(26.25-41.94)$ & $2.95 \pm 0.00$ & 0.90 \\
\hline
\end{tabular}

Sobs: observed plant species richness. Sest: expected plant species richness based on the Chao 1 estimator. H': Shannon's diversity index. $\mathrm{E}_{\mathrm{H}}$ : Shannon's equitability index.

*values are mean and 95\% confidence intervals based in 100 randomizations from pooled location-specific individuals $(\mathrm{N}=200)$. 


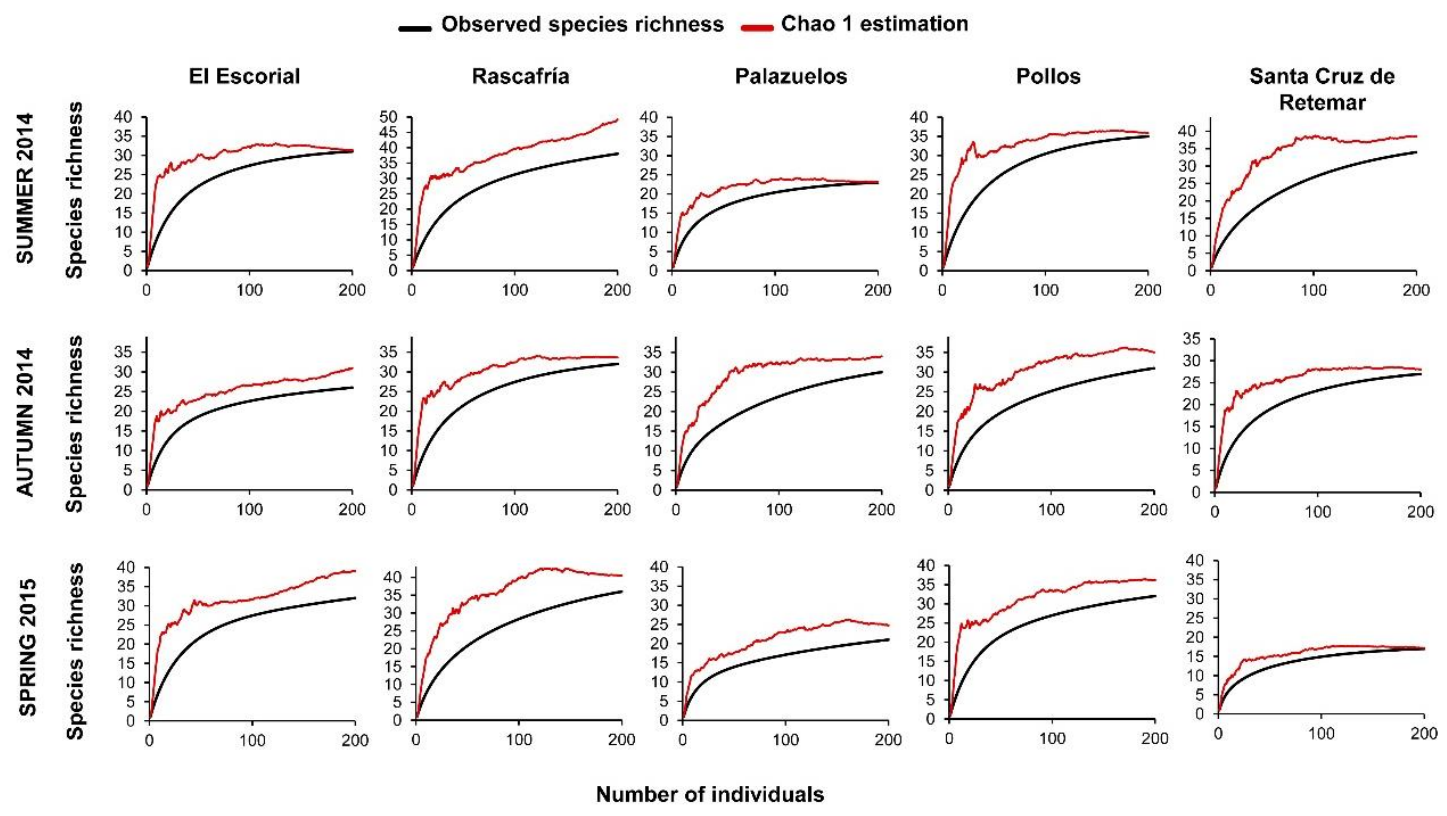

Figure A2.2. Observed ( $\mathrm{S}_{\text {obs}}$, black) and expected ( $\mathrm{S}_{\text {est, }}$ Chao 1 estimator, red) accumulation curves of plant species richness in the studied locations of riparian forest for the monitored seasons during the 2014-2015 field samplings. The $x$-axis is the number of individuals sampled at each location (in this thesis, $\mathrm{N}=200$ ) and the $\mathrm{y}$-axis is the number of plant species collected according to sample size. Plotted values are means of 100 randomizations.

Table A2.2. Plant biodiversity in the studied riparian forest locations during 2014-2015 field samplings. Observed and expected plant species richness, and Shannon's diversity and equitability indexes are shown.

\begin{tabular}{cccccc}
\hline Location & Season & $\mathbf{S}_{\text {obs }}(\mathbf{9 5 \%} \mathbf{C l})^{\star}$ & $\mathbf{S}_{\text {est }}(\mathbf{9 5 \%} \mathbf{C l})^{\star}$ & $\mathbf{H}^{\prime}$ & $\mathbf{E}_{\mathbf{H}}$ \\
\hline El Escorial & Summer 2014 & $31(29.33-32.67)$ & $31.43(31.03-36.55)$ & $3.16 \pm 0.00$ & 0.92 \\
& Autumn 2014 & $26(20.31-31.69)$ & $30.98(26.77-57.99)$ & $2.93 \pm 0.00$ & 0.90 \\
& Spring 2015 & $32(26.18-37.82)$ & $38.97(33.33-68.37)$ & $3.13 \pm 0.00$ & 0.90 \\
Rascafría & Summer 2014 & $38(31.34-44.66)$ & $49.19(40.64-85.50)$ & $3.30 \pm 0.00$ & 0.91 \\
& Autumn 2014 & $32(29.02-34.98)$ & $33.66(32.23-43.93)$ & $3.12 \pm 0.00$ & 0.90 \\
& Spring 2015 & $36(31.71-40.29)$ & $40.48(37.03-55.53)$ & $3.01 \pm 0.00$ & 0.84 \\
Palazuelos & Summer 2014 & $23(21.77-24.23)$ & $23.17(23.01-26.58)$ & $2.76 \pm 0.00$ & 0.88 \\
& Autumn 2014 & $30(25.84-34.16)$ & $33.98(30.80-49.72)$ & $2.83 \pm 0.00$ & 0.83 \\
& Spring 2015 & $21(16.77-25.23)$ & $24.73(21.63-42.92)$ & $2.50 \pm 0.00$ & 0.82 \\
Sallos & Summer 2014 & $35(32.78-37.22)$ & $35.85(35.09-42.94)$ & $3.26 \pm 0.00$ & 0.92 \\
& Autumn 2014 & $31(26.83-35.17)$ & $34.98(31.80-50.72)$ & $2.98 \pm 0.00$ & 0.87 \\
de Retamar & Spring 2015 & $32(27.57-36.43)$ & $36.18(32.79-54.24)$ & $3.14 \pm 0.00$ & 0.90 \\
& Summer 2014 & $34(29.65-38.35)$ & $38.48(34.97-54.61)$ & $2.80 \pm 0.00$ & 0.79 \\
& Autumn 2014 & $27(24.60-29.40)$ & $28.00(27.11-35.94)$ & $2.86 \pm 0.00$ & 0.87 \\
& Spring 2015 & $17(15.43-18.57)$ & $17.25(17.01-21.78)$ & $2.24 \pm 0.00$ & 0.79 \\
\hline
\end{tabular}

Sobs: observed plant species richness. Sest: expected plant species richness based on the Chao 1 estimator. H': Shannon's diversity index. $E_{H}$ : Shannon's equitability index.

*values are mean and 95\% confidence intervals based in 100 randomizations from pooled location-specific individuals $(\mathrm{N}=200)$. 
Table A2.3. Identified plant families and species in evergreen oak forest populations during 2013-2015 field samplings.

\begin{tabular}{|c|c|c|}
\hline Plant Family & Plant species & Life Cycle \\
\hline Anacardiaceae & Pistacia terebinthus & perennial \\
\hline Apiaceae & $\begin{array}{l}\text { Anthriscus caucalis } \\
\text { Daucus carota } \\
\text { Eryngium campestre } \\
\text { Pimpinella villosa } \\
\text { Scandix pecten-veneris } \\
\text { Thapsia villosa } \\
\text { Torilis leptophylla }\end{array}$ & $\begin{array}{l}\text { annual } \\
\text { perennial } \\
\text { perennial } \\
\text { perennial } \\
\text { annual } \\
\text { perennial } \\
\text { annual }\end{array}$ \\
\hline Araliaceae & Hedera helix & perennial \\
\hline Asparagaceae & Asparagus acutifolius & perennial \\
\hline Asteraceae & $\begin{array}{l}\text { Andryala arenaria } \\
\text { Andryala integrifolia } \\
\text { Andryala ragusina } \\
\text { Aster arvensis } \\
\text { Bellis perennis } \\
\text { Bombycilaena discolor } \\
\text { Carduus tenuiflorus } \\
\text { Centaurea melitensis } \\
\text { Centaurea paniculata } \\
\text { Conyza andryala } \\
\text { Crepis capillaris } \\
\text { Crepis taraxacifolia } \\
\text { Crupina vulgaris } \\
\text { Filago arvensis } \\
\text { Helichrysum stoechas } \\
\text { Hieracium sp. } \\
\text { Lactuca serriola } \\
\text { Lactuca virosa } \\
\text { Leontodon longirostris } \\
\text { Phagnalon saxatile } \\
\text { Picris echioides } \\
\text { Santolina rosmarinifolia } \\
\text { Scolymus hispanicus } \\
\text { Senecio jacobaea } \\
\text { Sonchus oleraceus } \\
\text { Taraxacum officinale } \\
\text { Tolpis umbellata } \\
\end{array}$ & $\begin{array}{l}\text { annual } \\
\text { perennial } \\
\text { perennial } \\
\text { annual } \\
\text { perennial } \\
\text { annual } \\
\text { perennial } \\
\text { annual } \\
\text { perennial } \\
\text { annual } \\
\text { annual } \\
\text { perennial } \\
\text { annual } \\
\text { annual } \\
\text { perennial } \\
\text { perennial } \\
\text { annual } \\
\text { annual } \\
\text { annual } \\
\text { perennial } \\
\text { perennial } \\
\text { perennial } \\
\text { perennial } \\
\text { perennial } \\
\text { annual } \\
\text { perennial } \\
\text { annual } \\
\end{array}$ \\
\hline Boraginaceae & $\begin{array}{l}\text { Anchusa azurea } \\
\text { Anchusa undulata } \\
\text { Buglossoides arvensis } \\
\text { Echium plantagineum } \\
\text { Echium sp. } \\
\text { Echium vulgare } \\
\text { Helioptropium europaeum } \\
\text { Heliotropium europaeum }\end{array}$ & $\begin{array}{l}\text { perennial } \\
\text { perennial } \\
\text { annual } \\
\text { annual } \\
\text { annual } \\
\text { annual } \\
\text { annual } \\
\text { annual }\end{array}$ \\
\hline Brassicaceae & $\begin{array}{l}\text { Brassica barrelieri } \\
\text { Diplotaxis virgata } \\
\text { Iberis ciliata } \\
\text { Malcolmia patula } \\
\text { Malcolmia triloba }\end{array}$ & $\begin{array}{l}\text { annual } \\
\text { annual } \\
\text { perennial } \\
\text { annual } \\
\text { annual }\end{array}$ \\
\hline Campanulaceae & $\begin{array}{l}\text { Campanula rapunculus } \\
\text { Jasione montana }\end{array}$ & $\begin{array}{l}\text { perennial } \\
\text { annual }\end{array}$ \\
\hline Caryophyllaceae & $\begin{array}{l}\text { Arenaria montana } \\
\text { Holosteum umbellatum } \\
\text { Petrorhagia nanteuillii }\end{array}$ & $\begin{array}{l}\text { perennial } \\
\text { annual } \\
\text { annual }\end{array}$ \\
\hline
\end{tabular}


Table A2.3. Continued.

\begin{tabular}{|c|c|c|}
\hline Plant Family & Plant species & Life Cycle \\
\hline Caryophyllaceae & $\begin{array}{l}\text { Silene colorata } \\
\text { Silene gallica } \\
\text { Stellaria media }\end{array}$ & $\begin{array}{l}\text { annual } \\
\text { annual } \\
\text { annual }\end{array}$ \\
\hline Cistaceae & $\begin{array}{l}\text { Cistus albidus } \\
\text { Cistus ladanifer } \\
\text { Cistus laurifolius } \\
\text { Cistus salviifolius } \\
\text { Halimium umbellatum } \\
\text { Halimium viscosum } \\
\text { Tuberaria guttata } \\
\end{array}$ & $\begin{array}{l}\text { perennial } \\
\text { perennial } \\
\text { perennial } \\
\text { perennial } \\
\text { perennial } \\
\text { perennial } \\
\text { annual } \\
\end{array}$ \\
\hline Convolvulaceae & Convolvulus arvensis & perennial \\
\hline Crassulaceae & $\begin{array}{l}\text { Sedum forsterianum } \\
\text { Sedum sediforme } \\
\text { Umbilicus rupestris }\end{array}$ & $\begin{array}{l}\text { perennial } \\
\text { perennial } \\
\text { perennial }\end{array}$ \\
\hline Cucurbitaceae & Ecballium elaterium & perennial \\
\hline Cupressaceae & Juniperus oxycedrus & perennial \\
\hline Dipsacaceae & Pterocephalidum diandra & annual \\
\hline Fabaceae & $\begin{array}{l}\text { Anthyllis cornicina } \\
\text { Anthyllis lotoides } \\
\text { Anthyllis vulneraria subsp. gandogeri } \\
\text { Astragalus hamosus } \\
\text { Astragalus pelecinus } \\
\text { Biserrula pelecinus } \\
\text { Coronilla scorpioides } \\
\text { Cytisus scoparius } \\
\text { Hymenocarpos cornicina } \\
\text { Hymenocarpos lotoides } \\
\text { Lathyrus angulatus } \\
\text { Lathyrus cicera } \\
\text { Lotus corniculatus } \\
\text { Lupinus angustifolius } \\
\text { Lupinus hispanica } \\
\text { Lupinus sp. } \\
\text { Ornithopus compressus } \\
\text { Retama sphaerocarpa } \\
\text { Trifolium angustifolium } \\
\text { Trifolium arvense } \\
\text { Trifolium campestre } \\
\text { Trifolium cherleri } \\
\text { Trifolium hirtum } \\
\text { Trifolium scabrum } \\
\text { Trifolium sp. } \\
\text { Trifolium stellatum } \\
\text { Trifolium striatum } \\
\text { Trigonella monspeliaca } \\
\text { Vicia disperma } \\
\text { Vicia lutea } \\
\text { Vicia villosa }\end{array}$ & $\begin{array}{l}\text { annual } \\
\text { annual } \\
\text { annual } \\
\text { annual } \\
\text { annual } \\
\text { annual } \\
\text { annual } \\
\text { perennial } \\
\text { annual } \\
\text { annual } \\
\text { annual } \\
\text { annual } \\
\text { perennial } \\
\text { annual } \\
\text { annual } \\
\text { annual } \\
\text { annual } \\
\text { perennial } \\
\text { annual } \\
\text { annual } \\
\text { annual } \\
\text { annual } \\
\text { annual } \\
\text { annual } \\
\text { annual } \\
\text { annual } \\
\text { annual } \\
\text { annual } \\
\text { annual } \\
\text { annual } \\
\text { annual }\end{array}$ \\
\hline Fagaceae & $\begin{array}{l}\text { Quercus coccifera } \\
\text { Quercus ilex }\end{array}$ & $\begin{array}{l}\text { perennial } \\
\text { perennial }\end{array}$ \\
\hline Fumariaceae & Fumaria officinalis & annual \\
\hline Geraniaceae & $\begin{array}{l}\text { Erodium cicutarium } \\
\text { Geranium dissectum } \\
\text { Geranium molle } \\
\text { Geranium robertianum } \\
\text { Geranium rotundifolium }\end{array}$ & $\begin{array}{l}\text { annual } \\
\text { annual } \\
\text { annual } \\
\text { perennial } \\
\text { perennial }\end{array}$ \\
\hline
\end{tabular}


Table A2.3. Continued.

\begin{tabular}{|c|c|c|}
\hline Plant Family & Plant species & Life Cycle \\
\hline Lamiaceae & $\begin{array}{l}\text { Lavandula stoechas subsp. pedunculata } \\
\text { Thymus mastichina }\end{array}$ & $\begin{array}{l}\text { perennial } \\
\text { perennial }\end{array}$ \\
\hline Linaceae & Linum narbonense & perennial \\
\hline Moraceae & Ficus carica & annual \\
\hline Oleaceae & Fraxinus angustifolia & perennial \\
\hline Pinaceae & Pinus pinea & perennial \\
\hline Plantaginaceae & $\begin{array}{l}\text { Plantago bellardii } \\
\text { Plantago coronopus } \\
\text { Plantago lagopus } \\
\text { Plantago lanceolata }\end{array}$ & $\begin{array}{l}\text { annual } \\
\text { perennial } \\
\text { annual } \\
\text { perennial }\end{array}$ \\
\hline Poaceae & $\begin{array}{l}\text { Anthoxanthum odoratum } \\
\text { Avena barbata } \\
\text { Avena sterilis } \\
\text { Brachypodium sylvaticum } \\
\text { Briza maxima } \\
\text { Bromus diandrus } \\
\text { Bromus madritensis } \\
\text { Bromus mollis } \\
\text { Bromus rubens } \\
\text { Bromus tectorum } \\
\text { Carex sp. } \\
\text { Corynephorus fasciculatus } \\
\text { Cynodon dactylon } \\
\text { Dactylis glomerata } \\
\text { Dactylis hispanica } \\
\text { Holcus lanatus } \\
\text { Melica magnolii } \\
\text { Piptatherum miliaceum } \\
\text { Poa pratensis } \\
\text { Taeniatherum caput-medusae } \\
\text { Vulpia myuros }\end{array}$ & $\begin{array}{l}\text { annual } \\
\text { annual } \\
\text { annual } \\
\text { perennial } \\
\text { annual } \\
\text { annual } \\
\text { perennial } \\
\text { annual } \\
\text { annual } \\
\text { perennial } \\
\text { perennial } \\
\text { annual } \\
\text { perennial } \\
\text { perennial } \\
\text { perennial } \\
\text { perennial } \\
\text { perennial } \\
\text { perennial } \\
\text { perennial } \\
\text { annual } \\
\text { annual }\end{array}$ \\
\hline Polygonaceae & $\begin{array}{l}\text { Rumex acetosa } \\
\text { Rumex angiocarpus } \\
\text { Rumex bucephalophorus } \\
\text { Rumex gallicus }\end{array}$ & $\begin{array}{l}\text { perennial } \\
\text { perennial } \\
\text { annual } \\
\text { annual }\end{array}$ \\
\hline $\begin{array}{l}\text { Ranunculaceae } \\
\text { Rosaceae }\end{array}$ & $\begin{array}{l}\text { Ranunculus bulbosus } \\
\text { Ranunculus ollisiponensis } \\
\text { Sanguisorba minor subsp. spachiana }\end{array}$ & $\begin{array}{l}\text { perennial } \\
\text { perennial } \\
\text { perennial }\end{array}$ \\
\hline Rubiaceae & $\begin{array}{l}\text { Galium aparine } \\
\text { Sherardia arvensis }\end{array}$ & $\begin{array}{l}\text { annual } \\
\text { annual }\end{array}$ \\
\hline Rutaceae & Ruta montana & perennial \\
\hline Scrophulariaceae & $\begin{array}{l}\text { Bellardia trixago } \\
\text { Linaria spartea } \\
\text { Parentucellia latifolia }\end{array}$ & $\begin{array}{l}\text { annual } \\
\text { annual } \\
\text { annual }\end{array}$ \\
\hline Thymelaceae & Daphne gnidium & perennial \\
\hline Violaceae & Viola kitaibeliana & annual \\
\hline
\end{tabular}


Table A2.4. Identified plant families and species in Riparian forest populations during 20132015 field samplings.

\begin{tabular}{|c|c|c|}
\hline Plant Family & Plant species & Life Cycle \\
\hline \multirow[t]{10}{*}{ Apiaceae } & Anthriscus caucalis & annual \\
\hline & Conopodium subcarneum & perennial \\
\hline & Daucus carota & perennial \\
\hline & Eryngium campestre & perennial \\
\hline & Myrrhoides nodosa & annual \\
\hline & Peucedanum sp. & perennial \\
\hline & Physospermum cornubiense & perennial \\
\hline & Thapsia villosa & perennial \\
\hline & Torilis arvensis & annual \\
\hline & Torilis japonica & annual \\
\hline \multirow[t]{2}{*}{ Apocynaceae } & Vinca minor & perennial \\
\hline & Vincetoxicum nigrum & perennial \\
\hline Araceae & Arum italicum & perennial \\
\hline Araliaceae & Hedera helix & perennial \\
\hline \multirow[t]{2}{*}{ Aristolochiaceae } & Aristolochia longa & perennial \\
\hline & Aristolochia paucinervis & perennial \\
\hline Asparagaceae & Asparagus acutifolius & perennial \\
\hline \multirow[t]{29}{*}{ Asteraceae } & Achillea filipendulina & perennial \\
\hline & Andryala arenaria & annual \\
\hline & Andryala integrifolia & perennial \\
\hline & Andryala ragusina & perennial \\
\hline & Artemisia absinthium & perennial \\
\hline & Aster arvensis & annual \\
\hline & Bellis perennis & perennial \\
\hline & Carduus tenuiflorus & perennial \\
\hline & Centaurea calcitrapa & perennial \\
\hline & Centaurea melitensis & annual \\
\hline & Chondrilla juncea & perennial \\
\hline & Cichorium intybus & perennial \\
\hline & Conyza bonariensis & annual \\
\hline & Conyza canadensis & annual \\
\hline & Filago arvensis & annual \\
\hline & Helichrysum stoechas & perennial \\
\hline & Hieracium murorum & perennial \\
\hline & Hieracium pilosella & perennial \\
\hline & Hieracium sabaudum & perennial \\
\hline & Jasonia tuberosa & perennial \\
\hline & Lactuca serriola & annual \\
\hline & Lactuca virosa & annual \\
\hline & Lapsana communis & annual \\
\hline & Leontodon longirostris & annual \\
\hline & Senecio jacobaea & perennial \\
\hline & Tanacetum corymbosum & perennial \\
\hline & Tanacetum parthenium & perennial \\
\hline & Tanacetum vulgare & perennial \\
\hline & Taraxacum officinale & perennial \\
\hline \multirow[t]{8}{*}{ Boraginaceae } & Anchusa azurea & perennial \\
\hline & Anchusa undulata & perennial \\
\hline & Buglossoides arvensis & annual \\
\hline & Cynoglossum officinale & perennial \\
\hline & Echium vulgare & annual \\
\hline & Myosotis arvensis & annual \\
\hline & Pentaglottis sempervirens & perennial \\
\hline & Symphytum tuberosum & perennial \\
\hline Brassicaceae & Alliaria petiolata & perennial \\
\hline
\end{tabular}


Table A2.4. Continued.

\begin{tabular}{|c|c|c|}
\hline Plant Family & Plant species & Life Cycle \\
\hline \multirow[t]{9}{*}{ Brassicaceae } & Brassica barrelieri & annual \\
\hline & Capsella bursa-pastoris & annual \\
\hline & Cardamine hirsuta & annual \\
\hline & Eruca vesicaria & annual \\
\hline & Malcolmia littorea & perennial \\
\hline & Malcolmia patula & annual \\
\hline & Malcolmia triloba & annual \\
\hline & Sisymbrium austriacum & perennial \\
\hline & Sisymbrium irio & annual \\
\hline Cannabaceae & Humulus Iupulus & perennial \\
\hline \multirow[t]{3}{*}{ Caprifoliaceae } & Lonicera hispanica & perennial \\
\hline & Lonicera periclymenum & perennial \\
\hline & Lonicera xylosteum & perennial \\
\hline \multirow[t]{12}{*}{ Caryophyllaceae } & Arenaria montana & perennial \\
\hline & Cerastium arvense & perennial \\
\hline & Dianthus armeria & annual \\
\hline & Holosteum umbellatum & annual \\
\hline & Lychnis flos-cuculi & perennial \\
\hline & Petrorhagia nanteuillii & annual \\
\hline & Saponaria officinalis & perennial \\
\hline & Silene alba & perennial \\
\hline & Silene mellifera & perennial \\
\hline & Silene vulgaris & perennial \\
\hline & Stellaria graminea & perennial \\
\hline & Stellaria media & annual \\
\hline \multirow[t]{2}{*}{ Chenopodiaceae } & Chenopodium album & annual \\
\hline & Chenopodium opulifolium & annual \\
\hline \multirow[t]{2}{*}{ Convolvulaceae } & Calystegia sepium & perennial \\
\hline & Convolvulus arvensis & perennial \\
\hline \multirow[t]{2}{*}{ Cucurbitaceae } & Bryonia dioica & perennial \\
\hline & Ecballium elaterium & perennial \\
\hline \multirow[t]{4}{*}{ Cyperaceae } & Carex broteriana & perennial \\
\hline & Carex elata subsp. reuteriana & perennial \\
\hline & Cyperus longus & perennial \\
\hline & Scirpus holoschoenus & perennial \\
\hline Dioscoreaceae & Tamus communis & perennial \\
\hline Fabaceae & Adenocarpus complicatus & perennial \\
\hline \multirow[t]{21}{*}{ Fabaceae } & Astragalus glycyphyllos & perennial \\
\hline & Astragalus hamosus & annual \\
\hline & Cytisus scoparius & perennial \\
\hline & Genista florida & perennial \\
\hline & Lathyrus angulatus & annual \\
\hline & Lathyrus aphaca & annual \\
\hline & Lathyrus cicera & annual \\
\hline & Lathyrus pratensis & perennial \\
\hline & Lotus corniculatus & perennial \\
\hline & Lupinus angustifolius & annual \\
\hline & Medicago littoralis & annual \\
\hline & Medicago minima & annual \\
\hline & Medicago sativa & perennial \\
\hline & Melilotus indicus & annual \\
\hline & Ornithopus compressus & annual \\
\hline & Retama sphaerocarpa & perennial \\
\hline & Trifolium arvense & annual \\
\hline & Trifolium campestre & annual \\
\hline & Trifolium glomeratum & annual \\
\hline & Trifolium pratense & perennial \\
\hline & Trifolium repens & perennial \\
\hline
\end{tabular}


Table A2.4. Continued.

\begin{tabular}{|c|c|c|}
\hline Plant Family & Plant species & Life Cycle \\
\hline \multirow[t]{8}{*}{ Fabaceae } & Trifolium scabrum & annual \\
\hline & Vicia disperma & annual \\
\hline & Vicia hybrida & annual \\
\hline & Vicia lathyroides & annual \\
\hline & Vicia orobus & perennial \\
\hline & Vicia sativa & annual \\
\hline & Vicia tenuifolia & perennial \\
\hline & Vicia villosa & annual \\
\hline Fagaceae & Quercus pyrenaica & perennial \\
\hline \multirow[t]{2}{*}{ Fumariaceae } & Fumaria officinalis & annual \\
\hline & Fumaria parviflora & annual \\
\hline \multirow[t]{5}{*}{ Geraniaceae } & Erodium cicutarium & annual \\
\hline & Geranium dissectum & annual \\
\hline & Geranium molle & annual \\
\hline & Geranium robertianum & perennial \\
\hline & Geranium rotundifolium & perennial \\
\hline Guttiferae & Hypericum perforatum & perennial \\
\hline Juglandaceae & Juglans regia & perennial \\
\hline \multirow[t]{8}{*}{ Lamiaceae } & Clinopodium vulgare & perennial \\
\hline & Lamium album & perennial \\
\hline & Lamium purpureum & annual \\
\hline & Lycopus europaeus & perennial \\
\hline & Marrubium vulgare & perennial \\
\hline & Mentha longifolia & perennial \\
\hline & Mentha pulegium & perennial \\
\hline & Mentha rotundifolia & perennial \\
\hline \multirow[t]{8}{*}{ Lamiaceae } & Nepeta caerulea & perennial \\
\hline & Nepeta cataria & perennial \\
\hline & Nepeta nepetella & perennial \\
\hline & Origanum virens & perennial \\
\hline & Salvia verbenaca & perennial \\
\hline & Satureja montana & perennial \\
\hline & Teucrium fruticans & perennial \\
\hline & Teucrium scorodonia & perennial \\
\hline Liliaceae & Asphodelus albus & perennial \\
\hline Lythraceae & Lythrum salicaria & perennial \\
\hline \multirow[t]{2}{*}{ Malvaceae } & Malva sylvestris & perennial \\
\hline & Malva tournefortiana & perennial \\
\hline Myrsinaceae & Lysimachia vulgaris & perennial \\
\hline \multirow[t]{2}{*}{ Oleaceae } & Fraxinus angustifolia & perennial \\
\hline & Ligustrum vulgare & perennial \\
\hline Onagraceae & Epilobium lanceolatum & perennial \\
\hline Oxalidaceae & Oxalis acetosella & perennial \\
\hline Papaveraceae & Papaver rhoeas & annual \\
\hline Pinaceae & Pinus pinea & perennial \\
\hline \multirow[t]{2}{*}{ Plantaginaceae } & Plantago lanceolata & perennial \\
\hline & Plantago media & perennial \\
\hline \multirow[t]{11}{*}{ Poaceae } & Anthoxanthum odoratum & annual \\
\hline & Arrhenatherum elatius & perennial \\
\hline & Avena barbata & annual \\
\hline & Brachypodium dystachion & annual \\
\hline & Brachypodium sylvaticum & perennial \\
\hline & Bromus diandrus & annual \\
\hline & Bromus madritensis & perennial \\
\hline & Catapodium rigidum & annual \\
\hline & Cynosurus cristatus & perennial \\
\hline & Cynosurus echinatus & annual \\
\hline & Dactylis glomerata & perennial \\
\hline
\end{tabular}


Table A2.4. Continued.

\begin{tabular}{|c|c|c|}
\hline Plant Family & Plant species & Life Cycle \\
\hline \multirow{13}{*}{ Poaceae } & Dactylis hispanica & perennial \\
\hline & Elymus caninus & perennial \\
\hline & Festuca arundinaceae & perennial \\
\hline & Festuca durandoi & perennial \\
\hline & Hordeum murinum & annual \\
\hline & Lolium rigidum & annual \\
\hline & Paspalum dilatatum & perennial \\
\hline & Phleum pratense & perennial \\
\hline & Phragmites australis & perennial \\
\hline & Piptatherum miliaceum & perennial \\
\hline & Poa pratensis & perennial \\
\hline & Stipa gigantea & perennial \\
\hline & Taeniatherum caput-medusae & annual \\
\hline \multirow{2}{*}{ Polygonaceae } & Rumex acetosa & perennial \\
\hline & Rumex bucephalophorus & annual \\
\hline \multirow[t]{4}{*}{ Polygonaceae } & Rumex conglomeratus & perennial \\
\hline & Rumex crispus & perennial \\
\hline & Rumex induratus & perennial \\
\hline & Rumex pulcher & perennial \\
\hline \multirow[t]{7}{*}{ Ranunculaceae } & Clematis vitalba & perennial \\
\hline & Nigella gallica & annual \\
\hline & Nigella nigellastrum & perennial \\
\hline & Ranunculus bulbosus & perennial \\
\hline & Ranunculus montserratii & perennial \\
\hline & Ranunculus ollisiponensis & perennial \\
\hline & Thalictrum flavum & perennial \\
\hline \multirow[t]{9}{*}{ Rosaceae } & Crataegus monogyna & perennial \\
\hline & Geum silvaticum & perennial \\
\hline & Malus sp. & perennial \\
\hline & Potentilla anserina & perennial \\
\hline & Potentilla argentea & perennial \\
\hline & Prunus spinosa & perennial \\
\hline & Rosa obtusifolia & perennial \\
\hline & Rubus sp. & perennial \\
\hline & Rubus ulmifolius & perennial \\
\hline \multirow[t]{9}{*}{ Rubiaceae } & Cruciata glabra & perennial \\
\hline & Cruciata laevipes & perennial \\
\hline & Galium aparine & annual \\
\hline & Galium aparine subsp. spurium & annual \\
\hline & Galium odoratum & perennial \\
\hline & Galium parisiense & annual \\
\hline & Galium rotundifolium & perennial \\
\hline & Galium scabrum & perennial \\
\hline & Galium verum & annual \\
\hline \multirow[t]{5}{*}{ Salicaceae } & Populus nigra & perennial \\
\hline & Populus sp. & perennial \\
\hline & Salix atrocinerea & perennial \\
\hline & Salix eleagnos & perennial \\
\hline & Salix triandra & perennial \\
\hline Saxifragaceae & Saxifraga granulata & perennial \\
\hline \multirow[t]{3}{*}{ Scrophulariaceae } & Verbascum sinuatum & perennial \\
\hline & Verbascum sp. & perennial \\
\hline & Veronica officinalis & perennial \\
\hline Simaroubaceae & Ailanthus altissima & annual \\
\hline Solanaceae & Solanum dulcamara & perennial \\
\hline Ulmaceae & Ulmus pumila & perennial \\
\hline
\end{tabular}


APPENDIX 2

Table A2.4. Continued.

Plant Family

Urticaceae

\begin{tabular}{l}
\hline Valerianaceae \\
\hline Violaceae
\end{tabular}
Plant species

Urtica dioica

Urtica urens

Valerianella coronata

Viola kitaibeliana

Viola odorata

Viola riviniana
Life Cycle

perennial

annual

annual

annual

perennial

perennial 


\section{APPENDIX 3}

Analysis of genus-wide potyvirus prevalence in wild ecosystems in the Iberian Peninsula 
Table A3.1. Logistic models predicting potyvirus prevalence closely competing with the best-ranked one $\left(\Delta_{i}<2\right)$, and/or the best model with considerable less support $\left(\Delta_{i}>2\right)$.

\begin{tabular}{|c|c|c|c|c|c|c|c|c|}
\hline \multirow[t]{2}{*}{ Model structure } & \multicolumn{2}{|c|}{$\begin{array}{l}\text { Model } \\
\text { fitting }\end{array}$} & \multicolumn{3}{|c|}{$\begin{array}{l}\text { Model performance } \\
\text { on } 5 \times 2 \mathrm{CV} \text { training set }^{3}\end{array}$} & \multicolumn{3}{|c|}{$\begin{array}{l}\text { Model performance } \\
\text { on validation set }\end{array}$} \\
\hline & $\mathrm{AIC}^{1}$ & $\Delta_{\mathrm{i}}^{2}$ & Acc & AUROC & TPR & $\overline{\text { Acc }}$ & AUROC & TPR \\
\hline \multicolumn{9}{|l|}{ Riparian forests } \\
\hline PSP (98)+ PRA (1)+SEA (1) & 1407.29 & 0.00 & 92 & 97 & 54 & 83 & 72 & 15 \\
\hline PSP (99) + PRA (1) & 1428.66 & 21.37 & ND & ND & ND & ND & ND & ND \\
\hline \multicolumn{9}{|l|}{ Evergreen oak forests } \\
\hline $\begin{array}{l}\mathrm{PSP}(96)+\mathrm{RAF}(2)+\mathrm{RH} \text { med }(1)+\mathrm{V}(1) \\
\operatorname{PSP}(98)+\mathrm{RAF}(1)+\mathrm{RH} H_{\text {med }}(1)\end{array}$ & $\begin{array}{l}906.96 \\
916.00\end{array}$ & $\begin{array}{l}0.00 \\
9.04\end{array}$ & $\begin{array}{r}95 \\
\text { ND }\end{array}$ & $\begin{array}{r}98 \\
\mathrm{ND}\end{array}$ & $\begin{array}{l}26 \\
\mathrm{ND}\end{array}$ & $\begin{array}{l}91 \\
\mathrm{ND}\end{array}$ & $\begin{array}{l}70 \\
\text { ND }\end{array}$ & $\begin{array}{l}20 \\
\text { ND }\end{array}$ \\
\hline
\end{tabular}

${ }^{*}$ The relative importance (\%) of each predictor variable is shown in parenthesis.

${ }^{1}$ Akaike's Information Criterion.

${ }^{2} \Delta i$, is the difference between the AIC of a given model and that of the best-ranked model, and quantifies how models compete (best-ranked model: $\Delta i=0$; substantial empirical support: $\Delta i=1-2$; considerable less support: $\Delta i=2-7 ;$ and no support; $\Delta i>10$ ) (Burnham and Anderson, 2002).

${ }^{3}$ Model performance measures: rate of correct predictions of the model (Acc: Accuracy), Area under the ROC curve (AUROC), True Positive rate (TPR). Values are shown as percentages. Measures calculated for models with $\triangle i<2$. ND: Not determined.

PSP: Plant species; PRA: Plant relative abundance; SEA: Season; RAF: Rainfalls; RHmed: Average relative humidity; V: Plant biomass.

Table A3.2. Logistic models predicting potyvirus prevalence excluding plant identity closely competing with the best-ranked one $\left(\Delta_{i}<2\right)$, and/or the best model with considerable less support $\left(\Delta_{i}>2\right)$.

\begin{tabular}{|c|c|c|c|c|c|c|c|c|}
\hline \multirow[t]{2}{*}{ Model structure* } & \multicolumn{2}{|c|}{$\begin{array}{l}\text { Model } \\
\text { fitting }\end{array}$} & \multicolumn{3}{|c|}{$\begin{array}{l}\text { Model performance } \\
\text { on } 5 \times 2 \mathrm{CV} \text { training set }\end{array}$} & \multicolumn{3}{|c|}{$\begin{array}{l}\text { Model performance } \\
\text { on validation set }^{3}\end{array}$} \\
\hline & AIC $^{1}$ & $\Delta_{\mathrm{i}}^{2}$ & Acc & AUROC & TPR & Acc & AUROC & TPR \\
\hline \multicolumn{9}{|l|}{ Riparian forests } \\
\hline PRA (46)+PLC (25)+HPS (21)+RAF (8) & 914.75 & 0.00 & 55 & 72 & 5 & 58 & 72 & 3 \\
\hline PRA (48)+PLC (22)+HPS (30) & 915.75 & 1.00 & 47 & 71 & 5 & 54 & 66 & 0 \\
\hline PRA (61)+PLC (39) & 932.52 & 17.77 & ND & ND & ND & ND & ND & ND \\
\hline \multicolumn{9}{|l|}{ Evergreen oak forests } \\
\hline PRA (80)+PLC (20) & 516.05 & 0.00 & 67 & 71 & 0 & 62 & 60 & 0 \\
\hline PRA (100) & 518.24 & 2.19 & ND & ND & ND & ND & ND & ND \\
\hline
\end{tabular}

${ }^{*}$ The relative importance (\%) of each predictor variable is shown in parenthesis.

${ }^{1}$ Akaike's Information Criterion.

${ }^{2} \Delta i$, is the difference between the AIC of a given model and that of the best-ranked model, and quantifies how models compete (best-ranked model: $\Delta i=0$; substantial empirical support: $\Delta i=1-2$; considerable less support: $\Delta i=2-7$; and no support; $\Delta i>10$ ) (Burnham and Anderson, 2002).

${ }^{3}$ Model performance measures: rate of correct predictions of the model (Acc: Accuracy), Area under the ROC curve (AUROC), True Positive rate (TPR). Values are shown as percentages. Measures calculated for models with $\triangle i<2$. ND: Not determined.

PRA: Plant relative abundance; PLC: Plant life cycle; HPS: number of host plant species; RAF: Rainfalls. 
Table A3.3. Logistic models predicting host potyvirus prevalence closely competing with the best-ranked one $\left(\Delta_{i}<2\right)$, and/or the best model with considerable less support $\left(\Delta_{i}>2\right)$.

\begin{tabular}{|c|c|c|c|c|c|c|c|c|}
\hline \multirow[t]{2}{*}{ Model structure* } & \multicolumn{2}{|c|}{$\begin{array}{l}\text { Model } \\
\text { fitting }\end{array}$} & \multicolumn{3}{|c|}{$\begin{array}{l}\text { Model performance } \\
\text { on } 5 \times 2 \mathrm{CV} \text { training set }{ }^{3}\end{array}$} & \multicolumn{3}{|c|}{$\begin{array}{l}\text { Model performance } \\
\text { on validation set }^{3}\end{array}$} \\
\hline & AIC $^{1}$ & $\Delta_{\mathrm{i}}^{2}$ & Acc & AUROC & TPR & Acc & AUROC & TPR \\
\hline \multicolumn{9}{|l|}{ Riparian forests } \\
\hline PRA (67)+RAF (9)+S (7)+SEA (7)+HPD (6)+V(4) & 79.71 & 0.85 & 75 & 91 & 75 & 71 & 84 & 70 \\
\hline PRA (70)+RAF (10)+S (7)+SEA (7)+HPD (6) & 82.89 & 3.18 & ND & ND & ND & ND & ND & ND \\
\hline PRA (77)+S (8)+HPD (7)+SEA (6) & 91.75 & 12.04 & ND & ND & ND & ND & ND & ND \\
\hline PRA (84)+S (9)+HPD (7) & 93.25 & 13.54 & ND & ND & ND & ND & ND & ND \\
\hline PRA $(90)+S(10)$ & 95.62 & 15.91 & ND & ND & ND & ND & ND & ND \\
\hline PARA (100) & 98.73 & 19.02 & ND & ND & ND & ND & ND & ND \\
\hline \multicolumn{9}{|l|}{ Evergreen oak forests } \\
\hline HPS (32)+PRA (26)+HPD (25)+RAF(17) & 20.06 & 0.00 & 94 & 99 & 93 & 70 & 83 & 69 \\
\hline HPS (39)+PRA (31)+HPD (30) & 28.76 & 8.70 & ND & ND & ND & ND & ND & ND \\
\hline PRA (51)+HPD (49) & 37.31 & 17.25 & ND & ND & ND & ND & ND & ND \\
\hline HPD (100) & 39.00 & 18.94 & ND & ND & ND & ND & ND & ND \\
\hline
\end{tabular}

${ }^{*}$ The relative importance (\%) of each predictor variable is shown in parenthesis.

${ }^{1}$ Akaike's Information Criterion.

${ }^{2} \Delta i$, is the difference between the AIC of a given model and that of the best-ranked model, and quantifies how models compete (best-ranked model: $\Delta i=0$; substantial empirical support: $\Delta i=1-2$; considerable less support: $\Delta i=2-7$; and no support; $\Delta i>10$ ) (Burnham and Anderson, 2002).

${ }^{3}$ Model performance measures: rate of correct predictions of the model (Acc: Accuracy), Area under the ROC curve (AUROC), True Positive rate (TPR). Values are shown as percentages. Measures calculated for models with $\Delta i<2$.

ND: Not determined.

PRA: Plant relative abundance; RAF: Rainfalls; S: Species richness; SEA: Season; HPD: Host plant density; V: Plant biomass; HPS: number of host plant species. 
Figure A3.1. Raw J48 Decision Tree of potyvirus prevalence in riparian forests excluding plant identity. In parenthesis: The first number is the total number of instances reaching the leaf. The second number is the number of those instances that are misclassified. PRA: Plant relative abundance; PLC: Plant life cycle; HPS: number of host plant species; RAF: Rainfalls; S: Species richness; SEA: Season; HPD: Host plant density; V: Plant biomass; HPS: number of host plant species.

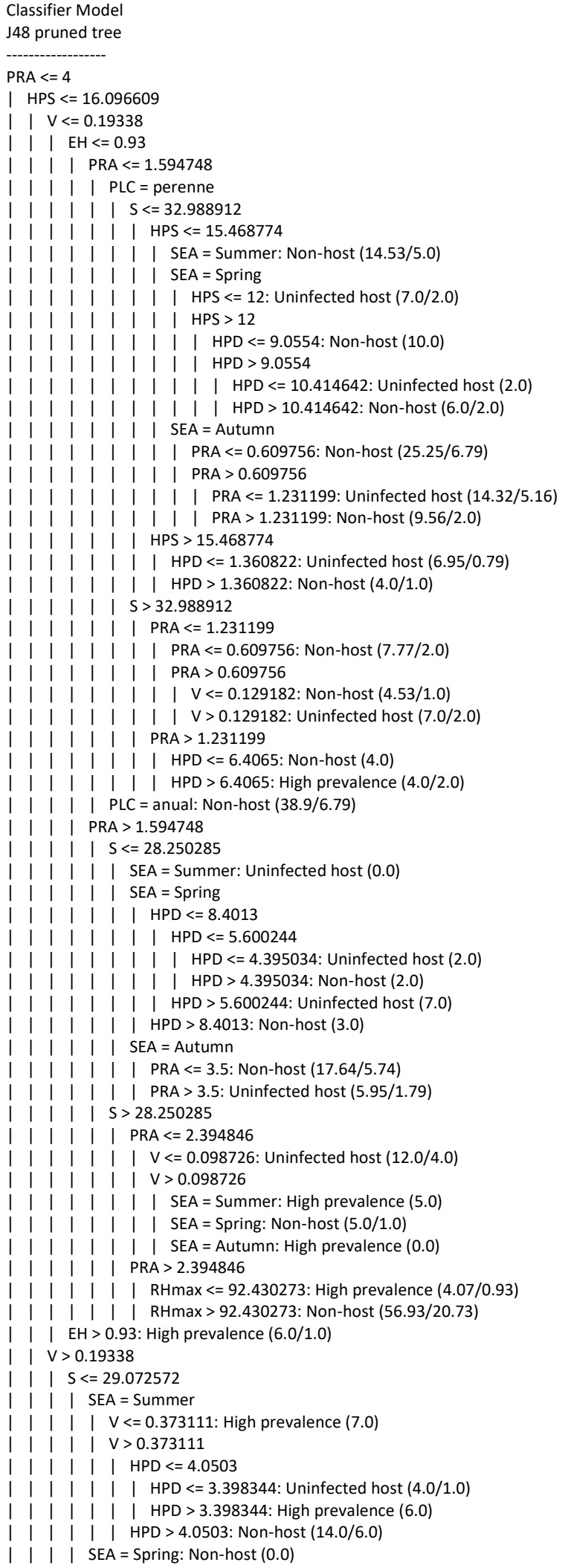


| | | | SEA = Autumn: Non-host (8.6/4.19)

| | | S > 29.072572: High prevalence (38.0)

| HPS $>16.096609$

| | HPS $<=19.970434$

| | | PRA <= 2.85216: High prevalence (30.0)

| | PRA $>2.85216$

| | | | Tmed <= 158.663189: Medium prevalence (12.0)

| | | | Tmed $>158.663189$

| | | | $T \min <=43.754301$

| | | | | RHmax<=96.126623: High prevalence (3.5/0.5)

｜｜｜｜｜RHmax >96.126623: Medium prevalence (3.5)

| | | | | Tmin > 43.754301: High prevalence (10.0)

| HPS $>19.970434$

| | | PRA <=2.049082: Non-host (21.0/8.0)

I | | PRA $>2.049082$

| | | PRA <= 2.728369: High prevalence (7.0/1.0)

| | | | PRA > 2.728369: Medium prevalence (23.0/5.0)

PRA $>4$

| $\mathrm{HPS}<=16.096609$

| | HPD $<=5.3624$

| | | PLC = perenne

| | | tRainfalls $<=430.505004$

| | | | $\mathrm{pH}<=5.004543$

| | | | | | PRA <=5.246646: Non-host (3.0/1.0)

L | | | | | PRA $>5.246646$

| | | | | | | RHmax $<=97.481608$

| | | | | | | | HPD <=3.415964: Non-host (2.0)

| | | | | | | | HPD > 3.415964: Uninfected host (3.0)

| | | | | | | RHmax >97.481608

| | | | | | SEA=Summer: Uninfected host (0.0)

$1|1| 1 \mid$ | | | SEA = Spring

| | | | | | | HPD $<=3.0541$ : Non-host (2.0/1.0)

| | | | | | | | HPD > 3.0541: Uninfected host (3.0)

1 | | | | | | | SEA = Autumn

| | | | | | | HPD $<=3.225308$ : Non-host (2.0/1.0)

| | | | | | | HPD > 3.225308: Low prevalence (3.0/1.0)

| | | | | pH $>5.004543$

| | | | | PRA $<=5.672662$

| | | | | | $\mathrm{S}<=32.459422$

| | | | | | | HPD $<=4.686559$

l | | | | | | | PRA $<=4.905576$ : High prevalence (11.0/2.0)

| | | | | | | | | PRA $>4.905576$

| | | | | | | | | PRA <=5.487805: Uninfected host (2.0/1.0)

l | | | | | | | | | PRA > 5.487805: Non-host (2.9/1.0)

1|||||||| HPD > 4.686559: Low prevalence (7.0/1.0)

| | | | | | S $>32.459422$

| | | | | | $S<=35.955022$ : Medium prevalence (6.0)

| | | | | | | | S>35.955022: Low prevalence (2.0)

| | | | | | PRA $>5.672662$

| | | | | | HPS <= 10.820632: Uninfected host (2.9/0.9)

l | | | | | | HPS $>10.820632$

| | | | | | | $\mathrm{S}<=35.001138$ : Low prevalence (112.9/8.0)

| | | | | | | S $>35.001138$

| | | | | | | | | RHmin <=17.011769: Medium prevalence (3.0)

｜｜｜｜｜｜｜RHmin>17.011769: Low prevalence (2.0)

I | | RAF $>430.505004$

| | | | Tmed $<=150.728161$

| | | | | | HPD $<=3.5099$

| | | | | | $\mathrm{S}<=31$ : Uninfected host (11.9/3.9)

| | | | | | | S>31: Low prevalence (4.0/1.0)

| | | | | HPD > 3.5099: Non-host (11.9/3.0)

| | | | Tmed > 150.728161: High prevalence (3.0)

| | PLC = anual

| | | PRA <= 4.905576: High prevalence (3.0/1.0)

| | | | PRA > 4.905576: Non-host (18.48/3.1)

| $\mid$ HPD $>5.3624$

| | | $\mathrm{S}<=32.007819$

| | | | $\mathrm{EH}<=0.934789$

| | | | | HPD $<=5.920273$

| | | | | PRA <=5.5: Non-host (5.0)

| | | | | | PRA > 5.5: Medium prevalence (2.0)

| | | | | HPD > 5.920273

| | | | | | HPD $<=6.8278$

| | | | | | PRA $<=5.795902$

| | | | | | | | $\mathrm{S}<=29.495309$ : Uninfected host (3.0/1.0)

| | | | | | | S > 29.495309: Medium prevalence (2.0)

| | | | | PRA > 5.795902: Uninfected host (3.0)

1 | | | | | HPD > 6.8278: Non-host (5.0/2.0)

| | | | EH >0.934789: Uninfected host (2.0/1.0)

| | | S > 32.007819: Medium prevalence (6.0)

| HPS $>16.096609$ 


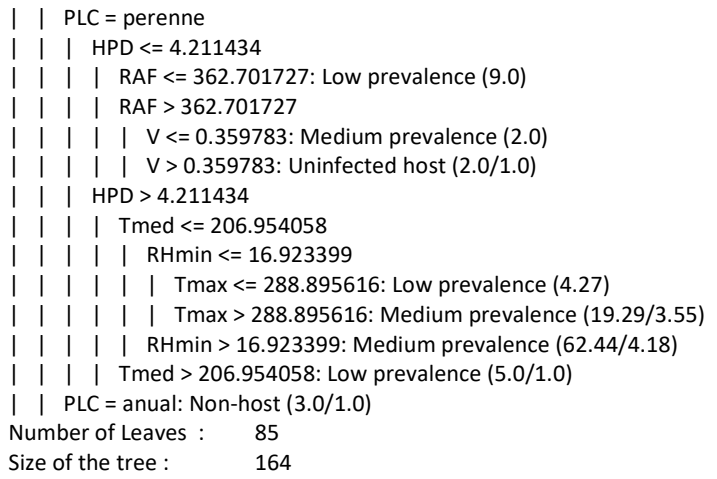


Figure A3.2. Raw J48 Decision Tree of potyvirus prevalence in evergreen oak forests excluding plant identity. In parenthesis: The first number is the total number of instances reaching the leaf. The second number is the number of those instances that are misclassified. PRA: Plant relative abundance; PLC: Plant life cycle; HPS: number of host plant species; RAF: Rainfalls; S: Species richness; SEA: Season; HPD: Host plant density; V: Plant biomass; HPS: number of host plant species.

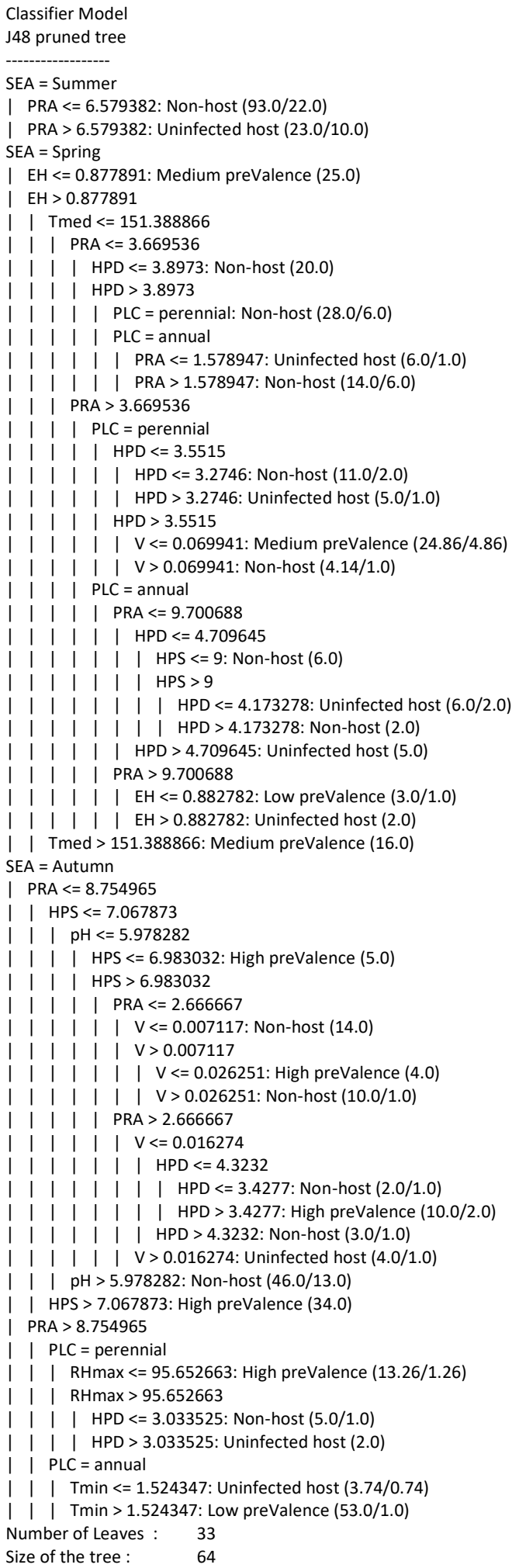




\section{APPENDIX 4}

Analysis of species-specific potyvirus prevalence in wild ecosystems in the Iberian Peninsula 
Table A4.1. Prevalence of CIYVV in host plants analyzed in locations monitored in this study.

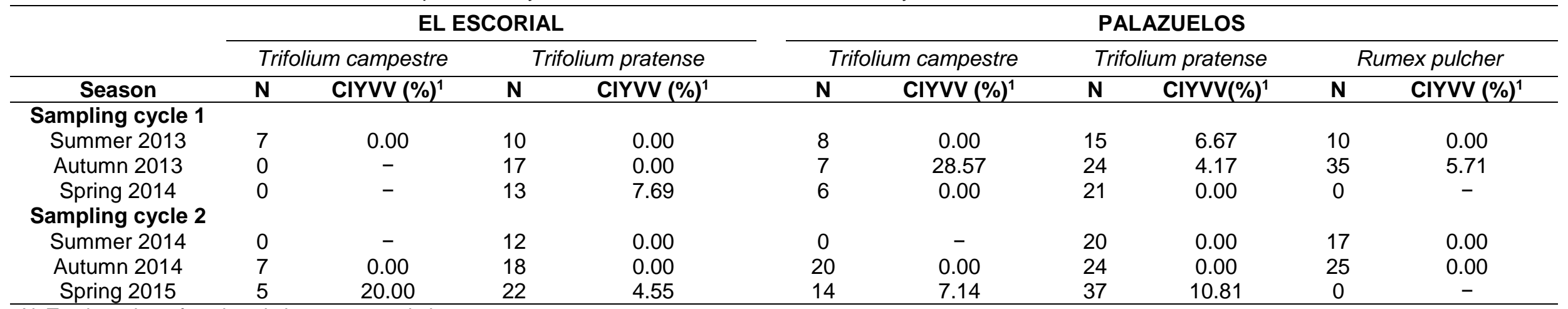

$\mathrm{N}$ : Total number of analysed plants per population.

${ }_{1}^{1}$ Prevalence calculated as percentage of CIYVV-infected plants relative to the total number of plants analysed from a given host species. 
Table A4.2. Prevalence of ENMV in host plants analyzed in locations monitored in this study.

\begin{tabular}{ccc}
\hline & \multicolumn{2}{c}{$\begin{array}{c}\text { Santa Cruz de Retamar } \\
\text { Andryala ragusina }\end{array}$} \\
\hline Season & N & ENMV (\%) \\
\hline Sampling cycle 1 & & 11.50 \\
Summer 2013 & 8 & - \\
Autumn 2013 & 0 & - \\
Spring 2014 & 0 & - \\
Sampling cycle 2 & & - \\
Summer 2014 & 0 & 10.00 \\
Autumn 2014 & 0 & \\
Spring 2015 & 10 &
\end{tabular}

$\mathrm{N}$ : Total number of analysed plants per population.

${ }^{1}$ Prevalence calculated as percentage of ENMV-infected plants relative to the total number of plants analysed from a given host species. 


\section{APPENDIX 5}

Analysis of potyvirus genus-wide genetic diversity in wild ecosystems in the Iberian Peninsula 
Table A5.1. Model selection analyses for potyvirus population haplotype diversity $\left(H_{d}\right)$, genetic diversity $(\pi)$, number of synonymous $\left(d_{S}\right)$ and non-synonymous $\left(d_{N}\right)$ substitutions per site, and selection pressures $\left(d_{N} / d_{S}\right)$. Model structures included host plant density, host relative abundance, plant species richness and plant biomass; and temperature, relative humidity and rainfall in the sampled locations (minimal, maximal and average values) as predictors, and season as covariate. Potyvirus prevalence was also included as predictor of virus evolution parameters. Best-ranked models are shown $(\Delta \mathrm{i}<2)$.

\begin{tabular}{|c|c|c|c|c|c|}
\hline Model structure ${ }^{\star}$ & $r^{\dagger}$ & logLik & AIC $\ddagger$ & $\Delta_{\mathrm{i}}^{\S}$ & $\omega_{i}{ }^{\pi}$ \\
\hline \multicolumn{6}{|l|}{$H_{d}$} \\
\hline$P R A(41)+V(29)+H P D(11)+T_{\text {mean }}(10)+R H_{\text {mean }}(9)$ & $0.94^{*}$ & 33.44 & -52.89 & 0 & 0.32 \\
\hline$P R A(47)+V(35)+T_{\text {mean }}(10)+\mathrm{RH}_{\text {mean }}(9)$ & $0.93^{*}$ & 32.06 & -52.13 & 0.76 & 0.22 \\
\hline $\mathrm{PRA}(40)+\mathrm{V}(28)+\mathrm{HPD}(11)+\mathrm{T}_{\text {mean }}(9)+\mathrm{RH}_{\text {mean }}(8)+\mathrm{RFA}(3)$ & $0.95^{\star}$ & 34.01 & -52.03 & 0.86 & 0.21 \\
\hline$P R A(34)+V(30)+H P D(10)+T_{\text {mean }}(10)+R H_{\text {mean }}(9)+P R E(7)$ & $0.95^{*}$ & 34.01 & -52.03 & 1.88 & 0.13 \\
\hline$P R A(42)+V(26)+H P D(10)+T_{\text {mean }}(10)+\mathrm{RH}_{\text {mean }}(9)+\mathrm{S}(4)$ & $0.95^{*}$ & 33.50 & -51.01 & 1.93 & 0.12 \\
\hline \multicolumn{6}{|l|}{$\pi$} \\
\hline $\mathrm{RAF}(36)+\mathrm{PRA}(32)+\mathrm{S}(16)+\mathrm{RH}$ mean (12)+ $\mathrm{T}_{\text {mean }}(4)$ & $0.85^{\star}$ & 24.46 & -34.91 & 0.00 & 0.33 \\
\hline $\operatorname{PRE}(36)+\mathrm{RAF}(23)+\mathrm{PRA}(18)+\mathrm{S}(12)+\mathrm{R} \mathrm{H}_{\text {mean }}(7)+\mathrm{T}_{\text {mean }}(4)$ & $0.87^{\star}$ & 25.11 & -34.23 & 0.68 & 0.24 \\
\hline $\mathrm{RAF}(33)+\mathrm{PRA}(29)+\mathrm{S}(18)+\mathrm{RH}$ mean (11)+ HPD (5)+ $\mathrm{T}_{\text {mean }}$ (4) & $0.86^{*}$ & 24.87 & -33.74 & 1.17 & 0.18 \\
\hline $\mathrm{RAF}(38)+\mathrm{PRA}(26)+\mathrm{S}(14)+\mathrm{RH}$ mean $(10)+\mathrm{V}(8)+\mathrm{T}_{\text {mean }}(4)$ & $0.85^{*}$ & 24.49 & -32.98 & 1.93 & 0.13 \\
\hline PRE (46)+ RFA (32)+ V (15)+ HPD (7) & $0.80^{*}$ & 22.46 & -32.93 & 1.98 & 0.12 \\
\hline \multicolumn{6}{|l|}{$d_{N}$} \\
\hline$R A F(44)+P R A(30)+R H_{\text {mean }}(11)+S(10)+T_{\text {mean }}(5)$ & $0.87^{\star}$ & 27.52 & -41.04 & 0.00 & 0.16 \\
\hline $\mathrm{RAF}(40)+\mathrm{PRA}(26)+\mathrm{S}(11)+\mathrm{RH}_{\text {mean }}(10)+\mathrm{HPD}(8)+\mathrm{T}_{\text {mean }}(5)$ & $0.88^{*}$ & 28.52 & -41.03 & 0.01 & 0.16 \\
\hline$P R E(36)+R A F(28)+P R A(17)+S(7)+T_{\text {mean }}(6)+\mathrm{RH}_{\text {mean }}(6)$ & $0.88^{*}$ & 28.51 & -41.02 & 0.02 & 0.16 \\
\hline PRE (34)+ RAF (26)+ PRA (15)+ S (8)+ RHmean (6)+ HPD (6)+ Tmean (5) & $0.90^{*}$ & 29.37 & -40.74 & 0.30 & 0.14 \\
\hline$P R E(41)+R A F(31)+P R A(18)+T_{\text {mean }}(5)+R H_{\text {mean }}(5)$ & $0.86^{*}$ & 27.04 & -40.08 & 0.96 & 0.10 \\
\hline$R A F(43)+P R A(28)+R H_{\text {mean }}(11)+S(9)+T_{\text {mean }}(5)+V(4)$ & $0.87^{*}$ & 27.87 & -39.73 & 1.31 & 0.08 \\
\hline$P R E(35)+R A F(28)+P R A(16)+S(7)+T_{\text {mean }}(6)+R H_{\text {mean }}(6)+V(2)$ & $0.89^{*}$ & 28.69 & -39.38 & 1.66 & 0.07 \\
\hline$R A F(51)+P R A(35)+R H_{\text {mean }}(10)+T_{\text {mean }}(4)$ & $0.82^{*}$ & 25.61 & -39.23 & 1.81 & 0.07 \\
\hline $\mathrm{RAF}(41)+\mathrm{PRA}(22)+\mathrm{S}(10)+\mathrm{RH}_{\text {mean }}(9)+\mathrm{HPD}(9)+\mathrm{T}_{\text {mean }}(5)+\mathrm{V}(4)$ & $0.89^{\star}$ & 28.53 & -39.05 & 1.99 & 0.06 \\
\hline \multicolumn{6}{|l|}{$d s$} \\
\hline $\mathrm{PRA}(45)+\mathrm{RAF}(25)+\mathrm{S}(9)+\mathrm{HPD}(8)+\mathrm{RH}_{\text {mean }}(8)+\mathrm{T}_{\text {mean }}(5)$ & $0.93^{*}$ & 15.54 & -15.08 & 0.00 & 0.16 \\
\hline$P R E(32)+P R A(29)+R A F(17)+S(6)+T_{\text {mean }}(6)+\mathrm{RH}_{\text {mean }}(5)+\mathrm{HPD}$ (5) & $0.94^{*}$ & 16.51 & -15.02 & 0.07 & 0.15 \\
\hline$P R E(35)+P R A(31)+R A F(18)+R H_{\text {mean }}(6)+S(5)+T_{\text {mean }}(5)$ & $0.93^{*}$ & 15.44 & -14.89 & 0.19 & 0.14 \\
\hline $\mathrm{PRA}(51)+\mathrm{RAF}(28)+\mathrm{RH}_{\text {mean }}(9)+\mathrm{S}(8)+\mathrm{T}_{\text {mean }}(5)$ & $0.92^{*}$ & 14.35 & -14.69 & 0.39 & 0.13 \\
\hline$P R A(46)+R A F(28)+R H_{\text {mean }}(9)+S(7)+T_{\text {mean }}(5)+V(5)$ & $0.93^{*}$ & 15.22 & -14.44 & 0.64 & 0.11 \\
\hline$P R E(33)+P R A(29)+R A F(18)+T_{\text {mean }}(6)+R H_{\text {mean }}(6)+S(5)+V(3)$ & $0.94^{*}$ & 16.08 & -14.16 & 0.93 & 0.10 \\
\hline $\mathrm{PRA}(38)+\mathrm{RAF}(26)+\mathrm{HPD}(9)+\mathrm{S}(8)+\mathrm{RH}_{\text {mean }}(8)+\mathrm{V}(6)+\mathrm{T}_{\text {mean }}(5)$ & $0.93^{*}$ & 15.75 & -13.50 & 1.58 & 0.07 \\
\hline PRE (38)+ PRA (32)+ RAF (19)+ $T_{\text {mean }}(5)+\mathrm{RH}_{\text {mean }}(5)$ & $0.91^{*}$ & 13.71 & -13.41 & 1.67 & 0.07 \\
\hline $\mathrm{PRE}(30)+\mathrm{PRA}(26)+\mathrm{RAF}(17)+\mathrm{HPD}(6)+\mathrm{S}(6)+\mathrm{T}_{\text {mean }}(6)+\mathrm{RH}$ mean $(5)+\mathrm{V}(4)$ & $0.94^{*}$ & 16.62 & -13.25 & 1.84 & 0.06 \\
\hline
\end{tabular}

"The relative importance (\%) of each predictor variable is shown in parenthesis.

${ }^{\dagger}$ Correlation coefficient. Asterisks indicate significant correlations $(P<0.05)$.

¥Akaike's Information Criterion.

${ }^{\$} \Delta_{i}$, is the difference between the AIC of a given model and that of the best-ranked model, and quantifies how models compete (best-ranked model: $\Delta_{i}=0$; substantial empirical support: $\Delta_{i}=1-2$; considerable less support: $\Delta_{i=2-7}$; and no support; $\Delta>10)$ (Burnham and Anderson, 2002).

"AIC model weight as $\omega_{i}=\exp \left(-0.5 \Delta_{i}\right) / \Sigma \exp \left(-0.5 \Delta_{i}\right)$. The larger the $\omega$, the greater the likelihood of the model relatively to the competing models. Maximum $\omega,=1$.

PRA: Plant relative abundance; PRE: WHMV prevalence; RAF: Rainfalls; RHmean: Average relative humidity; Tmean: Average temperature; RHmean: Average relative humidity; V: Plant biomass; HPD: Host plant density; S: Species richness. 
Table A5.1. Continued.

\begin{tabular}{|c|c|c|c|c|c|}
\hline Model structure* & $r^{\dagger}$ & logLik & $\mathrm{AlC}^{\ddagger}$ & $\Delta_{i}^{\S}$ & $\omega_{i}^{\mathbb{I}}$ \\
\hline \multicolumn{6}{|l|}{$d_{N} / d_{s}$} \\
\hline PRE (49)+ RAF (39)+ V (12) & $0.72^{*}$ & 17.85 & -25.69 & 0.00 & 0.10 \\
\hline $\mathrm{RAF}(44)+\mathrm{S}(20)+\mathrm{PRA}(19)+\mathrm{RH}_{\text {mean }}(13)+\mathrm{T}_{\text {mean }}(4)$ & $0.79^{*}$ & 19.64 & -25.28 & 0.41 & 0.08 \\
\hline $\operatorname{PRE}(44)+\operatorname{RAF}(37)+\mathrm{V}(14)+\mathrm{HPD}(5)$ & $0.74^{*}$ & 18.50 & -25.69 & 0.69 & 0.07 \\
\hline $\operatorname{PRE}(64)+\operatorname{RAF}(36)$ & $0.65^{*}$ & 16.40 & -24.80 & 0.89 & 0.07 \\
\hline$P R E(44)+V(12)+T_{\text {mean }}(3)+R H_{\text {mean }}(4)+R A F(37)$ & $0.78^{*}$ & 19.38 & -24.75 & 0.94 & 0.06 \\
\hline$P R E(34)+H P D(5)+V(10)+S(13)+T_{\text {mean }}(3)+R H_{\text {mean }}(5)+R A F(30)$ & 0.83 & 21.27 & -24.53 & 1.16 & 0.06 \\
\hline $\operatorname{PRE}(39)+\mathrm{HPD}(5)+\mathrm{V}(14)+\mathrm{T}_{\text {mean }}(3)+\mathrm{RH}_{\text {mean }}(3)+\mathrm{RAF}(36)$ & 0.80 & 20.18 & -24.36 & 1.34 & 0.05 \\
\hline $\operatorname{PRE}(54)+S(17)+\operatorname{RAF}(29)$ & $0.69^{*}$ & 17.14 & -24.29 & 1.41 & 0.05 \\
\hline$P R E(35)+P R A(9)+S(15)+T_{\text {mean }}(4)+R H_{\text {mean }}(8)+R A F(29)$ & 0.80 & 20.14 & -24.28 & 1.41 & 0.05 \\
\hline$P R E(46)+V(12)+R H_{\text {mean }}(2)+R A F(39)$ & $0.73^{*}$ & 18.13 & -24.26 & 1.44 & 0.05 \\
\hline $\mathrm{PRE}(39)+\mathrm{V}(8)+\mathrm{S}(13)+\mathrm{Tmean}(4)+\mathrm{RH}_{\text {mean }}(5)+\mathrm{RAF}(32)$ & 0.80 & 20.12 & -24.23 & 1.46 & 0.05 \\
\hline$P R E(45)+S(17)+T_{\text {mean }}(4)+R H_{\text {mean }}(5)+R A F(29)$ & $0.77^{*}$ & 19.06 & -24.13 & 1.57 & 0.05 \\
\hline $\operatorname{PRE}(46)+V(9)+S(13)+\operatorname{RAF}(33)$ & $0.72^{*}$ & 17.97 & -23.95 & 1.75 & 0.04 \\
\hline $\operatorname{PRE}(46)+\operatorname{PRA}(7)+\mathrm{V}(11)+\operatorname{RAF}(37)$ & $0.72^{*}$ & 17.96 & -23.93 & 1.76 & 0.04 \\
\hline $\operatorname{PRE}(40)+\mathrm{HPD}(5)+\mathrm{PRA}(6)+\mathrm{V}(13)+\mathrm{RAF}(36)$ & $0.76^{*}$ & 18.91 & -23.81 & 1.88 & 0.04 \\
\hline $\mathrm{HPD}(5)+\mathrm{PRA}(16)+\mathrm{S}(22)+\mathrm{T}_{\text {mean }}(4)+\mathrm{RH}_{\text {mean }}(13)+\mathrm{RAF}(41)$ & 0.79 & 19.91 & -23.81 & 1.88 & 0.04 \\
\hline $\operatorname{PRE}(49)+\mathrm{V}(12)+T_{\text {mean }}(1)+\operatorname{RAF}(37)$ & $0.72^{*}$ & 17.88 & -23.75 & 1.94 & 0.04 \\
\hline PRE (41)+ HPD (5) + V (14)+ $\mathrm{RH}_{\text {mean }}(2)+\mathrm{RAF}(38)$ & $0.76^{*}$ & 18.87 & -23.73 & 1.96 & 0.04 \\
\hline \multicolumn{6}{|c|}{ 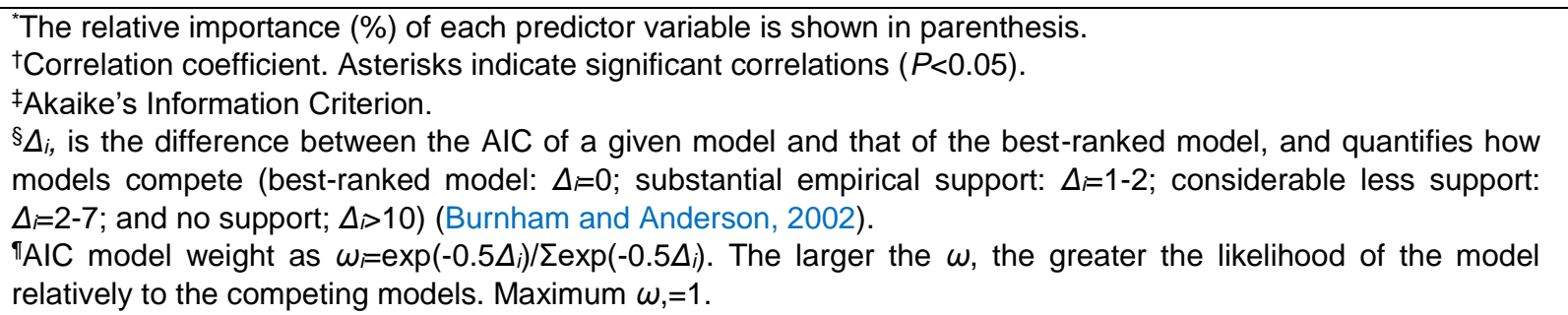 } \\
\hline
\end{tabular}




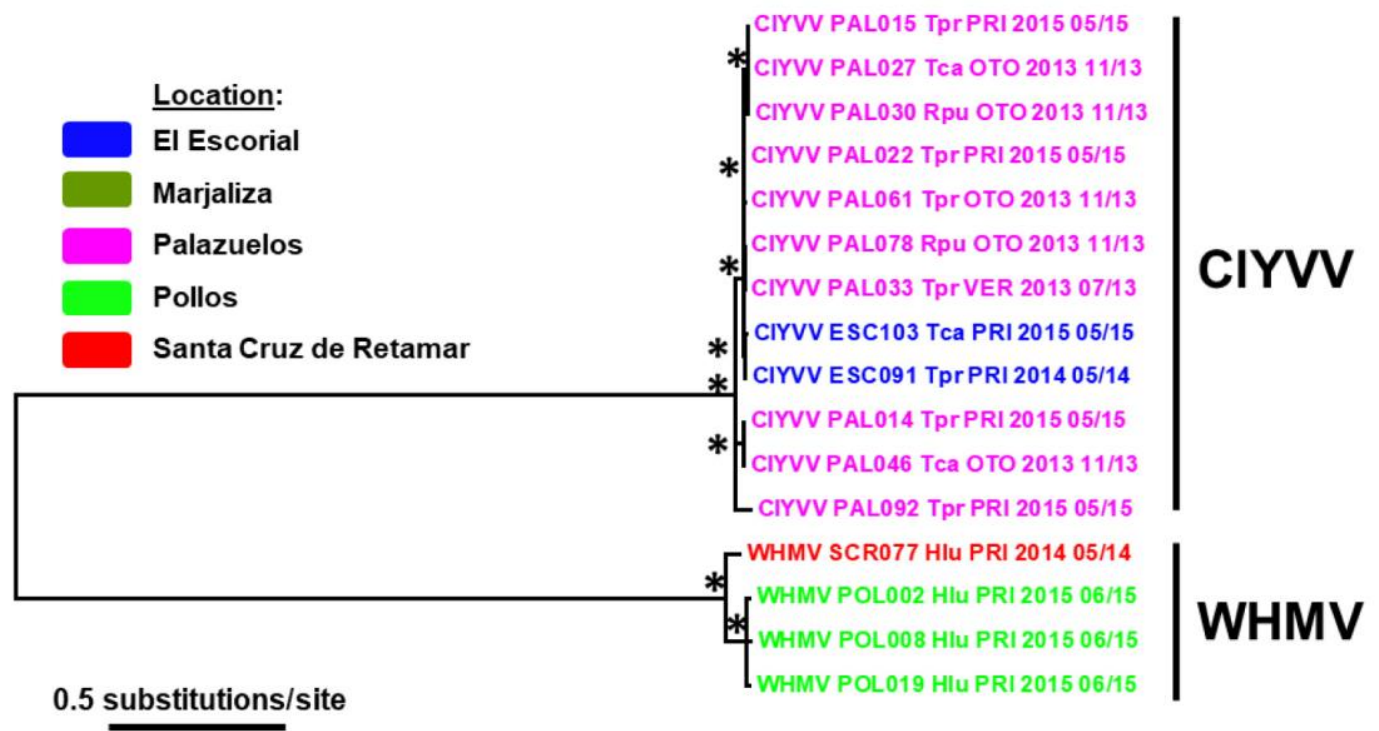

Figure A5.1. Maximum-likelihood phylogeny based on the CP region representing the association between potyvirus phylogeny and geographical location. Asterisks indicates nodes with a bootstrap support $\geq 0.90$.

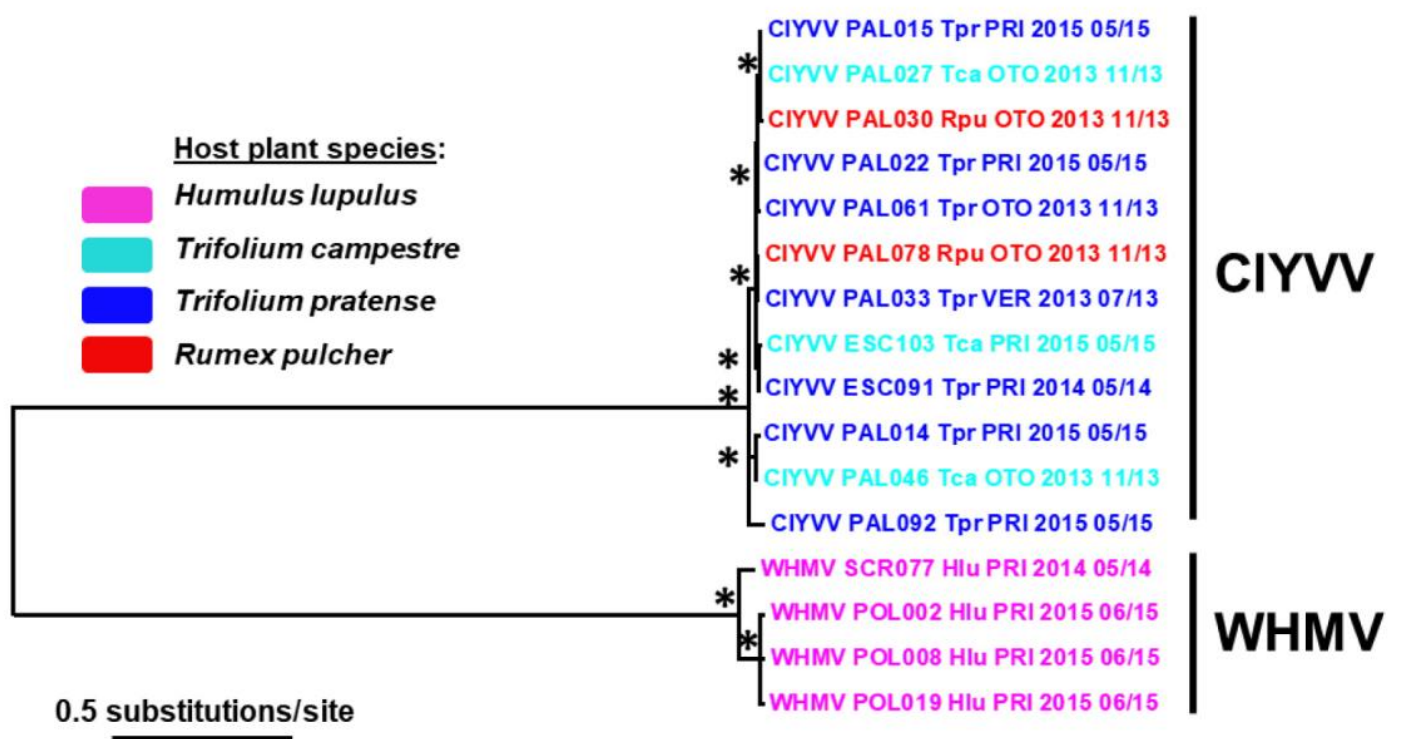

Figure A5.2. Maximum-likelihood phylogeny based on the CP region representing the association between potyvirus phylogeny and host plant species. Asterisks indicates nodes with a bootstrap support $\geq 0.90$. 


\section{APPENDIX 6}

Analysis of potyvirus species-specific genetic diversity in wild ecosystems in the Iberian Peninsula 
Table 4.37. Model selection analyses for WHMV population haplotype diversity $\left(H_{d}\right)$, genetic diversity $(\pi)$, number of synonymous $\left(d_{S}\right)$ and non-synonymous $\left(d_{N}\right)$ substitutions per site, and selection pressures $\left(d_{N} / d_{S}\right)$. Model structures included host plant density, host relative abundance, plant species richness and plant biomass; and temperature, relative humidity and rainfall in the sampled locations (minimal, maximal and average values) as predictors, and season as covariate. WHMV prevalence was also included as predictor of virus evolution parameters. Best-ranked models are shown $(\Delta \mathrm{i}<2)$.

\begin{tabular}{|c|c|c|c|c|c|}
\hline Model structure & $r^{\dagger}$ & logLik & AIC $\ddagger$ & $\Delta_{\mathrm{i}}^{\S}$ & $\omega_{i}^{\pi}$ \\
\hline \multicolumn{6}{|l|}{$H_{d}$} \\
\hline$P R A(30)+R A F(26)+P R E(12)+R H_{\text {mean }}(12)+T_{\text {mean }}(11)+V(9)$ & $0.78^{*}$ & 37.97 & -59.94 & 0.00 & 0.55 \\
\hline$P R A(29)+R A F(26)+P R E(12)+R H_{\text {mean }}(12)+T_{\text {mean }}(11)+V(8)+S(2)$ & $0.79^{*}$ & 38.15 & -58.3 & 1.64 & 0.24 \\
\hline $\mathrm{PRA}(30)+\mathrm{RAF}(26)+\mathrm{RH} \mathrm{H}_{\text {mean }}(12)+\mathrm{PRE}(11)+\mathrm{T}_{\text {mean }}(11)+\mathrm{V}(8)+\mathrm{HPD}$ (2) & $0.78^{*}$ & 37.97 & -57.94 & 2.00 & 0.20 \\
\hline \multicolumn{6}{|l|}{$\pi$} \\
\hline $\mathrm{RAF}(50)+\mathrm{PRE}(27)+\mathrm{R} H_{\text {mean }}(17)+\mathrm{T}_{\text {mean }}(6)$ & $0.71^{*}$ & 66.22 & -120.44 & 0.00 & 0.25 \\
\hline $\mathrm{RAF}(50)+\mathrm{PRE}(28)+\mathrm{RH} \mathrm{Hean}_{\mathrm{n}}(22)$ & $0.67^{*}$ & 65.16 & -120.33 & 0.11 & 0.24 \\
\hline $\mathrm{RAF}(46)+\mathrm{PRE}(28)+\mathrm{RH}_{\text {mean }}(16)+\mathrm{PRA}(4)+\mathrm{T}_{\text {mean }}(6)$ & $0.71^{*}$ & 66.47 & -118.94 & 1.50 & 0.12 \\
\hline $\mathrm{RAF}(45)+\mathrm{PRE}(24)+\mathrm{RHmean}(23)+\mathrm{S}(8)$ & $0.68^{*}$ & 65.37 & -118.74 & 1.70 & 0.11 \\
\hline RAF (50)+PRE (27)+RHmean (17)+Tmean (6)+V (1) & $0.71^{*}$ & 66.32 & -118.64 & 1.80 & 0.10 \\
\hline RAF (49)+PRE (25)+RHmean (17)+Tmean (6)+HPD (2) & $0.71^{*}$ & 66.23 & -118.45 & 1.99 & 0.09 \\
\hline RAF (44)+PRE (23)+RHmean (17)+Tmean (8)+S (9) & $0.71^{*}$ & 66.22 & -118.44 & 2.00 & 0.09 \\
\hline \multicolumn{6}{|l|}{$d_{N}$} \\
\hline $\mathrm{V}(46)+\mathrm{PRE}(21)+\mathrm{RAF}(17)+\mathrm{RH}$ mean $(9)+\mathrm{T}_{\text {mean }}(7)$ & $0.78^{*}$ & 49.73 & -85.46 & 0.00 & 0.38 \\
\hline $\mathrm{V}(41)+\mathrm{PRE}(22)+\mathrm{RAF}(17)+\mathrm{RH}$ mean $(10)+\mathrm{T}_{\text {mean }}(7)+\mathrm{S}(3)$ & $0.80^{*}$ & 50.49 & -84.99 & 0.47 & 0.30 \\
\hline $\mathrm{V}(45)+\mathrm{PRE}(19)+\mathrm{RAF}(16)+\mathrm{RH}$ mean $(10)+\mathrm{T}_{\text {mean }}(7)+\mathrm{PRA}(3)$ & $0.79^{*}$ & 49.97 & -83.94 & 1.52 & 0.18 \\
\hline$V(41)+P R E(18)+R A F(17)+\mathrm{RH}_{\text {mean }}(10)+\mathrm{HPD}(7)+\mathrm{T}_{\text {mean }}(7)$ & $0.78^{*}$ & 49.76 & -83.52 & 1.94 & 0.14 \\
\hline \multicolumn{6}{|l|}{$d s$} \\
\hline $\mathrm{V}(46)+\mathrm{RAF}(13)+\mathrm{PRE}(12)+\mathrm{RH}$ mean $(9)+\mathrm{T}_{\text {mean }}(8)+\mathrm{S}(8)+\mathrm{PRA}(4)$ & $0.81^{*}$ & 43.62 & -69.25 & 0.00 & 0.46 \\
\hline $\mathrm{V}(57)+\mathrm{RAF}(11)+\mathrm{PRE}(10)+\mathrm{RH}$ mean $(9)+\mathrm{T}_{\text {mean }}(8)+\mathrm{PRA}(5)$ & $0.77^{*}$ & 42.35 & -68.7 & 0.55 & 0.35 \\
\hline $\mathrm{V}(44)+\mathrm{RAF}(13)+\mathrm{PRE}(10)+\mathrm{RH}$ mean $(9)+\mathrm{T}_{\text {mean }}(8)+\mathrm{S}(7)+\mathrm{HPD}(5)+\mathrm{PRA}(4)$ & $0.81^{*}$ & 43.79 & -67.57 & 1.67 & 0.20 \\
\hline \multicolumn{6}{|l|}{$d_{N} / d_{s}$} \\
\hline $\mathrm{V}(45)+\mathrm{PRE}(23)+\mathrm{RAF}(16)+\mathrm{RH}$ mean $(9)+\mathrm{T}_{\text {mean }}(7)$ & $0.79^{*}$ & 25.37 & -36.74 & 0.00 & 0.41 \\
\hline $\mathrm{V}(41)+\mathrm{PRE}(23)+\mathrm{RAF}(16)+\mathrm{RH}$ mean $(9)+\mathrm{T}_{\text {mean }}(8)+\mathrm{S}(3)$ & $0.80^{*}$ & 25.98 & -35.96 & 0.78 & 0.28 \\
\hline $\mathrm{V}(43)+\mathrm{PRE}(19)+\mathrm{RAF}(16)+\mathrm{RH}$ mean $(10)+\mathrm{T}_{\text {mean }}(7)+\mathrm{PRA}(5)$ & $0.79^{*}$ & 25.43 & -34.85 & 1.89 & 0.16 \\
\hline $\mathrm{V}(40)+\mathrm{PRE}(20)+\mathrm{RAF}(15)+\mathrm{RH}_{\text {mean }}(9)+\mathrm{HPD}(7)+\mathrm{T}_{\text {mean }}(7)$ & $0.79^{*}$ & 25.39 & -34.77 & 1.96 & 0.15 \\
\hline
\end{tabular}

\footnotetext{
"The relative importance (\%) of each predictor variable is shown in parenthesis.

${ }^{\dagger}$ Correlation coefficient. Asterisks indicate significant correlations $(P<0.05)$.

¥Akaike’s Information Criterion.

${ } \triangle i$, is the difference between the AIC of a given model and that of the best-ranked model, and quantifies how models compete (best-ranked model: $\Delta_{i}=0$; substantial empirical support: $\Delta_{i=1-2}$; considerable less support: $\Delta_{i}=2-7$; and no support; $\Delta>10$ ) (Burnham and Anderson, 2002).

TAIC model weight as $\omega_{i=\exp }\left(-0.5 \Delta_{i}\right) / \Sigma \exp \left(-0.5 \Delta_{i}\right)$. The larger the $\omega$, the greater the likelihood of the model relatively to the competing models. Maximum $\omega,=1$.

PRA: Plant relative abundance; PRE: WHMV prevalence; RAF: Rainfalls; $\mathrm{RH}_{\text {mean: }}$ Average relative humidity; $\mathrm{T}_{\text {mean: }}$ Average temperature; V: Plant biomass; HPD: Host plant density; S: Species richness.
} 